Durchstanzversuche an Flachdecken mit Aussparungen

Franz-Xaver Müller

Aurelio Muttoni

Bruno Thürlimann 


\section{CIP-Kurztitelaufnahme der Deutschen Bibliothek}

Müller, Franz-Xaver:

Durchstanzversuche an Flachdecken mit Aussparungen / von Franz-Xaver Müller; Aurelio Muttoni; Bruno Thürlimann.- Basel; Boston;

Stuttgart: Birkhäuser, 1984.

(Bericht/ Institut für Baustatik und

Konstruktion, ETH Zürich; Nr. 7305-5

ISBN 3-7643-1686-1

NE: Muttoni, Aurelio:; Thürlimann, Bruno:;

Institut für Baustatik und Konstruktion

$<$ Zürich $>$ : Bericht

Nachdruck verboten.

Alle Rechte, insbesondere das der Übersetzung in fremde Sprachen und der Reproduktion auf photostatischem Wege oder durch Mikrofilm, vorbehalten. 


\title{
Durchstanzversuche an Flachdecken mit Aussparungen
}

von

\author{
Dipl. Ing. Franz-Xaver Müller \\ Dipl. Ing. Aurelio Muttoni
}

Prof. Dr. Bruno Thürlimann

Institut für Baustatik und Konstruktion

Eidgenössische Technische Hochschule Zürich 
1. Einleitung

1.1 Problemstellung

1.2 Ziel setzungen

1.3 Versuchsprogramm

1.3.1 Bezeichnung der Versuchskörper

2. Versuchskörper

2.1 Beschreibung

2.1.1 Abmessungen

2.1.2 Schlaffe Biegebewehrung

2.1.3 Vorspannbewehrung

2.1.4 Schubbewehrung

2.1.5 Herstellung und Lagerung

2.1.6 Vorspannung der Platte P 20

2.2 Baustoffe

2.2.1 Bewehrungsstah1

2.2.2 Vorspannstah1

2.2.3 Beton

3. Versuchsdurchführung

3.1 Versuchsanlage

3.1.1 Belastungseinrichtung

3.1.2 Messeinrichtung

3.1.3 Messungen

4. Versuchsresultate

4.1 Verfahren zur Auswertung der Versuchsresul tate

4.1.1 Last

4.1.2 Durchbiegungen

4.1.3 Mittlere Dehnungen

4.1.4 Betonstauchungen

4.1.5 Stahldehnungen der Bügel

4.1.6 Zunahme der Plattendicke

4.1.7 Mittlere Dehnungen in der Aussparung

4.1.8 Bewegungen des Stuitzenfusses

4.1.9 Risse

4.2 A1lgemeines Tragverhalten

4.2.1 Ungerissene Phase

4.2.2 Risslast

4.2.3 Gerissene Phase 
4.3.5 Hauptdehnungen

4.3.6 Stauchungen der Stïtze

4.3.7 Zunahme der Plattendicke

4.3.8 Bruchfiguren 12

4.4 Spezielle Beobachtungen 12

4.4.1 Platte P $16 \quad 12$

4.4.2 Platte $P 17 \quad 13$

4.4.3 Platte P $18 \quad 13$

4.4.4 Platte P $19 \quad 14$

4.4.5 Platte P $20 \quad 14$

$\begin{array}{ll}\text { 4.4.6 Platte P } 22 & 15\end{array}$

$\begin{array}{ll}\text { Zusammenfassung } & 16\end{array}$

$\begin{array}{lr}\text { Résumé } & 17\end{array}$

$\begin{array}{lr}\text { Sommario } & 18\end{array}$

$\begin{array}{lr}\text { Sunmary } & 19\end{array}$

$\begin{array}{lr}\text { Verdankungen } & 20\end{array}$

$\begin{array}{lr}\text { Bezeichnungen } & 21\end{array}$

Literaturverzeichnis $\quad 23$

Bilder 24

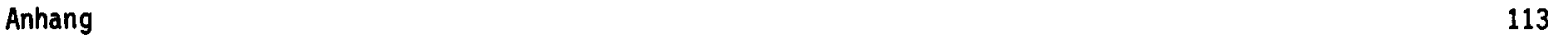




\section{Einleitung}

Im Rahmen des Forschungsprojektes "Vorgespannte Platten" wurden am Institut für Baustatik und Konstruktion der ETH Zürich verschiedene theoretische und experimentelle Arbeiten durchgeführt. Dabei wurden namentlich folgende Probleme behandelt:

- Biegeverhalten von Platten mit Vorspannung ohne Verbund

- Fliessbedingung für Stahlbeton mit Berücksichtigung der Betonzugfestigkeit

- Schubtragverhalten von Stahlbeton- und Spannbetonplatten

- Plastische Berechnung von Stahlbeton

- Durchstanzverhalten von Stahlbeton- und Spannbetonplatten im Bereich von Innenstützen

- Durchstanzverhalten von Stahlbeton- und Spannbetonplatten im Bereich von Rand- und Eckstützen.

Die Resultate dieser Arbeiten sind in den Berichten [1 bis 10] zusammengestellt und veröffentlicht. Zum besseren Verständnis des Tragverhaltens von Flachdecken, die im Bereich von Innenstützen durch Aussparungen geschwächt sind, wurde im Anschiuss an die obgenannten Untersuchungen die im folgenden.beschriebene Versuchsserie geplant und durchgefuihrt.

\section{$1.1 \quad$ Problemstellung}

In einer Flachdecke sind in dem von Querkräften wie auch von Momenten hochbeanspruchten Bereich um die Stützen häufig Aussparungen erforderlich: Leitungskanäle für Heizung, Lüftung, Elektro- und Sanitärinstallationen verlaufen vertikal den Stützen entlang und schwächen diese ohnehin schon kritische Anschlusszone erheblich. Es stellt sich nun einerseits die Frage, um wieviel der Schubtragwiderstand des Stützenanschlusses durch die Anordnung einer Aussparung von bestimmter Grösse und Lage reduziert wird, und andererseits, mit welchen Massnahmen dieser Bauteil verstärkt werden kann, um die durch die Aussparung verursachte Schwächung zu kompensieren.

Der Einfluss von Aussparungen auf das Tragverhalten wurde u.a. in $[11,12,13,14,15]$ untersucht. In einigen Versuchen beschränkte man sich auf wenige Messungen, so dass sich über den inneren Kräfteverlust nur wenig aussagen lässt. In anderen Fällen wurde der Modellmassstab so klein gewählt, dass sich die Resultate nur mit Vorbehalt auf wirkliche Flachdecken übertragen lassen. Zur Verstärkung des Stützenbereichs werden vor allem einbetonierte Stahlelemente verwendet, wie z.B. in [16] berichtet wird. Lösungen mit vertikalen Bügeln werden selten ausgeführt, und es sind keine entsprechenden Versuche bekannt.

\subsection{Zielsetzungen}

Die sechs Versuche sollen beitragen, das Schubtragverhalten von Flachdecken aus Stahl- und Spannbeton mit Aussparungen im Stützenbereich besser zu verstehen und Aufschluss zu geben über die Verformungsfähigkeit sowie den Verlauf der inneren Kräfte.

Folgende Parameter wurden variiert:

- Grösse der Aussparung

- Schubbewehrung

- Vorspannung ohne Verbund.

Die Versuche fanden im Anschluss an die entsprechenden Versuche bei Innenstiutzen statt. Die folgenden Parameter wurden aus diesen Versuchen übernommen und konstant gehalten:

- Abmessungen der Versuchskörper

- Biegebewehrung bzw. Spannglieder

- Materialkennwerte.

Bei allen Platten wurde das Tragverhalten bis zum Bruch beobachtet, wobei die Deformation stufenweise gesteigert wurde. 
Zur Ausführung gelangten fünf schlaff bewehrte und eine vorgespannte Platte (Bild 1). Als Ausgangsversuch für die ersten fünf Platten diente die orthogonal bewehrte Platte P 5 aus der Versuchsserie 'Innenstützen' [6]. Die Grösse und die Lage der Aussparung wurden so gewählt, dass bei genau gleicher Bewehrung wie im Ausgangsversuch in jeder Richtung mindestens zwei Bewehrungsstäbe geschnitten und durch Zulagen ersetzt werden mussten, um nicht durch die Aussparung zu verlaufen. Die Breite der Aussparung in tangentialer Richtung wurde variiert.

Zwei Platten mit Aussparungen wurden mit einer speziellen Schubbewehrung ausgeführt. Da kein direkter Vergleichsversuch mit einer gleichartig verteilten Schubbewehrung an einer Innenstuitze ohne Aussparung vorhanden war, wurde das Programm nachträglich noch um einen solchen Versuch erweitert.

In der vorgespannten Platte wurden die Spannglieder in der gleicher Weise angeordnet wie in der Platte P 8 [6]. Die Aussparungen kamen in die vier Feider neben den Kabelgruppen zu liegen. Im Gegensatz zur Platte P 8 wurde eine orthogonale schlaffe Bewehrung verlegt. Ein Vergleich mit P 8 ist also nur mit Vorbehalt möglich.

\subsubsection{Bezeichnung der Versuchskörper}

Die Numerierung schliesst an die Versuchsserie $P 10$ bis $P 15$ an [8]. Der Versuchskörper $P 21$ fehlt, da $P 22$ vorgezogen und $P 21$ später nicht mehr ausgeführt wurde. 


\section{Versuchskörper}

\section{$2.1 \quad$ Beschreibung}

\subsubsection{Abmessungen}

Die Abmessungen der Platten gehen aus Bild 2 hervor. Bei allen Versuchskörpern wurden die schon bei den Platten $P 2$ bis $P 9$ gewählten Abmessungen beibehalten. Die theoretische Plattenstärke von $180 \mathrm{~mm}$ erlaubte auch im Falle einer Vorspannung eine in der Praxis übliche konstruktive Ausbildung. Sie stellt aber eine untere Grenze dar, unter welcher die in der Praxis vorkommenden Verhältnisse kaum mehr reproduzierbar sind. Die effektiven Plattenstärken wurden nachträglich gemessen und sind Bild 20 zu entnehmen.

\subsubsection{Schlaffe Biegebewehrung}

Die Anordnung der schlaffen Bewehrung geht aus den Bildern 3 bis 8 sowie 11 hervor. Die Biegebewehrung der Platten P 16, P 17, P 18, P 19 und P 22 bestand aus orthogonal verlegten Bewehrungsstäben Durchmesser $16 \mathrm{~mm}$ mit einem gegenseitigen Abstand von $100 \mathrm{~mm}$. Die gegen die Aussparung führenden Stäbe wurden ohne Endhaken bis an deren Rand geführt. Die Randzone der Aussparung wurde in den Platten P 16, P 17 und P 18 mit Steckbügeln $\emptyset 12 \mathrm{~mm}$ eingefasst (Bild 9a). In der Platte $P 19$ wurde jeder gegen die Aussparung führende Bewehrungsstab mit zwei Steckbïgeln $\emptyset 8 \mathrm{~mm}$ versehen (Bild $9 \mathrm{~b}$ ). Für jeden in der Aussparung unterbrochenen Stab wurde ein Stab (Zulage) neben der Aussparung zugelegt. Somit blieb die Stahlfläche und damit auch der Biegewiderstand in einem Schnitt quer durch die Platte und durch die Aussparung erhalten. Die Unterseite wurde durchgehend mit Stäben $\emptyset 8 \mathrm{~mm}$ und einer Maschenweite von ebenfalls $100 \mathrm{~mm}$ bewehrt.

Die mit Spanngliedern versehene Platte P 20 wurde oben mit Stäben $\emptyset 10 \mathrm{~mm}$, Stababstand $100 \mathrm{~mm}$, bewehrt. Die Stäbe wurden am Rand der Aussparung abgebogen und dabei mit der unteren Bewehrung $08 \mathrm{~mm}$ gestossen. Aus konstruktiven Gründen wurden in der $y$-Richtung die obere Bewehrung ganz, die untere halb umgebogen. Dementsprechend wurden in $x$-Richtung die untere Bewehrung ganz und die obere nur halb abgebogen (Bild $9 \mathrm{c}$ ).

In allen Platten wurde eine kräftige Randbewehrung angeordnet.

\subsubsection{Vorspannbewehrung}

Die Platte P 20 wurde vorgespannt. Die Vorspannung war wie in der Platte P 8 [6] in zwei Gruppen zu je sechs Kabeln angeordnet, die sich über der Stütze rechtwinklig kreuzten. Die Kabel verliefen in vertikaler Richtung polygonal und waren exzentrisch verankert (Bilder 10 und 12).

\subsubsection{Schubbewehrung}

Im Rahmen der Forschungsprojektes wurden schon wiederholt Schubbewehrungen angewendet: geschlossene Bügel in der Platte P 6 [6] sowie offene Bügel im Plattenstreifen PS 8 und in der Platte P 3 [4]. In der Platte $P 11$ wurde ein Bügel in der Form eines $U$ verwendet, der oben geschlossen und unten offen war. Die Verankerung erfolgte über Stahlplättchen, die an den beiden Enden montiert waren, und die in die Druckzone zu liegen kamen. Diese Bügel konnten einfach verlegt werden, sie haben sich in den Versuchen gut bewährt.

In den Platten P 17 und P 22 wurde derselbe Bügeltyp wie in der Platte P 11 verwendet. Wie für die Biegebewehrung wurde auch für den Bügel Topar $S \operatorname{tah} 1$ verwendet (siehe Abschnitt 2.2.1). Die Verbindung Bewehrungsstahl - Ankerplatte erfolgte mittels Schweissung. Für die Bügel der Platte $P 22$ wurde ein Loch in die Stahlplatte gebohrt, der Bewehrungsstahl hindurchgesteckt und anschliessend mit einer Kehlnaht verbunden. Die Bügel der Platte $P 17$ wurden von der Losinger AG hergestel1t. Der Bewehrungsstab wurde an ein mit einem Nocken versehenes Stahlplättchen mit Widerstandsschweissung befestigt. Beide Verbindungsarten konnten im Zugversuch die gesamte Stabfliesskraft des Bewehrungsstahls übertragen. Der Bruch erfolgte ausserhalb der Verbindungsstelle (Bild 15b). In der Platte $P 19$ wurde nochmals ein ähnlicher Bügeltyp angewendet wie in der Platte $P$ 17, allerdings mit einem engeren Schenkelabstand. Die verschiedenen Bügel sind im Bild $9 d$ dargestel1t. 
In der Platte $P 22$ wurden in 24 Strahlen je vier Bügel so angeordnet, dass in jedem Schnitt unter $45^{\circ}$ durch einen Strahl immer zwei vertikale Stäbe geschnitten wurden (Bilder 8 und 13c). In jedem Kreisschnitt wurden daher $24 \times 2=48$ Stäbe geschnitten, was bei einer Fliesspannung $f_{\text {sy }}$ von $500 \mathrm{~N} / \mathrm{mm}^{2}$ einer totalen Fliesskraft von $1200 \mathrm{kN}$ entspricht. Die Bügelbewehrung reichte bis zu einem Abstand von $750 \mathrm{~mm}$ vom Zentrum. In der Platte P 17 wurde dieselbe Anordnung gewählt wie in der Platte P 22 (Bilder 4 und 13a). Die Strahlen, die durch die Aussparung führten, wurden dabei tangential verschoben. Es wurde speziell darauf geachtet, dass die Bügel in den Abbiegungen je einen Stab umfassten. Damit sollte auch oben in der Zugzone eine gute Verankerung gewährleistet werden. In der Platte $P 19$ wurde lediglich diejenige Plattenhälfte mit Bügeln bewehrt, in der die Aussparung lag (Bilder 6 und 13b). Das Ziel war, die Schwächung der Aussparung mit den Bïgeln zu kompensieren und dabei mindestens die Traglast von Platte P 5 (keine Bügel, keine Aussparung) zu erreichen.

\subsubsection{Herstellung und Lagerung}

Die Versuchskörper wurden in der Forschungshalle der ETH-Hönggerberg hergestellt. Die Herstellung der Versuchskörper erfolgte in einer Holzschalung. Für jede Platte und die dazugehörenden Betonproben waren insgesamt 1.3 Kubikmeter hochwertiger Beton BH PC $300 \mathrm{~kg} / \mathrm{m}^{3}$ erforderlich, der mit einem Zwangsmischer in fünf bis sechs Mischungen aufbereitet wurde. Der Beton wurde sorgfältig mit einer Hochfrequenz-Fibriernadel $56 \mathrm{~mm}$ und, wo dies nicht möglich war, mit einer solchen $35 \mathrm{~mm}$ verdichtet. Die Betonoberfläche wurde abgerieben und mit einer Plastikfolie abgedeckt. Sechs Tage nach dem Betonieren wurden die Platten ausgeschalt und bei einer Raumtemperatur von ca. $20{ }^{\circ} \mathrm{C}$ gelagert. In der Zeit zwischen Herstellung und Versuchsbeginn wurden weitere Vorbereitungen getroffen wie Aufkleben der Messbolzen und der Dehnmessstreifen sowie Anstreichen der Platte zwecks besserem Erkennen der Risse.

\subsubsection{Vorspannung der Platte $P 20$}

Vor dem Spannvorgang wurde die Platte in die Versuchsanlage eingebaut und anschliessend daran eine Nullmessung durchgeführt. Dann wurde die volle Vorspannung aufgebracht. Die durchschnittliche Vorspannkraft pro Kabel betrug $176 \mathrm{kN}$, was etwa $0.68 \cdot \mathrm{f}_{\mathrm{pt}}$ entspricht. Die Bestimung der Kraft erfolgte mit Hilfe eines Manometers an der Presse und Kraftmessdosen. Acht Kabel waren mit Kraftmessdosen ausgerüstet.

\subsection{Baustoffe}

\subsubsection{Bewehrungsstah1}

In allen Platten mit Ausnahme von Platte P 20 wurde der neue Stahl Topar $500 \mathrm{~S}$ verwendet. Dieser Stahl wird nach dem Walzvorgang mit einem speziellen Verfahren abgeschreckt und anschliessend durch die Kernwärme vergütet. Das Verhalten liegt zwischen demjenigen eines naturharten und eines kaltverformten Stahls. Wie das Bild 15 zeigt, wurde in den Kennwerten verhältnismässig grosse Streuungen festgestellt. Die Festigkeitswerte wurden verformungsgesteuert mit einer servogesteuerten Universalprüfmaschine (Schenck) ermittelt, die Dehnungsgeschwindigkeiten $\dot{\varepsilon}$ betrugen 0.3 und $3.0 \% / \mathrm{min}$. Die statischen Festigkeitswerte wurden nach zweiminutigem Konstanthalten der Dehnungen bestimmt. Die Bestimmung des Elastizitätsmoduls erfolgte mittels Feindehnungsmessung über $100 \mathrm{~mm}$.

\subsubsection{Vorspannstahl}

Als Vorspanngl ieder wurden Stahlton-Cona-Kabel 0.6 " verwendet, bestehend aus einer 7-drahtigen Litze mit einer theoretischen Fläche von $143 \mathrm{~mm}^{2}$. Der Elastizitätsmodul der Litze betrug $E_{p}=198.2 \mathrm{kN} / \mathrm{mm}^{2}$, die Spannung $f_{\text {py }}$ bei $0.2 \%$ bleibender Dehnung betrug $1650 \mathrm{~N} / \mathrm{mm}^{2}[6]$

\subsubsection{Beton}

Die Zusammensetzung des Frischbetons war für alle Platten gleich. Das Grösstkorn betrug $16 \mathrm{~mm}$. Als Bindemitte 1 wurde Portlandzement verwendet mit einer Dosierung von $300 \mathrm{~kg} / \mathrm{m}^{3}$. Der Wasserzementfaktor $1 \mathrm{ag}$ um 0.5 . Zur besseren Verarbeitbarkeit wurden $0.5 \mathrm{~kg}$ Verflüssiger 'Sikament' auf $100 \mathrm{~kg}$ Zement zugegeben. Ueber die 
Zusammensetzung der Zuschlagstoffe und das Mischen des Betons wird in [6] berichtet. Die Druckfestigkeit wurde in Zylinder- und Würfelversuchen, die Zugfestigkeit in direkten Zug-, Brasilianer- und Stempe1versuchen bestimnt. 


\section{Versuchsdurchführung}

\subsection{Versuchsanlage}

Die für die Platten $P 4$ bis $P 9$ verwendete Versuchsanlage wurde mit einigen wenigen Modifikationen übernommen (Bild 16). Der Stützenfuss der Versuchskörpers wurde auf einer Flachpresse allseitig gelenkig gelagert, um eine Verdrehung zu gestatten. Die Stabilisierung erfolgte über vier auf dem Aufspannboden befestigte Sicherungsstangen, in denen die Kraft kontrolliert wurde. Der Stahlrahmen diente allein Sicherungszwecken.

\subsubsection{Belastungseinrichtung}

Die Belastung wurde mit acht 135-kN-Zugkolben aufgebracht, die entlang einem Kreis mit Durchmesser $2600 \mathrm{~mm}$ verteilt waren. Die Kolben waren unten an einem auf dem Aufspannboden befestigten Stahlrahmen gelenkig gelagert und oben mit Zugstangen verbunden, die die Last über Stahlplatten 100/100/15 mm auf den Versuchskörper übertrugen. Der Druck für die Kolben wurde von einer servohydraulisch gesteuerten Pumpe geliefert.

In der Platte $P 20$ waren in einem radialen Abstand von $1200 \mathrm{~mm}$ auf den Hauptachsen vier zusätzliche $135-\mathrm{kN}-$ Zugkolben angebracht. Diese waren an eine separate Handpumpe angeschlossen und erlaubten es, die Aufbringung der Belastung und die Kompensation der Umlenkkräfte zu steuern. In der Platte $P 22$ wurden ab Laststufe 16 vier zusätzliche Zugkolben eingesetzt wie in der Platte $P$ 20. In diesem Fall waren diese aber zu den übrigen acht Zugkolben parallel geschaltet. Diese Anordnung war notwendig, um die erwartete Bruchlast aufbringen zu können.

\subsubsection{Messeinrichtung}

Zur Erfassung der Daten stand eine elektronische Mess- und Datenerfassungsanlage zur Verfügung. Die Messungen wurden vom Computer digitalisiert, in Protokollform ausgedruckt und in einem File abgespeichert. Alle Kraftmessungen, die Randdurchbiegungsmessungen mittels induktiver Wegaufnehmer sowie die Messungen der Betonstauchungen mittels Strain Gauges waren fest verdrahtet und konnten innert Sekunden automatisch abgefragt werden. Die Deformetermessungen, die Durchbiegungsmessungen mit der Messstange sowie die Messung der Plattendicke wurden einzeln abgefragt, wobei das Messgerät für jede einzelne Messung zuerst in die entsprechende Messposition gesetzt werden musste.

Auf einem $x-y$-Schreiber wurden laufend Last und Randdurchbiegung aufgezeichnet. In einigen Versuchen wurde auch die Messung der Dicke an einer ausgewählten Stelle kontinuierlich auf einem Plotter erfasst.

\subsubsection{Messungen}

Das Messprogramm geht aus Bild 17 (automatische Messungen, Durchbiegungen, Plattendicke) sowie den Bildern 18 und 19 (Deformetermessungen) hervor. Die Messungen wurden in der Nähe der Stütze und der Aussparung konzentriert.

Folgende Messungen wurden im einzelnen durchgeführt:

Vertikalkraft:

- Messung der Auflagerreaktion mit einer $2000 \mathrm{kN}$ Flachpresse, an die ein Druckgeber angeschlossen war.

- Messung der Lasten mit $150 \mathrm{kN}$ Kraftmessdosen, die an vier ( $P 20$ und P 22: 6) der acht (12) Zugkolben angebracht waren.

- Messung der Kraft in den vier Stabilisierungsstangen (150 kN Kraftmessdosen).

- Messung des Drucks im oelhydraulischen System.

Vorspannkraft:

- Messung der Kabelkraft an acht von zwölf Spanngliedern mittels 300 kN Kraftmessdosen. 
Vertikalverschiebungen:

- Messung mit vier induktiven Wegaufnehmern

- Messung mit einer versetzbaren Messstange

- Messung der Lagereinsenkung mit mechanischen Messuhren (P 19 und P 20: Deformeter).

Die induktiven Wegaufnehmer waren in einem radialen Abstand von $1300 \mathrm{~mm}$ (75 mm vom Rand) auf den Hauptachsen angeordnet. Mit der Messstange wurden die Durchbiegungsprofile aufgenommen.

Betonstauchung:

- Messung der lokalen radialen und tangentialen Betonstauchung mit Dehnmessstreifen (Strain Gauges), Basislänge $60 \mathrm{~mm}$.

- Messung der vertikalen Betonstauchungen am Stützenkopf bei den Platten P 16 bis P 19 mit Dehnmessstreifen, Basislänge $60 \mathrm{~mm}$.

Stahldehnung der Bügel:

- Messung mittels Dehnmessstreifen, Basislänge $20 \mathrm{~mm}$ (nur in P 17 und P 22).

Mittlere Dehnungen:

- Messung der gegenseitigen Verschiebung der einzelnen Messpunkte mittels Deformeter unterschiedlicher Basislänge: 100, 141, 200, 283 und $400 \mathrm{~mm}$.

Die versetzbaren Dehnungsmessgeräte (Deformeter) wurden auf Bolzen aufgesetzt, die auf der Unterseite (Druckzone) sowie in der Aussparung direkt auf den Beton und auf der Oberseite (Zugzone) auf die obere Bewehrung geklebt waren. Gemessen wurde strahlenförmig, wobei die einzelnen Strahlen zu einem zusarmenhängenden Netz verbunden waren (Bilder 18 und 19).

Zunahme der Plattendicke:

- Messung mittels Dickenmessgerät, in das ein induktiver Wegaufnehmer eingebaut war (Bild 72).

Rissweiten :

- Messung mitteis Rissemassstab

Neigungswinke]:

- Messung am Stuitzenfuss mit Neigungsmesser (nur in $P 19$ und $P 20$ ).

\subsection{Versuchsablauf}

Alle Platten wurden schrittweise bis zum Bruch belastet (Bild 21). Zur Versuchssteuerung waren am Plattenrand auf der y-Achse zwei zusätzliche induktive Wegaufnehmer montiert. Die Randdurchbiegung wurde stufenweise gesteigert, mit einer Geschwindigkeit von ca. $1 \mathrm{~mm} / \mathrm{min}$. Auf dem neuen Niveau wurde zuerst die Last zwei Minuten lang konstant gehalten, und anschliessend wurde eine Ablesung der fest verdrahteten Messstellen vorgenommen (LS n.1). Anschliessend folgten die Messungen mit den versetzbaren Messgeräten (LS $n$ ), was rund eine Stunde in Anspruch nahm. In dieser Phase wurde die Randdurchbiegung w konstant gehalten. Zum Abschluss wurden die fest verdrahteten Messstellen nochmals abgefragt (LS n.2). Aus der Differenz von der ersten zur zweiten Messung kann die Relaxation bestimmt werden. Bevor die Deformation weiter gesteigert wurde, folgte noch eine Phase, in der die Risse gemessen und weitere Beobachtungen durchgeführt wurden. In einigen Laststufen wurde allein die automatische Messung (LS n.1) durchgeführt und dann sofort weitergefahren. Falls der Versuch über Nacht unterbrochen werden musste, wurde bei der Wiederaufnahme eine automatische Messung bei der vorher erreichten Randdurchbiegung durchgeführt und anschliessend weiterbelastet.

Der Versuch P 22 wurde in der eben beschriebenen Weise bis LS 19 durchgeführt. Dann folgte eine Pause von 15 Tagen. Die folgenden Laststufen bis zum Bruch wurden anlässlich einer Demonstration gefahren. Da dabei nur wenig Zeit für Messungen und Beobachtungen zur Verfügung stand, konnten in diesen letzten Laststufen nur einige ausgewählte Messungen durchgeführt werden. 
4. Versuchsresul tate

4.1 Verfahren zur Auswertung der Versuchsresultate

\subsubsection{Last}

Die in den Bildern 23 bis 28 dargestellte, am Stützenfuss gemessene Reaktion $R$ setzt sich aus der über die Zugkolben und die Stabilisierungsstangen eingeleiteten Last und dem Eigengewicht zusammen. Daher entspricht die Reaktion $R$ bei der Durchbiegung $w=0$ dem Eigengewicht sowie den zu diesem Zeitpunkt über die Sicherungsstangen eingeleiteten Kräften. Der Einfachheit halber wird im folgenden die Reaktion $R$ am Stützenfuss mit 'Last' bezeichnet.

Die Bruchlast $R_{u}$ entspricht der höchsten gemessenen Last. In den Versuchen $P 16$ und $P 20$ musste diese Last vom $x-y$-Schreiber abgelesen werden, da direkt vor dem Bruch keine automatische Messung mehr möglich war. Daher sind diese Werte in den Tabellen der Bilder 23 und 27 nicht aufgeführt. Wie im Bild 21 dargestellt, wird die Deformation während einer Laststufe ab der Messung LS n.1 bis zur Messung LS n.2 konstant gehaiten. Infolge Relaxation nimmt die Last $R$ im gleichen Zeitraum ab, von der Anfangslast in LS $n .1$ auf die Endlast in LS n.2. In den Bildern 22, 60 bis 65 und 70 bis 82 sind für $R$ die Endlasten, also die Werte der Messungen LS $n .2$ aufgetragen. Es ist zu beachten, dass demgegenüber die Bruchlast $R_{u}$ einer Anfangslast entspricht.

Der Fehler in der Bestimung der Reaktionskraft $\mathrm{R}$ kann aufgrund der statisch unbestimmten Messanordnung abgeschätzt werden und beträgt höchstens $\pm 2 \mathrm{kN}$.

\subsubsection{Durchbiegungen}

Die in den Bildern 23 bis 28 aufgezeichnete Randdurchbiegung w entspricht dem arithmetischen Mittel aus den beiden mittels induktiver Wegaufnehmer gemessenen Durchbiegungen bei $x=0, y= \pm 1300 \mathrm{~mm}$. Die in den Bildern 31 bis 36 dargestellten radialen Durchbiegungsprofile wurden in den Versuchen $P 16$ bis $P 19$ gemittelt aus den mit der Messstange gemessenen vertikalen Verschiebungen, die bezüglich der $x$-Achse symmetrisch sind. Die Platten P 20 und P 22 wiesen mit der y-Achse eine zweite Symetrieachse auf. Hier wurde zwischen den vier Quadranten gemittelt.

Die Lagereinsenkung, die Rotation des Stützenfusses auf dem Lager sowie die Verkürzung des Stützenstummels, auf dem die Platte gelagert war, wurden berücksichtigt. Die Stiutzenachse wird in den Bildern immer vertikal gehalten. Die Genauigkeit der vertikalen Verschiebungsmessung hängt vor allem von der Messung der vertikalen Verschiebung des Stützenfusses ab (siehe auch Abschnitt 4.1.8). Sie beträgt etwa $\pm 0.1 \mathrm{~mm}$, wobei die Abweichungen nur für kleinere Lasten ins Gewicht fallen.

\subsubsection{Mittlere Dehnungen}

Die mittels Deformeter gemessenen mittleren Dehnungen wurden analog den Durchbiegungen arithmetisch gemittelt und zwar bezüglich der $x$-Achse in den Versuchen $P 16$ bis $P 19$ und bezüglich der $x-$ und der $y$ Achse in den Versuchen $P 20$ und $P$ 22. Die groben Fehler wurden dabei entdeckt und eliminiert. Um die zufälligen Fehler auszugleichen, wurden die gemittel ten Dehnungen in den Stäben eines statisch unbestimmten Ersatzfachwerkes, das dem Messnetz entsprach, als Zwängungsdehnungen eingegeben und daraus die Knotenverschiebungen ermittelt. Aus den Knotenverschiebungen konnten nun beliebige Dehnungen gerechnet werden.

Die Bilder 18 und 19 zeigen das Messnetz und das Auswertungsnetz der PTatten $P 16$ und $P$ 20. Die entsprechenden Netze für die Platten P 17, P 18, P 19 und P 22 unterscheiden sich nur geringfügig von demjenigen der Platte P 16. Die Messebene 'Unterseite' lag um die Dicke der Messbolzen unter der Unterseite der Betondruckzone, die Messebene 'Oberseite' lag um Bolzendicke über der obersten Bewehrungslage. Die genaue Lage der beiden Messebenen kann dem Bild 20 entnommen werden, wo auch die gemessenen Plattenstärken, statischen Höhen und Betonüberdeckungen für alle Platten angegeben sind. In den Bildern 37 bis 42 sind die derart ermittelten radialen Dehnungen auf den $x-, y$ - und $\xi$-Achsen dargestel1t. Die Bilder 43 bis 48 geben einen 
Ueberblick über die mittlere Dehnungsverteilung in den drei Achsrichtungen über die ganze Platte für ausgewählte Laststufen. Die Werte in n-Richtung lassen sich aus den symmetrisch liegenden Werten der $\xi-$ Richtung ablesen. Eine tabellarische Zusammenstellung aller gerechneten Dehnungen für alle Laststufen findet sich in Anhang in den Tabellen Al bis A6. Aus den Dehnungen der drei Seiten eines Dreiecks können die Hauptdehnungen und die Hauptdehnungsrichtungen ermittelt werden. Diese sind in den Bildern 49 bis 53 dargestellt.

Zur Bestimmung der mittleren Dehnung in der Biegebewehrung ist zu beachten, dass Bewehrungsebene und Messebene nicht zusammenfallen. Unter der Annahme des Ebenbleibens der Querschnitte reduziert sich die in der Messebene 'Oberseite' gemessene Dehnung um ca. 5\% für die y-Richtung und um ca. 15\% für die x-Richtung. Die Darstellung in den Bildern 37, bis 53 erfolgt ausschliesslich in den Messebenen.

Die Dehnungen, die in der Auswertung gemittelt wurden, wichen in den unteren Laststufen im Durchschnitt etwa um einen absoluten Betrag von $0.1 \%$ voneinander ab. Infolge der fortgeschrittenen Rissbildung traten bei höheren Lasten in einigen Fällen Unterschiede bis zu $2 \%$ auf.

\subsubsection{Betonstauchungen}

Die mittels Strain Gauges an der Plattenunterseite gemessenen Betonstauchungen sind in den Bildern 54 bis 59 laststufenweise in Funktion des Radius $r$ sowie in den Bildern 60 bis 65 messstellenweise in Funktion der Last $R$ aufgetragen. Die an der Stütze gemessenen Betonstauchungen sind in den Bildern 66 bis 69 in Abhängigkeit des polaren Winkels $\varphi$ enthalten.

\subsubsection{Stahldehnungen der Büge1}

Die in den Platten P 17 und P 22 an einigen Bügeln mit Strain Gauges gemessenen Stahldehnungen sind in den Bildern 70 und 71 dargestellt. Da pro Querschnitt nur ein einzelner Dehnmessstreifen aufgek lebt war, konnte der Einfluss einer möglichen Biegung im Bügel nicht ausgeschlossen werden.

\subsubsection{Zunahme der Plattendicke}

Die gemessene Zunahme der Plattendicke ist in den Bildern 72 bis 77 messstellenweise aufgezeichnet. Die Messung musste sehr sorgfältig durchgeführt werden, da sie sehr störungsanfällig war. In den höheren Laststufen sind einige Messungen ausgefallen, da die Lagerplättchen zum Anschlag des Messgerätes sich vom Beton lösten (Rissbildung).

Bei den Platten P 18, P 20 und P 22 wurde zusätzlich zur totalen Dicke über $180 \mathrm{~mm}$ auch noch von der Plattenoberseite bis in eine Tiefe von $50 \mathrm{~mm}$ gemessen, um eine Ablösung der oberen Bewehrung erkennen zu können. Diese Messung war nicht immer erfolgreich, weil es schwierig war, die Messbolzen anzubringen und das Messgerät anzusetzen. Im Bild 78 sind die beiden Messungen über 50 sowie über $180 \mathrm{~mm}$ für die Platte P 18 messstellenweise dargestellt.

\subsubsection{Mittlere Dehnungen in der Aussparung}

Die Messwerte der Deformetermessungen sind in den Bildern 79 bis 82 dargestellt. Auf die Darstellung der Messungen an der Stützseite direkt über der Stütze wurde verzichtet, da diese die Bruchfläche meist nicht tangierten. In den beiden unteren Ecken der Stützseite, in denen ein Bolzen aufgeklebt war, löste sich häufig ein Betonkeil, der weder mit dem Stützenteil noch mit dem Plattenteil direkt verbunden blieb. Die horizontale Dehnung am oberen Rand wurde nicht direkt gemessen, sondern aus den Vertikal- und Diagonaidehnungen und der unteren Horizontaidehnung berechnet und dann aufgezeichnet. Auf eine Mittelbildung wurde verzichtet, weil infolge unterschiedlicher Rissbildung die gemessenen Werte wesentlich voneinander abweichen konnten. Die Darstellung der Messwerte der Platte $\mathrm{P} 20$ wurde weggelassen, da in den Aussparungen nur unwesentliche Risse aufgetreten sind, und da der Durchstanzkegel nicht durch die Aussparungen verl ief. 


\subsubsection{Bewegungen des Stützenfusses}

Die vertikale Verschiebung und die Verdrehung des Stützenfusses wurden in den Versuchen $P 16$ bis $P 18$ und $P 22$ nur mit drei Messuhren gemessen. Diese Messungen erwiesen sich insbesondere zur Bestimmung der Rotation als unbefriedigend. Im Versuch $P 17$ fiel zudem eine Messuhr teilweise aus, so dass die Bewegung des Stützenfusses nicht mehr bestimmt werden konnte. Die Rotation um die y-Achse wurde in diesem Fall durch Integration der Krümmungen auf der $x$-Achse bestimmt, die Vertikalverschiebung durch Interpolation. Bei den Versuchen $P 19$ und P 20 ergaben sich gute Resultate, da drei überzählige Messungen vorhanden waren. Die relative Verdrehung der Stützenachse gegenüber der Plattenebene ist für die nur eine Symmetrieachse aufweisenden Platten P 16 bis $P 19$ im Bild 102 gegeben.

\subsubsection{Risse}

Die Risse wurden in bestimmten Abständen von der Stütze für jede volle Laststufe gemessen. Die Messstellen waren mit Bleistift markiert. Die Rissweiten in $0.01 \mathrm{~mm}$ wurden im allgemeinen senkrecht zur Rissrichtung angeschrieben (bei $P 16$ und $P 22$ noch nicht konsequent). Die Rissweiten sind in den Bildern 83 bis 88 angegeben und können mit einer Lupe gut gelesen werden.

\subsection{Allgemeines Tragverhalten}

Alle Platten wurden verformungsgesteuert bis zum Bruch belastet (Bild 21). Es wurden dabei folgende Phasen durchlaufen:

\subsubsection{Ungerissene Phase}

Die ungerissene Phase wird begrenzt von Belastungsbeginn und der Risslast. Diese Phase wurde nicht speziell untersucht.

\subsubsection{Risslast}

Die Risslast ist jene Last, bei der die ersten von Auge erkennbaren Risse auftreten. Die Bestimmung der Risslast gestaltete sich schwierig, weil die meisten Platten schon vor dem Belastungsbeginn unterschiedlich viele Schwindrisse aufwiesen. Diese gingen vor allem von den Aussparungsecken aus. Die beobachtete und auch im R-W-Diagramm deutlich als Knick erkennbare Risslast lag bei allen Platten - ausser bei der vorgespannten Platte $P 20$ - in der Grössenordnung von $100 \mathrm{kN}$. Bei der Platte $P 20$ traten die ersten Risse erst bei einer Last von $400 \mathrm{kN}$ auf. Auf dem Rissniveau wurde in allen Versuchen eine automatische, in $P 16, P 18$ und $P 22$ auch eine Deformetermessung durchgefürt. Die beobachteten Risslasten der Versuche $P 16$ bis $P 19$ entsprachen etwa jenen, die in früheren Versuchen [6] festgeste11t wurden. Die Streuung der Durchbiegungen beim Rissbeginn erklärt sich durch die unzulängliche Messung der Lagereinsenkung (siehe Abschnitt 4.1.8).

\subsubsection{Gerissene Phase}

Die gerissene Phase reicht von der Risslast bis zum Erreichen der Traglast. Diese Phase wurde gründlich beobachtet und ist in den folgenden Abschnitten eingehend beschrieben.

\subsubsection{Durchstanzen}

Bei allen Platten mit Ausnahme von $P 17$ trat der Bruch schlagartig ein. Er kündigte sich nur bei den $P l a t t e n ~ P 17$ und $P 22$ durch eine starke Zunahme der Durchbiegungen an. Im Versuch $P 17$ konnte vor dem eigentlichen Versagen eine starke Zunahme der Stützenneigung gegenüber der Plattenebene beobachtet werden (Bild 102). In allen Platten mit Ausnahme von P 17 trat ein Trennbruch in der Form eines Durchstanzkegels auf. Die Platte P 17 versagte durch Drehen der Stütze in Richtung der Aussparung (Bi1d 101). 


\subsubsection{Verhalten nach dem Durchstanzen}

Die Platten wurden nach dem Durchstanzen nochmals belastet. Dabei stellte sich ein neues Tragsystem ein, bestehend aus einer Kombination von Hängewerk und Dübelwirkung. Ausser bei der Platte $P$ 17, bei der ein Fliessplateau erreicht wurde, und bei der vorgespannten Platte $P 20$ war es in keinem Fall möglich, nochmals mehr als die Hälfte der Bruchlast aufzubringen. Meist ergaben sich in dieser Phase Probleme, da sich die Stütze aus der Vertikalen herausdrehte, so dass der Versuch abgebrochen werden musste. Die Durchstanzkegel wurden aus der Platte herausgetrennt und vermessen (Bilder 95 bis 100 ).

\subsection{Generelle Beobachtungen}

\subsubsection{Durchbiegungen}

Die in den Bildern 31 bis 36 dargestellten Profile weisen mit Ausnahme der Platte $P 17$ alle dieselbe Charakteristik auf: Die Krümung in radialer Richtung ist über dem Stützenrand konzentriert, und die Biegelinien verlaufen bis zum Rand praktisch gerade. In der Platte P 17 kann für höhere Laststufen eine negative Krümmung neben der Stuitze festgestellt werden. Die negative Durchbiegung auf der negativen $x$-Achse der Platten $P 17$ und $P 18$ resultiert aus der Verdrehung der Platte gegeniber der Stuitze. In den Bildern ist die Stuitzenachse immer vertikal eingezeichnet.

\subsubsection{Stahldehnungen der Biegebewehrung}

Die Fliessdehnung der Biegebewehrung wurde nur bei den Platten mit Schubbewehrung erreicht. Die Grenze des plastifizierten Bereichs lag in y-Richtung etwa bei 250 bis $500 \mathrm{~mm}$. In x-Richtung lagen die maximalen Dehnungen in den Platten $P 16$ bis $P 19$ im Bereich neben der Aussparung.

Die Stahldehnungen der Biegebewehrung lassen sich aus den mittleren Dehnungen der Messebene 'Oberseite' ermitteln ( $B i l d e r 37$ bis 48 ), wobei die unterschiedlichen Lagen von Messebene und Bewehrungsebene $z u$ berücksichtigen sind ( $B$ ild 20 ).

\subsubsection{Betonstauchungen}

Bei allen Platten wurde an der Plattenunterseite direkt neben der Stütze in den höheren Laststufen eine Abnahme der radialen Betonstauchungen beobachtet, während die tangentialen Stauchungen stark zunahmen. Die maximalen Stauchungen in radialer Richtung traten in den Platten mit Schubbewehrung und mit Vorspannung auf und betrugen in radialer Richtung $\mathrm{ca}$. $3 \% \mathrm{o}$, in tangentialer Richtung über $4 \%$ (Bilder 54 bis 65 ).

\subsubsection{Risse}

Die Platten wiesen häufig schon vor dem Versuchsbeginn Schwindrisse auf. Diese gingen vor allem von den Ecken der Aussparungen aus und folgten den obersten Bewehrungsstäben. Unter Last bildeten sich zuerst radiale, später tangentiale Risse. Vor allem die Platten mit Schubbewehrung waren in den höchsten Laststufen über der Stuitze jeweils sehr stark zerrissen. Die grössten Risse gingen immer von den Aussparungen aus. Diese Risse gingen in Tangentialrisse über, wobei die Rissbreite rasch abnahm und nach einem Abstand, der etwa der Plattendicke entsprach, noch etwa ein Viertel der ursprünglichen Breite betrug. Die grösste Rissweite ausserhalb des Eckbereichs betrug bei den nicht mit Schubbïgeln bewehrten Platten etwa $0.4 \mathrm{~mm}$, bei den schubbewehrten Platten wesentlich mehr als $1 \mathrm{~mm}$, mit Ausnahme der Platte $P$ 19, und bei der vorgespannten Platte etwa $0.6 \mathrm{~mm}$ (Bilder 83 bis 88 ).

\subsubsection{Hauptdehnungen}

Die Darstellung der Hauptdehnungen in den Bildern 49 bis 53 zeigt, dass die Rotationssymmetrie in dem Teil der Platte, der nicht von einer Aussparung gestört war, gut erhalten blieb. Im Stützenbereich der Platte P 22 kann ein orthotropes Verhalten festgestellt werden, das auf die unterschiedliche statische Höhe der beiden Bewehrungslagen zurückzuführen ist. 


\subsubsection{Stauchungen der Stütze}

Die auf der Stütze in z-Richtung gemessenen Betonstauchungen variierten über den Umfang zum Teil beträchtlich (Bilder 66 bis 69). Die grösste Stauchung wurde im Bereich der y-Achse gemessen, die kleinsten Werte auf der positiven und der negativen $x$-Achse. Die Stauchung im Bereich $x<0, y=0$ war bei den . Platten mit Schubbewehrung bei gleicher Last erheblich grösser als bei den Platten ohne Schubbewehrung. In der Platte $P 18$ war die Stuitze bezüglich der $y$-Achse annähernd symetrisch beansprucht. Interessant ist auch die in mehreren Platten beobachtete sprunghafte Zunahme der Betonstauchungen auf der Stütze im Bereich der $y$-Achse bei höheren Laststufen. Diese Umlagerungen traten zuweilen auch bei konstant gehaltener Last auf und gingen mit Sprüngen in der radialen (Abnahme) und tangentialen (Zunahme) Betonstauchung auf der Plattenunterseite einher.

\subsubsection{Zunahme der Plattendicke}

Eine Zunahme der Dicke um $0.03 \mathrm{~mm}$ entspricht einer Dehnung senkrecht zur Plattenebene von etwa $0.2 \%$. Falls diese Dehnung überschritten wird, dürfte die Platte mit Sicherheit diagonale Risse aufweisen. In den Platten $P 16$ bis $P 19$ konnte vor allem auf der y-Achse eine bedeutende Zunahme der. Plattendicke verzeichnet werden, die neben der Stütze im Maximum etwa $1 \mathrm{~mm}$ erreichte (Bilder 72 bis 78).

\subsubsection{Bruchfiguren}

Die Durchstanzkegel wurden aus den Platten herausgetrennt, um den genauen Verlauf der Bruchfläche erkennen zu können. Die Bewehrung wurde daher in der Bruchzone freigelegt und mit Hilfe eines Schneidbrenners durchgetrennt. Die Profile der Bruchflächen wurden in vier Schnitten am Durchstanzkegel sowie an der Platte ausgemessen und sind in den Bildern 95 bis 100 dargestellt. Die beiden an Platte und Kegel aufgenommenen Profile fallen nicht überall zusamen, da der Beton in der Bruchzone zum Teil in faust- bis tellergrossen Brocken (Bilder 89b, 91b und 93d), zum Teil bis zu Korngrösse zerbrochen war.

Die Platten $P 16$ und $P 22$ wurden entlang der $y$-Achse (P 16) und entlang der $x$-Achse (P 22) geschnitten, um über die Verteilung der Risse im Platteninneren Aufschluss zu erhalten. Es zeigte sich, dass die unverbügel te Platte $P 16$ ausserhalb des Durchstanzkegels völlig ungerissen war, während die mit Bügeln versehene Platte P 22 in diesem Bereich Risse aufwies (Bilder 89c, 94a und 94b).

\subsection{Spezielle Beobachtungen}

\subsubsection{Platte P 16}

Der Belastungsablauf ist aus Bild 23 ersichtlich. Die ersten Risse öffneten sich ungefähr bei $R=90 \mathrm{kN}$, ausgehend von den Ecken der Aussparung. In der Folge bildeten sich radiale Risse mit einem gegenseitigen Abstand von etwa $100 \mathrm{~mm}$ - was etwa dem Stababstand entspricht -, die sich rasch verzweigten und bis zum Rand ausdehnten (Bild 83). In der Aussparung verliefen die Risse unter einer Neigung von ca. $40^{\circ}$ schräg von den Seiten $\pm y$ in die Stützseite hinein. Ab Laststufe 6 (LS 6), d.h. bei $50 \%$ der Bruchlast, entstanden Tangentialrisse in einem Abstand von 200 bis $300 \mathrm{~mm}$ vom Stuitzenzentrum. Ab LS 10 zeigten die Dickenmessungen im Bereich der $y$-Achse wie auch die vertikalen Deformetermessungen in der Aussparung eine wesentliche Zunahme der Dehnung, was auf ein verstärktes Oeffnen der diagonalen, in der Platte verlaufenden Risse schliessen lässt, was in der Aussparung auch beobachtet werden konnte (Bilder 72 und 79). Ab LS 11 nahmen die radialen Betonstauchungen an der Unterseite ab, die tangentialen Stauchungen hingegen stärker zu (Bild 60). Die diagonalen Risse in der Aussparung erreichten zu diesem Zeitpunkt den unteren Rand, und die Platte wurde auch etwas weicher, was aus dem R-W-Diagram ersichtlich ist. Die auf der Stuitze gemessene Betonstauchung (Bild 66) nahm für $\varphi=90^{\circ}$ sprunghaft $z u$, dies gleichzeitig mit einer sprunghaften Abnahme der radialen und einer Zunahme der tangentialen Betonstauchungen auf der Plattenunterseite. In der letzten Laststufe erreichte der Maximalriss in der Ecke eine Oeffnung von ca. $1.5 \mathrm{~mm}$. Es konnte eine Schiebung im Riss in vertikaler Richtung festgestellt werden. In der Biegebewehrung wurde keine die Fliessdehnung überschreitende Dehnung gemessen. Die Risse in der Aussparung öffneten sich im Maximum ca. 0.35 mm. 
die automatischen Messungen ausgelöst werden konnten. Es löste sich ein Bruchkegel, der im Bereich der $y$-Achse eine Neigung von etwa $45^{\circ}$ aufwies, ausserhalb dieser Zone jedoch wesentlich flacher verlief (Bilder 89 und 95). In der Aussparung verlief die Bruchfläche auf der Seite $-y$ ausserhalb der beiden Bügel der Randverbügelung, auf der Seite ty zwischen den Bügeln hindurch. Die Platte wurde nach dem Bruch nochmals belastet. Die Randdurchbiegungen nahmen um $50 \mathrm{~m} \mathrm{zu}$, während der Widerstand noch $240 \mathrm{kN}$ erreichte.

Nachdem der Belastungsversuch abgeschlossen war, wurde die Platte bei $x=-110 \mathrm{~mm}$ entlang der $y$-Achse geschnitten. In der Schnittfläche konnten ausserhalb des Durchstanzkegels keine Risse in tangentialer Richtung festgestellt werden (Bild $89 \mathrm{C}$ ).

\subsubsection{Platte P 17}

Die Belastungsgeschichte dieser mit Bügeln bewehrten Platte ist im Bild 24 gegeben. Die ersten Risse gingen wiederum von den Ecken der Aussparung aus und begannen sich ca. ab $R=95 \mathrm{kN} z u$ öffnen. In der Folge entwickelten sich radiale Risse wie im Versuch $P 16$, die etwa bei $50 \%$ der Traglast im wesentlichen ausgebildet waren. Erste Tangentialrisse konnten schon in der LS $5(R=266 \mathrm{kN})$ festgestellt werden, und zwar in einem radialen Abstand von $200 \mathrm{~mm}$ von der Stütze. Weitere Tangentialrisse entstanden bei $300 \mathrm{~mm}$, dann bei $400 \mathrm{~mm}$ (Bild 84). Auch in der Aussparung bildeten sich ähnliche Risse wie in der Platte P 16. Allerdings waren diese eher stärker geneigt. Bei Laststufe $10(R=538 \mathrm{kN})$ betrug die Zunahme der Plattendicke bei der Stütze $0.35 \mathrm{~mm}$ bei einer gemessenen Bügeldehnung von ca. 1\%o (Bilder 73 und 70 ). Ab Laststufe 11 $(R=611 \mathrm{kN})$ konnte eine deutliche vertikale Verschiebung der Rissufer, besonders im Aussparungsbereich, beobachtet werden. Die radialen Stauchungen an der Plattenunterseite nahmen nun ab, die tangentialen Stauchungen wuchsen stärker (Bild 61). Die Durchbiegungen nahmen fortan stärker zu bei geringerer Lastzunahme. Die Stütze verdrehte sich stark gegenüber der Plattenebene, indem der Stützenfuss sich gegen die Aussparung hin bewegte ( $B$ ild 102). In der Laststufe 12 wurde die Fliessdehnung in der Bewehrung über der Stütze erreicht (Bild 38). Die Rissweite des grössten Risses in der Aussparung betrug nun ca. $0.5 \mathrm{~mm}$, und die Plattendicke hatte auf der $y$-Achse nahe der Stuitze etwa um denselben Betrag zugenomen (Bild 73). In der Laststufe 17 wurde in nächster Nähe der Stütze eine totale Zunahme der Plattendicke auf ca. 1 mm gemessen. Die Dehnung in den Bügein nahm bis zu diesem Niveau ungefähr linear mit der Last zu und wuchs nun plötzlich ausserordentlich stark (Bild 70). Nach der Zwischenlaststufe 18 konnte die Last nicht mehr weiter gesteigert werden. Es bildeten sich nun Risse auf der Unterseite der Platte etwa bei $x=-200$ bis $-400 \mathrm{~mm}$. Oben schlossen sich in derselben Region die Tangentiairisse wieder. Auf der Unterseite begann der Beton abzubröckeln, und die Ankerplättchen der Bügel kamen zum Vorschein. In der Laststufe 19 wurde sofort die Deformation konstant gehalten (anstatt zuerst zwei Minuten lang Last konstant) und sogleich eine volle Messung durchgeführt. Die Zunahme der Plattendicke betrug nun wesentlich mehr als $1 \mathrm{~mm}$ auf der $y$-Achse. An den entsprechenden Bügeln konnten über $2 \%$ o Dehnung gemessen werden. Die stützennahen Bügel auf der x-Achse wiesen maximale Dehnungen von nur $0.8 \%$ auf. Bei der nun folgenden Steigerung der Durchbiegung neigte sich die Stütze noch stärker gegenüber der Platte. Es bildete sich ein Mechanismus mit Schiebungen zwischen den Büge In bei $x>0$ und Rotationen bei $x<0$ (Bilder 90, 101 und 102). Die Durchbiegung nahm bei nur leicht abfallender Last auf ca. $35 \mathrm{~mm}$ zu. Die Deformation konnte nicht mehr weiter gesteigert werden, da die Lagerung instabil wurde. Daher wurde der Versuch abgebrochen. Der Durchstanzkegel konnte im Bereich $x<0$ herausgebrochen werden, indem die Rotation der Stütze verhindert wurde. Es waren keine Messungen mehr möglich. Die Bruchfläche verlief sehr steil und wurde senkrecht zur Plattenebene nur von drei bis vier Bügeln geschnitten (Bilder 90 und 96 ).

\subsubsection{Platte P 18}

Bild 25 gibt die Belastungsgeschichte der mit einer grossen Aussparung versehenen Platte P 18. Die Rissentwicklung nahm auch hier ihren Anfang in den Ecken der Aussparung. Zudem entstanden Biegerisse über der Stuitze senkrecht zum Rand. Bis zur Laststufe 7 bildeten sich strahlenförmig Radialrisse aus, ähnlich denjenigen der vorangehenden Platten. In der Aussparung bildeten sich früh je zwei parallele Risse, die an der Stützseite diagonal gegen die Stütze hin nach unten verliefen. Die Neigung betrug ca. $35^{\circ}$ (Bild $85 e$ ). Nach LS $4(R=190 \mathrm{kN})$ war das Rissbild fast vollständig ausgebildet. Die Plattendicke nahm nun in der $y$-Achse ganz wesentlich zu, ebenso die Relativverdrehung Platte - Stütze (Bilder 74 und 102). In der Laststufe 7 $R=258 \mathrm{kN}$ ) erreichten die radialen Betonstauchungen auf der Plattenunterseite wie auch die radialen mittleren Dehnungen auf der negativen $x$-Achse einen Maximalwert (Bild 39 und Tabelle A3). Die Inkremente 
änderten nun ihr Vorzeichen. Die gemessenen mittleren Dehnungen in horizontaler Richtung überschritten bis zum Bruch nirgends 2\%o. Die in der Aussparung nahe der Ecke gemessene mittlere Vertikaldehnung lag etwa bei $10 \%$ (Bild 81 ), was bei einer Messbasis von $141 \mathrm{~mm}$ einem Riss von ca. $1.4 \mathrm{~mm}$ entspricht.

Nach der Laststufe 9 wurde die Platte weiterbelastet. Bei einer Randdurchbiegung von $8.9 \mathrm{~mm}$ und einer Last von $370 \mathrm{kN}$ trat ein schlagartiger Bruch ein. Es löste sich ein Durchstanzkegel mit einer Neigung von ca. $45^{\circ}$ auf der $y$-Achse (Bilder 91 und 97). Nach dem Bruch wurde die Randdurchbiegung nochmals um ca. $50 \mathrm{~mm}$ gesteigert und damit noch ein Widerstand von $R=160 \mathrm{kN}$ erreicht.

\subsubsection{P1atte P 19}

Bild 26 zeigt den Belastungsablauf der mit Bügeln bewehrten Platte $P$ 19. Die Platte wies vor dem Belastungsbeginn schon erhebliche Schwindrisse auf. Es wurde ungefähr auf das Rissniveau der Platte P 17 gefahren und dort eine Messung vorgenomen. Dabei entstanden kaum neue Risse. In der Aussparung waren die Risse auf das oberste Drittel der Plattendicke beschränkt. Das sich in der Folge einstellende Rissbild entsprach ungefähr demjenigen von $P$ latte $P$ 17, die Risse waren jedoch eher besser verteilt (Bild 86 ). Ab Laststufe $9(R=450 \mathrm{kN})$ war die radiale Betonstauchung in Stützennähe an der Unterseite rückläufig (Bild 63). Gleichzeitig konnte in den direkt neben der Stitze liegenden Messstel len eine deutliche Zunahme der Plattendicke beobachtet werden (Bild 75). Die Stauchung in der Stütze im Bereich $\varphi=90^{\circ}$ nahm gleichzeitig sprunghaft zu (Bild 69). Wie aus Bild 26 zu erkennen ist, wurde die Platte ab LS 11 ( $R=630 \mathrm{kN}$ ) wesentlich weicher. Die Biegebewehrung in $y$-Richtung wurde ab LS 11 , diejenige in $x$-Richtung ab LS 11 plastifiziert (Bild 40). In der Aussparung traten zwar auch Risse auf, diese waren aber relativ gut verteilt, und es öffnete sich kein Riss mehr als etwa $0.4 \mathrm{~mm}$. Die relative Verdrehung Platte - Stütze nahm linear mit der Last $z u$, und zwar neigte sich der Stützenfuss gegen die Aussparung (Bild 102).

In der 13. Laststufe wurde eine Last $R$ von $800 \mathrm{kN}$ erreicht. Der Bruch trat nach weniger als zwei Minuten während dem Konstanthalten der Last schlagartig ein. Der Bruch ging vom unverbügelten Bereich $x<0$ aus und breitete sich von dort rund um Stütze und Aussparung aus. Daher änderte sich auch die Stuitzenneigung, und der Stützenfuss drehte sich nun von der Aussparung weg (Bild 102). Nach dem Eintreten des Bruchs konnte bei einer maximalen Durchbiegung von $25 \mathrm{~mm}$ noch ein Widerstand von $R=390 \mathrm{kN}$ aktiviert werden. Die Bruchfläche umschloss sowohl die Aussparung als auch den gesamten verbügelten Bereich. Auf der von der Aussparung abgewandten Seite verlief sie ähnlich wie in P 16. Die Neigung der Bruchfläche betrug ca. $30^{\circ}$ bis $35^{\circ}$ (Bilder 92 und 98 ).

\subsubsection{Platte P 20}

Die vorgespannte Platte $P 20$ wurde wie folgt belastet: Zuerst wurde an allen 12 Kabeln die volle Vorspannkraft aufgebracht. Dann wurde allein mit den vier zusätzlichen Kolben auf $R=392 \mathrm{kN}$ gefahren, um eine direkte Belastung an den Ankerstellen der Vorspannkabel anzubringen. Anschliessend wurde mit den anderen acht Kolben weiterbelastet (Bild 27). Die Platte verhielt sich während dieses ganzen Vorgangs ungerissen. Auf der Unterseite konnten nach dem Spannen der Kabel (Randdurchbiegung $w=-3.3 \mathrm{~mm}$ ) keine Risse entdeckt werden. Die ersten Risse wurden erst bei LS $5(R=394 \mathrm{kN})$ deutlich sichtbar, worauf die Platte ein wesentlich weicheres Verhalten aufwies (Bild 87). Es entstanden zuerst radiale Risse in der ganzen Platte und ab LS 7 auch tangentiale Risse im inneren Bereich bis zu den Aussparungen. In der Laststufe 11 war das Rissbild vollständig ausgebildet. Die Plattendicke nahm ab Laststufe $9(R=560 \mathrm{kN}$ ) stark zu, und zwar bis zu einem radialen Abstand von der Stütze von $300 \mathrm{~mm}$. Ausserhalb dieses Bereichs konnte bis zum Bruch keine nennenswerte Zunahme der Plattendicke beobachtet werden (Bild 76). In x-Richtung wurde in der Laststufe 14, in $y$-Richtung in der Laststufe 20 erstmals eine die Fliessdehnung überschreitende Dehnung gemessen (Bild 41). Ausserhalb eines Radius von $500 \mathrm{~mm}$ in $x$-Richtung und von $700 \mathrm{~mm}$ in $y$-Richtung war die Platte auch auf der Oberseite immer gedriickt. Die Betonstauchungen nahmen bis zur zweitletzten Laststufe vor dem Bruch kontinuierlich mit der Last zu, mit einem deutlichen Knick beim Ueberschreiten der Risslast (Bild 64). In der letzten gemessenen Laststufe war in praktisch allen Strain Gauges eine deutliche Abnahme der Stauchungen $z u$ verzeichnen. Auf der $\xi$-Achse wurden in tangentialer Richtung maximale Stauchungen von 4\%o gemessen. Die mittleren Kabelkräfte nahmen bis zum Bruch mit der Last zu und erreichten maximal $P=200 \mathrm{kN}$, was einer Zunahme um 13\% entspricht ( $B i l d 30$ ). In den Aussparungen entstanden vor allem vertikale, einige diagonale und später auch noch horizontale Risse auf der Höhe der Spannkabel. Alle Risse waren 
von geringer Breite und waren auf die oberen 5/6 der Plattendicke beschränkt (Bild 93C).

Der Bruch trat schlagartig während des Konstanthaltens der Last nach LS 27 ein, noch bevor die automatische Messung ausgelöst worden war. Die dabei kurzfristig erreichte Last R betrug $920 \mathrm{kN}$. Nach dem Bruch wurde die Platte nochmals belastet. Es bildete sich ein Hängewerk aus, wobei bei einer maximalen Randdurchbiegung von $45 \mathrm{~mm}$ noch eine Last von $600 \mathrm{kN}$ aufgenommen werden konnte (Bild 29). Der Kabelkraftzuwachs betrug dabei 17\% (Bild 30). Beim Heraustrennen des Durchstanzkegels zeigte sich, dass zwischen P1atte und Stuitze zwei Bruchflächen vorhanden waren, eine innere steile und eine äussere flache (Bilder 93 und 99). Der zwischen den beiden festen Körpern liegende Beton war in scheibenförmige, ca. 200 bis $300 \mathrm{~mm}$ grosse Betonbrocken zerbrochen, die sich leicht von Hand entfernen liessen (Bild 93d).

\subsubsection{Platte $P 22$}

Die Platte P 22, mit Bügelbewehrung und ohne Aussparung, wurde als Demonstrationsobjekt anlässlich eines Fortbildungskurses bis zum Bruch geprüft. Aus diesem Grund wurde sie in zwei Etappen belastet (Bild 28). Die ersten Risse traten etwa bei $R=110 \mathrm{kN}$ auf und verliefen tangential und radial etwas ausserhalb des Stiutzenrandes. Es bildeten sich rasch weitere Radialrisse und Rissverzweigungen. Etwa ab LS 9 waren die radialen Risse vollständig ausgebildet (Bild 88 ). In der Laststufe 7 ( $R=365 \mathrm{kN}$ ) nahm die Plattendicke in den beiden am nächsten bei der Stütze liegenden Messstellen um je ca. $0.1 \mathrm{~mm}$ und fortan regelmässig weiter zu (Bild 77). In $x$-Richtung wurde in der Laststufe 8 und in y-Richtung in der Laststufe 12 erstmals eine die Fliessdehnung überschreitende Dehnung in der Biegebewehrung gemessen (Bild 42). Gleichzeitig nahmen auch die Randdurchbiegungen stärker $z u$. Ab Laststufe 14 ( $R=785 \mathrm{kN}$ ) nahmen die radialen Betonstauchungen auf der Unterseite ab (Bild 65). Es bildeten sich nun noch weitere tangentiale Risse bis zu einem Radius von etwa $500 \mathrm{~mm}$. Nach der Laststufe 14 wurde entlastet, die vier zusätzlichen Kolben wurden eingebaut und die Randdurchbiegungen wieder auf den fruheren Wert gefahren. Rissbild, Last und Verformung blieben gegenüber dem Lastsystem mit acht Kolben praktisch unverändert.

Nach der Laststufe 18 wurde der Versuch unterbrochen, um den Bruch während einer Demonstration vor Fachleuten herbeizuführen. Dabei konnten nur noch einige ausgewähl te Messungen durchgeführt werden. Die gemessenen Stahldehnungen in den Bügeln nahmen etwa linear mit der Last zu und erreichten im Maximum bei $y=160 \mathrm{~mm}$ 2.5\% (zugehörige Zunahme der Plattendicke: $0.8 \mathrm{~mm}$, Bilder 71 und 77). Mit zunehmendem Abstand von der Stütze wurde eine abnehmende Dehnung in den Bügeln gemessen. Bis zur Laststufe 23 wurde eine Zunahme der Dicke in $x=-160 \mathrm{~mm}$ um ca. $2 \mathrm{~mm}$ beobachtet. Die Höchstlast wurde in der Laststufe $25 \mathrm{mit} 1044 \mathrm{kN}$ erreicht. In der nächsten Laststufe konnte nur noch die Durchbiegung gesteigert werden, die Last hingegen ging leicht zurück.

Der Bruch trat während der weiteren Steigerung der Durchbiegung bei einem Gesamtweg von ca. $38 \mathrm{~mm}$ ein. Dabei löste sich ein sehr steiler Durchstanzkegel aus der Platte. Die Bruchfläche war ca. $75^{\circ}$ bis $80^{\circ}$ geneigt und verlief innerhalb des nicht mehr verbügelten Bereichs um die Stütze. Die Bügel, von denen keiner über die Stütze führte, blieben alle mit der Platte verbunden (Bilder 100 und 94). Die Wiederbelastung nach dem Bruch ergab eine Resttraglast $R$ von ca. $500 \mathrm{kN}$. Im Schnitt entlang der $x$-Achse zeigte sich ein fächerförmiges Rissbild, ausgehend von der Stütze und den der Stütze am nächsten liegenden Ankerplättchen. Ausserhalb von $400 \mathrm{~mm}$ vom Stützenzentrum konnten keine Risse mehr entdeckt werden (Bilder 94a und 94b). 


\section{Zusammenfassung}

In Flachdecken sind oft direkt neben den Stützen Aussparungen erforderlich, da Installationen meistens in vertikaler Richtung den Stiutzen entlang geführt werden. Dadurch wird der Schubwiderstand der Verbindung Stuitze - Platte geschwächt. Zur Verstärkung dieses Bereichs kann nebst anderen Massnahmen, wie z.B. Verwendung von Stahlelementen, auch eine vertikale Schubbewehrung in Form von Bügeln angeordnet werden.

Zum besseren Verständnis des Schubtragverhaltens von Flachdecken mit Aussparungen wurden am Institut für Baustatik und Konstruktion der ETH Zürich sechs Versuche durchgeführt. Die Versuchsparameter waren:

- Grösse der Aussparung

- Schubbewehrung (Menge und Anordnung)

- Längsbewehrung (schlaff und vorgespannt ohne Verbund)

Ein direkter Vergleich mit früheren Durchstanzversuchen an Flachdecken ohne Aussparungen [6] ist möglich.

Die Platten wurden verformungsgesteuert bis zum vollständigen Versagen des Stiutzenbereichs belastet. Dabei murden die Belastung, die Durchbiegungen, mittlere Dehnungen, lokale Betonstauchungen, Zunahmen der Plattendicke, Stahldehnungen in den Bügeln, Kabelkräfte und Rissweiten gemessen.

In funf Platten trat der Bruch schlagartig ein. Es bildete sich ein sauberer Trennbruch zwischen dem Durchstanzkegel und der Platte. Bei einer mit Bügeln bewehrten Platte führte die Bruchfläche um die Aussparung herum. Die andere schubbewehrte Platte mit Aussparungen wies ein duktiles Versagen auf, indem die relative Verdrehung zwischen Stütze und Plattenebene bei ungefähr konstanter Last ständig zunahm. Eine Plastifizierung der oberen Bewehrung konnte nur bei den mit Bügeln bewehrten und bei der vorgespannten Platte festgeste1lt werden.

Der Einfluss der Parameter lässt sich wie folgt beschreiben:

Die Reduktion des Schubtragwiderstandes hängt in erster Linie von der Breite der Aussparung in tangentialer Richtung ab.

Mit einer Schubbewehrung kann die Durchstanzlast erheblich gesteigert werden. Es ist nicht notwendig, den ganzen Stützenbereich mit Bügeln zu versehen. Vielmehr können Bügel konzentriert an den Stellen mit besonders hoher Schubbeanspruchung angeordnet werden, wenn die Lasteinleitung mit einem geeigneten Modell untersucht wird. Das Rissbild in der Aussparung kann durch eine enge Verbügelung des Randes günstig beeinflusst werden.

In vorgespannten Decken wird die Durchstanzlast durch Aussparungen, die ausserhalb der Vorspannbahnen angeordnet werden, kaum beeinflusst. 
Résumé

Dans les dalles en béton armé ou précontraint il faut souvent prévoir des évidements à proximité des piliers pour faire place aux installations. Leur influence négative sur la résistance au poinçonnement doit souvent être compensée par des renforcements métalliques spéciaux ou par des armatures de poinçonnement.

Dans le but d'approfondir les connaissances,sur le comportement au poinçonnement des dalles avec évidements, six dalles ont été testěes à l'Institut de Statique et de Construction de l'Ecole Polytechnique Fédérale de Zurich. Les paramètres étudiés sont:

- la dimension de l'évidement

- l'armature de cisaillement (quantité et disposition)

- l'armature de flexion (normale et précontrainte sans injection)

Des essais semblables ont dẹjà êté effectués avec des dalles sans évidement [6], une comparaison est donc possible.

Les dalles ont êté menées à la charge de rupture en contrôlant les déformations. Les grandeurs suivants ont été mesurées: les charges, les déplacements, les déformations moyennes, les dēformations locales du béton, l'augmentation d'épaisseur de la dalle, les déformations des étriers, les forces dans les cábles de précontrainte et la largeur des fissures.

Dans cinq cas la rupture est intervenue soudainement par poinçonnement. La surface de séparation qui s'est formée entre le cône de poinçonnement et la dalle était, dans ces cas là, nette. Dans le cas d'une dalle armée avec des étriers, la surface de séparation passait en dehors de l'évidement. Dans le cas d'une autre dalle armée avec une armature de poinçonnement, il n'y a pas eu de séparation complète entre le cône de poinçonnement et la. dalle. Une fois la charge ultime atteinte, on a pu observer une rotation de la dalle par rapport au pilier avec un comportement assez ductile. Une plastification importante de l'armature de flexion a pu être observée seulement dans les dalles qui ont une armature de poinçonnement ou dans les dalles qui sont précontraintes.

L'influence des paramètres, qui ont étẻ variês, peut être décrite comme suit:

La réduction de la charge de poinçonnement dépend en premiēre ligne de la largeur de l'évidement dans la direction tangentielle.

La rêsistance au poinçonnement peut être augnentée considérablement grâce à des étriers. Leur disposition peut tenir compte des concentrations des efforts de cisaillement. Dans ce cas il faut analyser le système porteur avec un modèle idoine.

Une armature de cisaillement disposée sur les bords de l'évidement peut limiter la fissuration sur ses côtés.

La charge de poinçonnement de dalles précontraintes suivant deux directions n'est pratiquement pas réduite par les évidements situés en dehors des bandes de précontrainte. 
Nel caso di solai piani devono spesso essere lasciati dei risparmi in prossimità del pilastro per far posto alle istallationi previste. Per sopperire all'indebolimento del collegamento solaio - pilastro da essi provocato si devono talvolte prevedere speciali costruzioni metalliche o armature al taglio.

Per migliorare le conoscienze sul comportamento al taglio di solai piani con risparmi sono state effettuate sei esperienze nel laboratorio dell'Istituto di statica delle costruzioni al Politecnico Federale di Zurigo. Le variabili delle esperienze erano:

- dimensioni del risparmio

- armatura al taglio (quantità e disposizione)

- armatura alla flessione (normale o precompressa senza aderenza).

Analoghe esperienze con solette senza risparmi sono state effettuate precedentemente [6], un confronto è pertanto possibile.

Le prove sono state caricate fino alla rottura regolando la deformazione. Sono stati misurati $i$ carichi e le reazioni, gli spostamenti vertivali, le deformazioni medie, le deformazioni locali del calcestruzzo, l'aumento di spessore della piastra, le deformazioni delle staffe, le forze nei cavi di precompressione e l'apertura delle fessure.

In cinque casi la rottura si è verificata per punzonamento. In queste prove il cono sovrastante il pilastro si è separato improvvisamente dal resto della piastro lungo una superficie ben delimitata. Nel caso di una prova provvista di staffe tale superficie racchiudeva completamente il risparmio. Nel caso di un'altra prova con armatura al taglio non si è verificata una separazione completa fra cono e piastra. Raggiunto i) carico ultimo la piastra ha cominciato a ruotare attorno al pilastro dimostrando un comportamento relativamente duttile. Un importante snervamento dell'armatura superiore è stato osservato solo in prove provviste di armatura al taglio o di cavi di precompressione.

L'influsso delle variabili puõ essere cosł descritto:

La riduzione del carico di punzonamento dipende essenzialmente dalla dimensione del risparmio lungo il perimetro del pilastro.

La resistenza al punzonamento puõ essere notevolmente aumentata mediante staffe. Esse possono anche essere disposte solo nelle zone di maggior concentrazione degli sforzi. In tal caso il sistema portante deve essere analizzato mediante un modello idoneo. Un' armatura al taglio disposta sui bordi del risparmio contribuisce a limitare la fessurazione sulle superfici dello stesso.

Ne1 caso di solai precompressi in due direzioni il carico di punzonamento non viene praticamente influenzato da risparmi disposti al di fuori delle corsie precompresse. 
Openings through flat slabs are often required in the column area due to the utilities and wiring along the columns. Consequently, the punching shear strength of the slab - column connection is reduced. An increase in the shear strength is attainable by the use of steel elements or vertical stirrups.

In order to obtain further information about the structural behaviour of flat slabs with openings in the column area, a series of tests has been executed at the Institute of Structural Engineering. These were the main parameters:

- size of the opening

- shear reinforcement (quantity and layout)

- flexural reinforcement (ordinary steel and unbonded prestressing steel).

A direct reference to earlier punching tests on flat slabs without openings [6] is possible.

A11 slabs were loaded and observed until complete failure. In order to observe the structural behaviour, the loading, deflections, medium strains, local concrete strains, growth of slab thickness, strain of steel in stirrups, tendon forces and crack widths were measured.

Five slabs failed suddenly in a brittle way. There was a separation fracture between column and slab. One of these slabs with vertical stirrups in the column area failed beyond the opening in separation fracture. Another slab with stirrups failed ductily: the load remained almost constant while the relative angle between column axis and slab increased. Yielding of the reinforcement tendons were observed only in the slabs with shear reinforcement and the slab with prestressing.

The influence of the parameters can be described as follows:

The reduction of shear resistance by holes adjacent to the column is mainly influenced by the width of the opening in tangential direction.

A shear reinforcement raises the shear resistance considerably. Instead of supplying the whole column area with stirrups, it may be sufficient to lay out the stirrups at the positions submitted to the highest shear stress due to the opening. The load introduction has to be checked by means of an adapted model. A narrow space between the stirrups along the edges of the opening is favourable for the crack pattern.

If the openings are outside of the prestressing cable strips, the shear capacity of prestressed slabs is hardly reduced. 


\section{Verdankungen}

Der vorliegende Bericht wurde im Rahmen des Forschungsprojektes "Vorgespannte Platten" am Institut für Baustatik und Konstruktion der Eidgenössischen Technischen Hochschule Zürich ausgearbeitet. Für die grosszügige finanzielle Unterstützung dieses Projektes möchten die Verfasser folgenden Institutionen und Firmen aufrichtig danken:

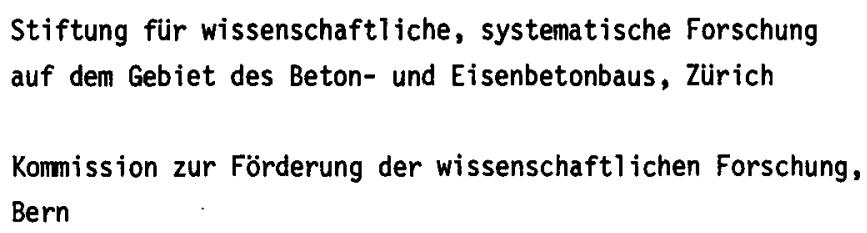

Das Forschungsprojekt wird von einer Beratenden Kommission begleitet, der die folgenden Herren angehören: Dr. M. Birkenmaier, Prof. R. Favre, Dr. M. Ladner, P. Matt, dipl. Ing., K. Messerli, dipl. Ing., Dr. P. Ritz, E. Setz, dipl. Ing., Dr. R. Wolfensberger.

Innen allen sei für ihr Mitwirken auch an dieser Stelle bestens gedankt.

Ein spezieller Dank gilt der

von Moos Stah1 AG, Luzern,

die sämtliche Stähle für die schlaffe Bewehrung der Versuchskörper fertig bearbeitet und kostenlos zur Verfugung gestellt hat.

Ein weiterer Dank gilt den Firmen

$$
\text { Losinger } A G, \text { Bern }
$$

für die Anfertigung der Bügel

Stahlton $A G$, Zürich

für die Lieferung der Spannkabel und die Ausführung der Spannarbeiten.

Bei der Versuchsdurchführung haben die Herren K. Bucher, C. Navarra, cand. Ing., und C. Vallecilla, dipl. Ing., mitgearbeitet. Herr M. Baumann, dipl. Ing., hat die messtechnischen Probleme bearbeitet. Für die Gestaltung des Berichtes und die Herstellung der Zeichnungen waren die Herren G. Göseli und L. Sieger besorgt, Frl. S. Burki schrieb die Druckbogen. Für ihre Mitarbeit sei den Genannten und auch anderen, die zu erfolgreichen Durchführung der Versuchsserie beigetragen haben, bestens gedankt.

Herrn R. Caflisch, dipl. Ing. und Adm. Institutsleiter, möchten wir speziell für seine Ratschläge und seine Unterstützung während der Versuchsvorbereitungen sowie für seine organisatorische und administrative Betreuung des Forschungsprojektes danken.

Schliesslich möchten wir auch noch den Herren Dr. J. Pralong und Dr. W. Brändli einen speziellen Dank aussprechen. Ihre Arbeiten und ihre in den vorangehenden Versuchsserien gemachten Erfahrungen haben zum Gelingen der Versuchsserie beigetragen. 


\section{Geometrische Grössen}

d

h

$\Delta \mathrm{h}$

$r$

W

x

y

$z$

$\xi$

$A_{\text {eff }}$

$\phi$

$\varphi$

$\rho$

\section{Kraftgrössen}

F

p

$P_{0}$

$\Delta \mathbf{P}$

R

$R_{u}$

Verformungen

$\begin{array}{ll}\delta_{g l} & \text { Gleichmassdehnung schlaffer Bewehrungsstahl } \\ \varepsilon & \text { Dehnung, allgemein } \\ \dot{\varepsilon} & \text { Dehnungsgeschwindigkeit } \\ \varepsilon_{c u} & \text { Betonstauchungen beim Bruch (Zylinderversuch) } \\ \varepsilon_{s} & \text { Dehnung der schlaffen Bewehrung } \\ \varepsilon_{s h} & \text { Dehnung der schlaffen Bewehrung bei Verfestigungsbeginn } \\ \varepsilon_{s y} & \text { Dehnung der schlaffen Bewehrung bei Fliessbeginn } \\ \varepsilon_{x} & \text { Dehnung in Richtung der x-Achse } \\ \varepsilon_{y} & \text { Dehnung in Richtung der } y \text {-Achse } \\ \varepsilon_{z} & \text { Dehnung in Richtung der } z \text {-Achse } \\ \varepsilon_{\xi} & \text { Dehnung in Richtung der } \xi \text {-Achse } \\ \varepsilon_{r} & \text { Dehnung in radialer Richtung } \\ \varepsilon_{\phi} & \text { Dehnung in tangentialer Richtung }\end{array}$

statische Höhe

Plattendicke

Zunahme der Plattendicke

Koordinatenachse

Verschiebung in Richtung der z-Achse, Durchbiegung

Koordinatenachse

Koordinatenachse

Koordinatenachse

Koordinatenachse

Koordinatenachse

effektive Stabquerschnittsfläche (Gewichtsmessung)

Durchmesser

polare Winkelkoordinate

geometrischer Bewehrungsgehalt

Kraft

Vorspannkraft

Vorspannkraft bei Randdurchbiegung $w=0$

Spannkraftzuwachs

Reaktion am Stützenfuss, Last

Bruchlast 


\section{Festigkeitswerte, Spannungen}

$E_{S} \quad$ Elastizitätsmodul des schlaffen Bewehrungsstahls

$\mathrm{E}_{\mathrm{p}} \quad$ Elastizitätsmodul des Spannstahls

$E_{c} 0.5$ Tangentenmodul des Betons für $\sigma_{c}=0.5 \mathrm{~N} / \mathrm{mm}^{2}$

$E_{c} 0.5 \div 10.0$ Sekantenmodul des Betons für $\sigma_{c}=0.5 \div 10.0 \mathrm{~N} / \mathrm{mm}^{2}$

$\sigma_{c} \quad$ Spannung im Beton

$\sigma_{p} \quad$ Spannung im Spannstahl

$\sigma_{s} \quad$ Spannung im schlaffen Bewehrungsstahl

$f_{c} \quad$ Zylinderdruckfestigkeit des Betons

$f_{\mathrm{CW}} \quad$ Würfeldruckfestigkeit des Betons

$f_{c t} \quad$ Betonzugfestigkeit

$f_{p y} \quad$ Streckgrenze des Spannstahis

$f_{\text {st }} \quad$ Zugfestigkeit des schlaffen Bewehrungsstahls (dynamisch)

$f_{\text {sy, stat Fliesspannung des schlaffen Bewehrungsstahis (statisch) }}$

$f_{\text {sy, dyn }} \quad$ Fliesspannung des schlaffen Bewehrungsstahls (dynamisch)

\section{Allgemeine Bezeichnungen}

LS

Laststufe

MS Messstelle

P Platte

n Anzahl Proben

" Zoll

$\% \quad 0.01=10^{-2}$

$\% \quad 0.001=10^{-3}$

Indizes

$\begin{array}{ll}\text { c } & \text { Beton } \\ \text { p } & \text { Vorspannung } \\ s & \text { schlaffe Bewehrung } \\ t & \text { Zug- } \\ y & \text { Fliess- } \\ r & \text { radial } \\ \varphi & \text { tangential } \\ u & \text { Bruch } \\ \text { stat } & \text { statisch } \\ \text { dyn } & \text { dynamisch } \\ \text { sup } & \text { oben } \\ \text { inf } & \text { unten }\end{array}$




\section{Literaturverzeichnis}

[1] Ritz P., Marti P., Thürl imann B.:

"Versuche über das Biegeverhalten von vorgespannten Platten ohne Verbund", Institut für Baustatik und Konstruktion, ETH Zürich, Versuchsbericht $\mathrm{Nr}$. 7305-1, Juni 1975, Birkhäuser Verlag Basel und Stuttgart.

[2] Marti P., Thürl imann B.:

"Fliessbedingung für Stahl beton mit Berücksichtigung der Betonzugfestigkeit", Institut für Baustatik und Konstruktion, ETH Zürich, Bericht Nr. 67, 1977, Birkhäuser Verlag Basel und Stuttgart.

[3] Marti P., Ritz P., Thürlimann B.:

"Prestressed Concrete Flat Slabs", Institut für Baustatik und Konstruktion, ETH Zürich, Bericht Nr. 68, 1977, Birkhäuser Verlag Basel und Stuttgart.

[4] Marti P., Pralong J., Thürlimann B.:

"Schubversuche an Stahlbetonplatten", Institut für Baustatik und Konstruktion, ETH Zürich, Versuchsbericht Nr. 7305-2, September 1977, Birkhäuser Verlag Basel und Stuttgart.

[5] Ritz P.:

"Biegeverhalten von Platten mit Vorspannung ohne Verbund", Institut für Baustatik und Konstruktion, ETH Zürich, Bericht Nr. 80, 1978, Birkhäuser Verlag Basel und Stuttgart.

[6] Pralong J., Brändli W., Thürl imann B.:

"Durchstanzversuche an Stahlbeton- und Spannbetonplatten", Institut für Baustatik und Konstruktion, ETH Zürich, Versuchsbericht Nr. 7305-3, 1979, Birkhäuser Verlag Basel und Stuttgart.

[7] Marti P.:

"Plastische Berechnung von Stahlbeton", Institut für Baustatik und Konstruktion, ETH Zürich, Bericht Nr. 104, 1980, Birkhäuser Verlag Basel und Stuttgart.

[8] Brändli W. , Muiller F.X., Thürlimann B.:

"Bruchversuche an Stahlbeton- und Spannbetonplatten bei Rand- und Eckstuitzen", Institut für Baustatik und Konstruktion, ETH Zürich, Versuchsbericht Nr. 7305-4, Birkhäuser Verlag Basel und Stuttgart.

[9] Praiong J.:

"Poinçonnement symétrique des planchers-dalles", Institut für Baustatik und Konstruktion, ETH Zürich, Bericht Nr. 131, 1982, Birkhäuser Verlag Basel und Stuttgart.

[10] Bränd]i W.:

"Durchstanzen von Flachdecken bei Rand- und Eckstützen", Institut für Baustatik und Konstruktion, ETH Zürich, in Bearbeitung.

[11] Joint ASCE-ACI Task Committee 426:

"The Shear Strength of Reinforced Concrete Members; Slabs", ASCE Journal of the Structurat Division No ST8, Vol. 100, August 1974, pp. 1559-1562.

[12] Elstner R.C., Hognestad E.:

"Shearing Strength of Reinforced Concrete Slabs", Journal of the American Concrete Institute (ACI), No. 1 , Vol. 53 , July 1956, pp. 29-58.

[13] Moe J.:

"Shearing Strength of Reinforced Concrete Slabs and Footings under Concentrated Loads",

Portland Cement Association, Development and Department Bulletin D 47, Skokie, III., USA, April 1961 .

[14] Mowrer R.D., Vanderbilt M.D.:

"Shear Strength of Leightweight Aggregate Reinforced Concrete Flat Plates", ACI Journal, Proceedings No. 11, Vol. 64, No. 11, November 1967, pp. 722-729.

[15] Zaidi S.T.H., Roll F.:

Shear Resistance of Perforated Reinforced Concrete Slabs", University of Pennsylvania, School of Mech. Engineering, Philadelphia, November 1968.

[16] Andrä H.P.:

"Zum Tragverhalten von Flachdecken mit Dübel leisten-Bewehrung im Auflagerbereich", Beton- und Stahlbetonbau, Vol. 76, Hefte 3 und 4, März und April 1981, Verlag Wilhelm Ernst + Sohn, Berlin, S. 53-57 und 100-107. 


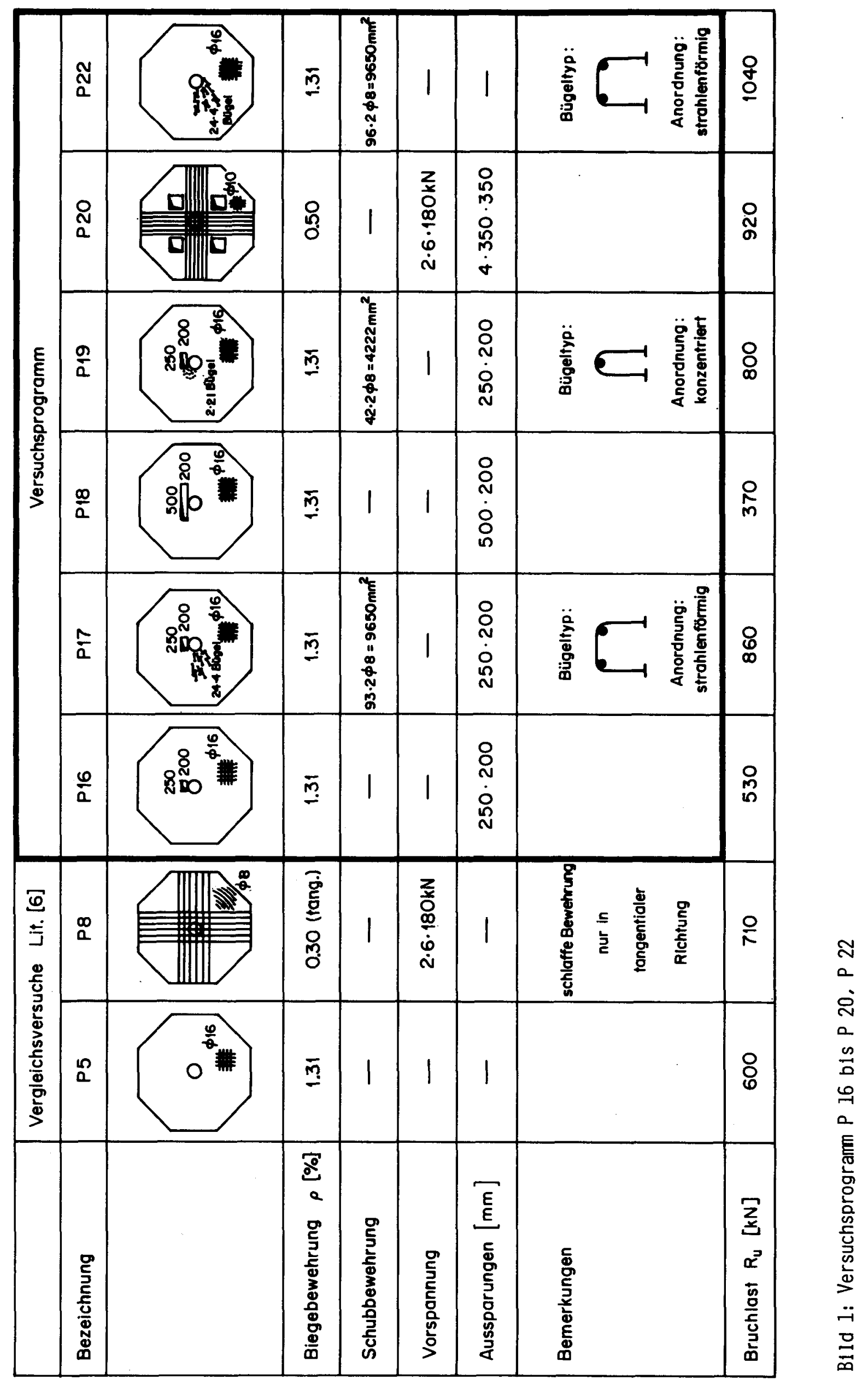



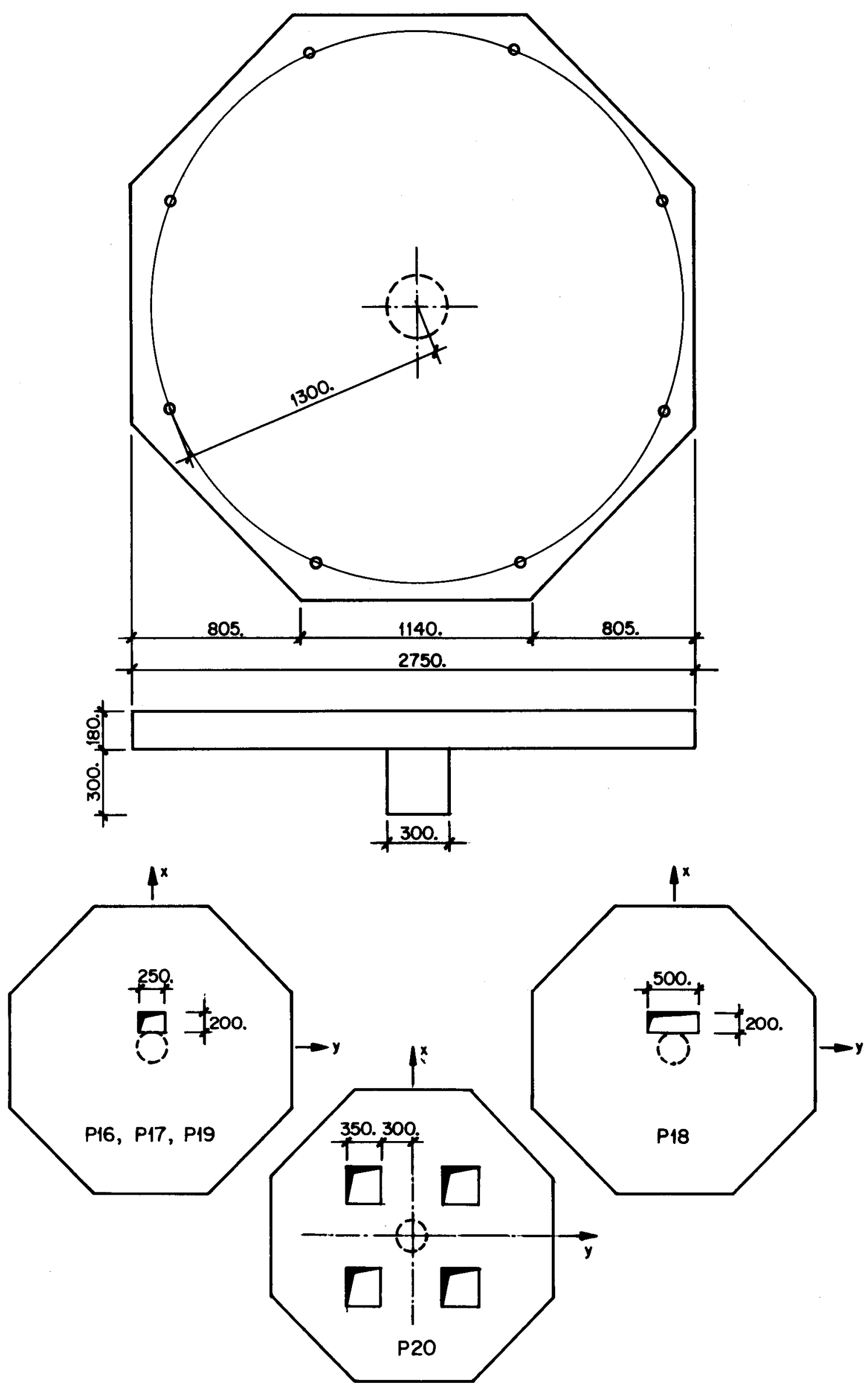


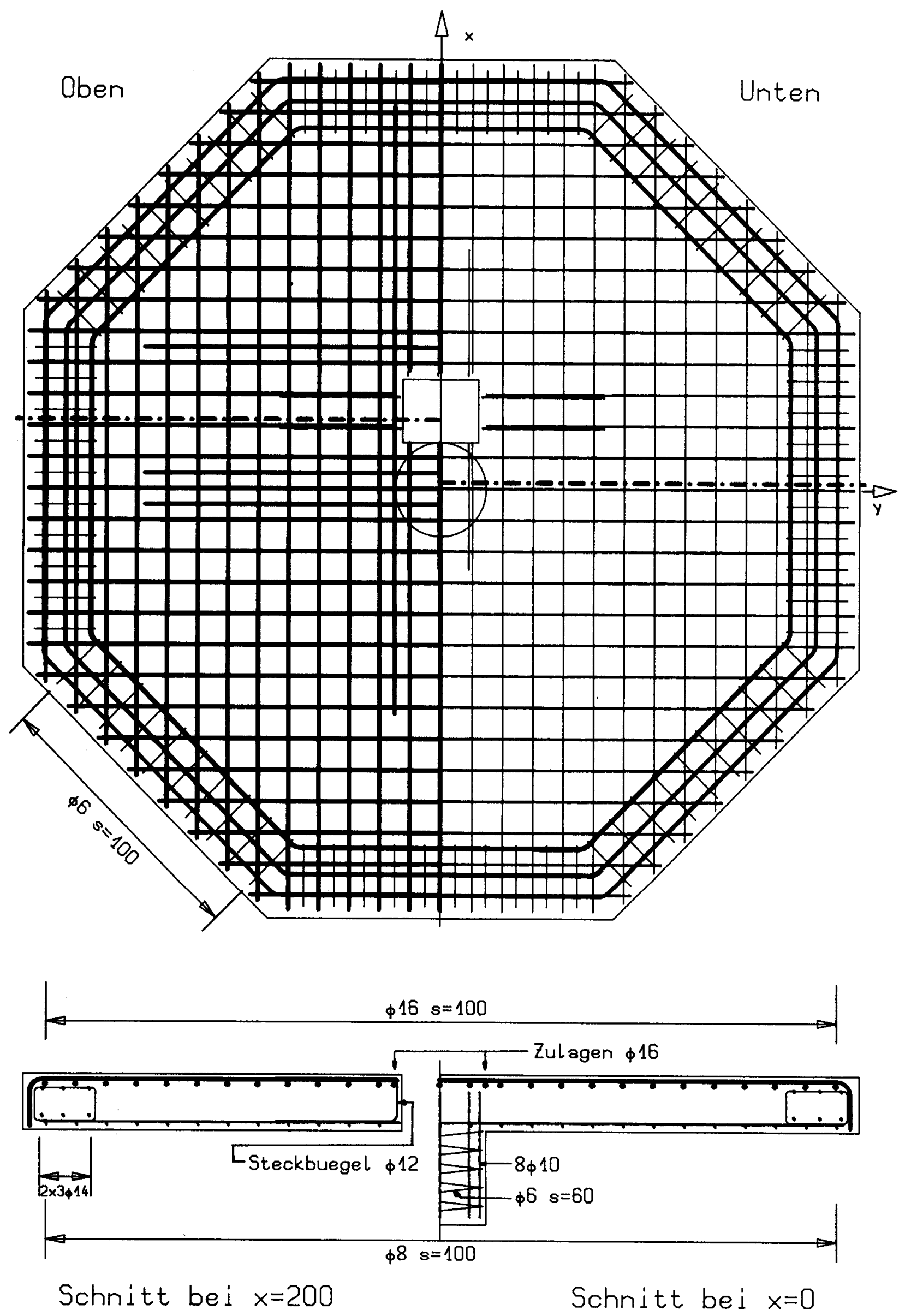

B11d 3: Bewehrungsplan, Platte $P 16$ 

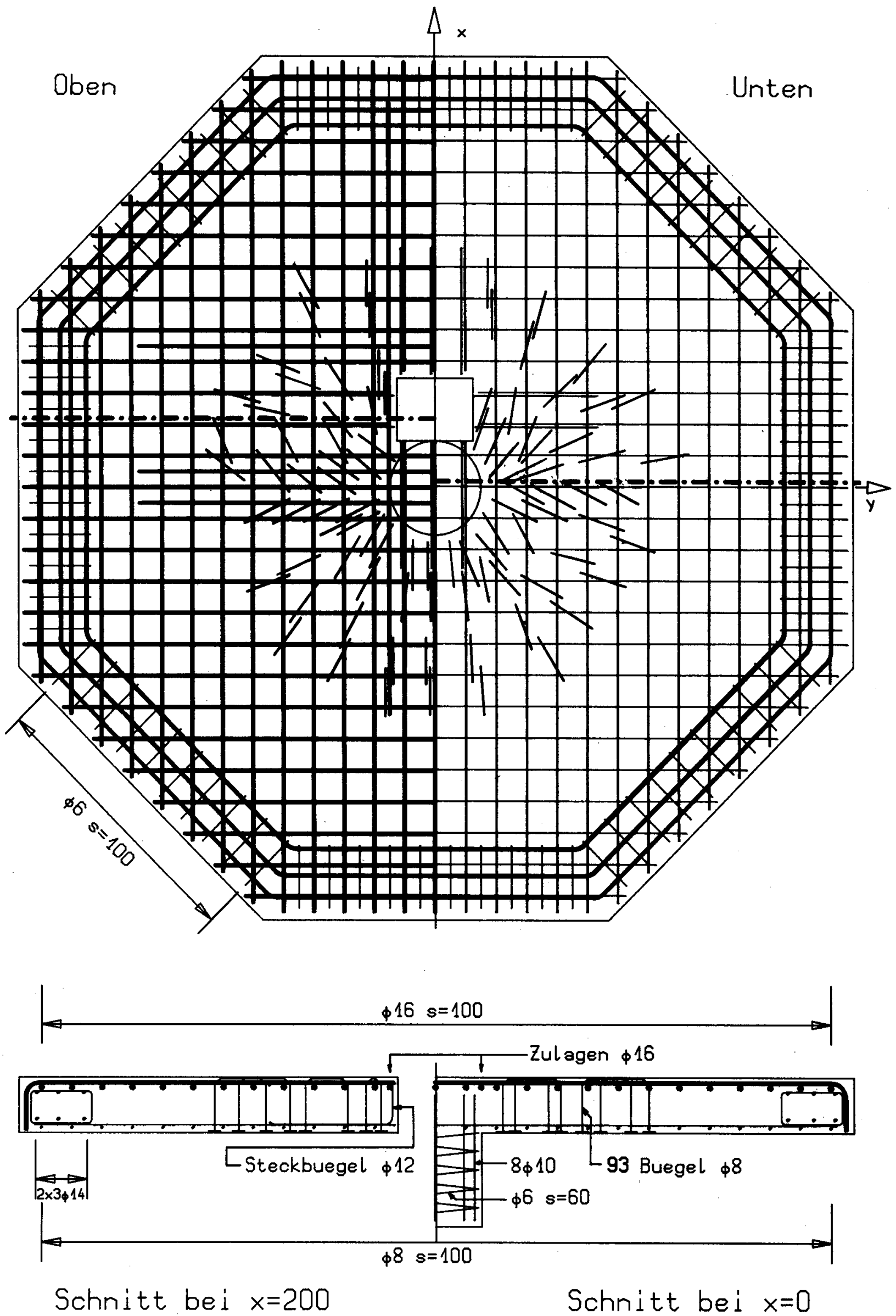

Bild 4: Bewehrungsplan, Platte $P 17$ 

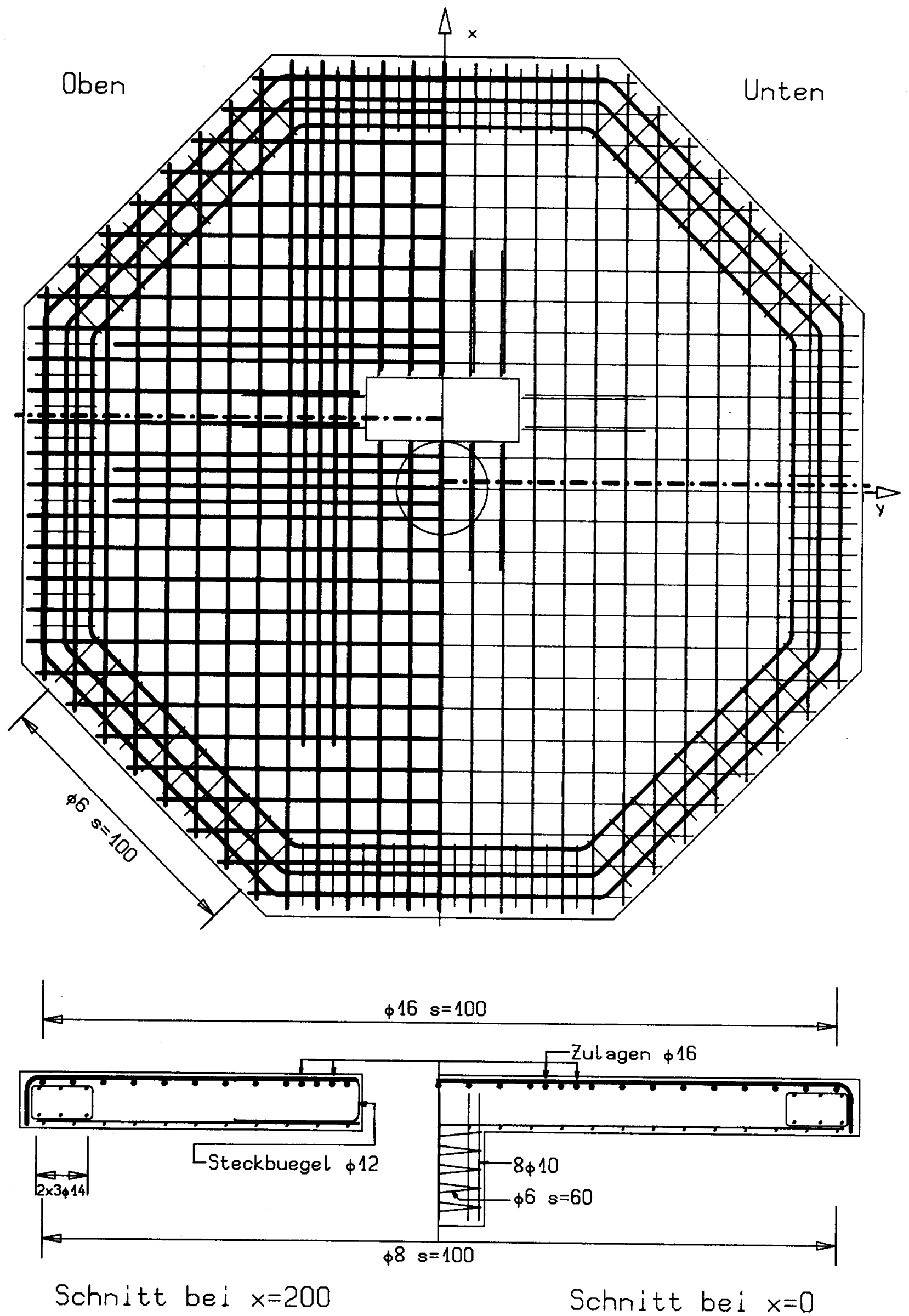

Bild 5: Bewehrungsplan, Platte $P 18$ 

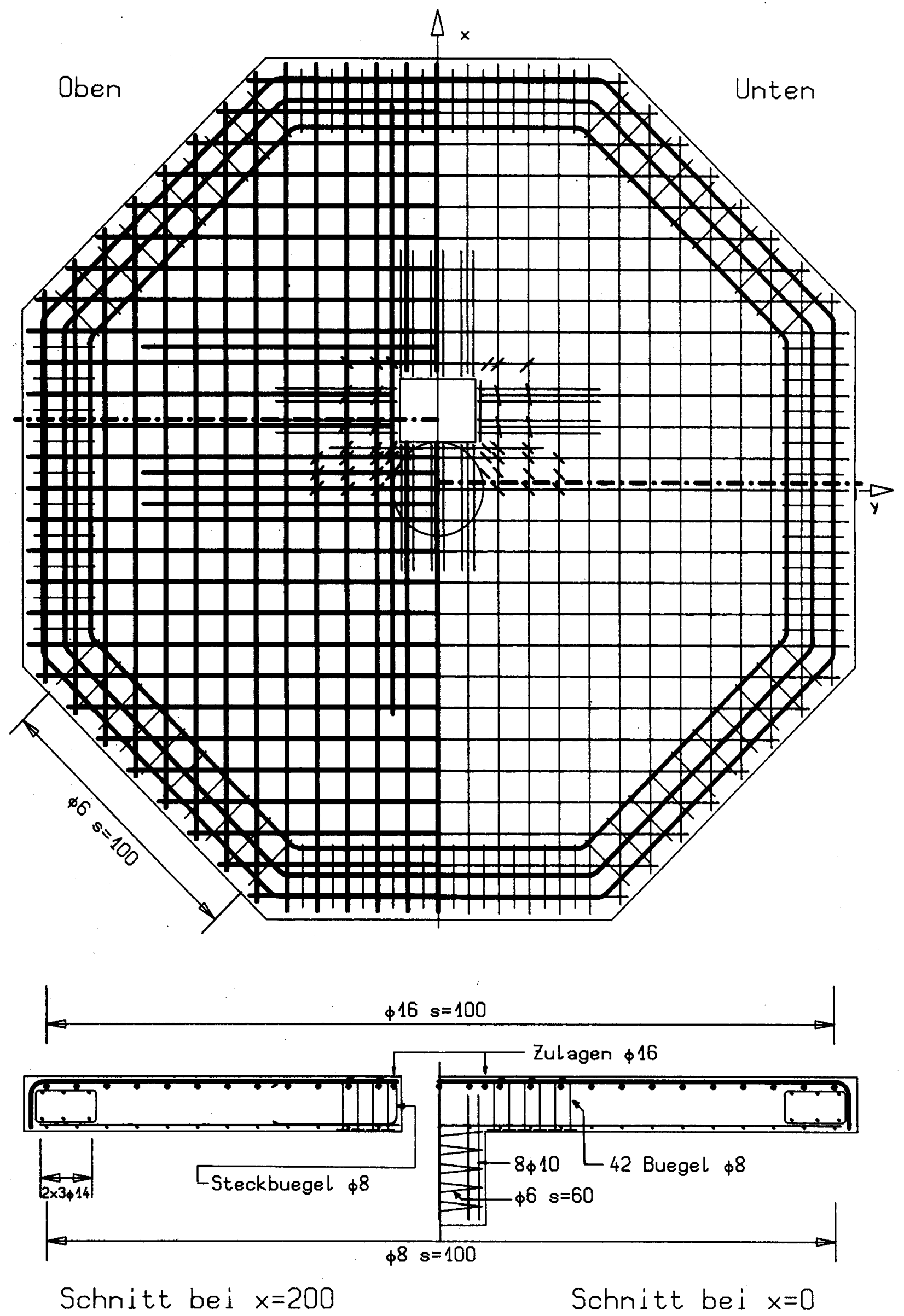

B11d 6: Bewehrungsplan, Platte P 19 

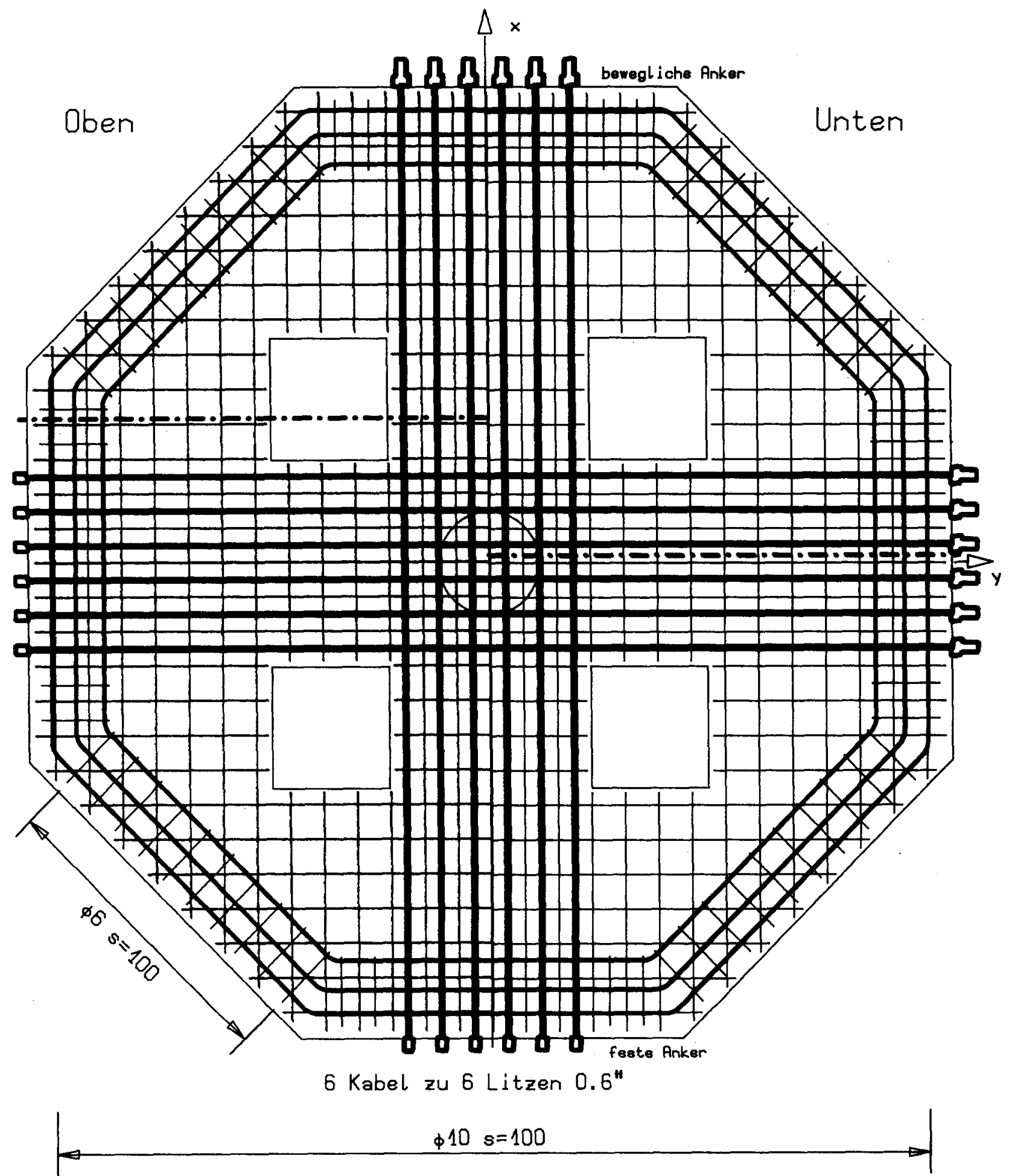

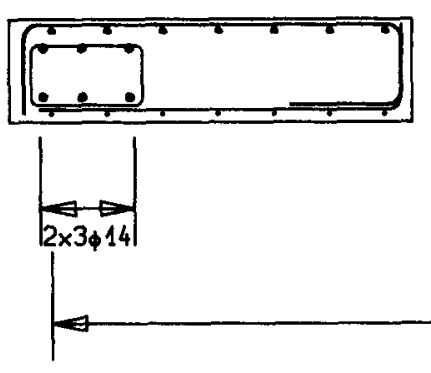

Schnitt be i $x=400$

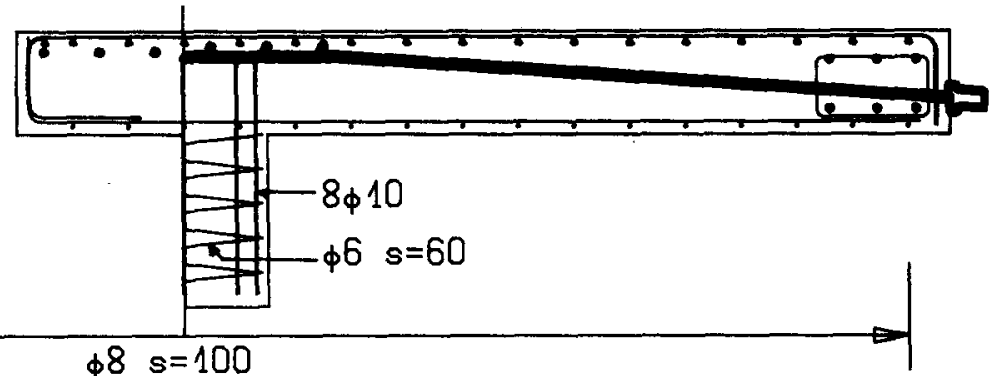

Schnitt bei $x=0$ 


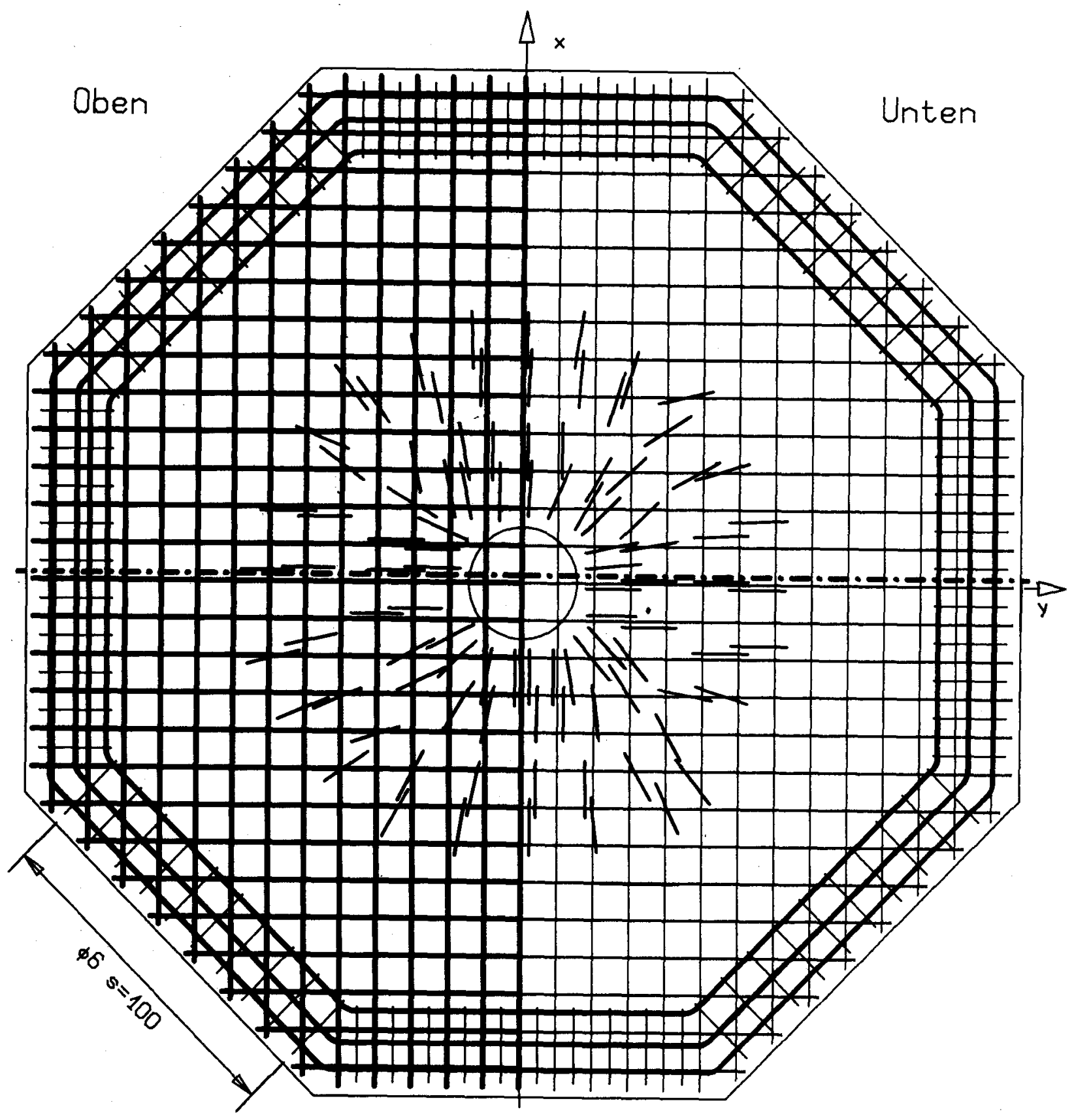

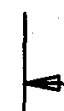
$\phi 16 s=100$

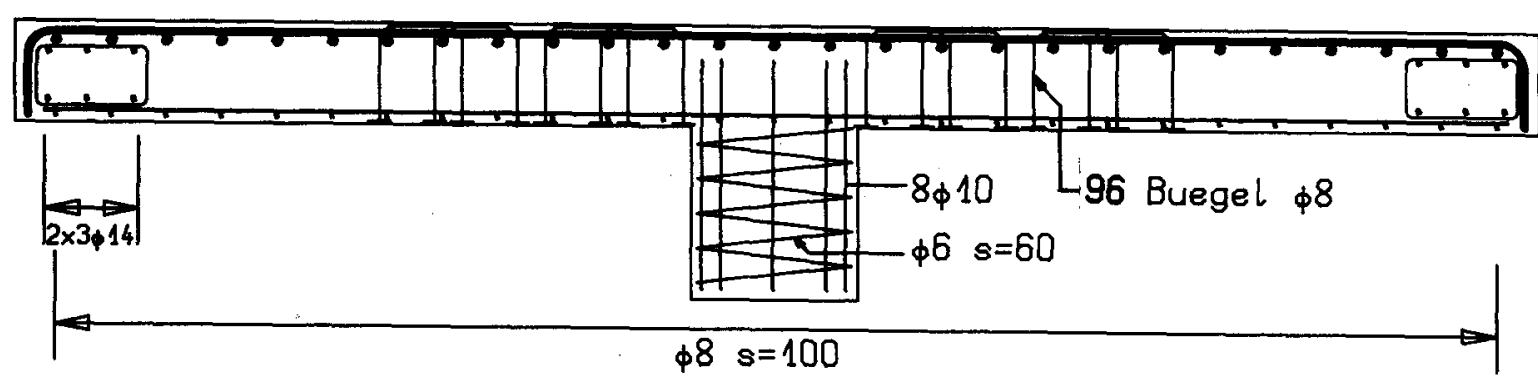

Schnitt be i $x=0$ 


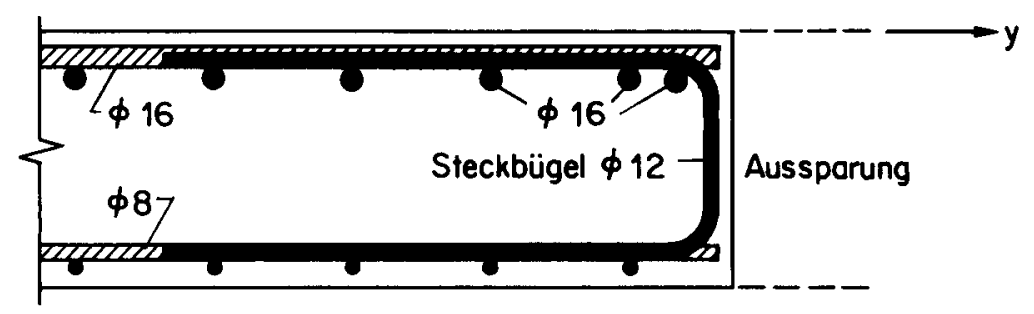

a) Randverbuigelung Platten P16, P17, P18

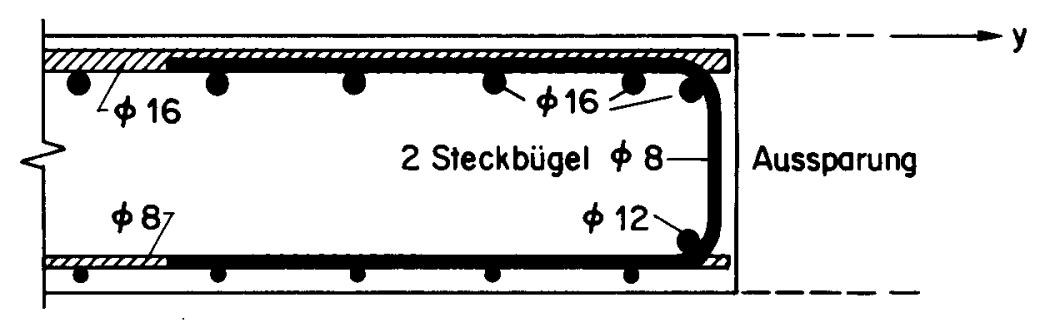

b) Randverbiugelung Platte P 19

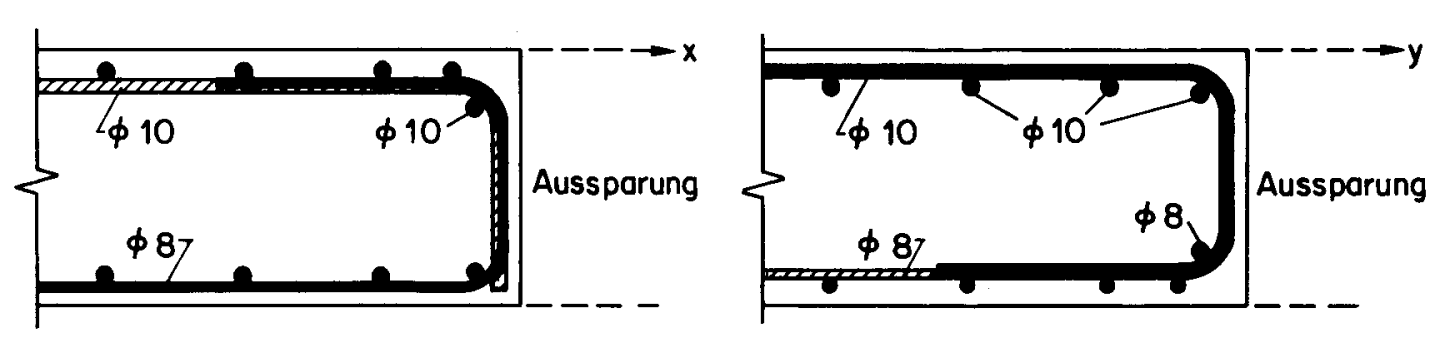

c) Randverbuigelung Platte P2O
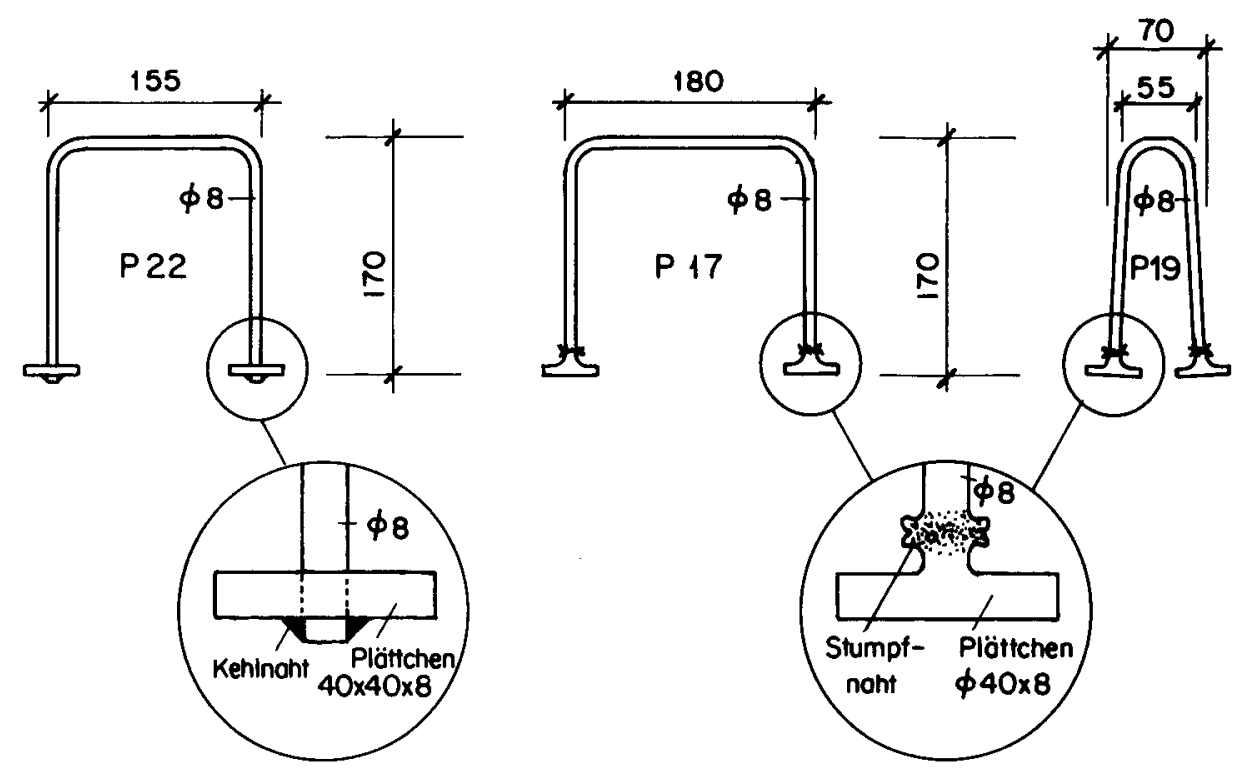

d) Schubbïgel

Bild 9: Bewehrungsdetails 


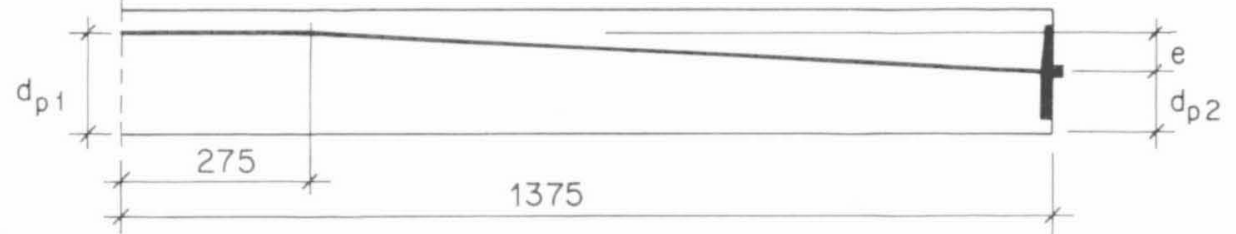

\begin{tabular}{|c|c|c|c|}
\hline & $d_{p 1}$ & $d_{p 2}$ & $e$ \\
\hline & {$[\mathrm{mm}]$} & {$[\mathrm{mm}]$} & {$[\mathrm{mm}]$} \\
\hline Kabel in x-Richtung & 151 & 88 & 63 \\
\hline Kabel in $y$-Richtung & 133 & 70 & 63 \\
\hline
\end{tabular}

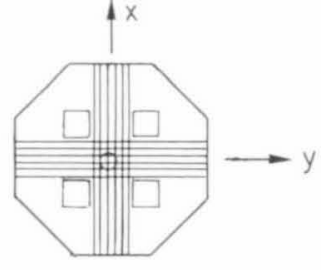

Bild 10: Lage der Spannbewehrung, Platte P 20

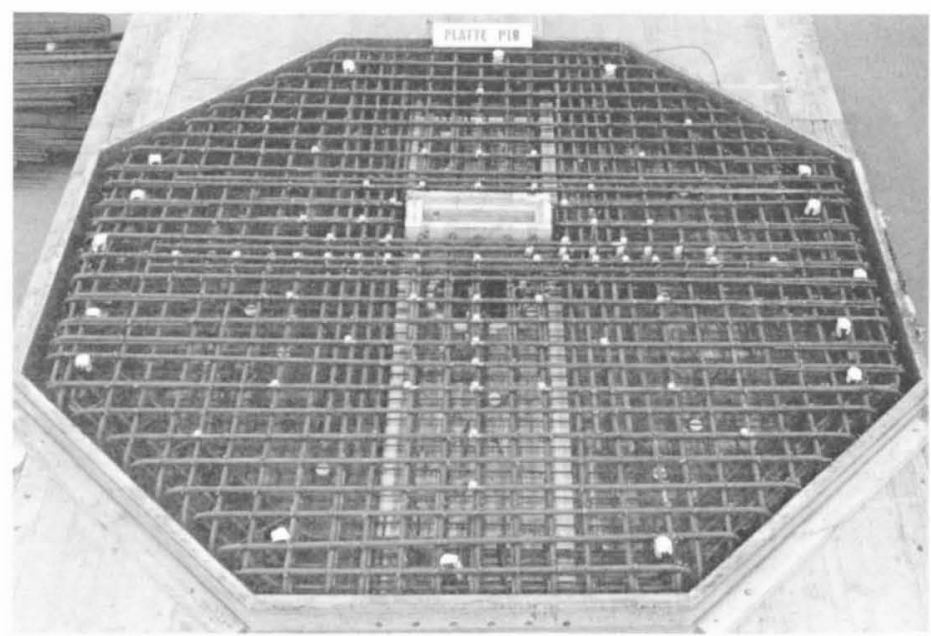

Bild 11: Bewehrung, Platte P 18

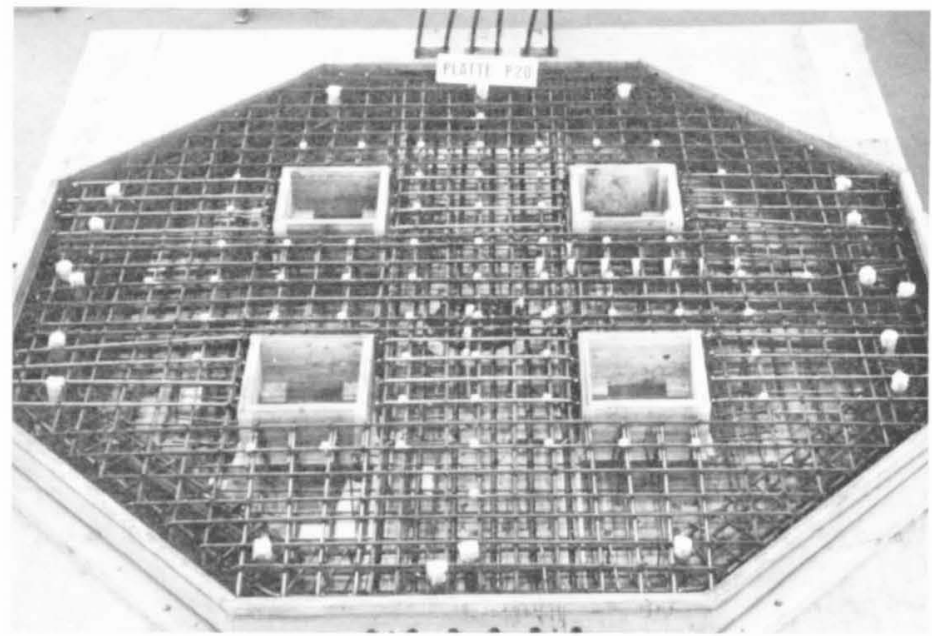

Bild 12: Bewehrung, Platte P 20 

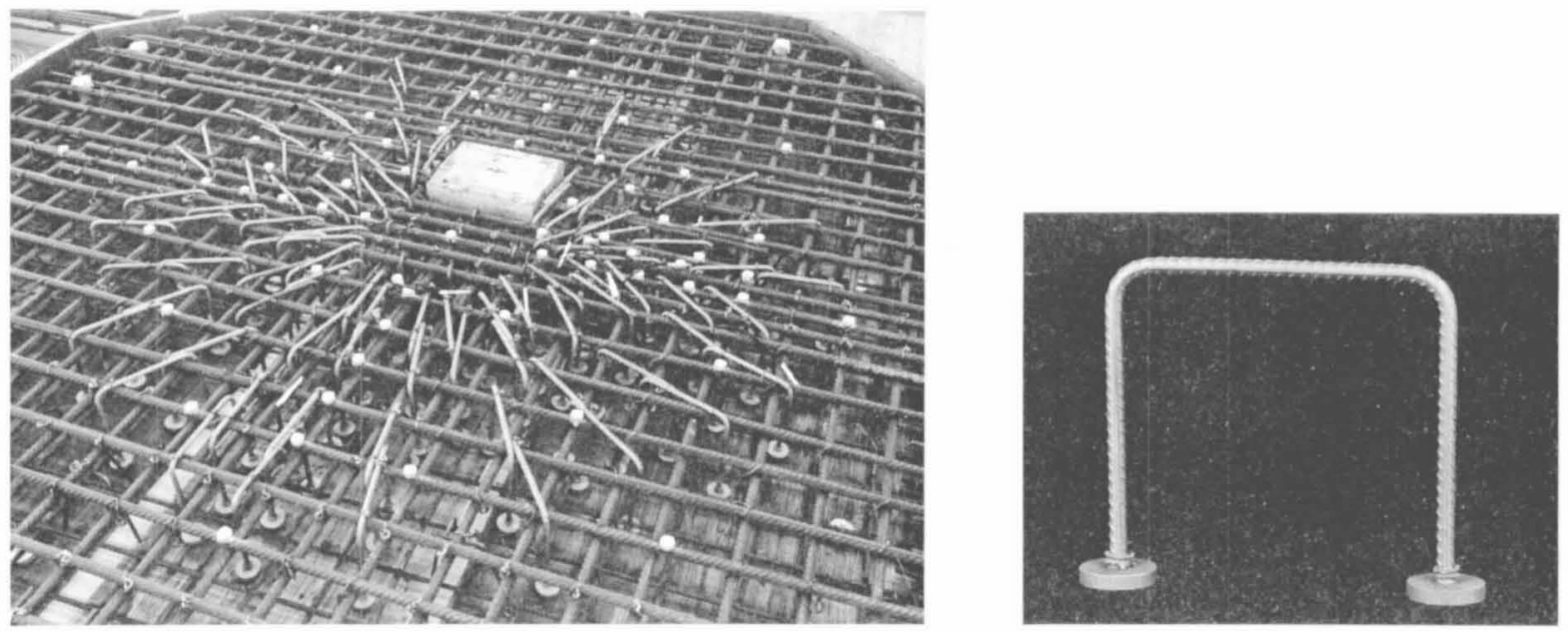

a) Platte P 17
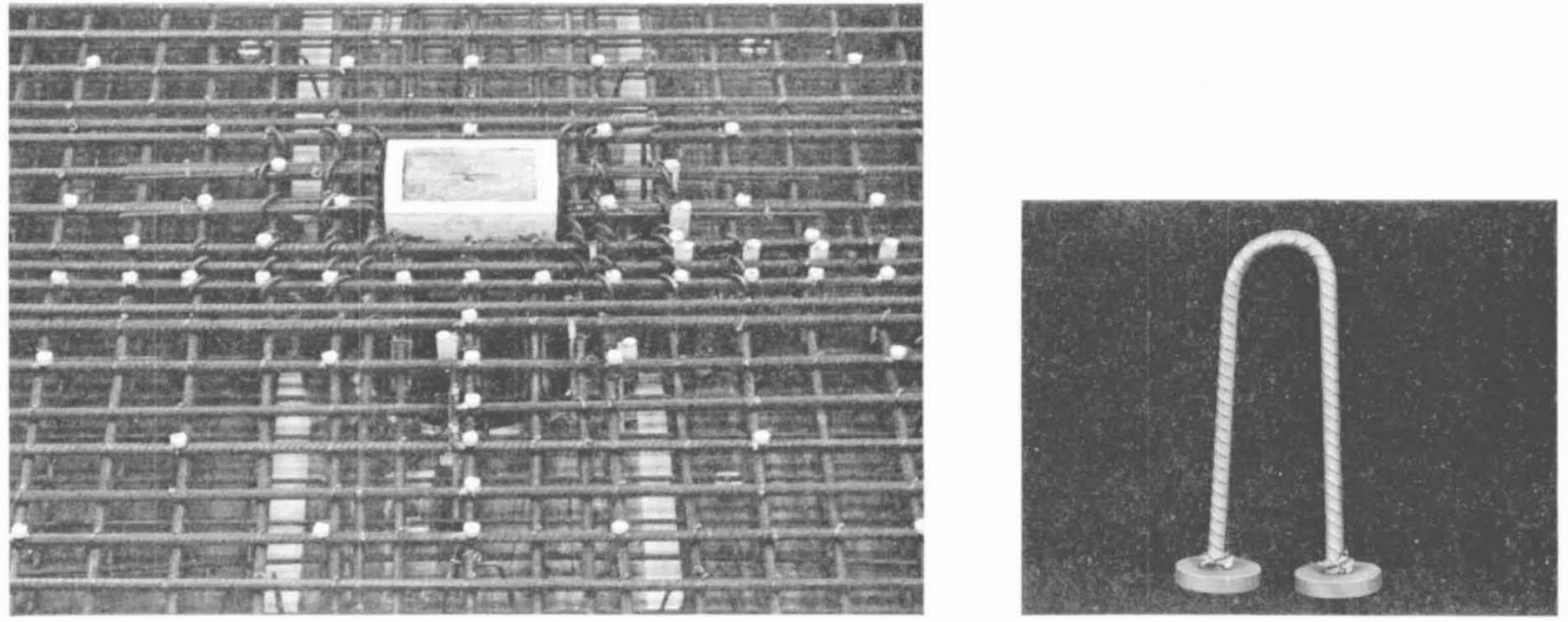

b) Platte P 19
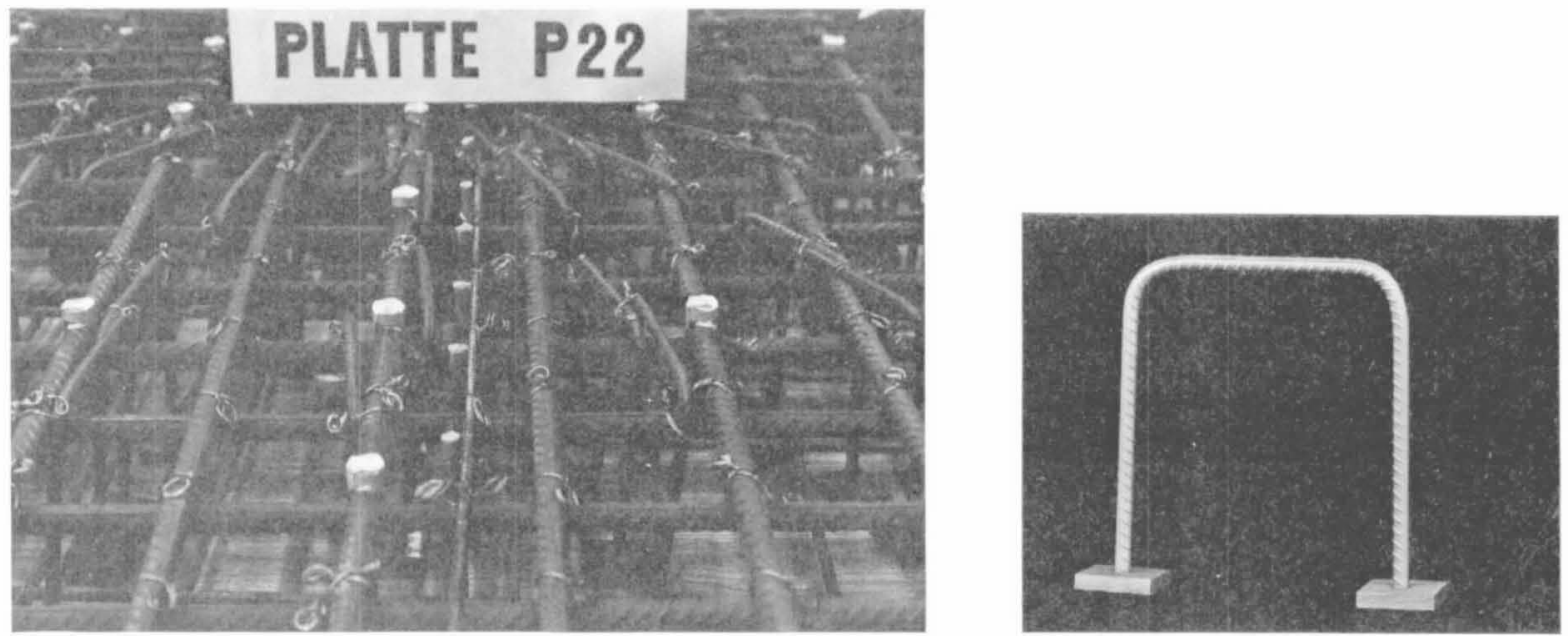

c) Platte P 22

Bild 13: Schubbewehrungen 


\begin{tabular}{|c|c|c|c|c|c|c|c|c|c|}
\hline \multicolumn{4}{|c|}{ Versuch } & $P 16$ & P 17 & $P 18$ & P 19 & $P 20$ & P 22 \\
\hline \multirow{2}{*}{ 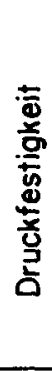 } & Zylinderversuch & $\begin{array}{c}f_{c} \\
\varepsilon_{c u} \\
n\end{array}$ & $\begin{array}{c}{\left[\mathrm{N} / \mathrm{mm}^{2}\right]} \\
{[\% 0]} \\
-\end{array}$ & $\begin{array}{c}29.73 \\
(1.08) \\
2.10 \\
(0.13) \\
6\end{array}$ & $\begin{array}{c}29.55 \\
(1.80) \\
2.35 \\
(0.09) \\
6\end{array}$ & $\begin{array}{c}34.19 \\
(0.70) \\
2.55 \\
(0.10) \\
6\end{array}$ & $\begin{array}{c}33.19 \\
(1.37) \\
252 \\
(0.10) \\
6\end{array}$ & $\begin{array}{c}22.34 \\
(0.83) \\
2.00 \\
(0.27) \\
6\end{array}$ & $\begin{array}{c}32.93 \\
(1.33) \\
2.30 \\
(0.05) \\
5\end{array}$ \\
\hline & Würfelversuch & $\begin{array}{l}f_{c w} \\
n\end{array}$ & $\begin{array}{c}{\left[\mathrm{N} / \mathrm{mm}^{2}\right]} \\
-\end{array}$ & $\begin{array}{c}36.10 \\
(0.89) \\
7\end{array}$ & $\begin{array}{c}33.40 \\
(1.86) \\
7 \\
\end{array}$ & $\begin{array}{c}39.30 \\
(2.33) \\
6 \\
\end{array}$ & $\begin{array}{c}38.99 \\
(1.31) \\
7 \\
\end{array}$ & $\begin{array}{c}28.70 \\
(2.10) \\
7\end{array}$ & $\begin{array}{c}39.50 \\
(2.29) \\
7\end{array}$ \\
\hline \multirow{3}{*}{ 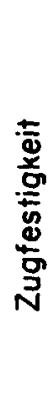 } & Direkter Zugversuch & $\begin{array}{l}f_{c t} \\
n\end{array}$ & $\begin{array}{c}{\left[\mathrm{N} / \mathrm{mm}^{2}\right]} \\
-\end{array}$ & $\begin{array}{c}3.67 \\
(0.09) \\
4 \\
\end{array}$ & $\begin{array}{c}3.62 \\
(0.25) \\
3 \\
\end{array}$ & $\begin{array}{c}3.87 \\
(0.10) \\
3\end{array}$ & $\begin{array}{c}3.16 \\
(0.27) \\
3\end{array}$ & $\begin{array}{c}3.00 \\
(0.28) \\
3\end{array}$ & $\begin{array}{c}3.78 \\
(0.08) \\
4\end{array}$ \\
\hline & Stempelversuch & $\begin{array}{l}f_{c t} \\
n\end{array}$ & $\begin{array}{c}{\left[\mathrm{N} / \mathrm{mm}^{2}\right]} \\
-\end{array}$ & $\begin{array}{c}2.76 \\
(0.41) \\
4 \\
\end{array}$ & $\begin{array}{c}2.79 \\
(0.16) \\
4 \\
\end{array}$ & $\begin{array}{c}3.16 \\
(0.06) \\
4 \\
\end{array}$ & $\begin{array}{c}2.96 \\
0.261 \\
3 \\
\end{array}$ & $\begin{array}{c}2.44 \\
(0.35) \\
4 \\
\end{array}$ & $\begin{array}{c}3.29 \\
(0.25) \\
4\end{array}$ \\
\hline & Brasilianerversuch & $\begin{array}{l}f_{c t} \\
n\end{array}$ & $\begin{array}{c}{\left[\mathrm{N} / \mathrm{mm}^{2}\right]} \\
-\end{array}$ & $\begin{array}{c}2.07 \\
1\end{array}$ & $\begin{array}{c}2.62 \\
1\end{array}$ & $\begin{array}{c}2.31 \\
1\end{array}$ & $\begin{array}{c}2.78 \\
1\end{array}$ & $\begin{array}{c}2.27 \\
1\end{array}$ & $\begin{array}{c}2.65 \\
(0.04) \\
2\end{array}$ \\
\hline
\end{tabular}

Druck-und Zugfestigkeiten

Werte in Klomern: Stondordabweictung

\begin{tabular}{|c|c|c|c|c|c|c|c|c|}
\hline \multicolumn{3}{|l|}{ Versuch } & P 16 & $P 17$ & P 18 & P 19 & P 20 & P 22 \\
\hline \multirow[t]{2}{*}{ Tangentenmodul } & $E_{c 0.5}$ & {$\left[\mathrm{~N} / \mathrm{mm}^{2}\right]$} & $\begin{array}{l}35900 \\
(3900)\end{array}$ & $\begin{array}{l}33600 \\
(2500)\end{array}$ & $\begin{array}{l}33700 . \\
(2500 .)\end{array}$ & $\begin{array}{l}36200 . \\
(5300 .)\end{array}$ & $\begin{array}{l}30100 . \\
(5500)\end{array}$ & $\begin{array}{l}32400 . \\
(1400 .)\end{array}$ \\
\hline & $n$ & - & 6 & 5 & 5 & 4 & 6 & 5 \\
\hline \multirow[t]{2}{*}{ Sekontenmodul } & $E_{c 0.5-10}$ & {$\left[\mathrm{~N} / \mathrm{mm}^{2}\right]$} & $\begin{array}{l}31100 . \\
(1800 .)\end{array}$ & $\begin{array}{c}27800 \\
(900)\end{array}$ & $\begin{array}{l}29500 . \\
(2400 .)\end{array}$ & $\begin{array}{l}30350 . \\
(2500 .)\end{array}$ & $\begin{array}{l}25700 . \\
(5100 .)\end{array}$ & $\begin{array}{l}31600 . \\
(1300 .)\end{array}$ \\
\hline & $n$ & - & 6 & 5 & 5 & 4 & 6 & 5 \\
\hline
\end{tabular}

E-Moduli

Werte in Klamern: Standardabweicthung

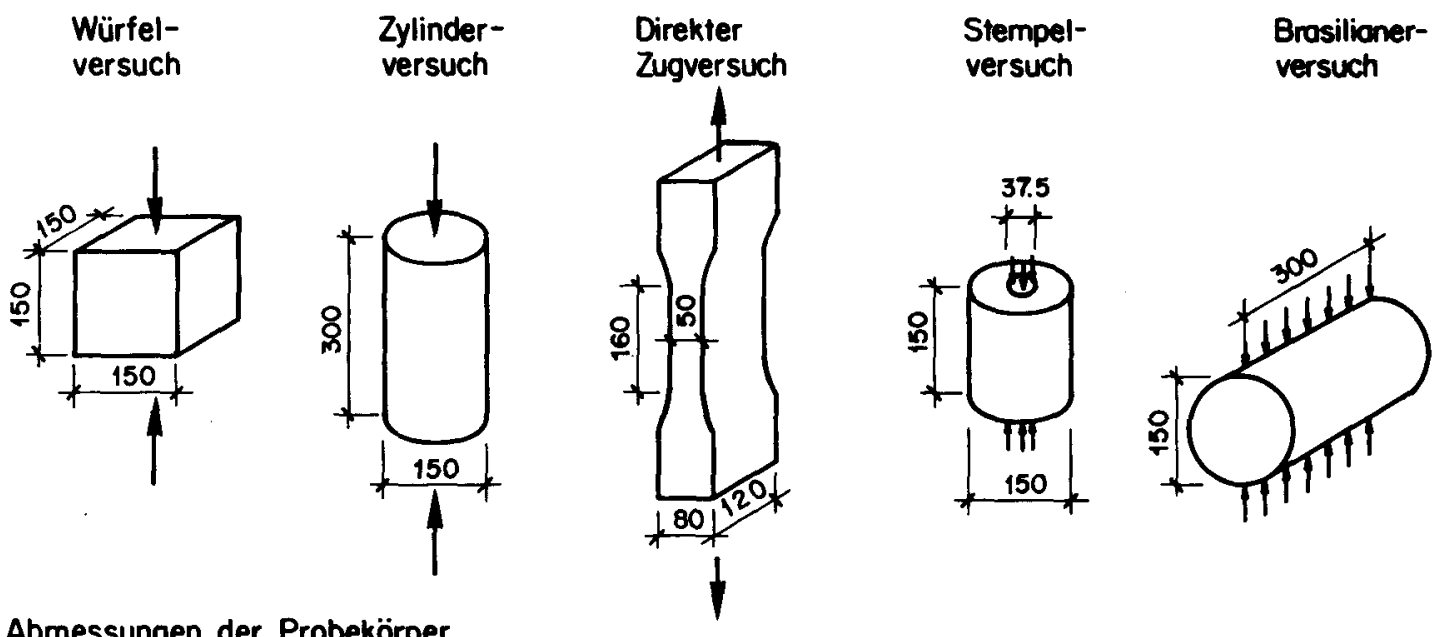

Abmessungen der Probekörper 


\begin{tabular}{|c|c|c|c|c|c|c|c|c|c|c|c|}
\hline Stohltyo & $\begin{array}{c}\phi \\
{[\mathrm{mm}]}\end{array}$ & $n$ & Verwendung & $\begin{array}{l}A_{\text {eff }} \\
{\left[\mathrm{mm}^{2}\right]}\end{array}$ & $\begin{array}{c}t_{\mathrm{sy}, \text { stot }} \\
{\left[\mathrm{N} / \mathrm{mm}^{2}\right]}\end{array}$ & $\begin{array}{c}f_{y y, d y n} \\
{\left[\mathrm{~N} / \mathrm{mm}^{2}\right]}\end{array}$ & {$\left[\begin{array}{c}f_{s t} \\
{\left[N / \mathrm{mm}^{2}\right]}\end{array}\right.$} & $\frac{t_{s t}}{t_{s y, d y n}}$ & {$\left[\begin{array}{c}E_{s} \\
{\left[k N / m^{2}\right]}\end{array}\right.$} & $\begin{array}{l}\varepsilon_{\text {sh }} \\
{[\% 0]}\end{array}$ & $\begin{array}{c}8 g 1 \\
{[\% \circ]}\end{array}$ \\
\hline Topor & 8. & 8 & $P\{6-19,22$ & $\begin{array}{c}50.46 \\
(.63)\end{array}$ & $\begin{array}{l}562.9 \\
(17.0)\end{array}$ & $\begin{array}{l}586.4 \\
(15.5)\end{array}$ & $\begin{array}{l}643.4 \\
(15.5)\end{array}$ & $\begin{array}{c}1.14 \\
(0.01)\end{array}$ & $\begin{array}{r}200.3 \\
(.8)\end{array}$ & $\begin{array}{l}29.3 \\
(2.4)\end{array}$ & $\begin{array}{l}74.2 \\
(7.2)\end{array}$ \\
\hline Topor & 10. & 4 & P20 & $\begin{array}{l}77.22 \\
(.09)\end{array}$ & $\begin{array}{l}557.2 \\
(13.2)\end{array}$ & $\begin{array}{l}586.0 \\
(5.5)\end{array}$ & $\begin{array}{c}654.0 \\
(6.7)\end{array}$ & $\begin{array}{c}1.17 \\
(0.02)\end{array}$ & $\begin{array}{c}197.6 \\
1.71\end{array}$ & $\begin{array}{l}23.6 \\
(\quad .8)\end{array}$ & $\begin{array}{l}71.1 \\
(5.4)\end{array}$ \\
\hline Topor & 12. & 4 & P16. 18 & $\begin{array}{c}111.20 \\
(1.76)\end{array}$ & $\begin{array}{c}540.0 \\
(7.8)\end{array}$ & $\mid \begin{array}{l}558.4 \\
(7.5)\end{array}$ & $\begin{array}{l}641.0 \\
(15.1)\end{array}$ & $\begin{array}{c}1.19 \\
(0.01)\end{array}$ & $\begin{array}{r}196.1 \\
(2.0)\end{array}$ & 13.9 & $\begin{array}{r}96.5 \\
(4.2)\end{array}$ \\
\hline Topor & 14. & 4 & $P 16-20,22$ & $\begin{array}{c}153.52 \\
(.13)\end{array}$ & $\begin{array}{l}518.6 \\
(11.6)\end{array}$ & $\begin{array}{l}540.5 \\
(12.4)\end{array}$ & $\begin{array}{c}625.4 \\
(8.1)\end{array}$ & $\begin{array}{c}1.21 \\
(0.02)\end{array}$ & $\begin{array}{c}196.6 \\
(3.6)\end{array}$ & $\begin{array}{l}19.9 \\
(2.3)\end{array}$ & $\begin{array}{l}82.8 \\
(9.6)\end{array}$ \\
\hline Topor & 16. & 4 & $P 16-18,22$ & $\begin{array}{r}203.93 \\
1.041\end{array}$ & $\begin{array}{l}550.7 \\
(3.8)\end{array}$ & $\begin{array}{l}575.7 \\
14.01\end{array}$ & $\begin{array}{l}670.3 \\
(3.7)\end{array}$ & $\begin{array}{c}1.22 \\
(0.01)\end{array}$ & $\begin{array}{c}196.2 \\
(1.8)\end{array}$ & $\begin{array}{l}14.9 \\
1.41\end{array}$ & $\begin{array}{c}106.6 \\
(7.9)\end{array}$ \\
\hline Topor & 16. & 4 & $P 19$ & $\begin{array}{c}193.72 \\
(1.14)\end{array}$ & $\begin{array}{l}507.3 \\
(21.0)\end{array}$ & $\begin{array}{l}534.1 \\
(16.0)\end{array}$ & $\begin{array}{l}637.3 \\
(14.2)\end{array}$ & $\begin{array}{c}1.26 \\
(0.02)\end{array}$ & $\begin{array}{c}199.0 \\
(2.7)\end{array}$ & $\left(\begin{array}{c}15.4 \\
(.9)\end{array}\right.$ & $\begin{array}{c}96.4 \\
(14.7)\end{array}$ \\
\hline Tor ip & 8. & 4 & P2O & $\begin{array}{l}51.14 \\
(.11)\end{array}$ & $\begin{array}{l}498.0 * \\
15.01\end{array}$ & $\begin{array}{l}518.9 * \\
(4.0)\end{array}$ & $\begin{array}{l}608.1 \\
(2.6)\end{array}$ & $\begin{array}{l}1.22 \\
(0.01)\end{array}$ & $\begin{array}{r}198.7 \\
(.91\end{array}$ & - & $\begin{array}{l}26.1 \\
(5.0)\end{array}$ \\
\hline
\end{tabular}

* Streckgrenze (bei $0.2 \%$ bleibender Dehnung)

Werte in Klommern: Stondordobweichung

a) Kennwerte der Biegebewehrung

\begin{tabular}{|c|c|c|c|c|c|c|c|}
\hline Stahtyp & $\phi$ & $n$ & verwendung & $F_{y, \text { stat }}$ & $F_{y, d y n}$ & $F_{f}$ & $\frac{F_{f}}{F_{y_{1}} \text { stat }}$ \\
\hline Topor & 8 & 4 & $P 17$ & 28.62 & 29.57 & 32.95 & 1.151 \\
& & & & $(0.67)$ & $(0.68)$ & $(0.81)$ & $(0.004)$ \\
Topor & 8 & 4 & $P 19$ & 26.65 & 27.72 & 34.12 & 1.168 \\
& & & & $(0.931$ & $(0.97)$ & $(0.04)$ & $(0.009)$ \\
Topor & 8 & 4 & $P 22$ & 28.40 & 29.59 & 32.47 & 1.143 \\
& & & & $(0.93)$ & $(0.86)$ & $(0.88)$ & $(0.049)$ \\
\hline
\end{tabular}

Probestob

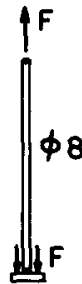

Werte in Klommern: Standardobweichung

Ankerpläitchen

b) Kennwerte der Schubbewehrung

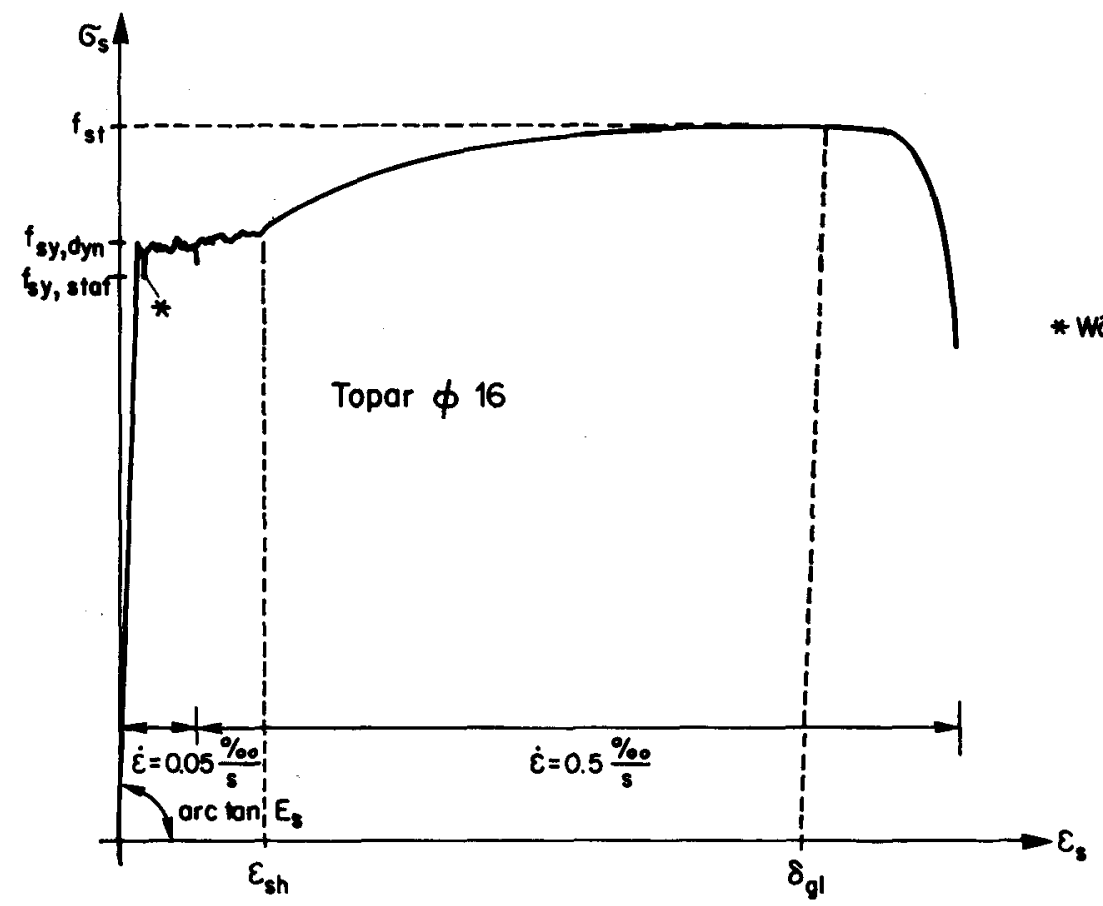

c) Sponnungs-Dehnungsdiagramm eines Bewehrungsstahls

B1ld 15: Kennwerte des Bewehrungsstahls 

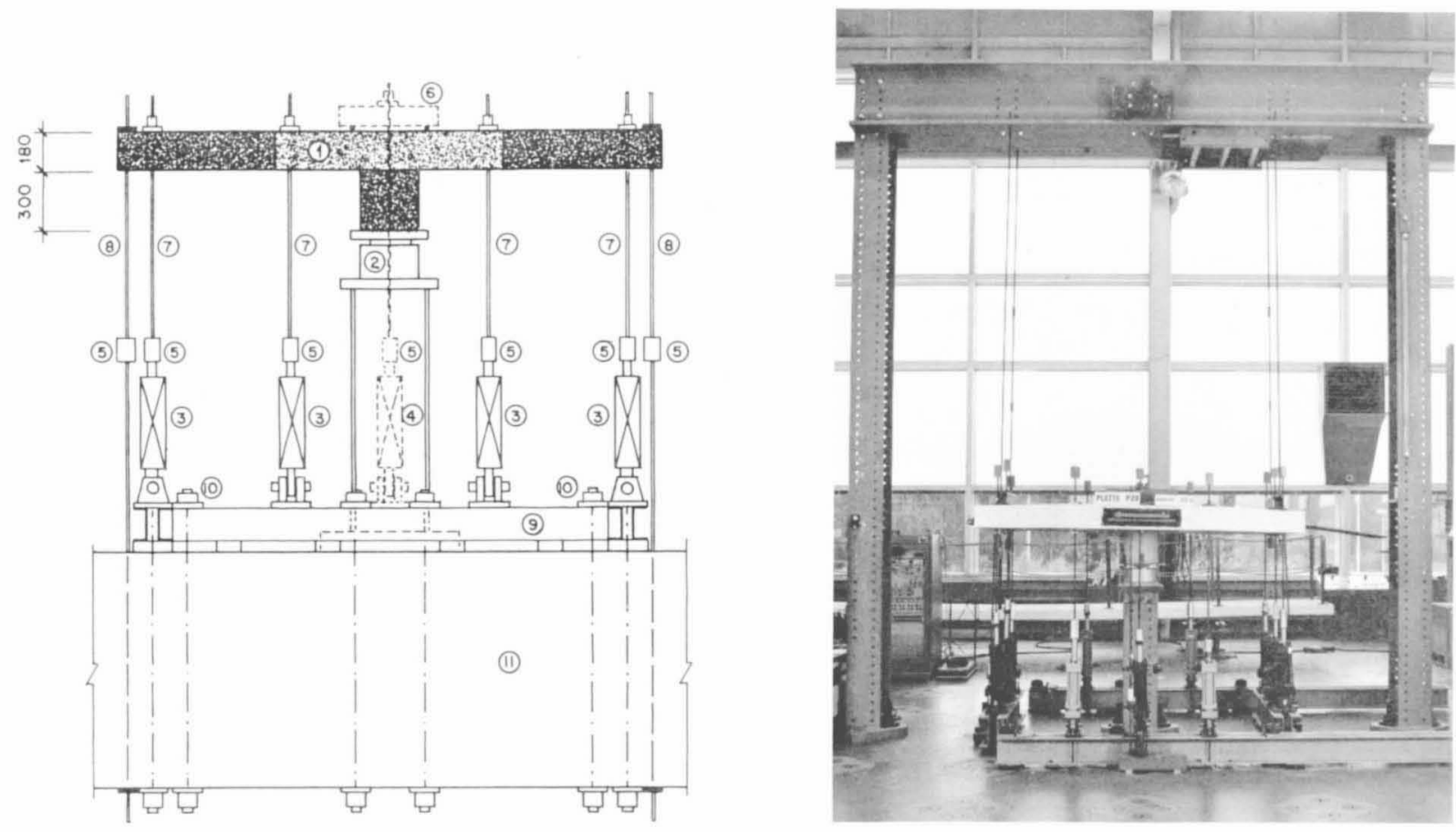

Gesamtansicht der Versuchsanlage mit Sicherungsrahmen für Platte P 20

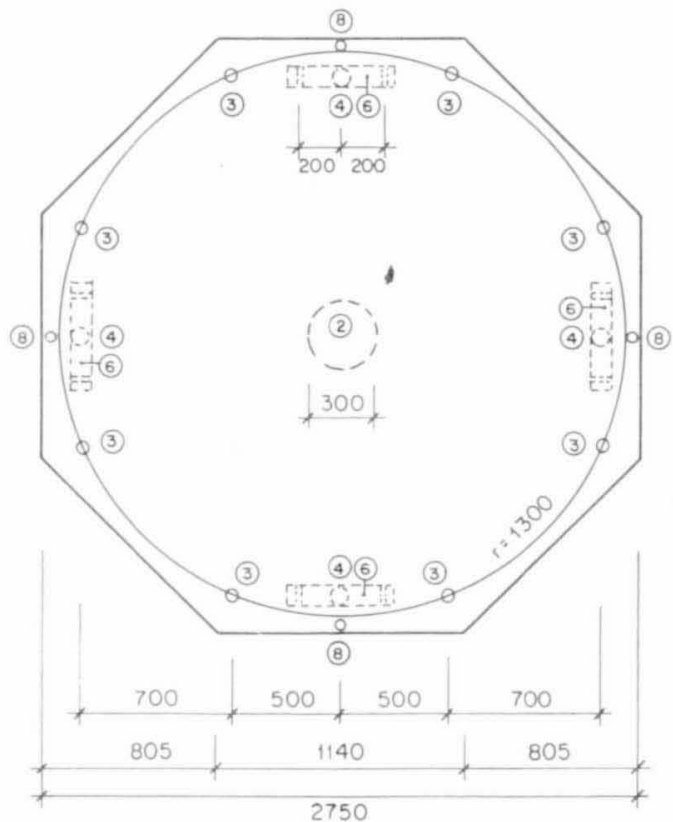

(1) Versuchskörper

(2) Flochpresse $2000 \mathrm{kN}$ zur Messung der Auflogerreoktion

(3) 8 Zugkolben

(4) 4 zusäłzliche Zugkolben ( $P 20$ und P22)

(5) Kroftmessdosen $150 \mathrm{kN}$

(6) Lostverteilbolken

(7) Zugstangen

(B) Stabilisierungsstangen

(9) Montogetröger für Zugkolben

(10) Veronkerung Montagetröger

(11) Aufsponnboden 

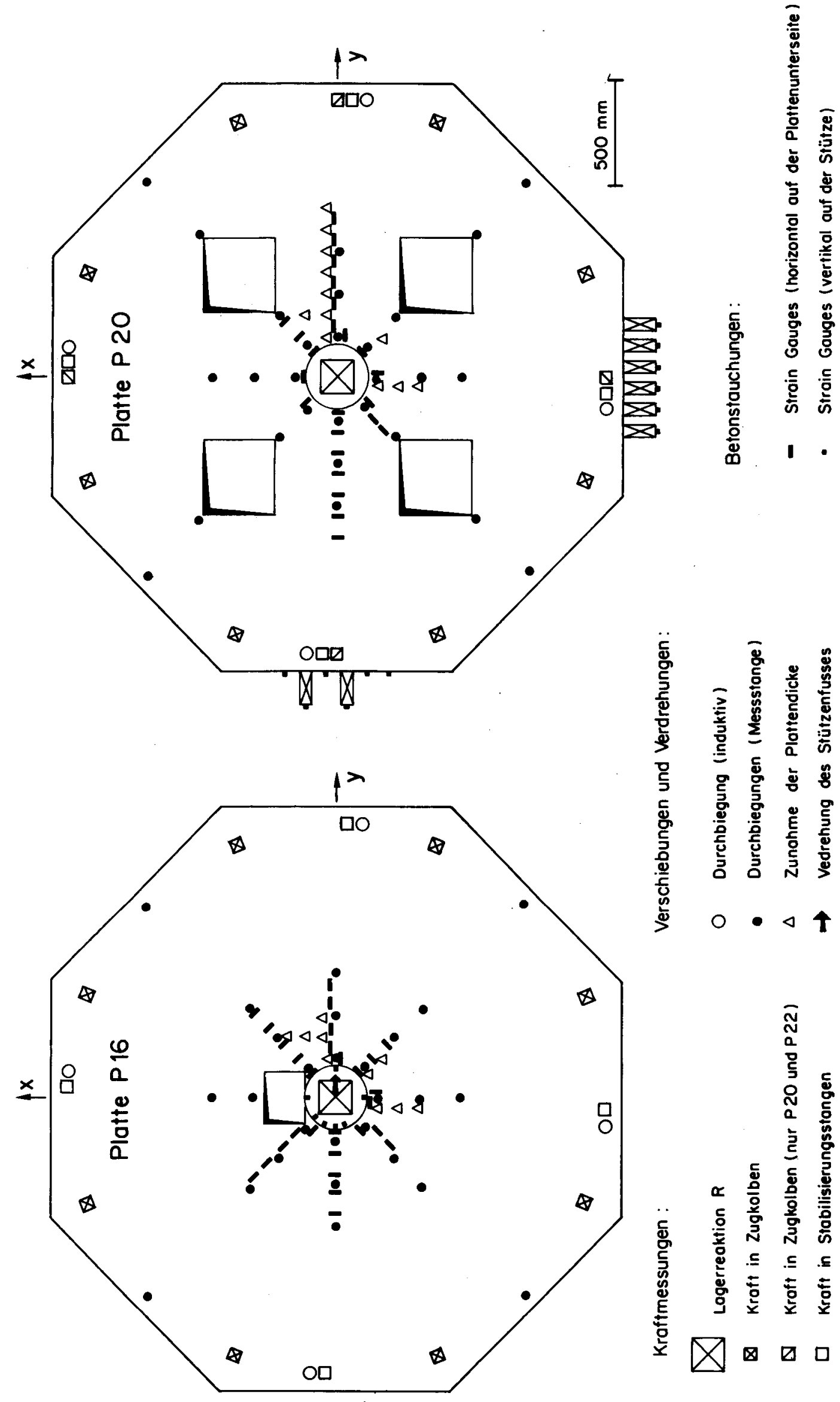

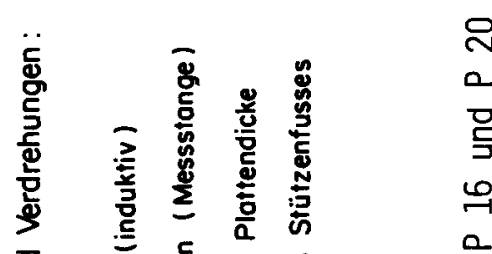

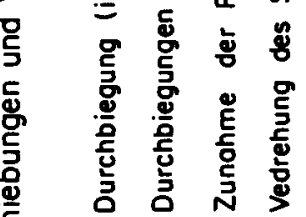

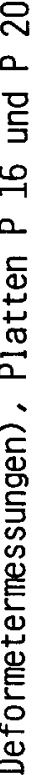

N

ำ

옹

$\triangleleft 4$

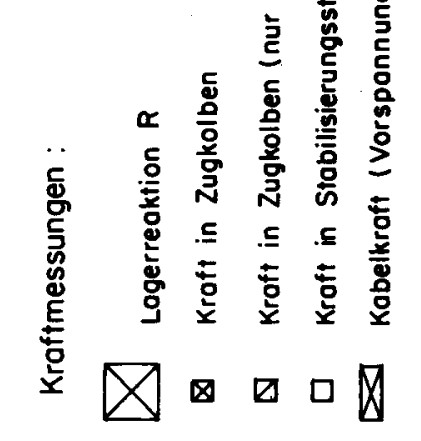

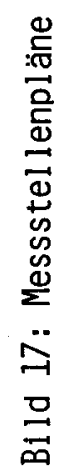




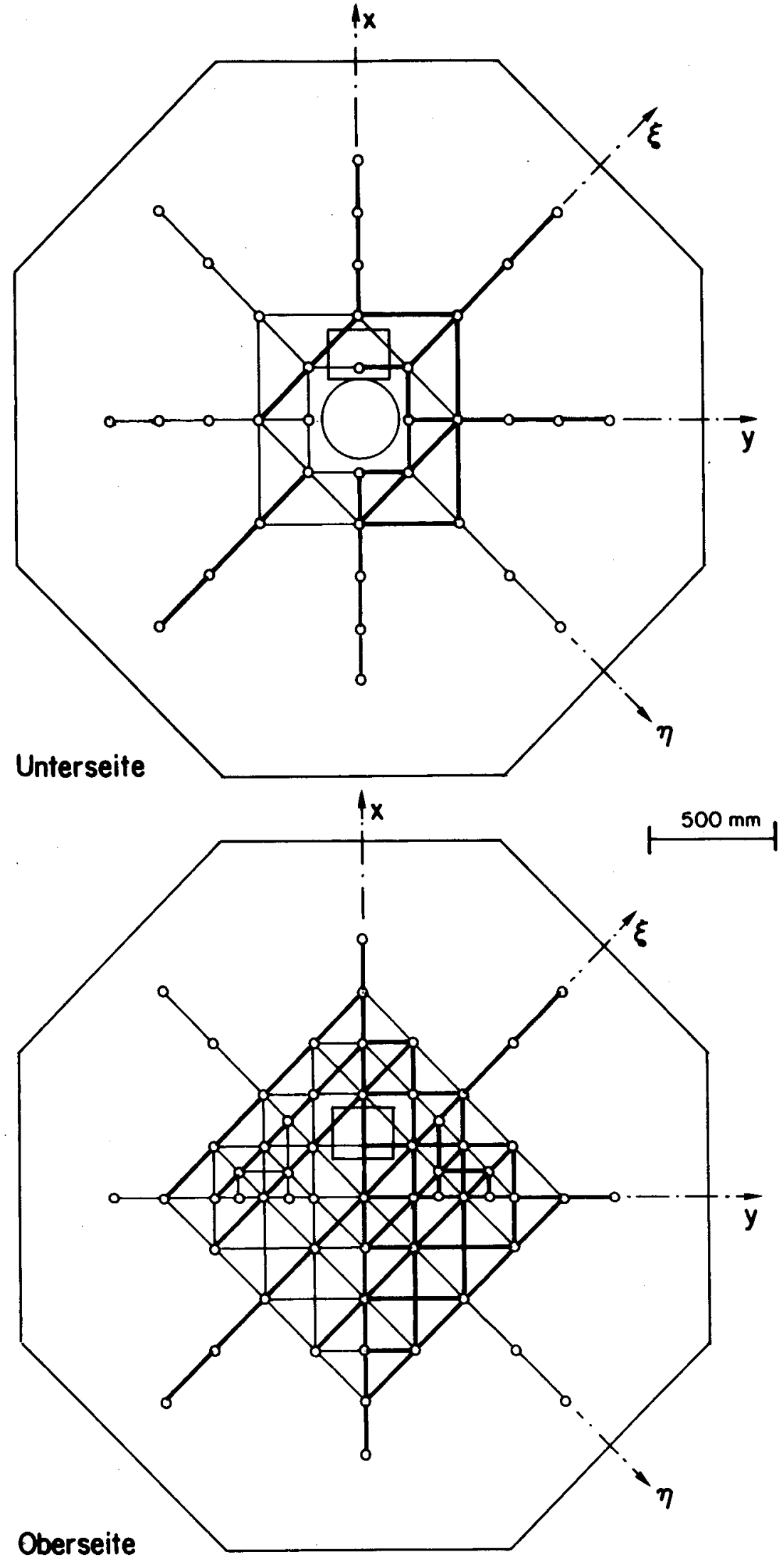

$\multimap$ in Tabelle $A 1$ nicht dorgestellte Dehnungen

$\multimap$ in Tabelle A1 dargestellte Dehnungen

Bild 18: Mess- und Auswertungsnetze, Platte P 16 


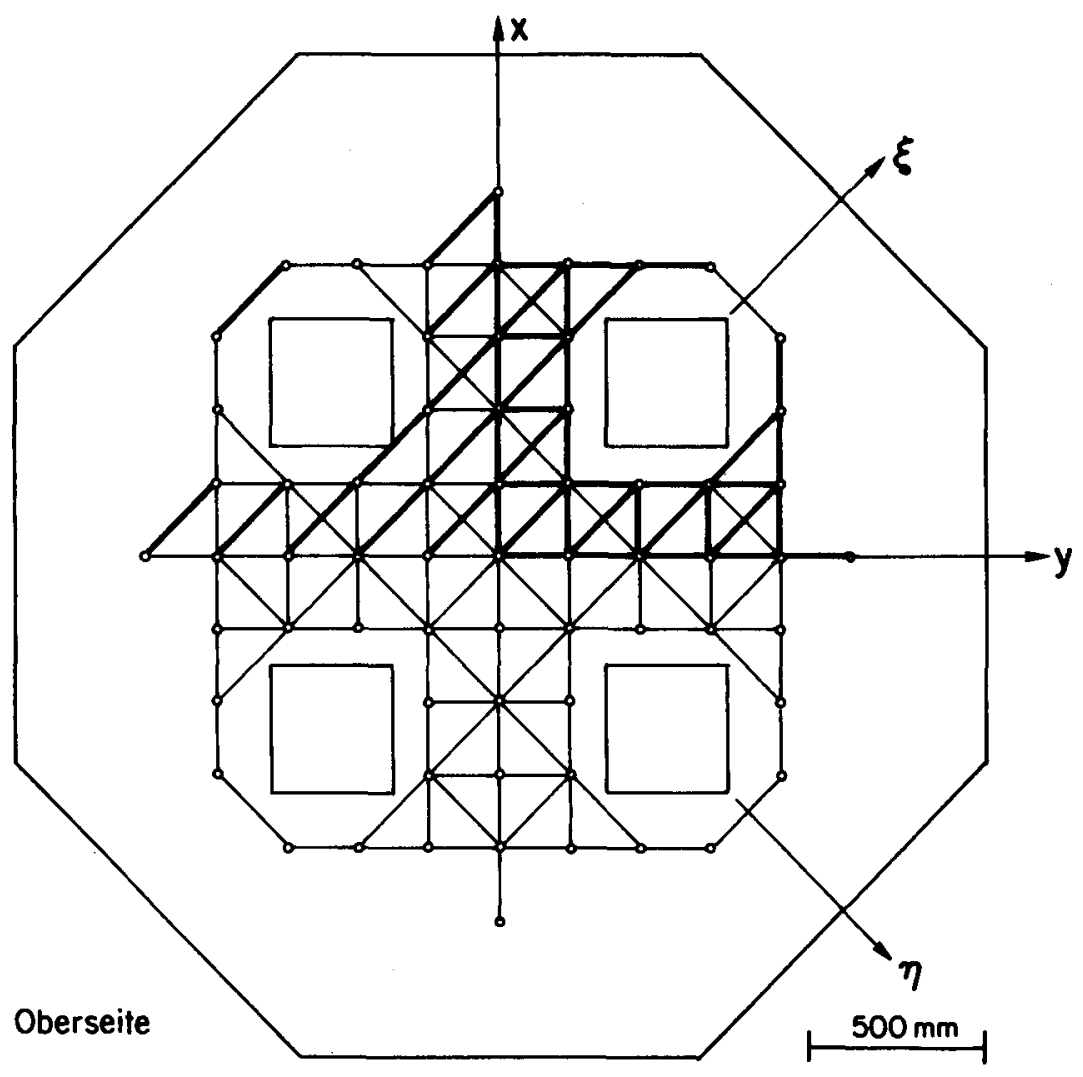

$\leadsto$ in Tabelle A5 nicht dargestellte Dehnungen

$\longrightarrow$ in Tabelle A5 dargestellte Dehnungen

Bemerkung : Mess-und Auswertungsnetz Unterseite onolog, aber bei der Stütze unterbrochen

Bild 19: Mess- und Auswertungsnetz, Platte P 20, Oberselte

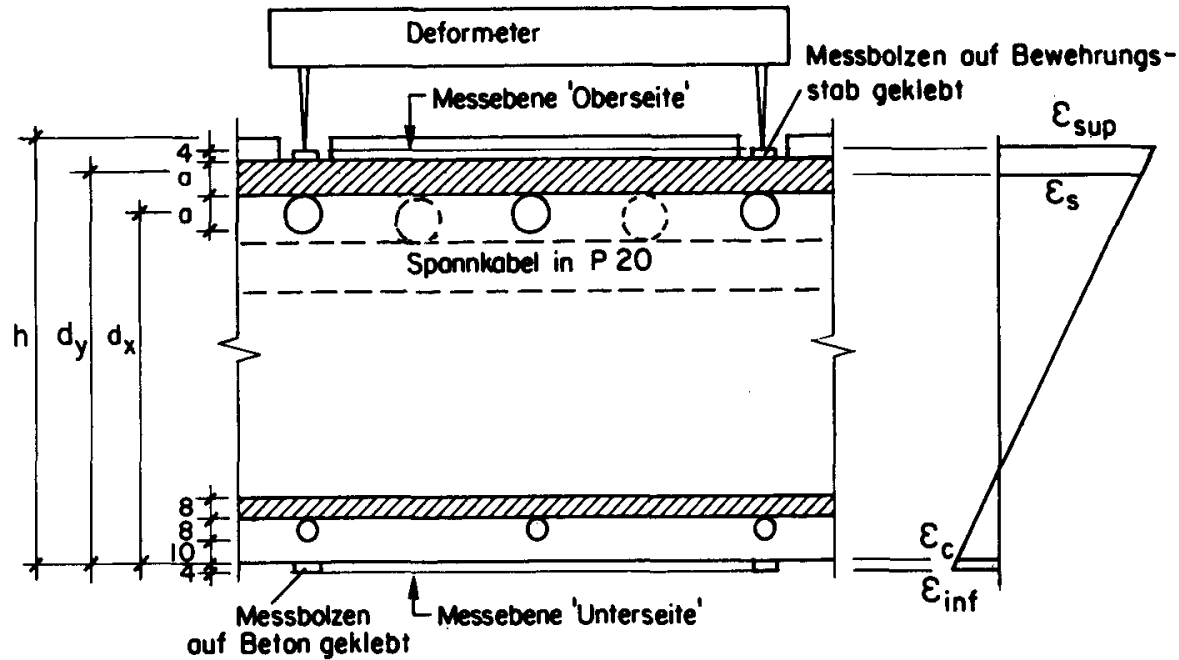

\begin{tabular}{|lll|r|r|r|r|r|r|}
\hline & & & $P 16$ & $P 17$ & $P 18$ & $P 19$ & $P 20$ & $P 22$ \\
\hline Mittlere Dicke & $h$ & {$[\mathrm{~mm}]$} & 185 & 184 & 185 & 185 & 185 & 185 \\
\hline Stabdurchmesser & $o$ & {$[\mathrm{~mm}]$} & 16 & 16 & 16 & 16 & 10 & 16 \\
\hline Statische Höhe & $d_{x}$ & {$[\mathrm{~mm}]$} & 144 & 145 & 145 & 145 & 155 & 145 \\
\hline Statische Höhe & $d_{y}$ & {$[\mathrm{~mm}]$} & 160 & 162 & 162 & 162 & 165 & 162 \\
\hline
\end{tabular}

Bild 20: Gemessene mittlere Dicken der Platten, statische Höhen und Lage der Messebenen 


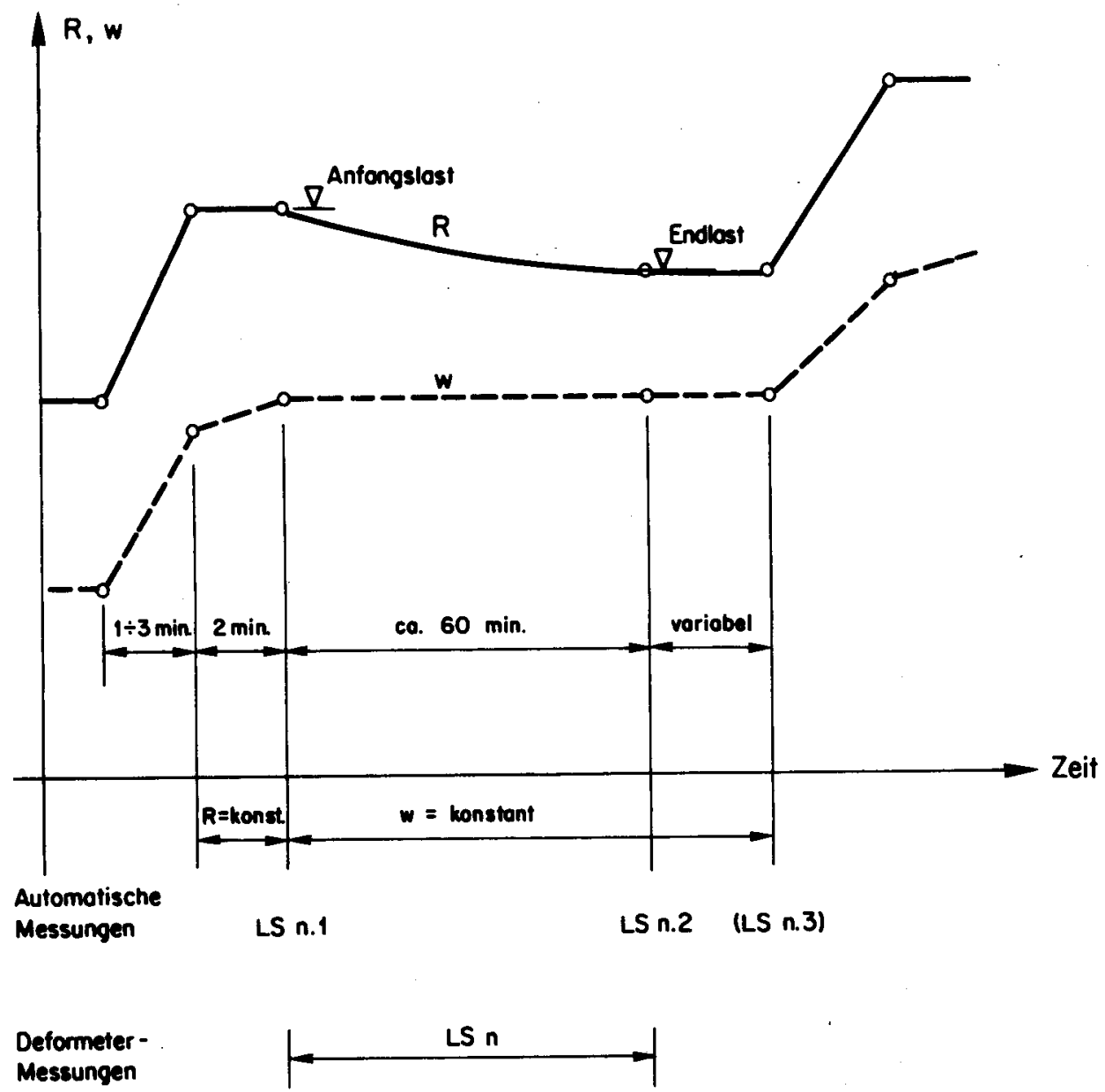

Messungen

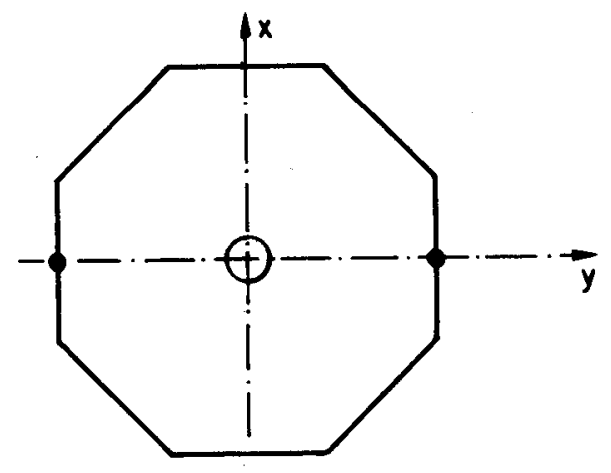

- Messung der Durchbiegung w zur Versuchssteuerung $w=\frac{w_{1}+w_{2}}{2}$

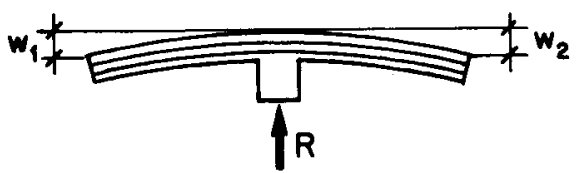




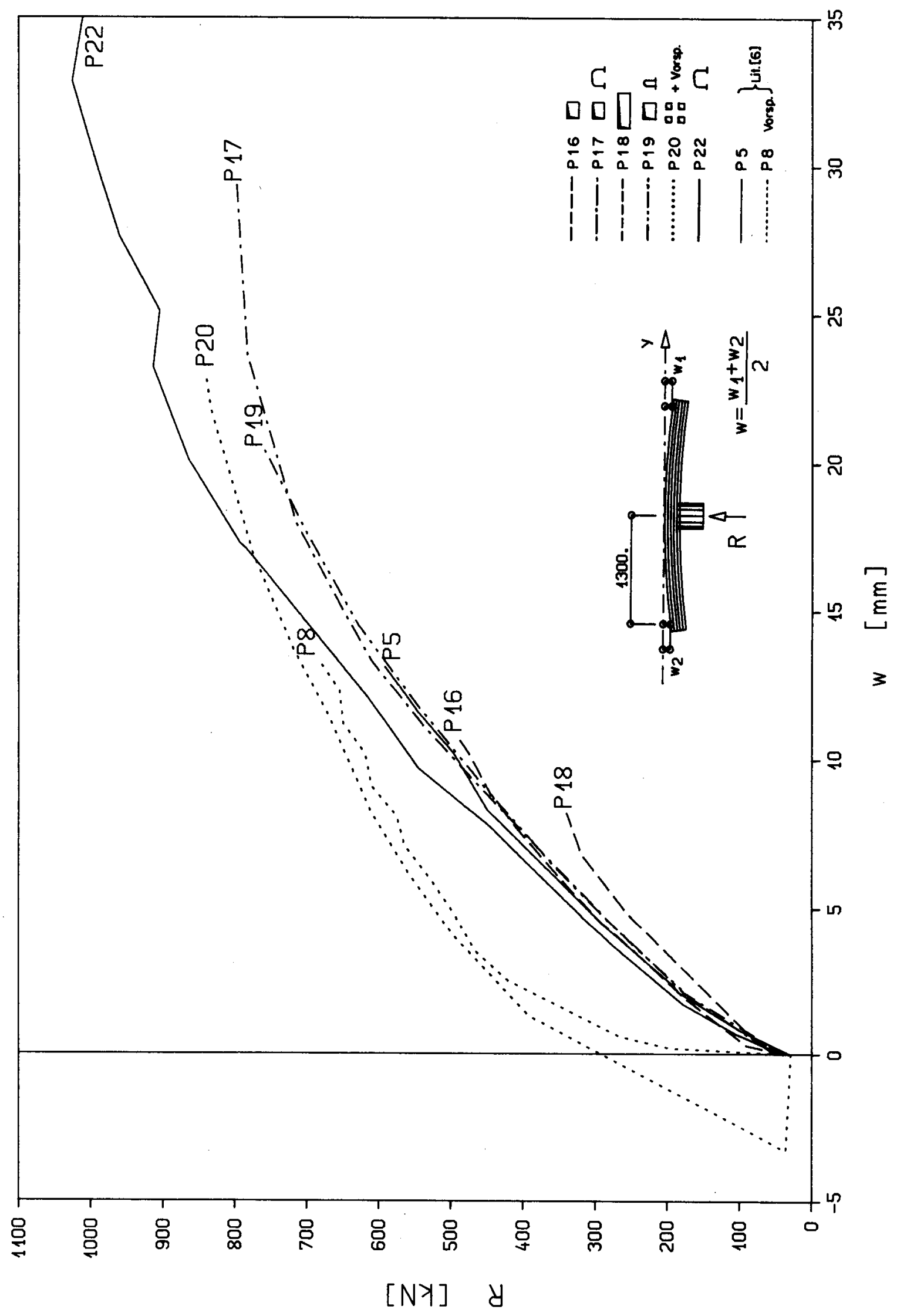

B1ld 22: Last-Durchbiegungsverlauf der Platten P 16 bis P 20, P 22 sowle verglelchswelse der Platten $P 5$ und $P 8$ 


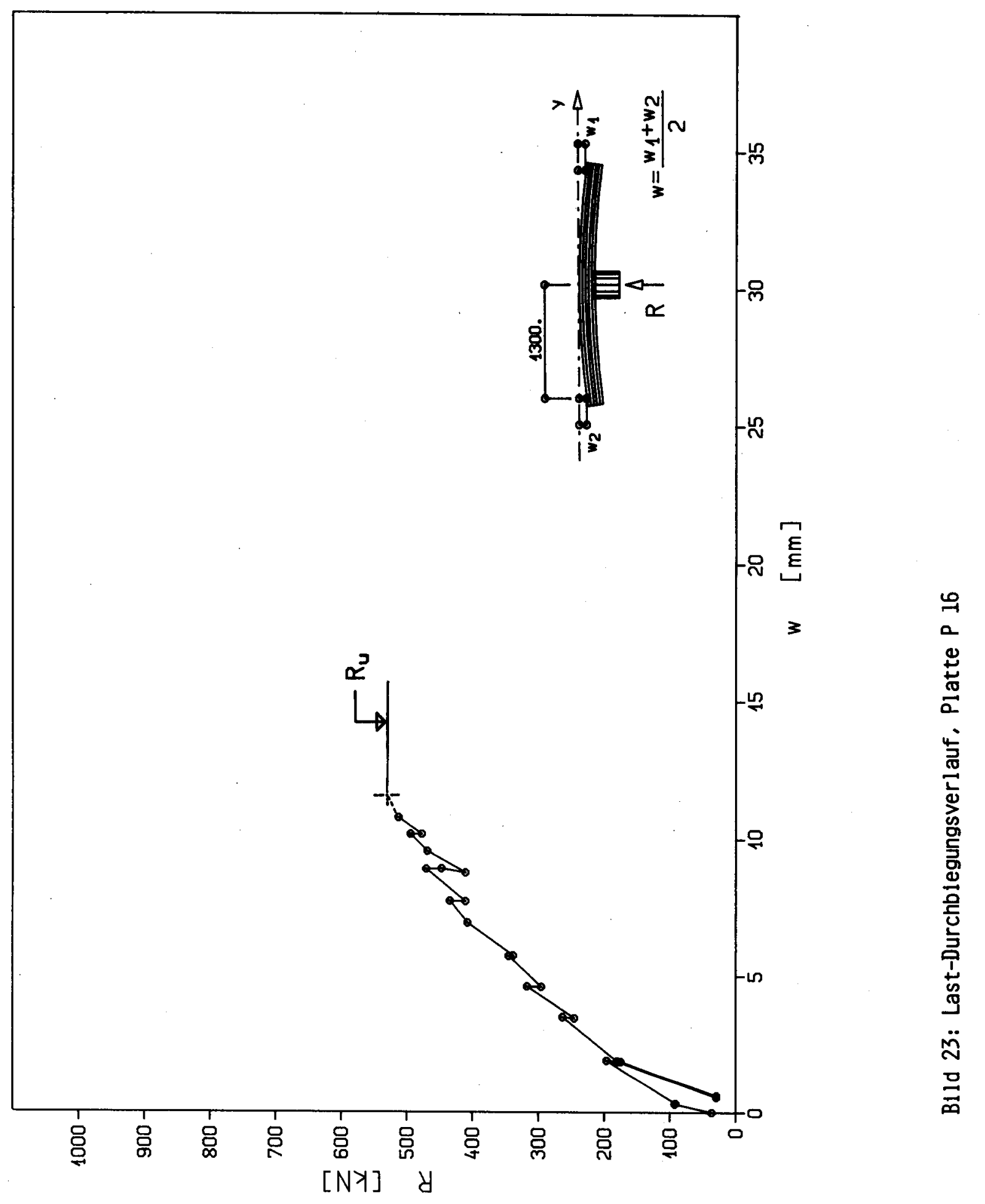

\begin{tabular}{|c|c|}
\hline 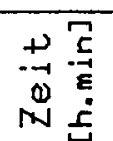 & 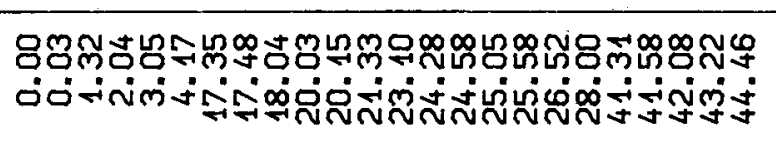 \\
\hline 3 管 & 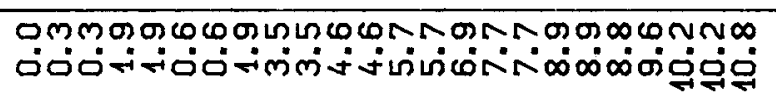 \\
\hline$\propto \frac{\bar{z}}{\underline{y}}$ & 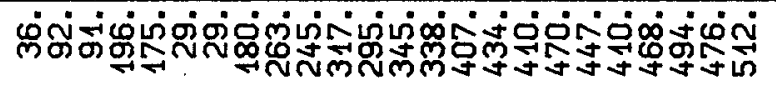 \\
\hline n & 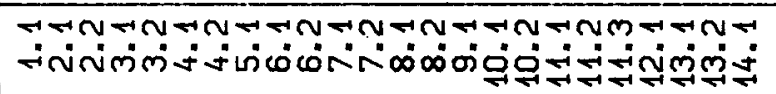 \\
\hline
\end{tabular}




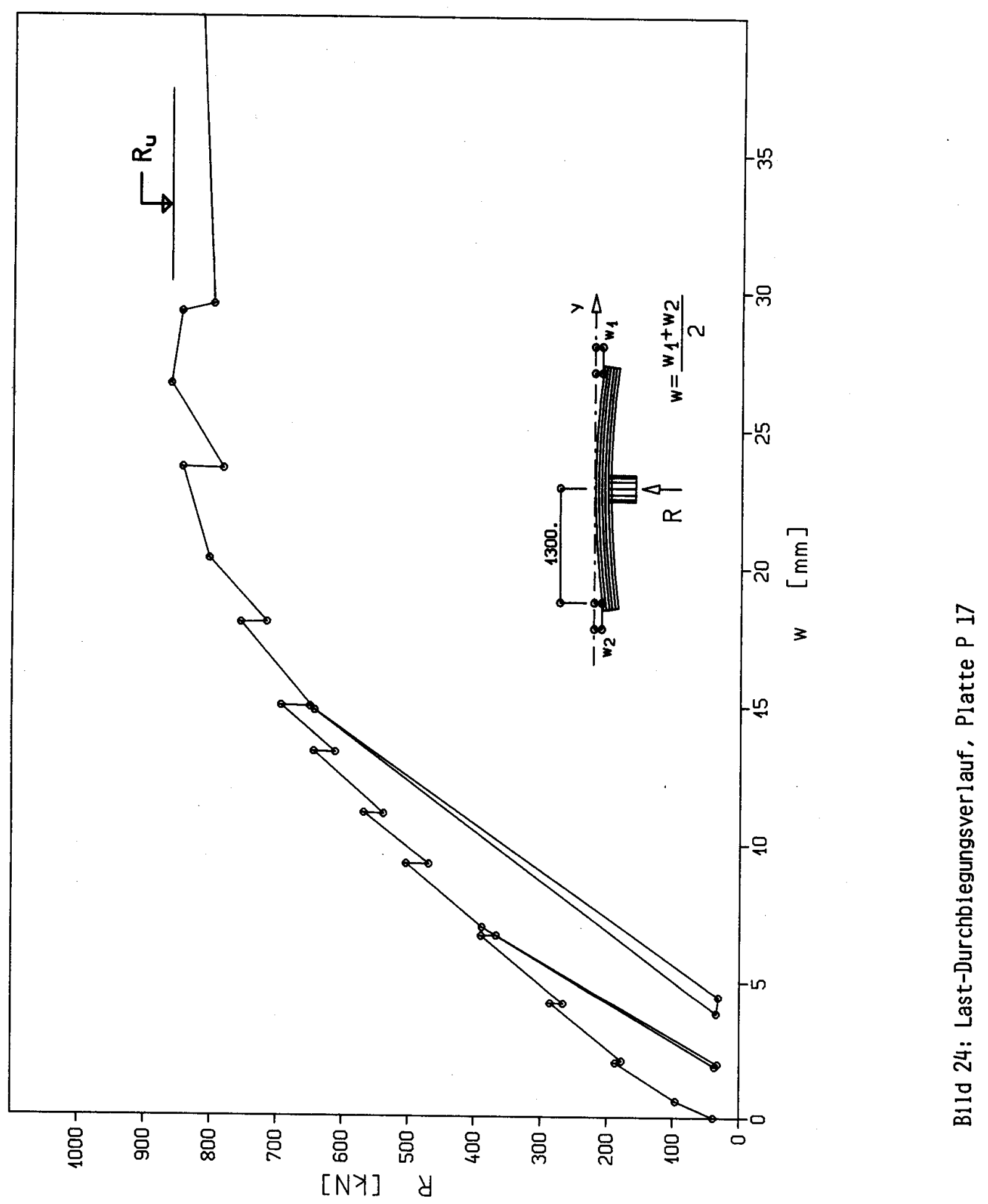

\begin{tabular}{|c|c|}
\hline 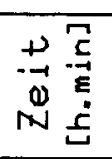 & 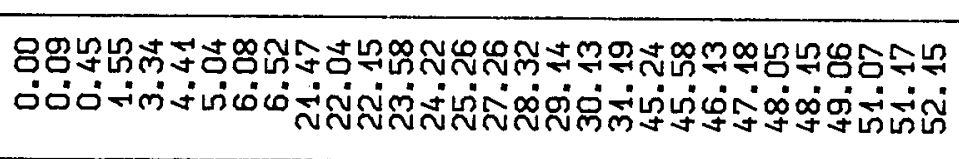 \\
\hline $3 \overline{\underline{E}}$ & 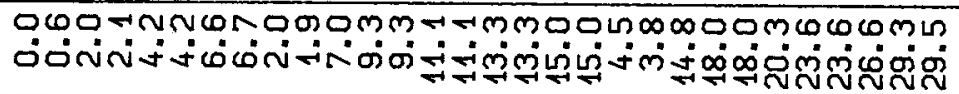 \\
\hline$\simeq \stackrel{\bar{z}}{\underline{4}}$ & लंம் \\
\hline s & 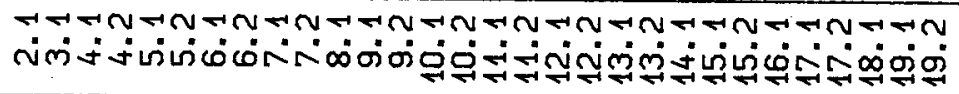 \\
\hline
\end{tabular}




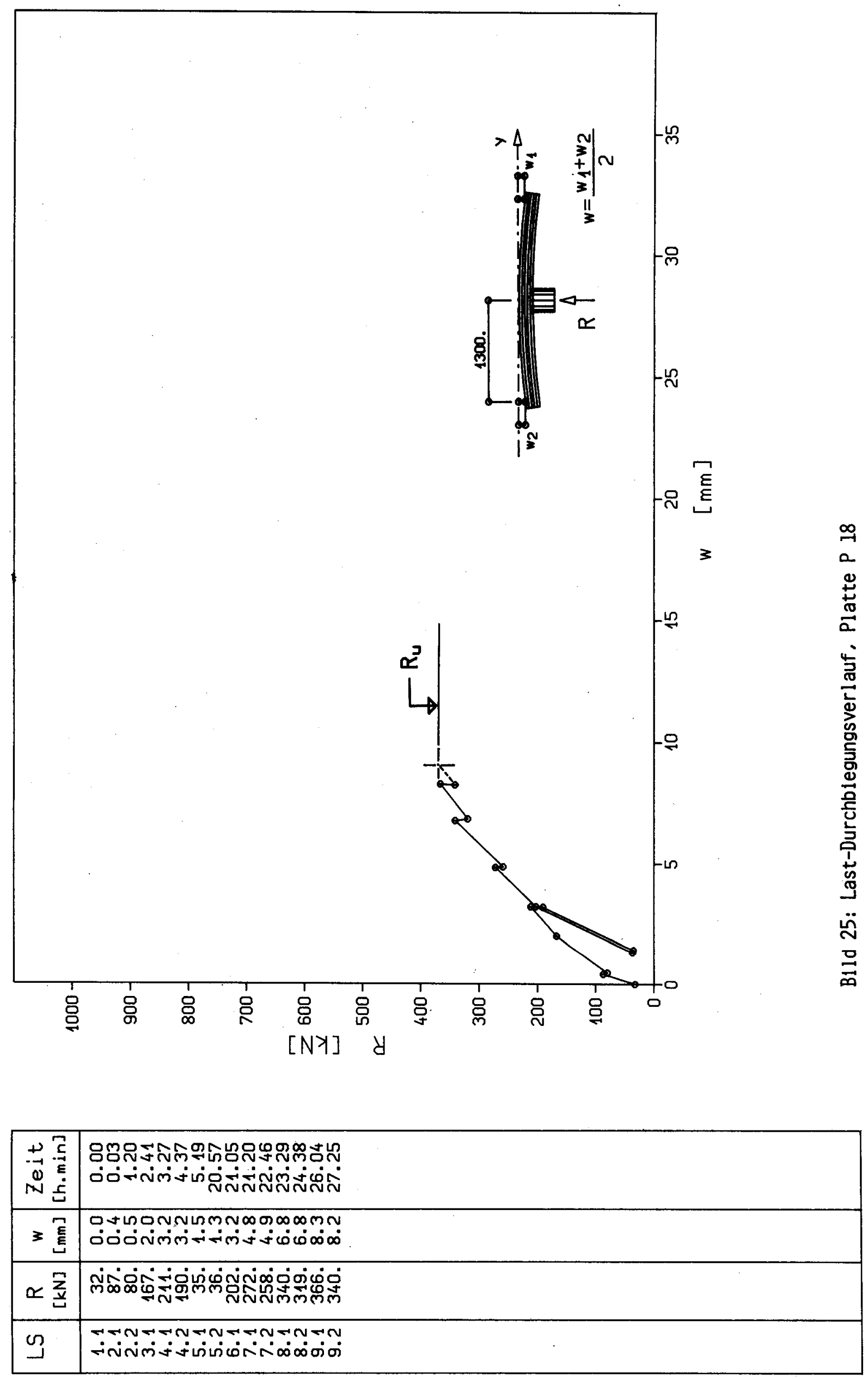




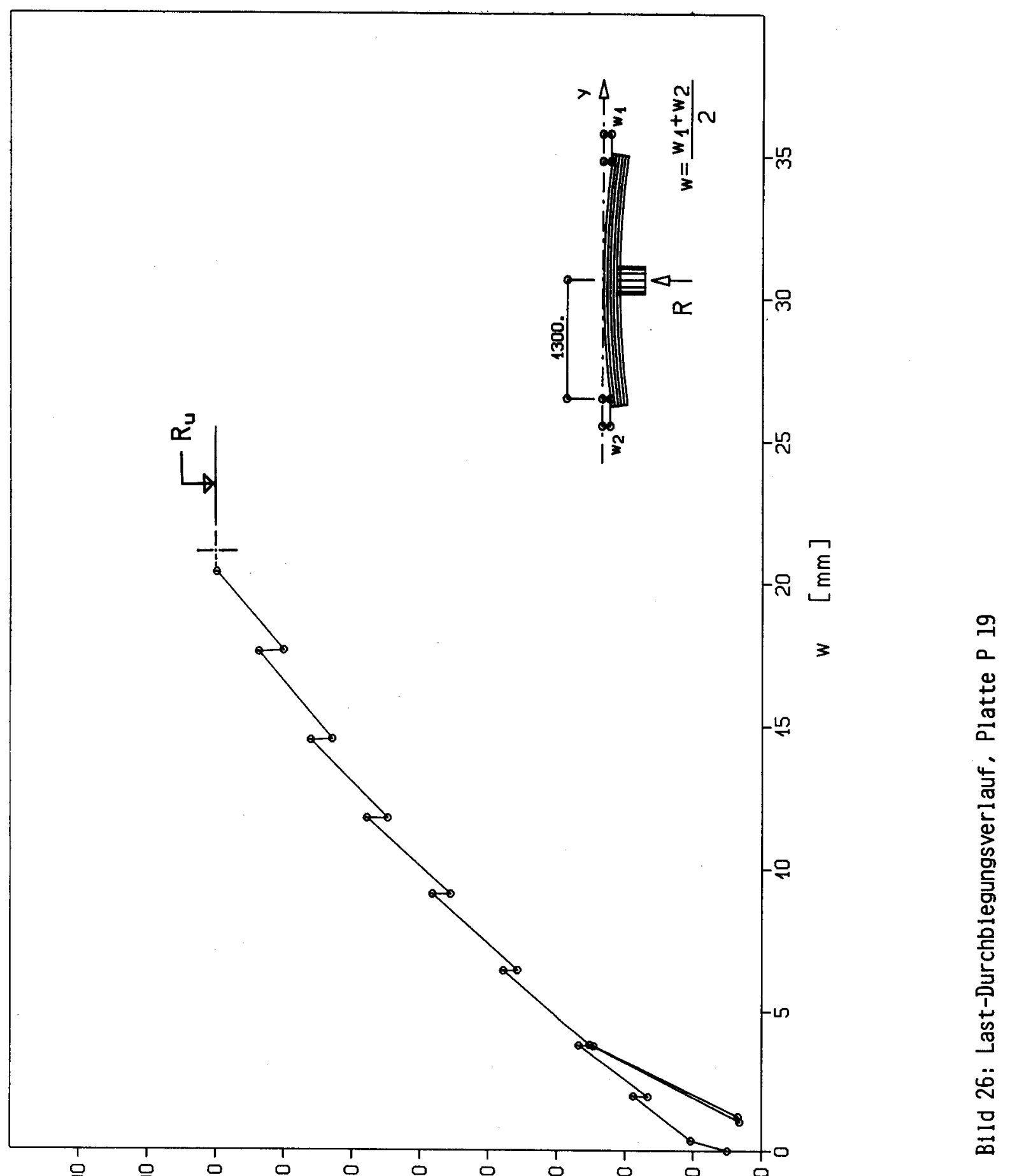

\begin{tabular}{|c|c|}
\hline 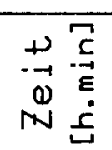 & 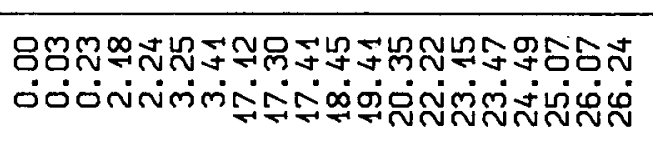 \\
\hline 3 㷃 & 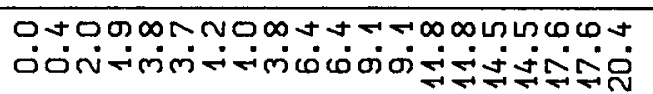 \\
\hline 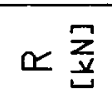 & 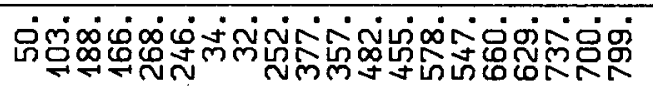 \\
\hline$\Omega$ & 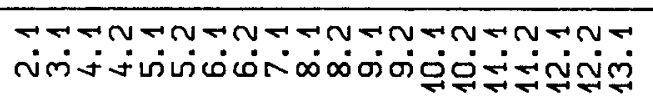 \\
\hline
\end{tabular}




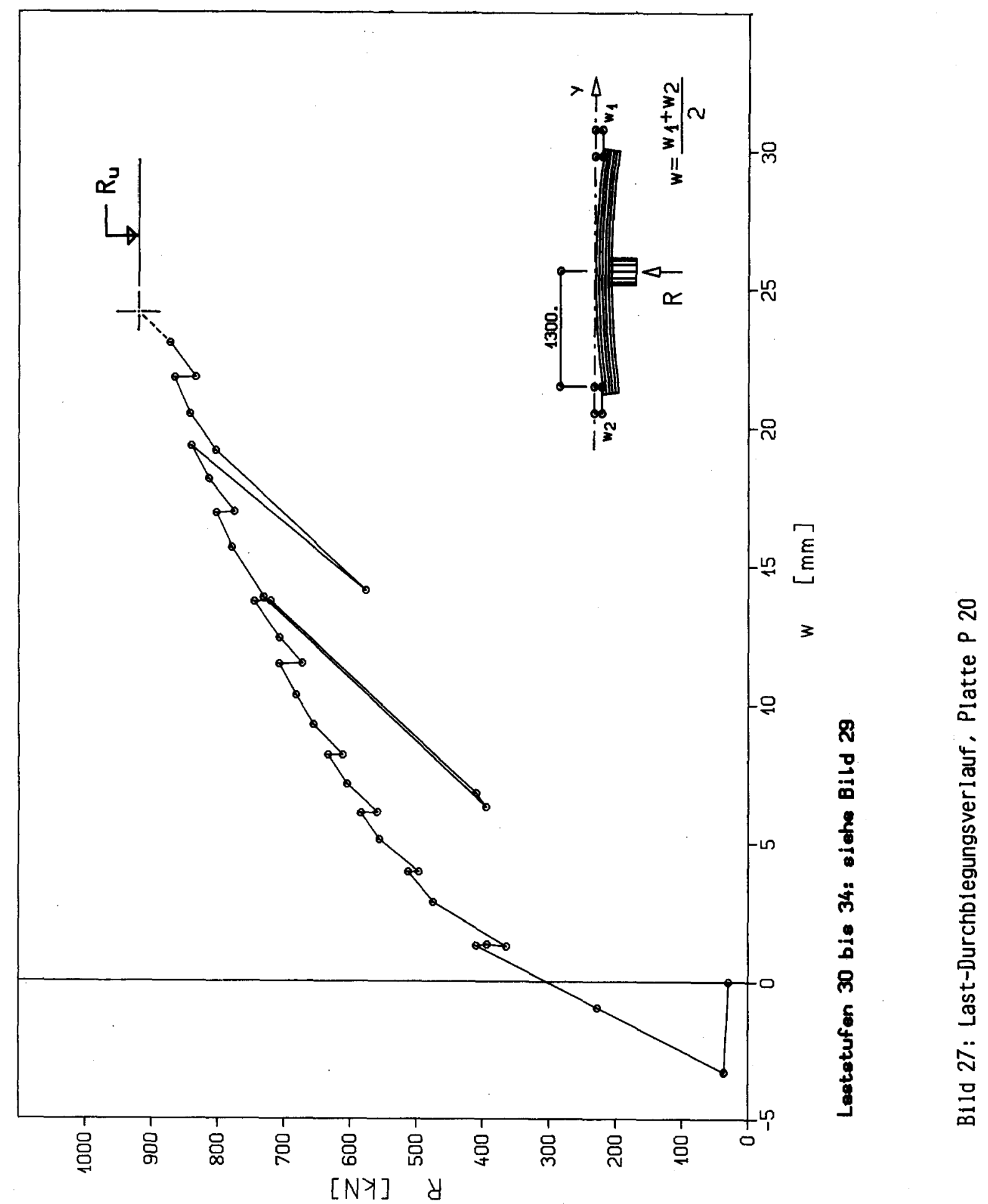

\begin{tabular}{|c|c|}
\hline 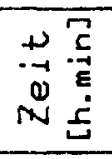 & 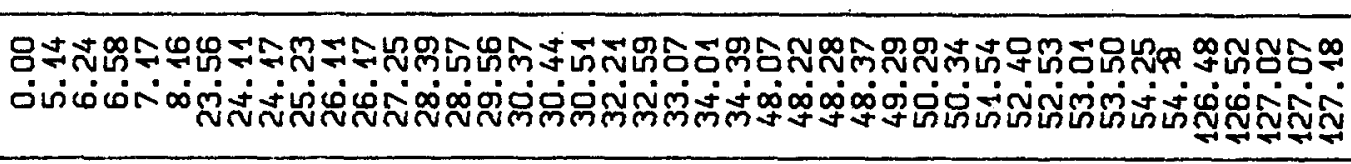 \\
\hline $3 \overline{\underline{\underline{E}}}$ & 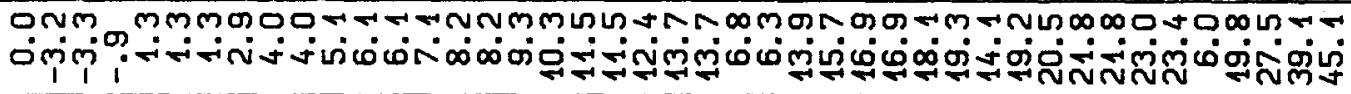 \\
\hline$\propto \stackrel{\frac{Z}{z}}{\underline{z}}$ & 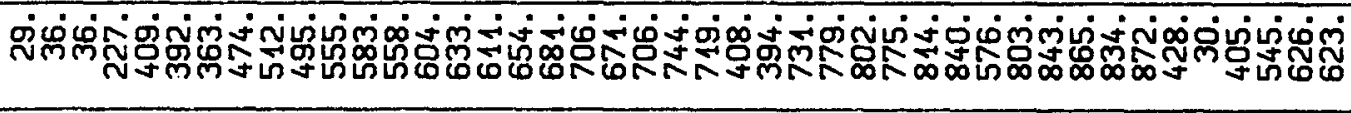 \\
\hline 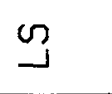 & 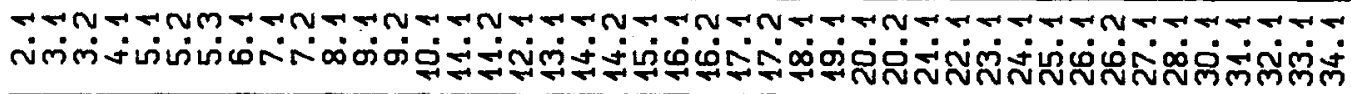 \\
\hline
\end{tabular}




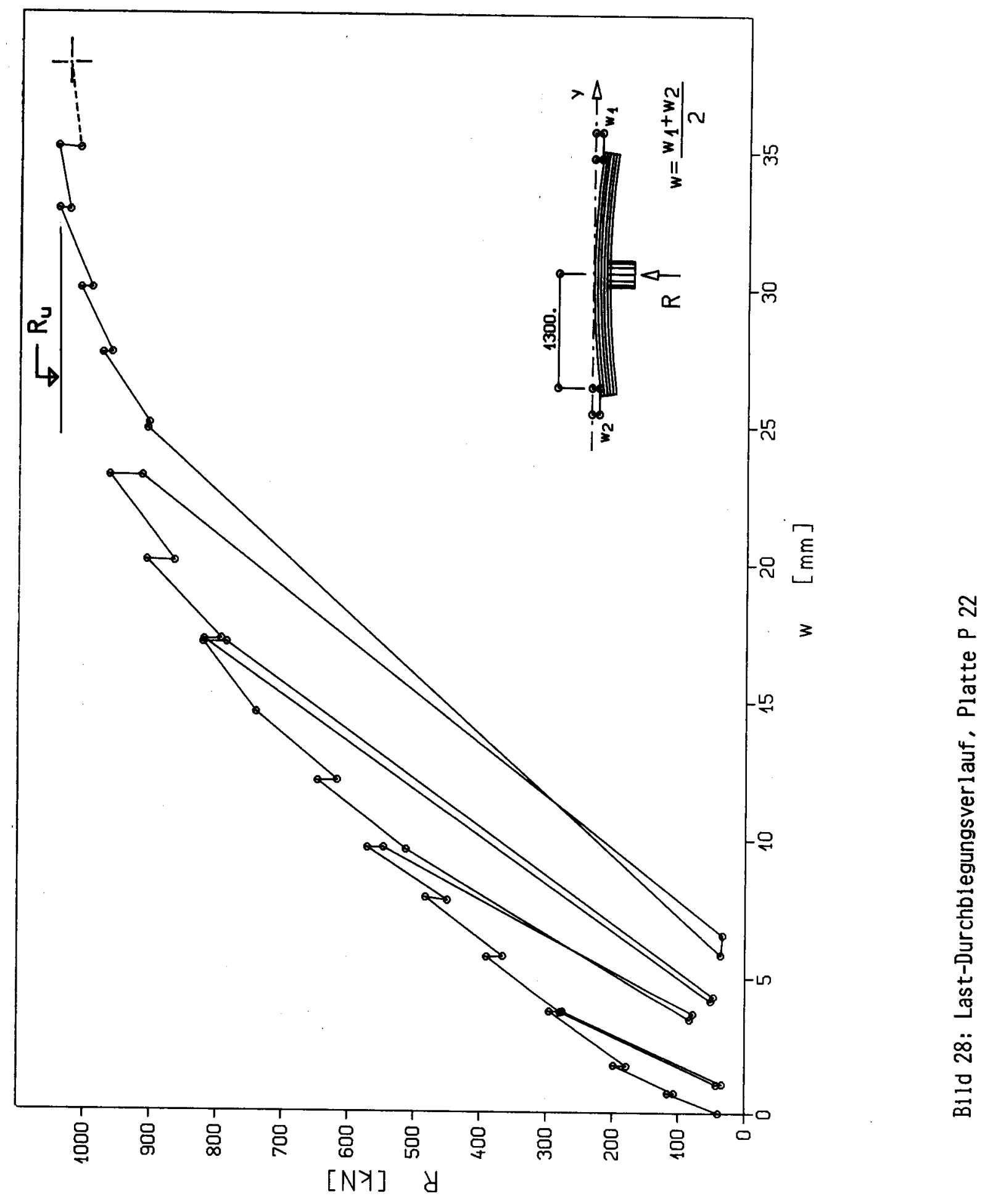

\begin{tabular}{|c|c|}
\hline 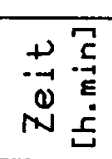 & 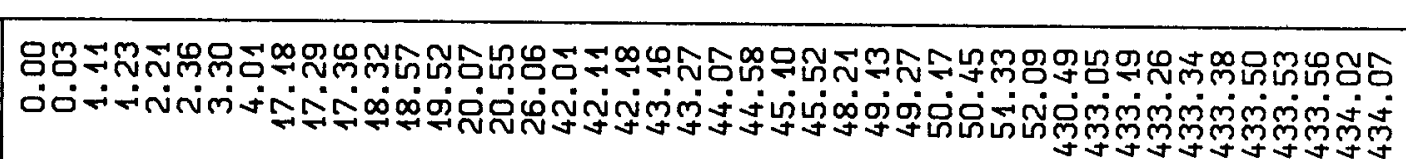 \\
\hline 3管 & 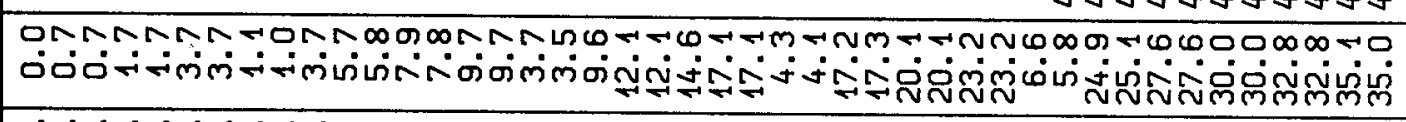 \\
\hline$\propto \frac{\bar{z}}{y}$ & 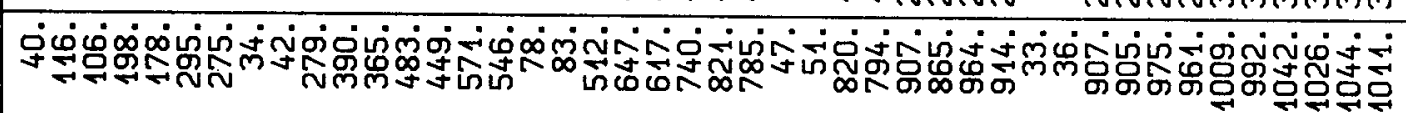 \\
\hline ב & 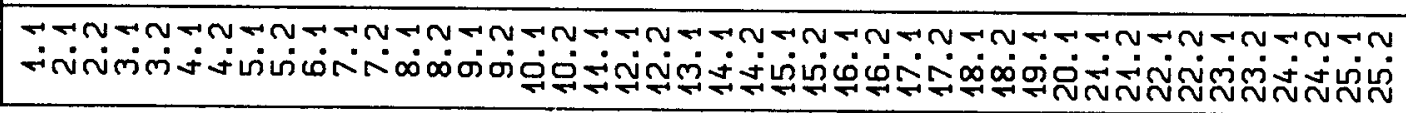 \\
\hline
\end{tabular}




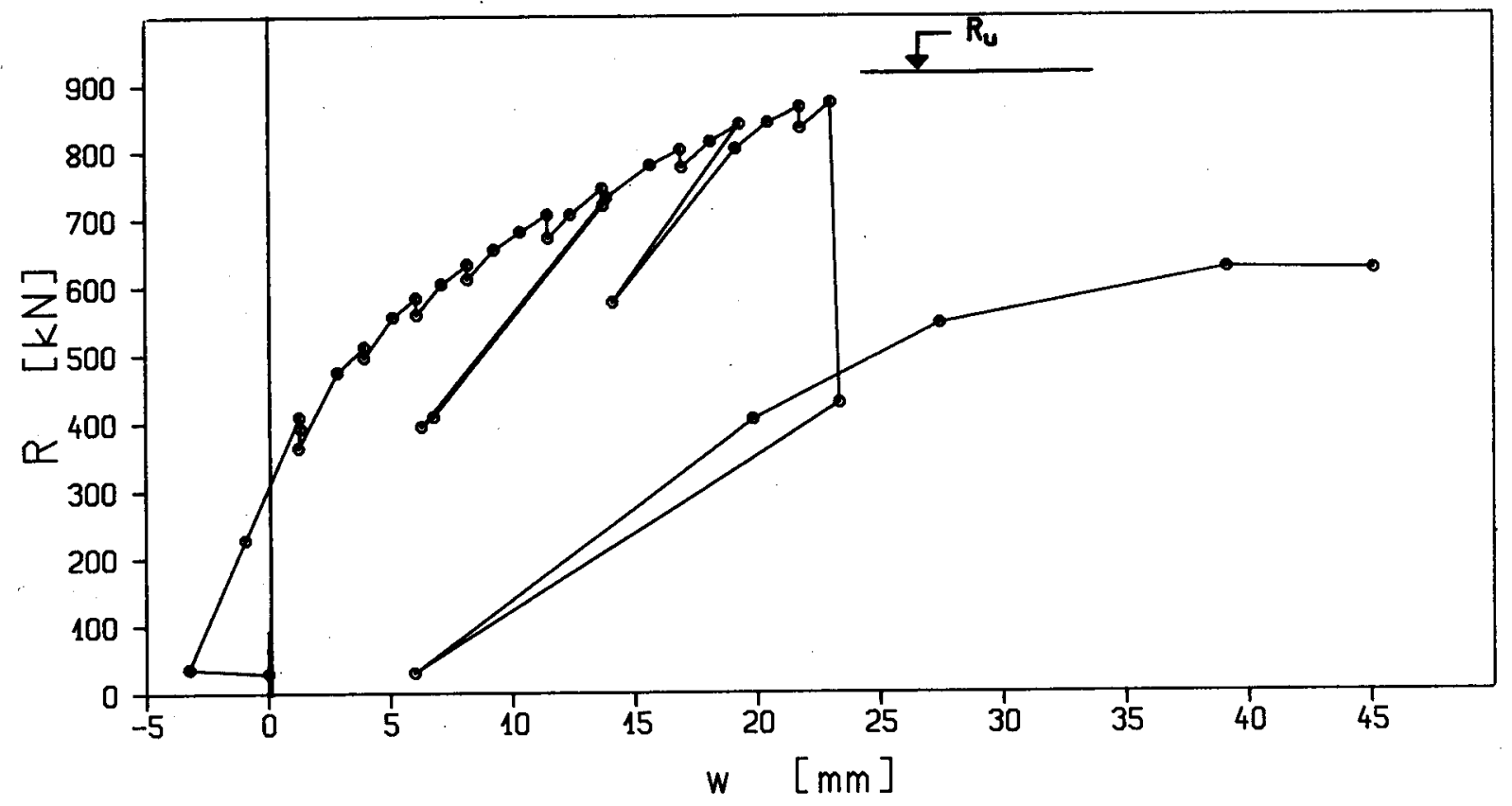

B1ld 29: Last-Durchblegungsverlauf, Platte P 20 (mit Wiederbelastung nach dem Durchstanzen)

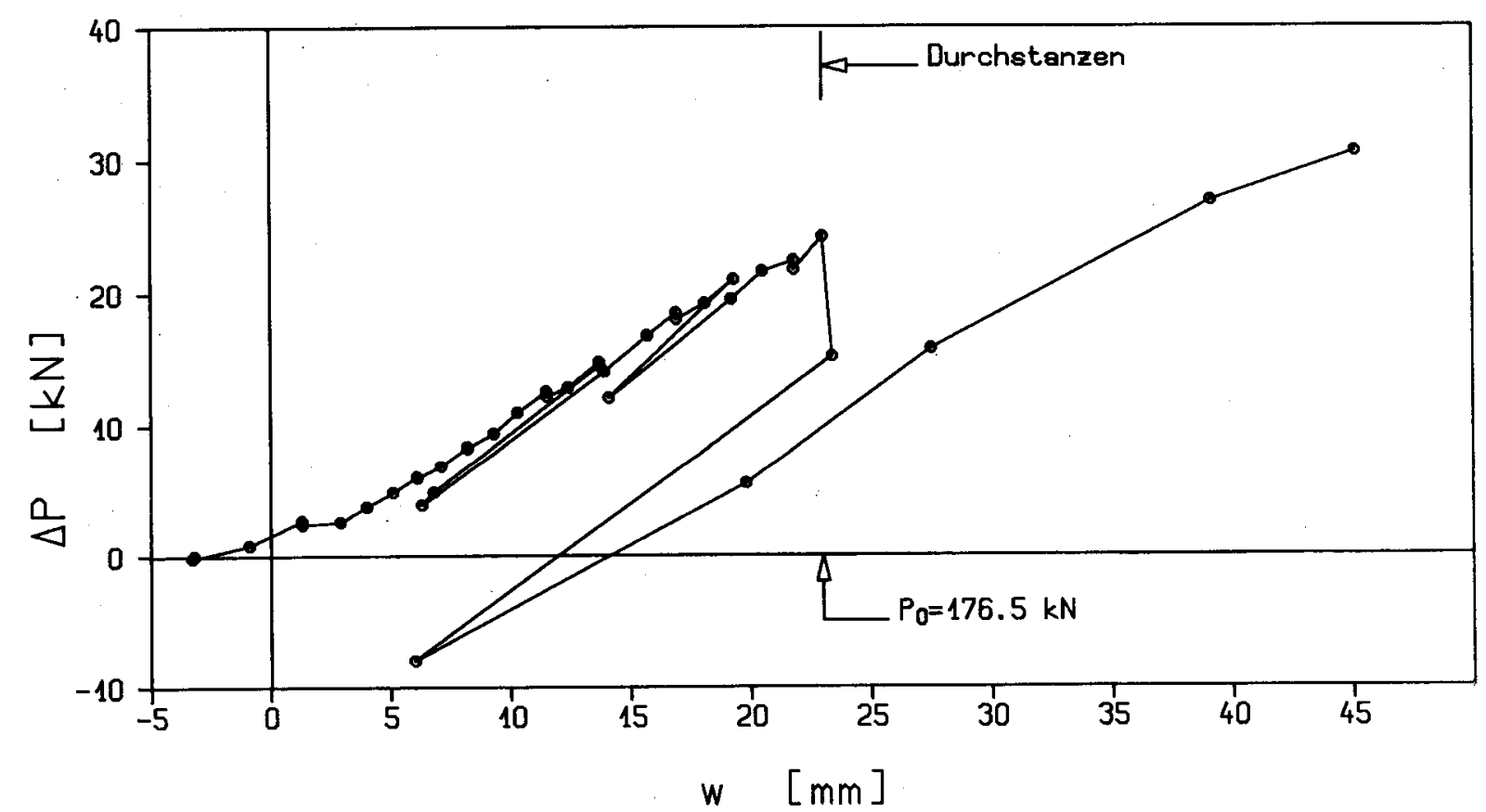

Bild 30: Mittlerer Zuwachs der Kabelkraft, Platte P 20 

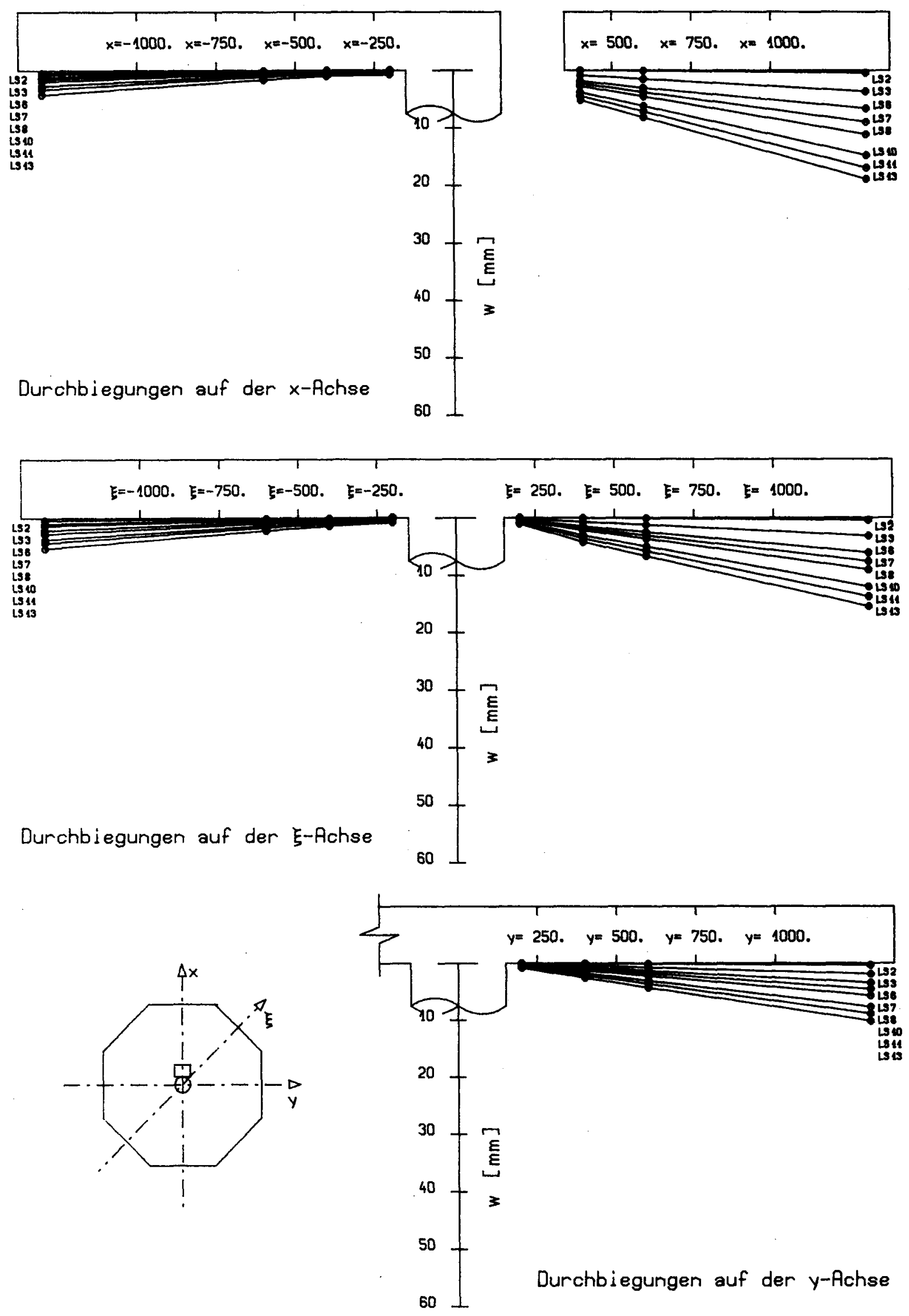

B1ld 31: Durchblegungen der Plattenunterseite, P 16 

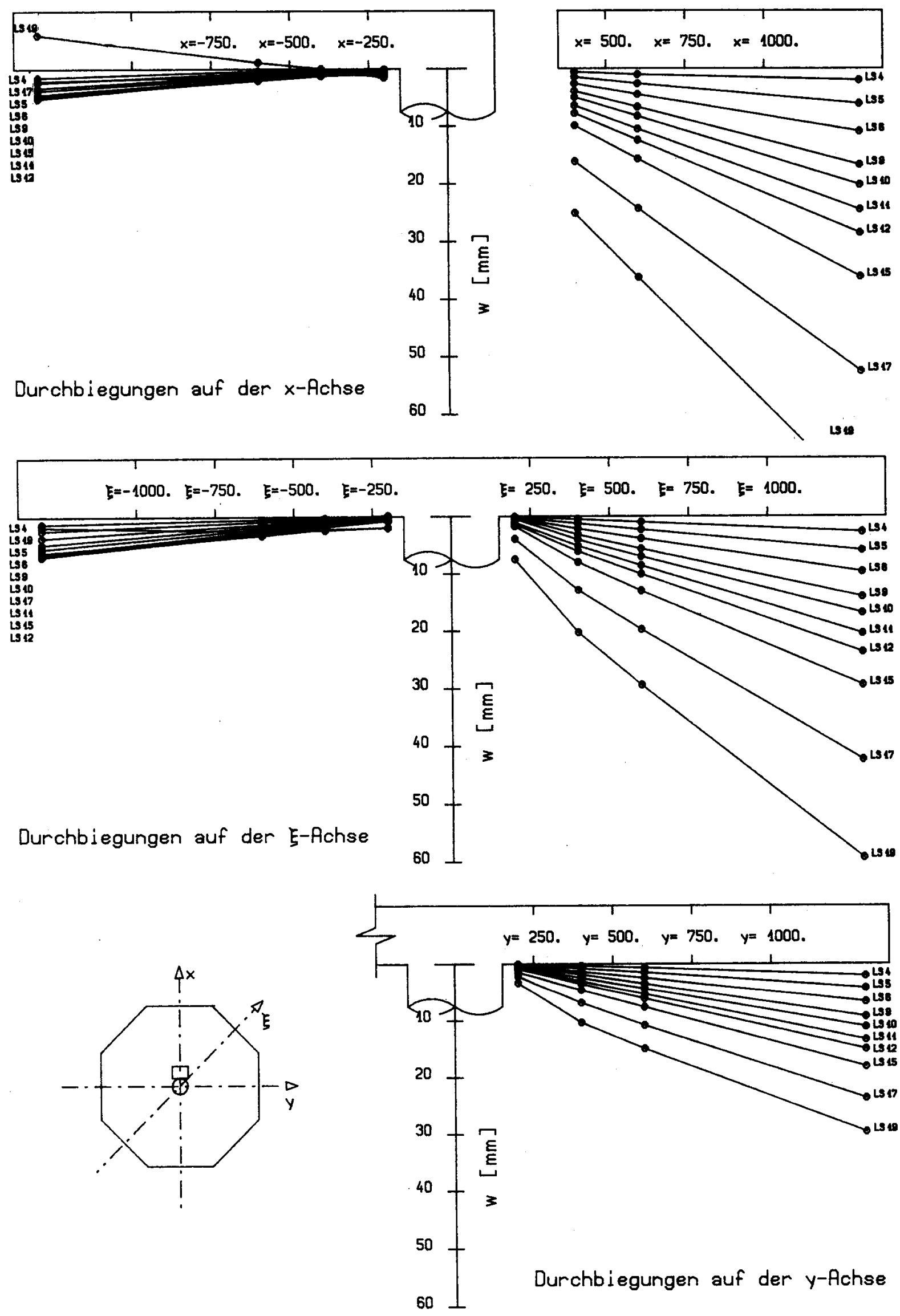

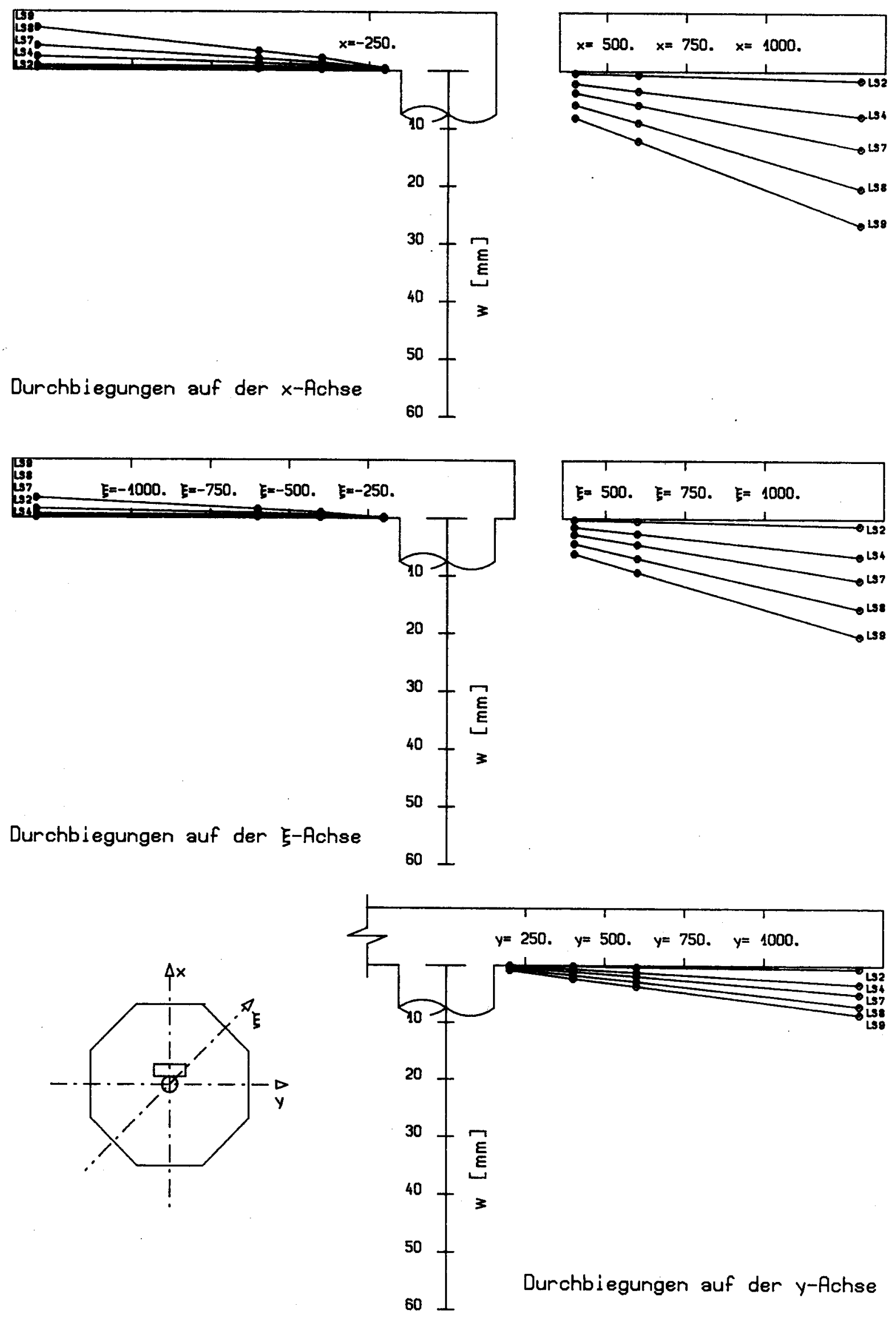

B1ld 33: Durchbiegungen der Plattenunterselte, P 18 

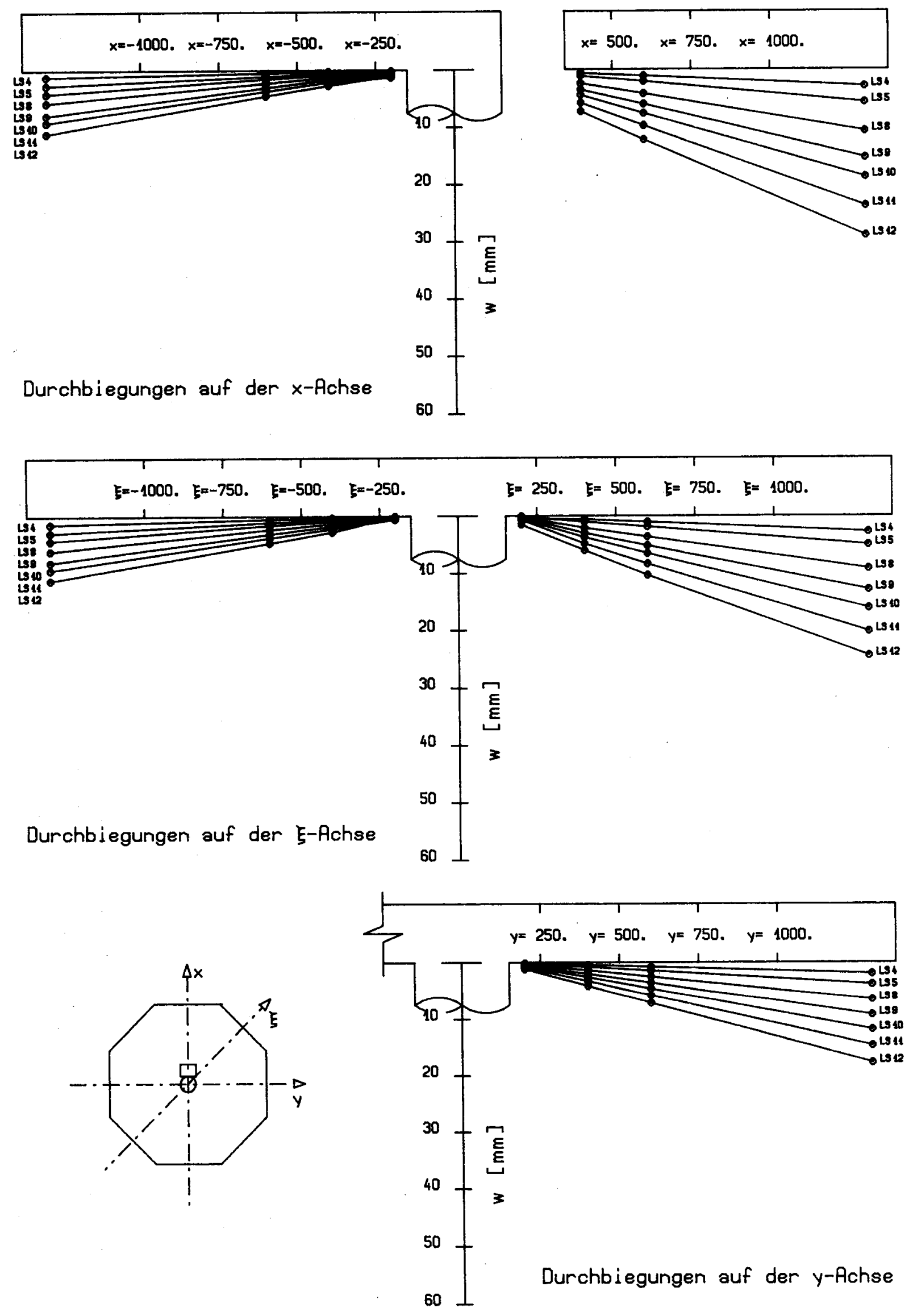

Bild 34: Durchbiegungen der Plattenunterseite, $P 19$ 

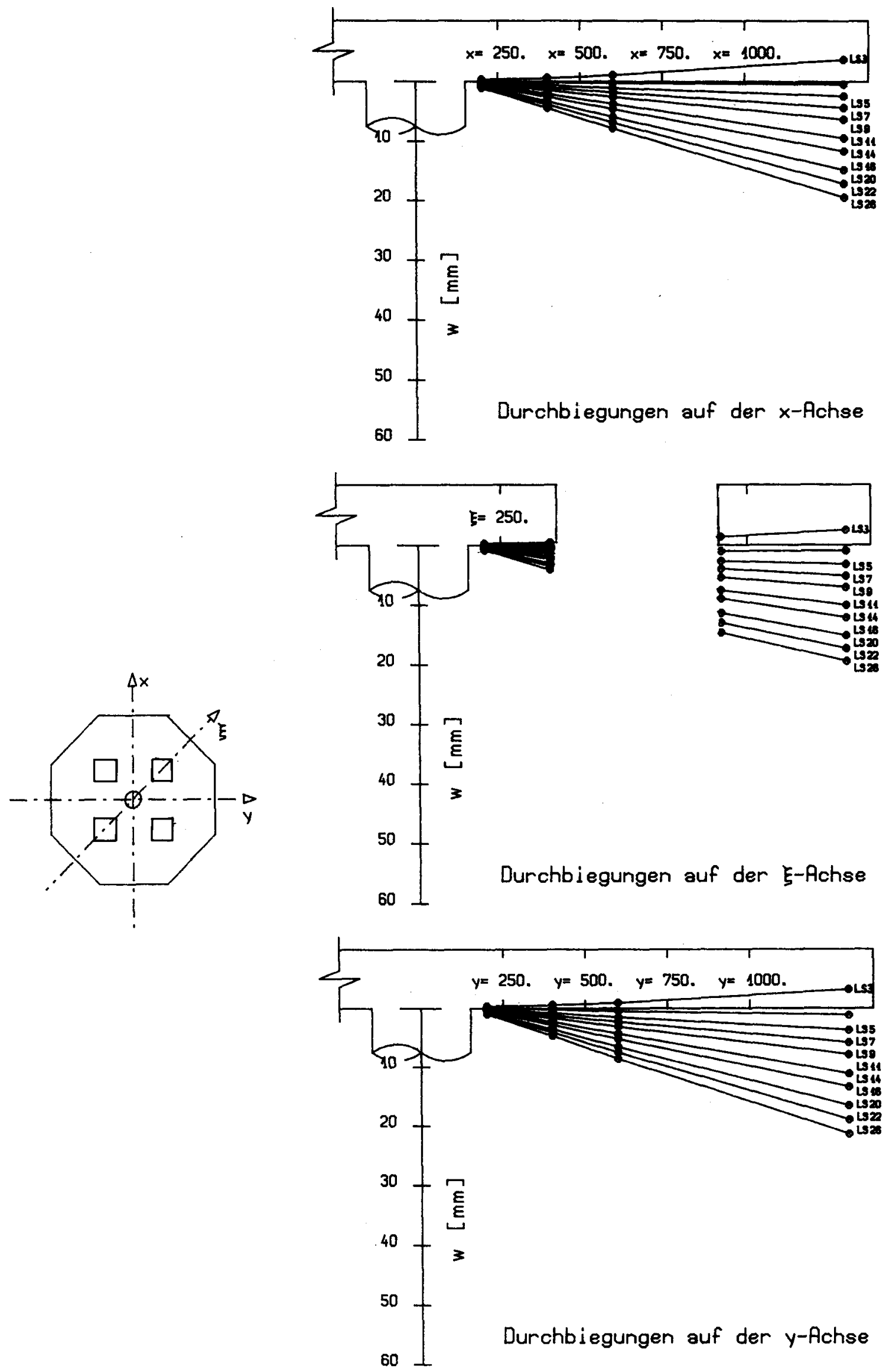

B11d 35: Durchblegungen der Plattenunterseite, $P 20$ 

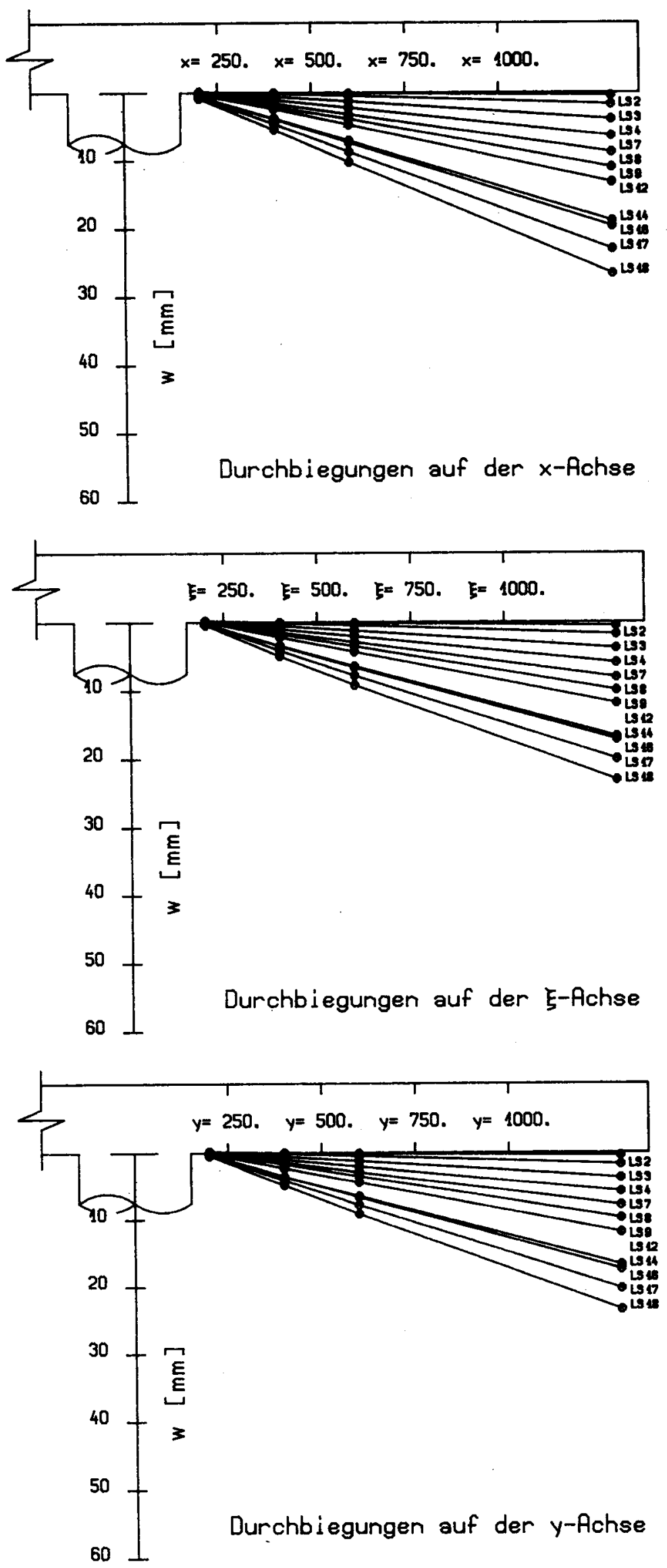

Bild 36: Durchblegungen der Plattenunterseite, $P 22$ 

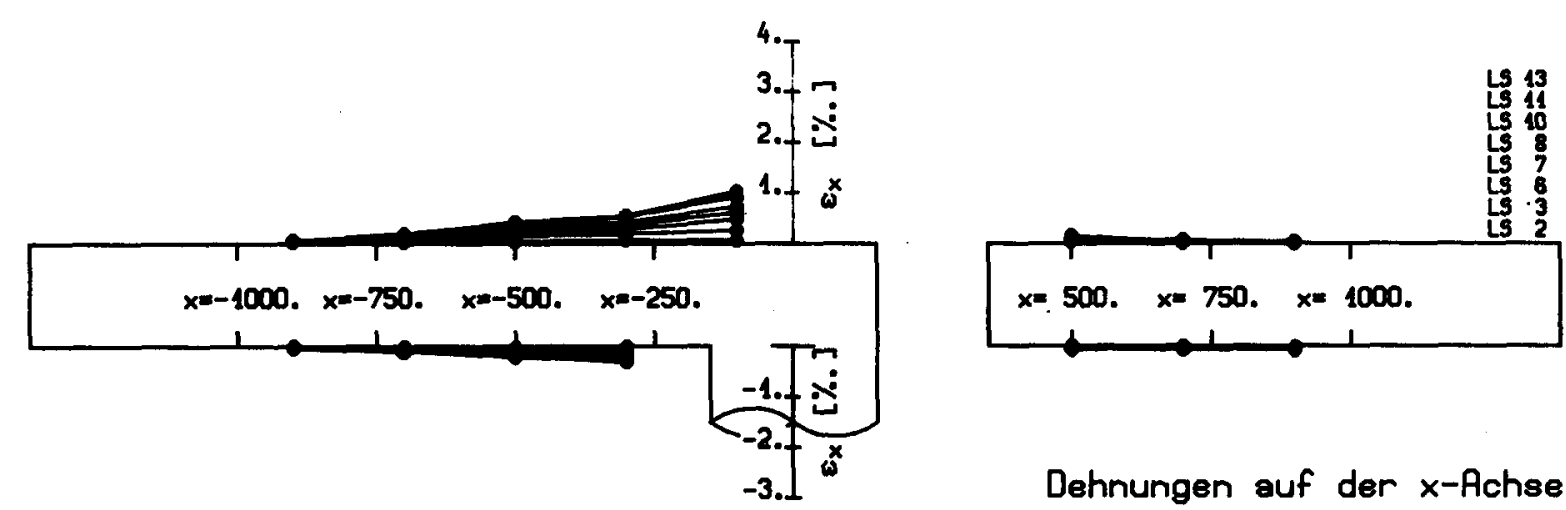

Dehnungen auf der $x$-Achse
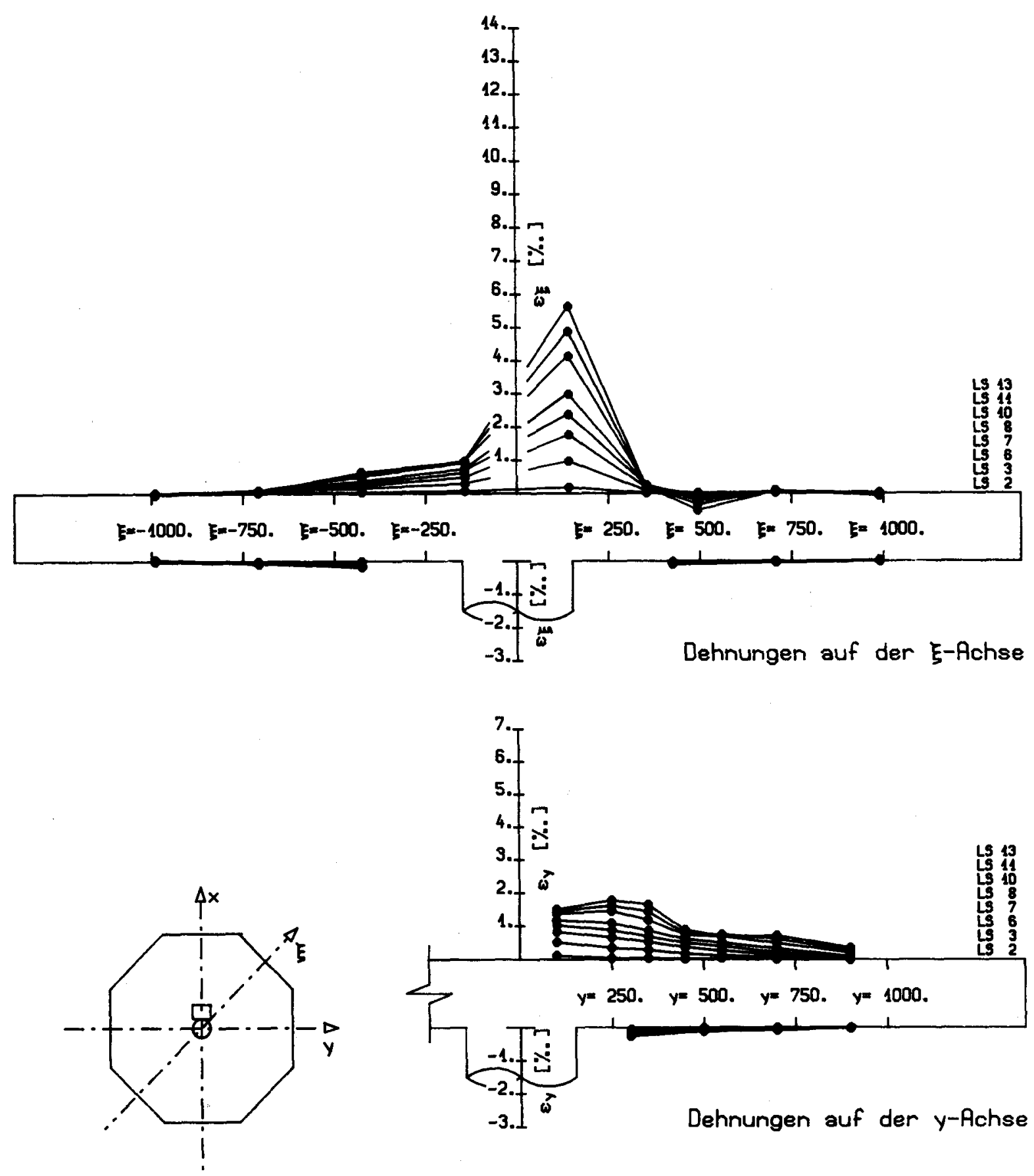

Bild 37: Mittlere radiale Dehnungen, Platte P 16 


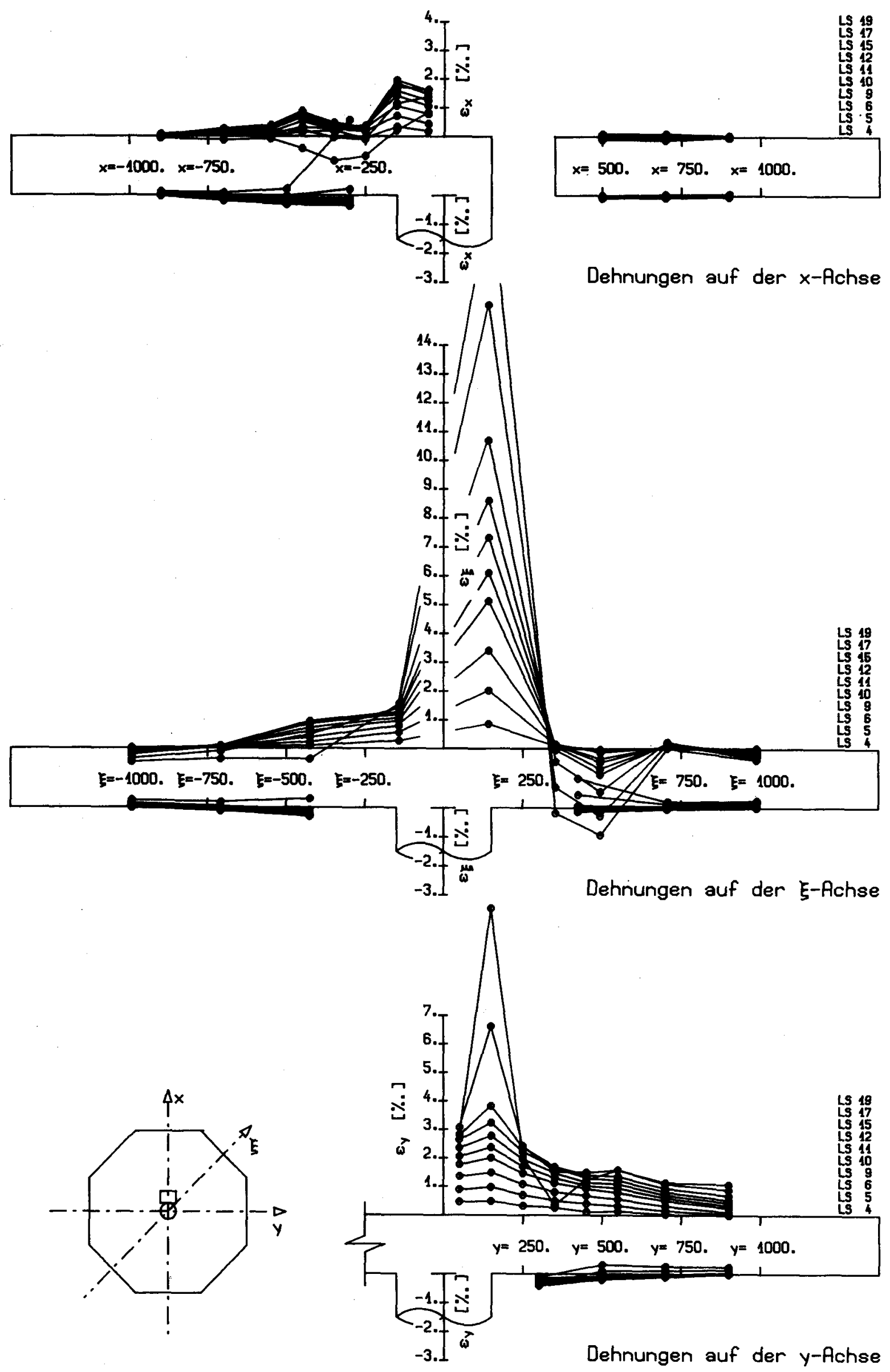

Blld 38: Mittlere radiale Dehnungen, Platte P 17 

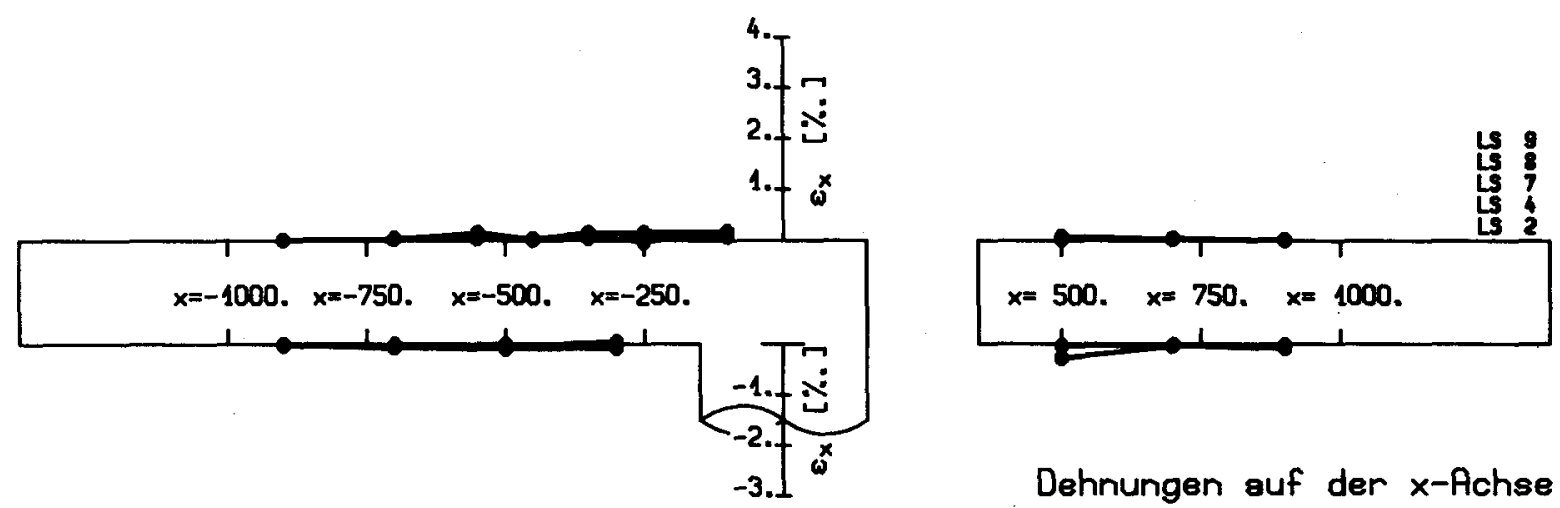

Dehnungen auf der $x$-Achse
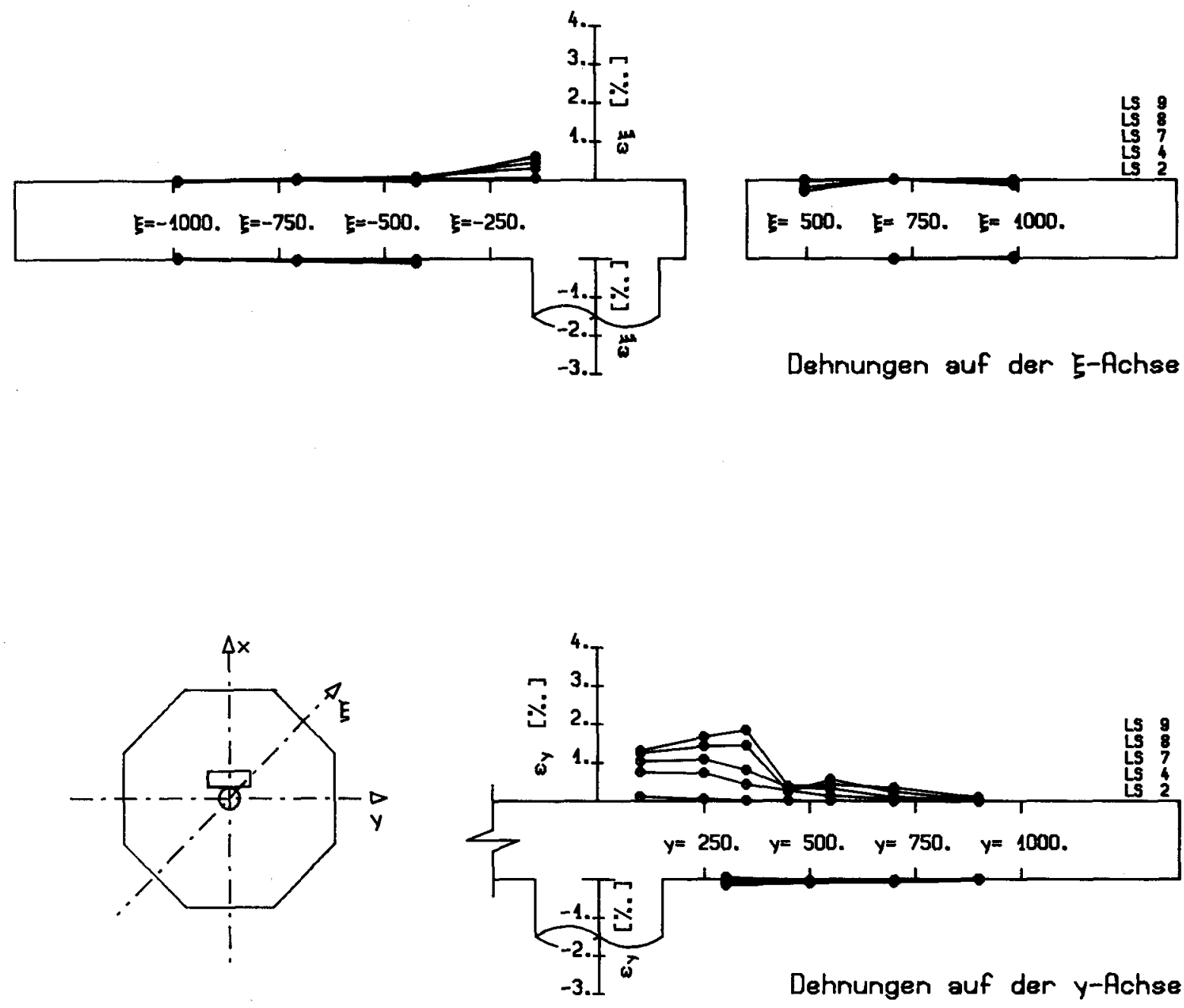

Bild 39: Mittlere radiale Dehnungen, Platte $P 18$ 

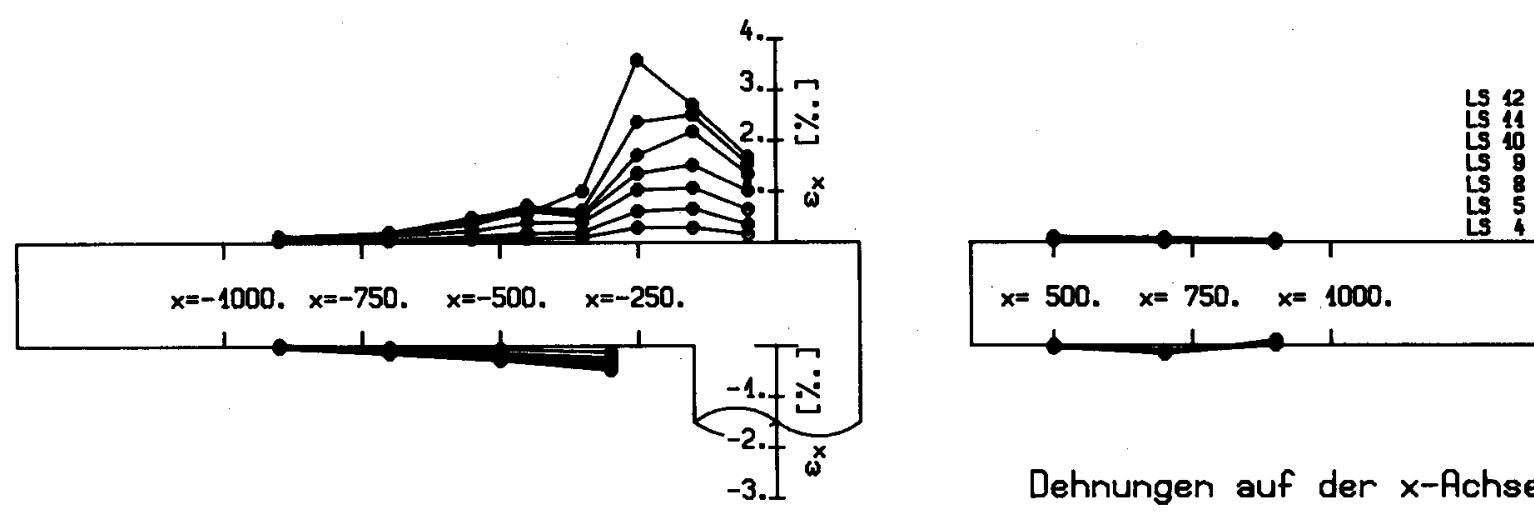

Dehnungen auf der $x$-Achse
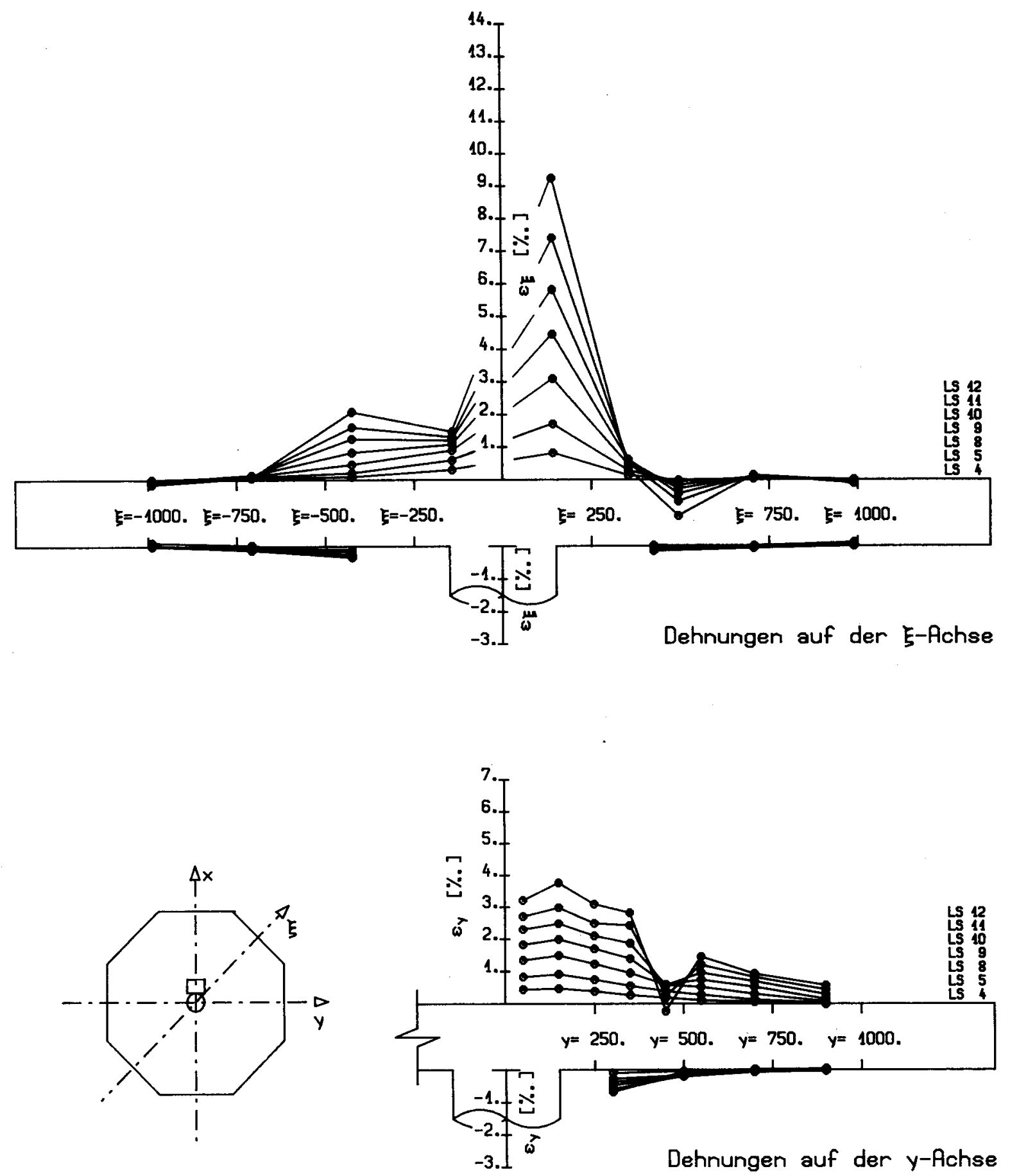

Bild 40: Mittlere radiale Dehnungen, Platte $P 19$ 

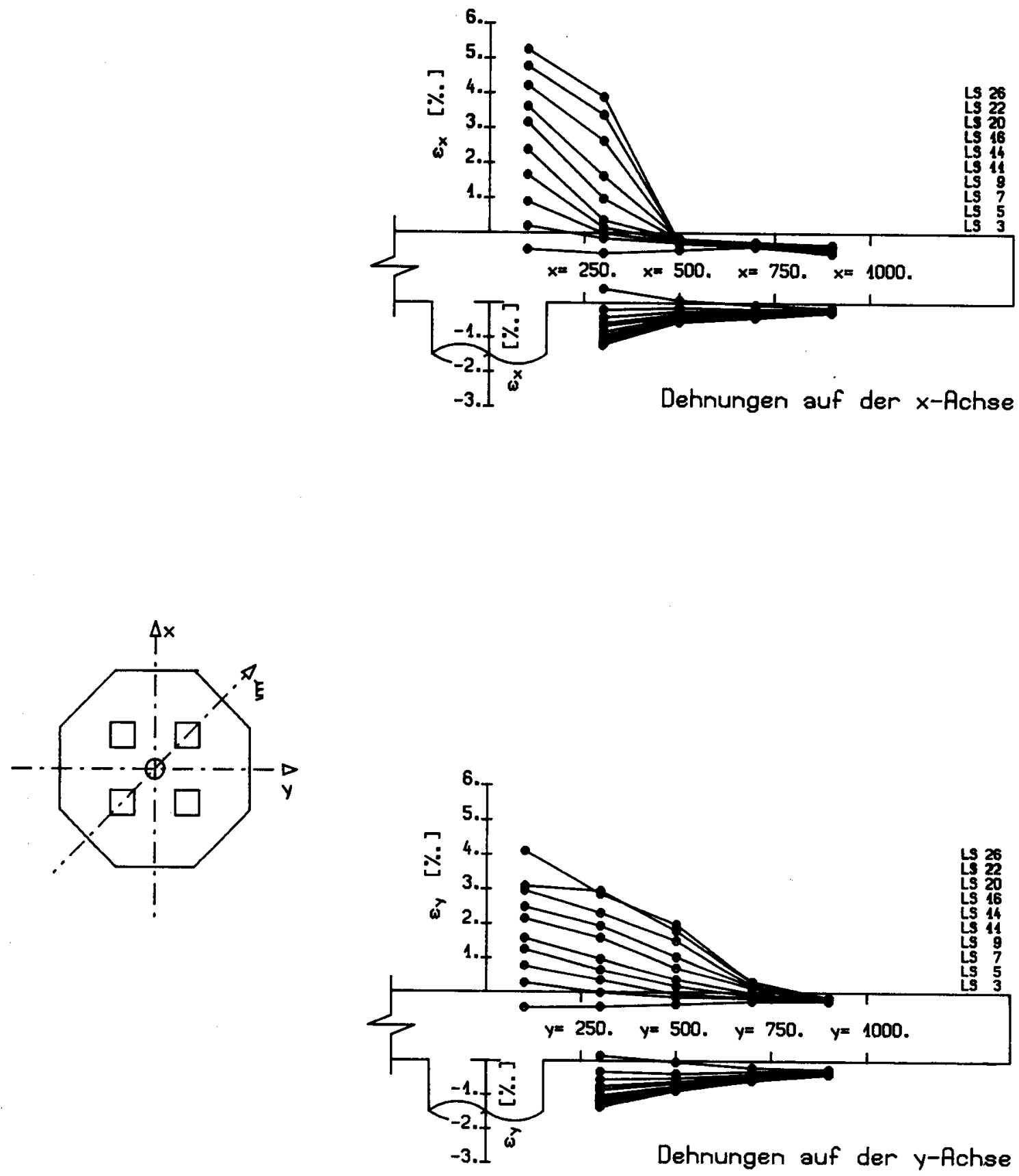

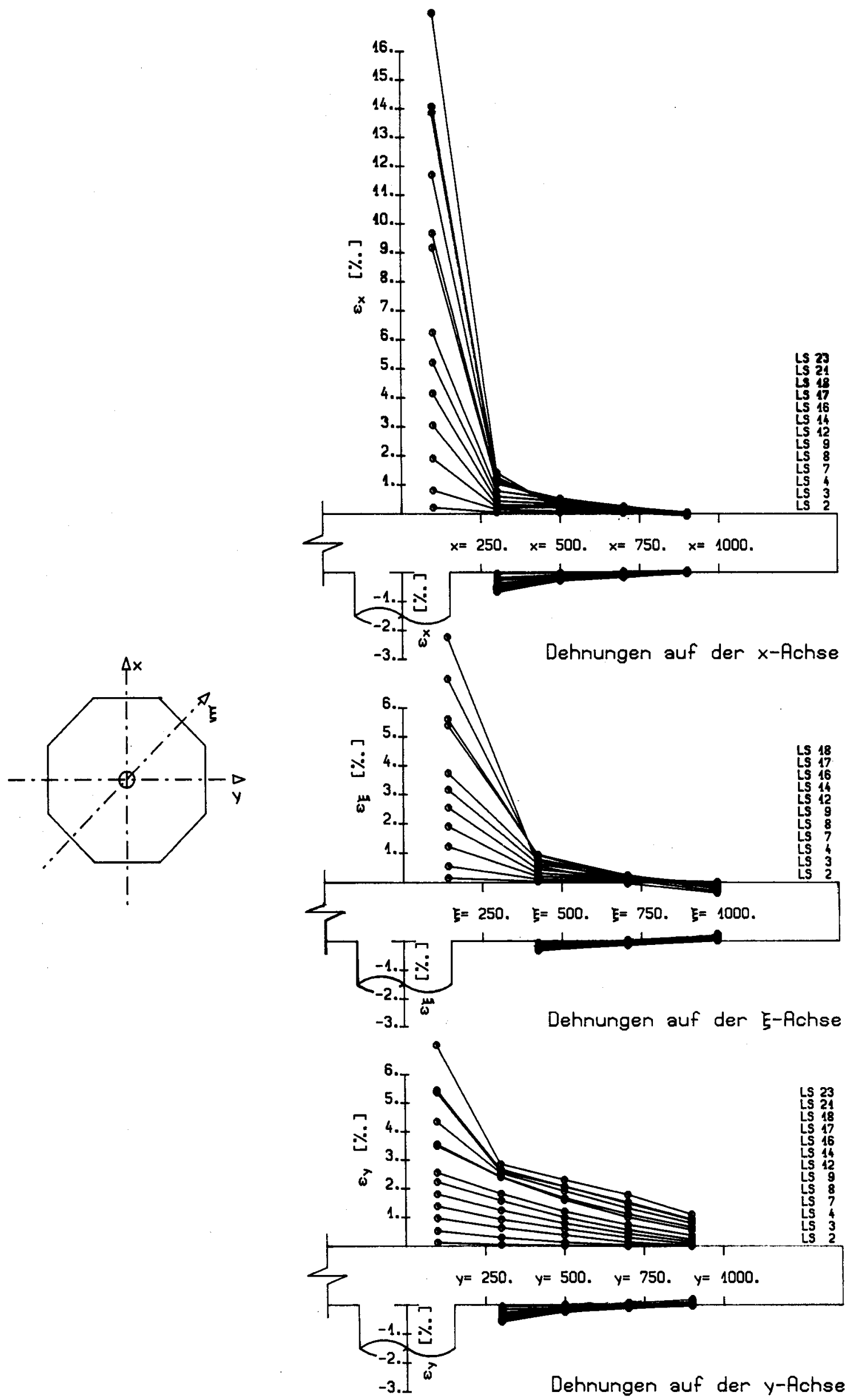

B11d 42: Mittlere radlale Dehnungen, Platte $P 22$ 

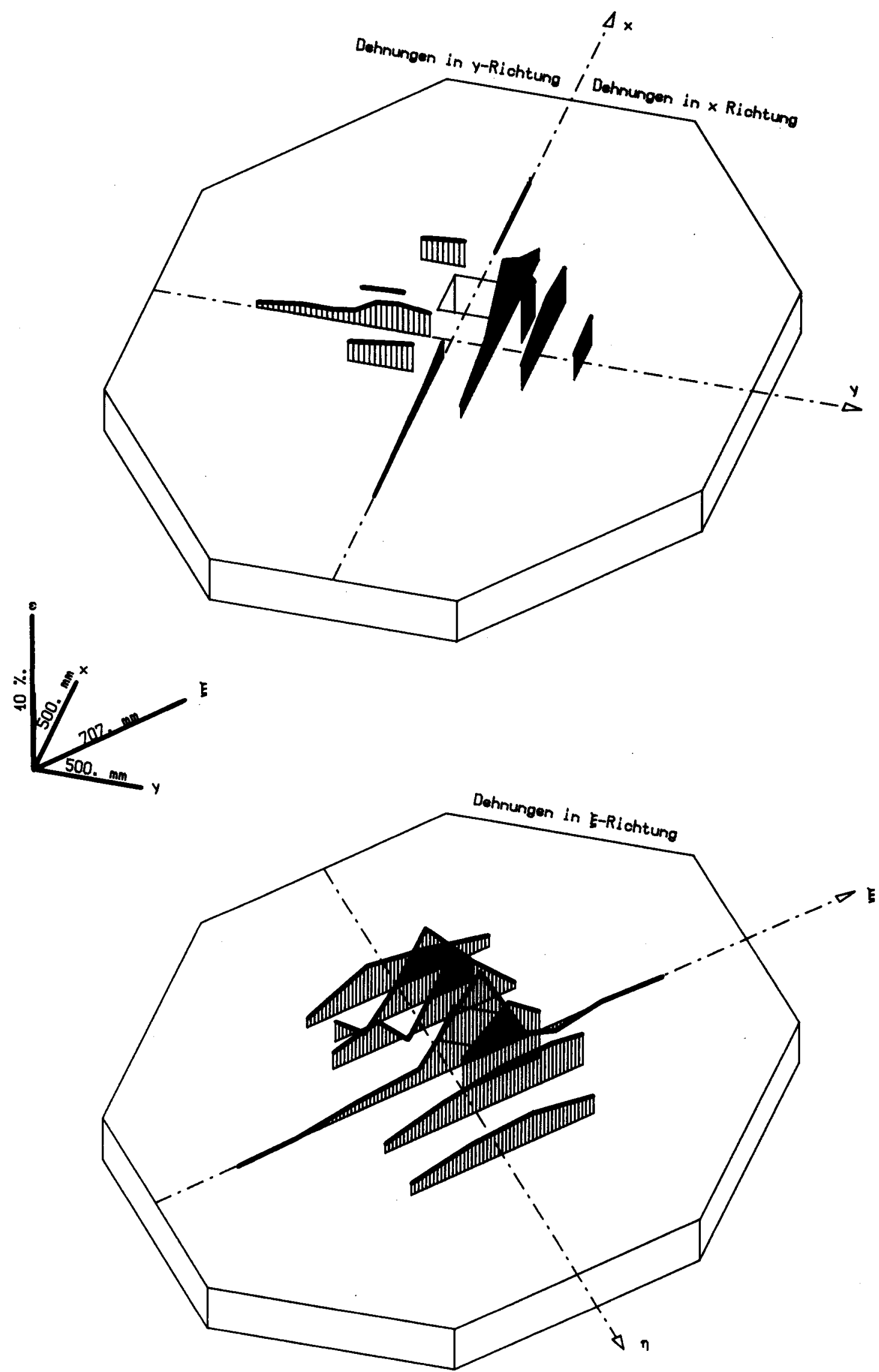

Bild 43: Mittlere Dehnungen der oberen Messebene, Platte P 16, Laststufe 13 

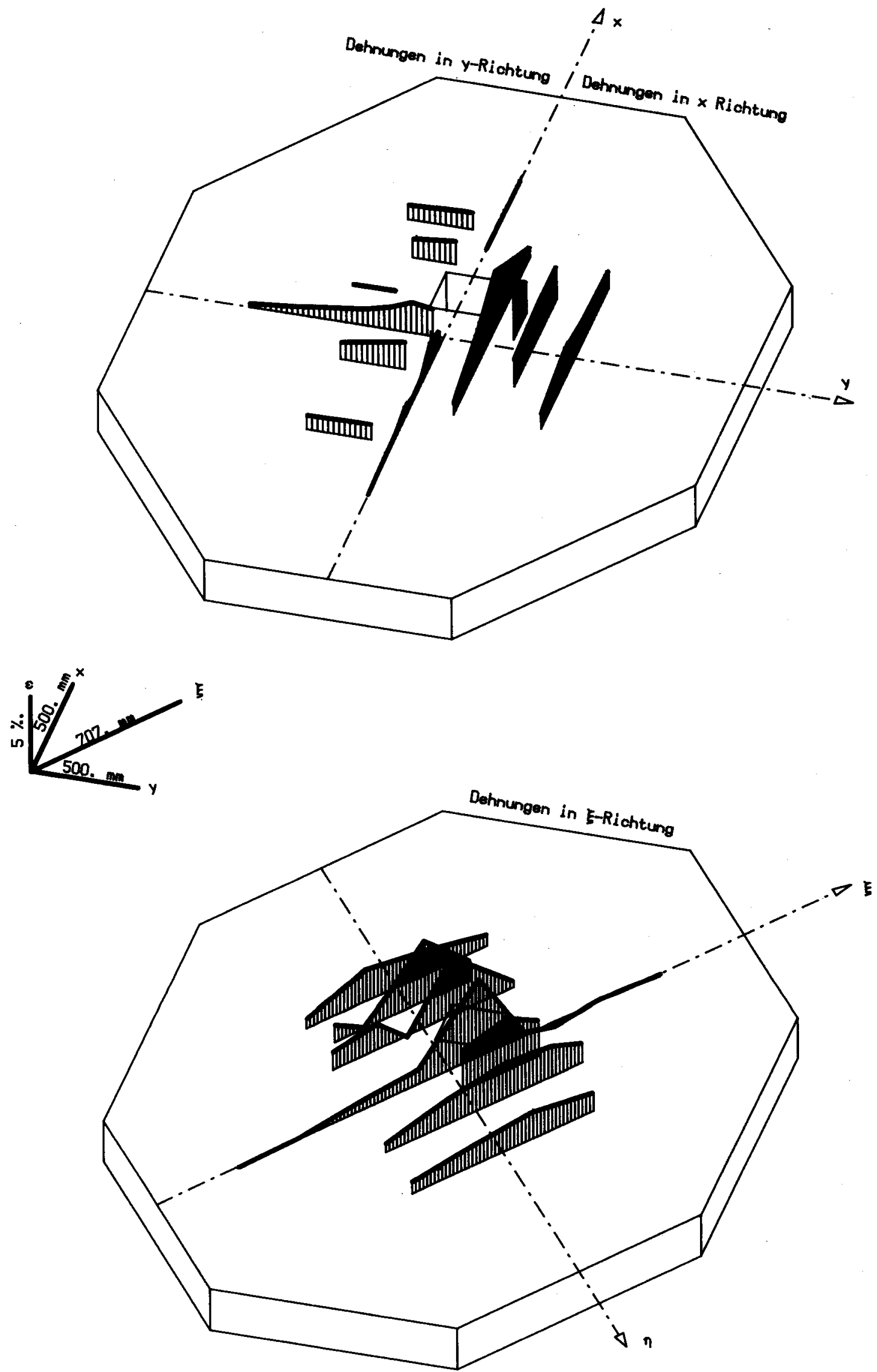

Bild 44: Mittlere Dehnungen der oberen Messebene, Platte P 17, Laststufe 9 

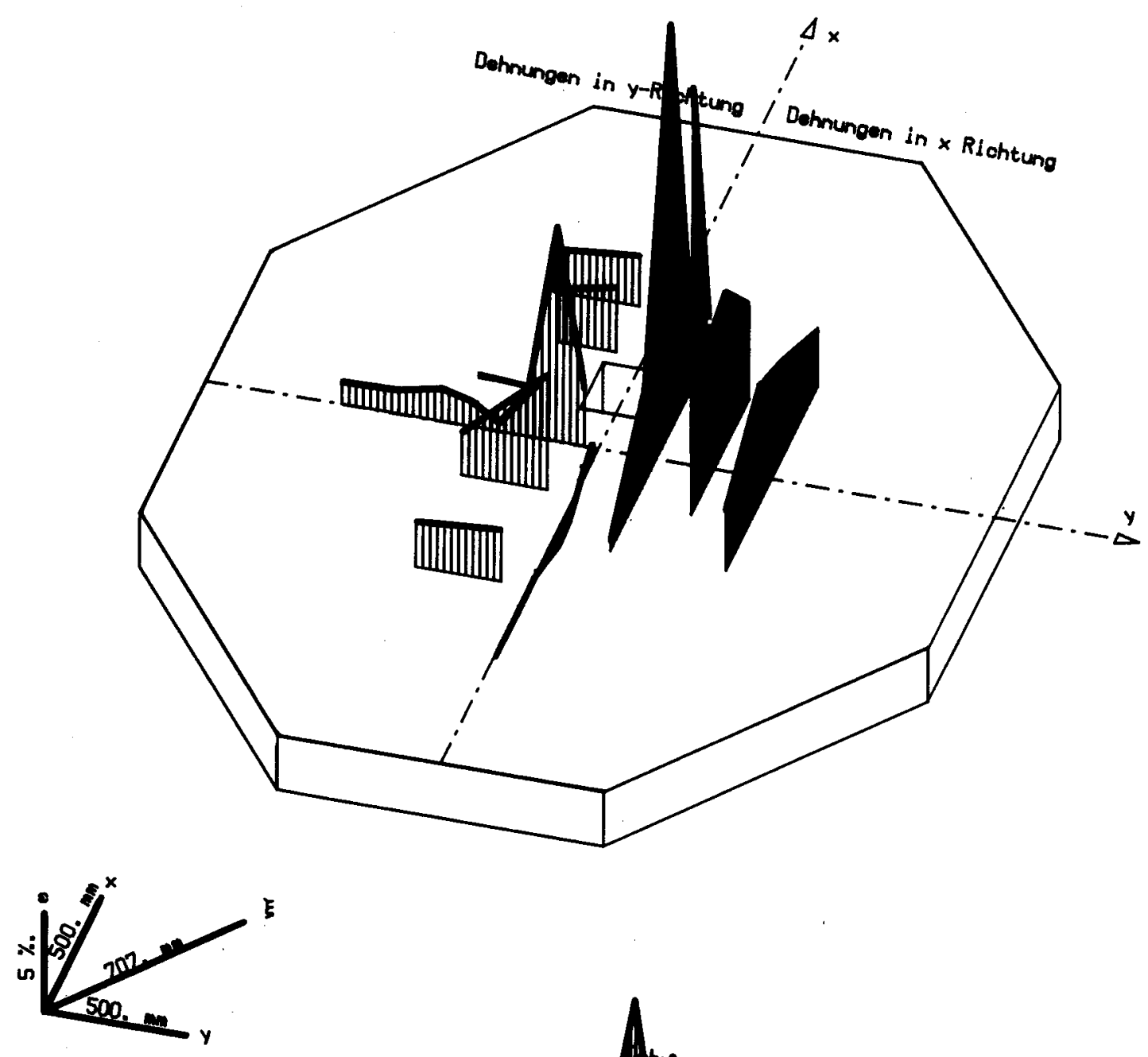

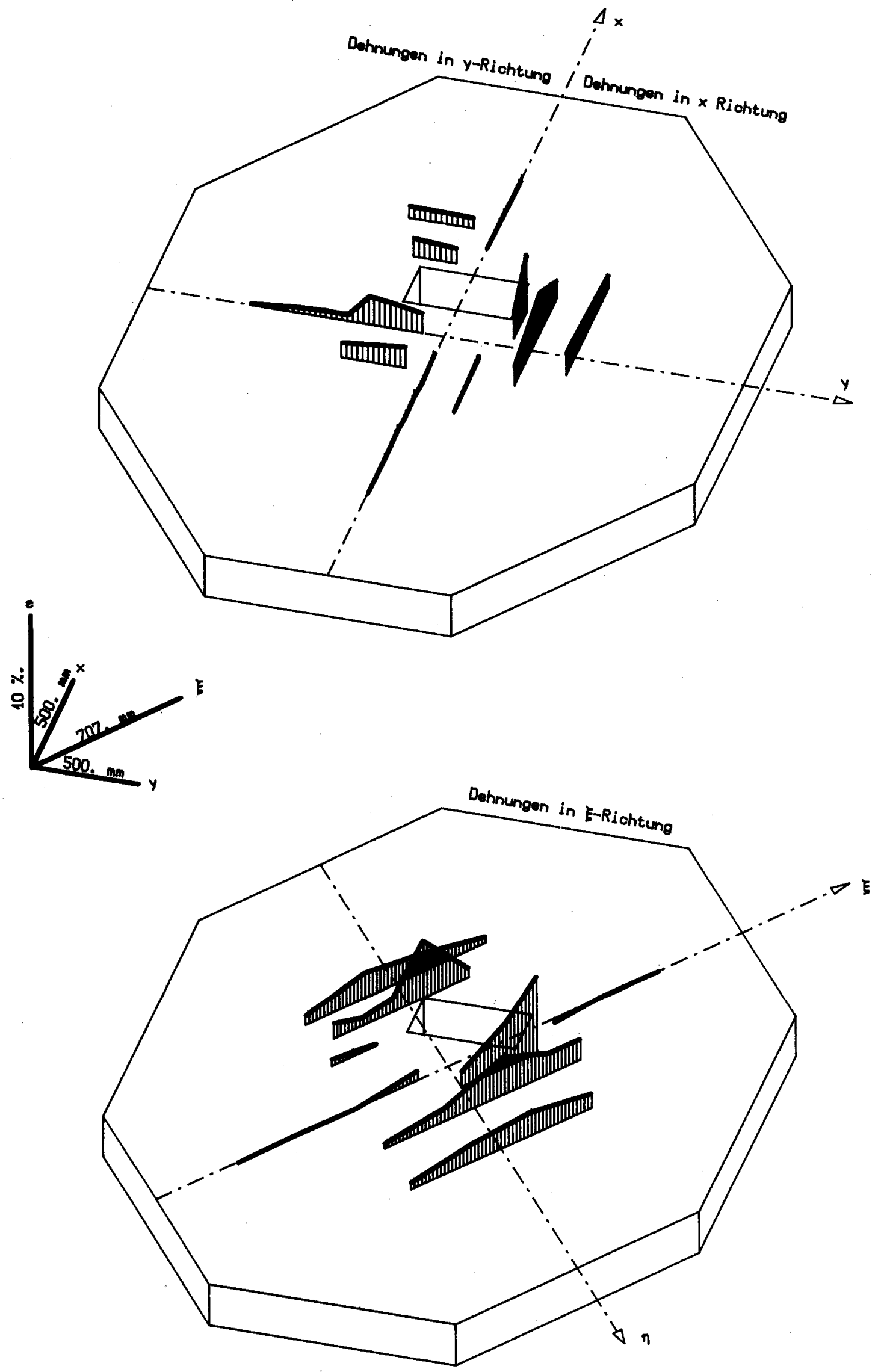

B1ld 46: Mittlere Dehnungen der oberen Messebene, Platte P 18, Laststufe 9 

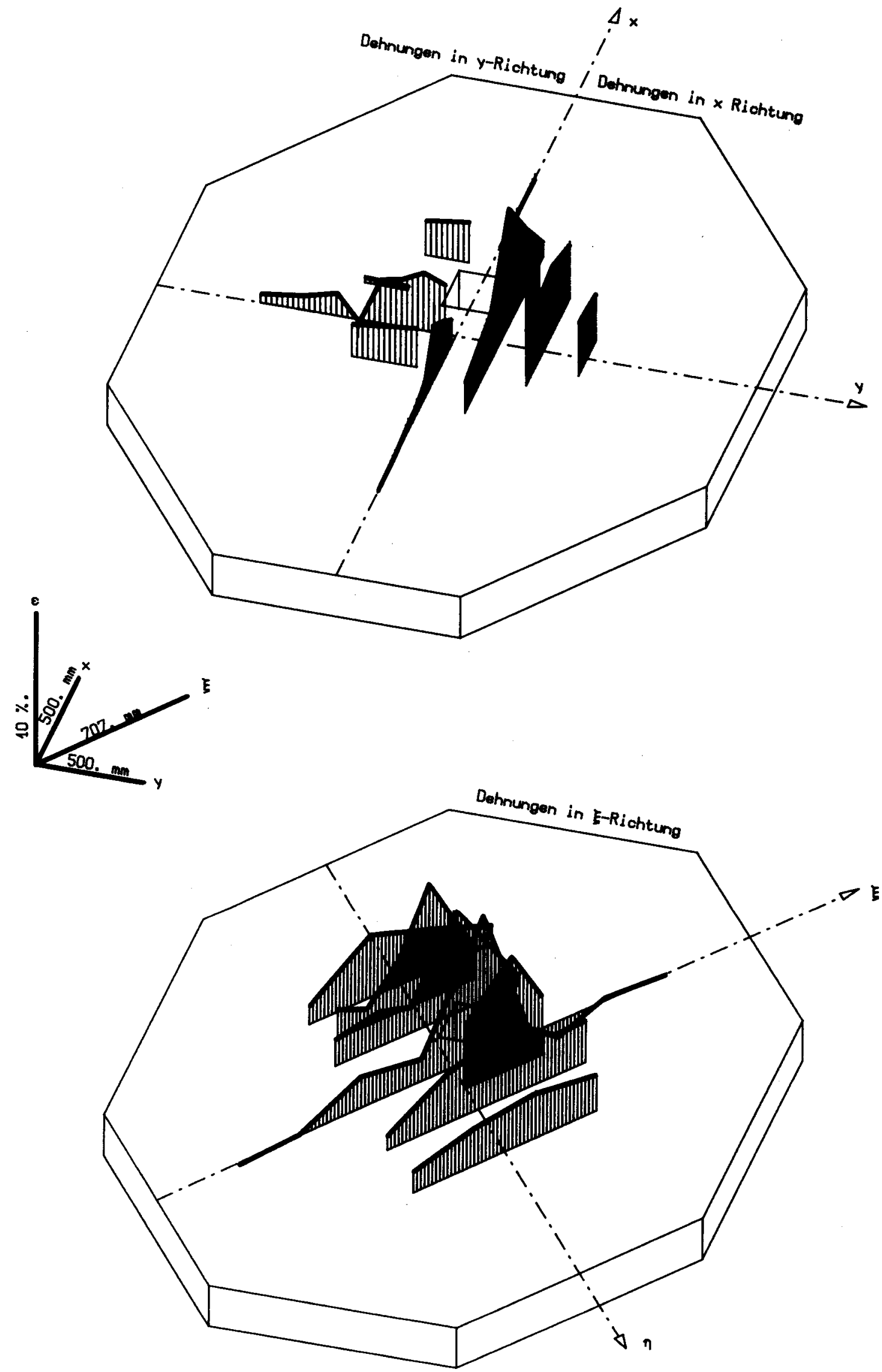

Bild 47: Mittlere Dehnungen der oberen Messebene, Platte P 19, Laststufe 12 

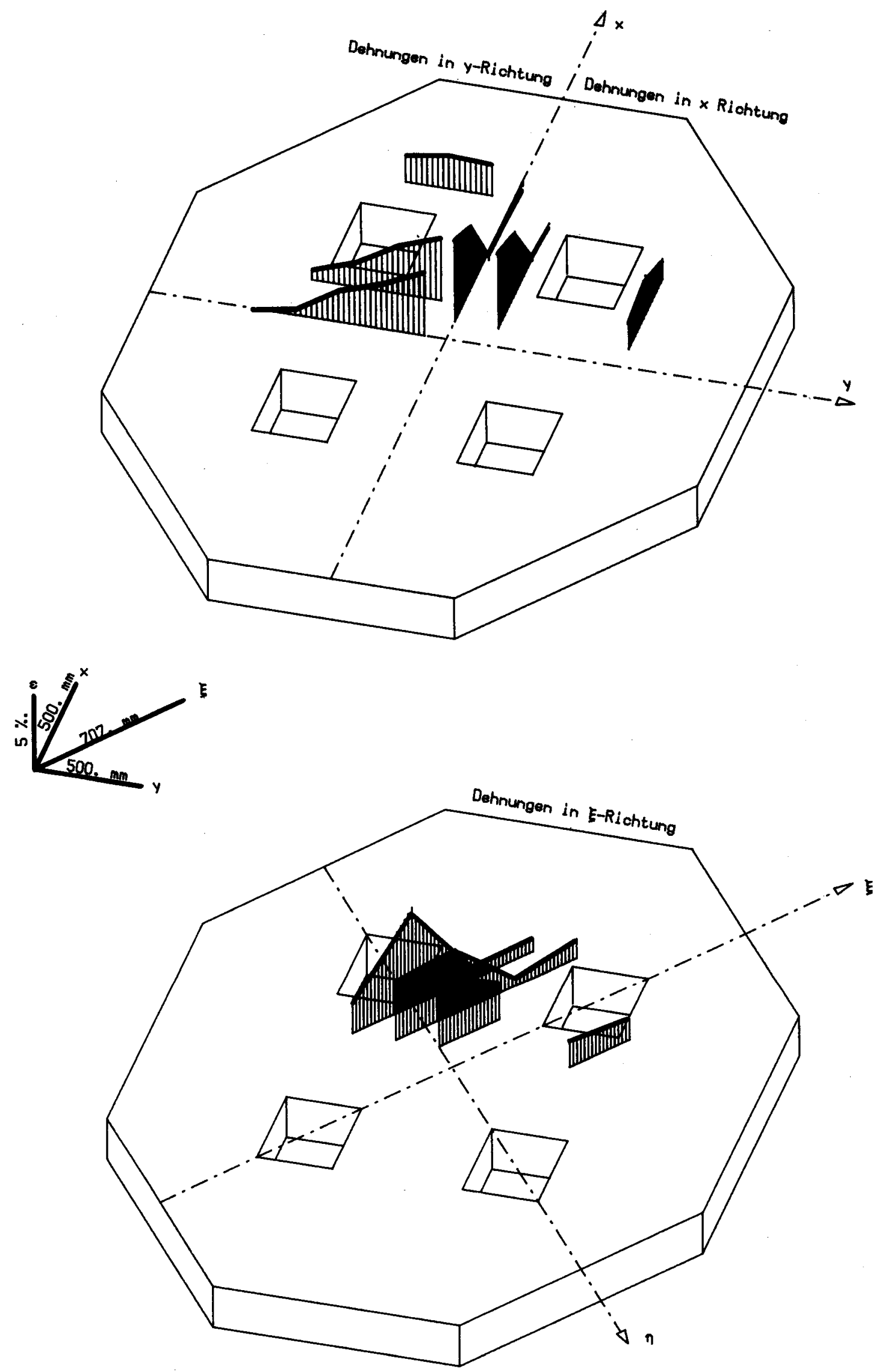

Bild 48: Mittlere Dehnungen der oberen Messebene, Platte P 20, Laststufe 26 


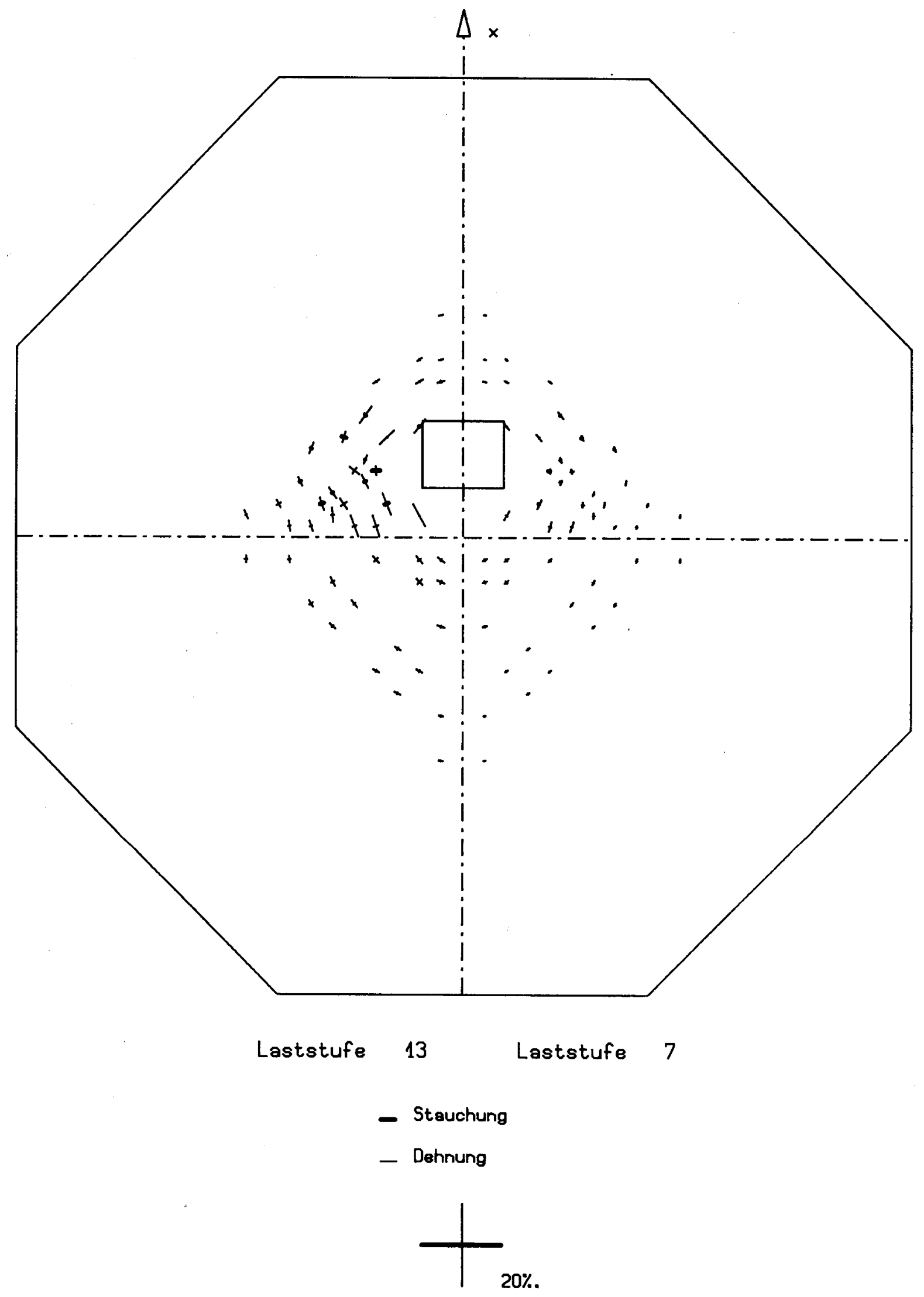

B1ld 49: Hauptdehnungen der oberen Messebene, Platte P 16 


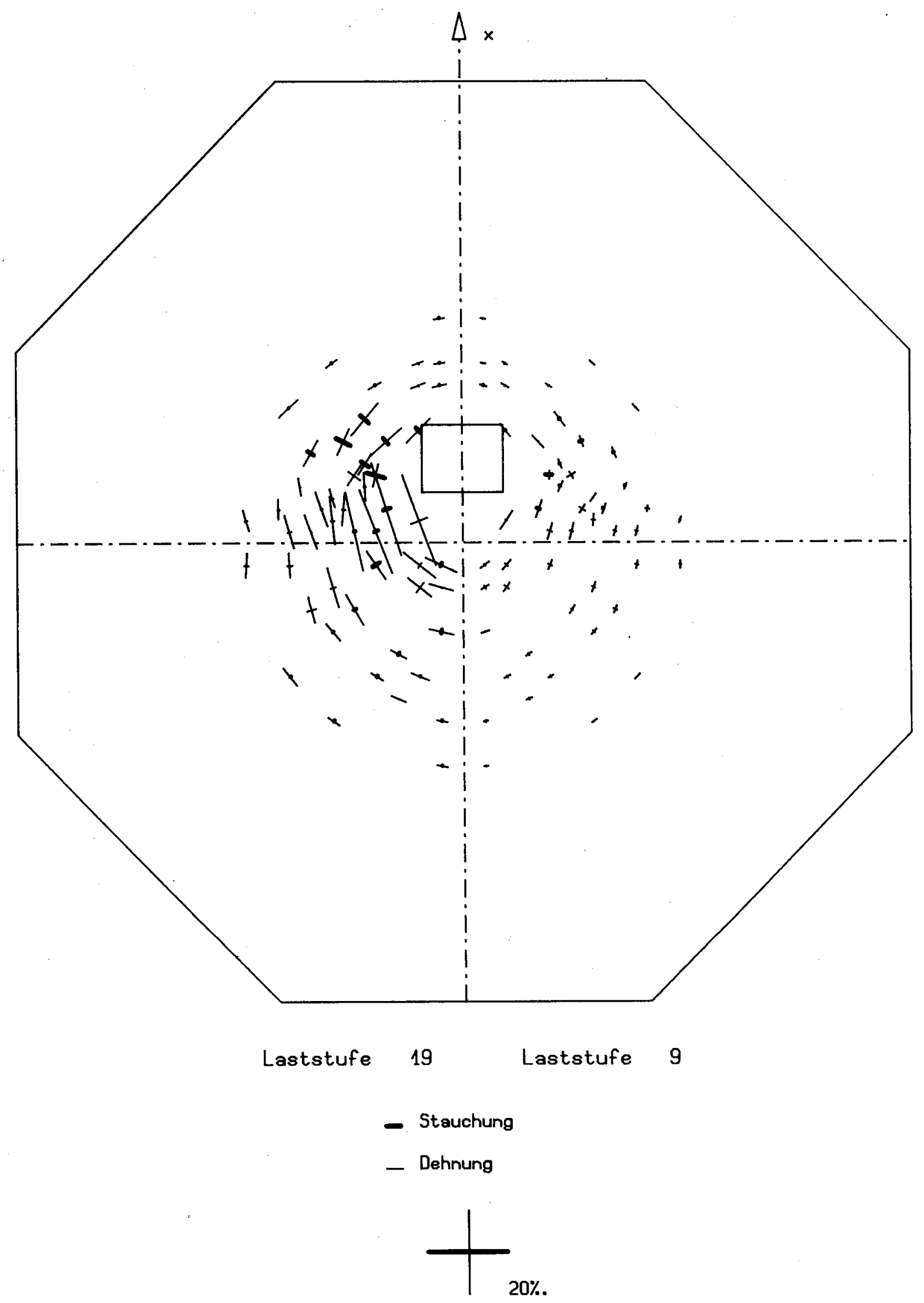

Bild 50: Hauptdehnungen der oberen Messebene, Platte P 17 


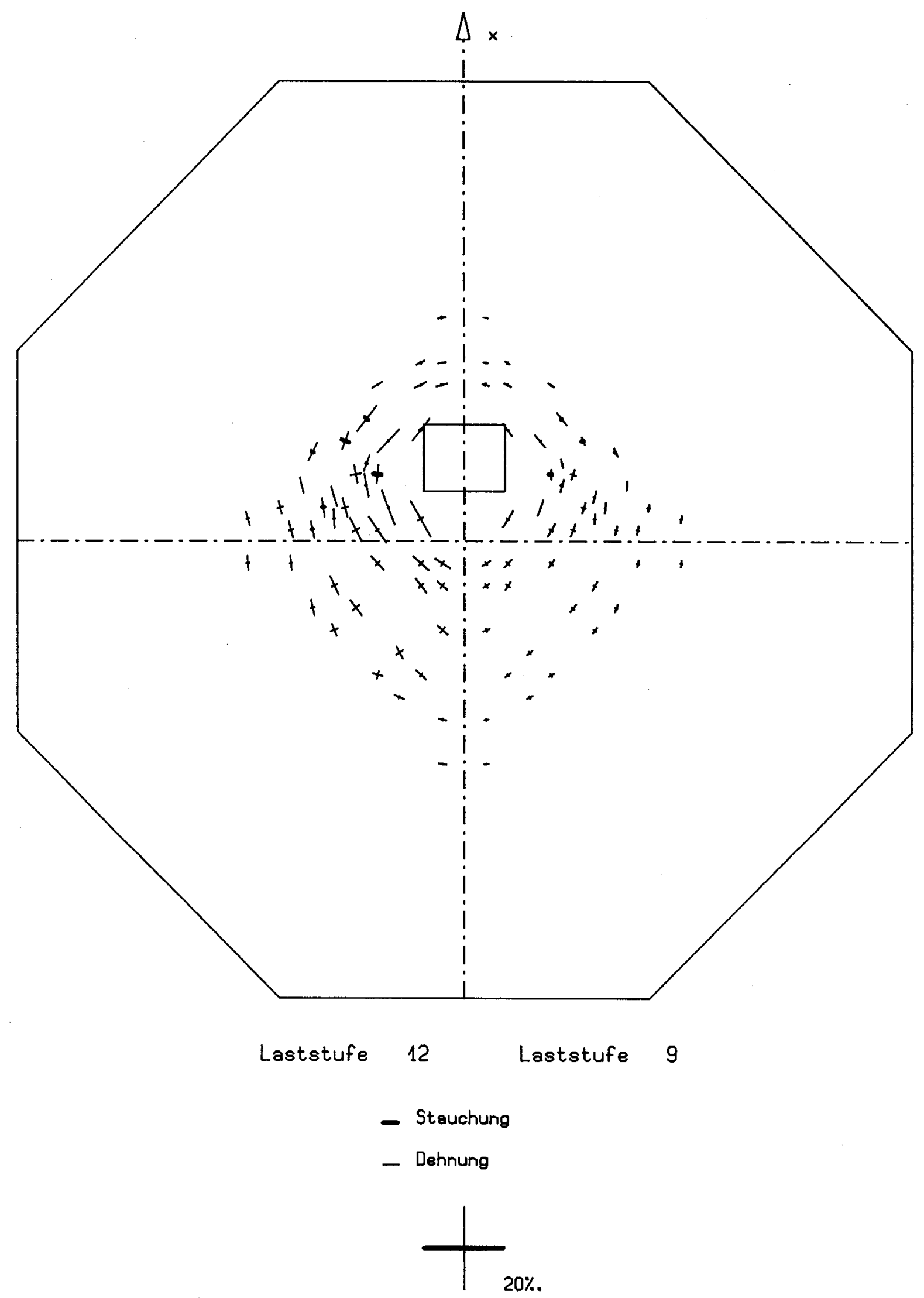

Bild 51: Hauptdehnungen der oberen Messebene, Platte P 19 


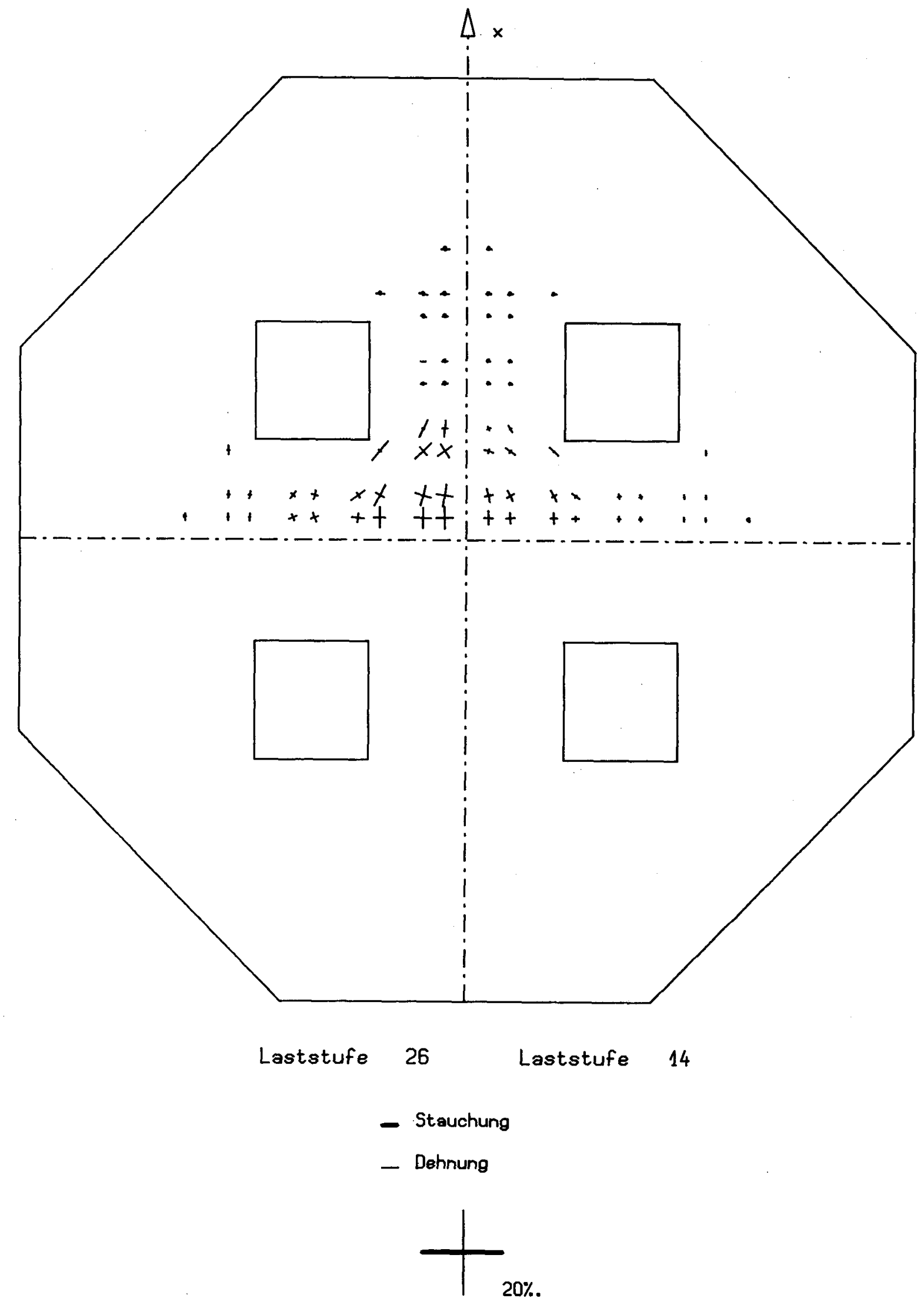

Bild 52: Hauptdehnungen der oberen Messebene, Platte P 20 


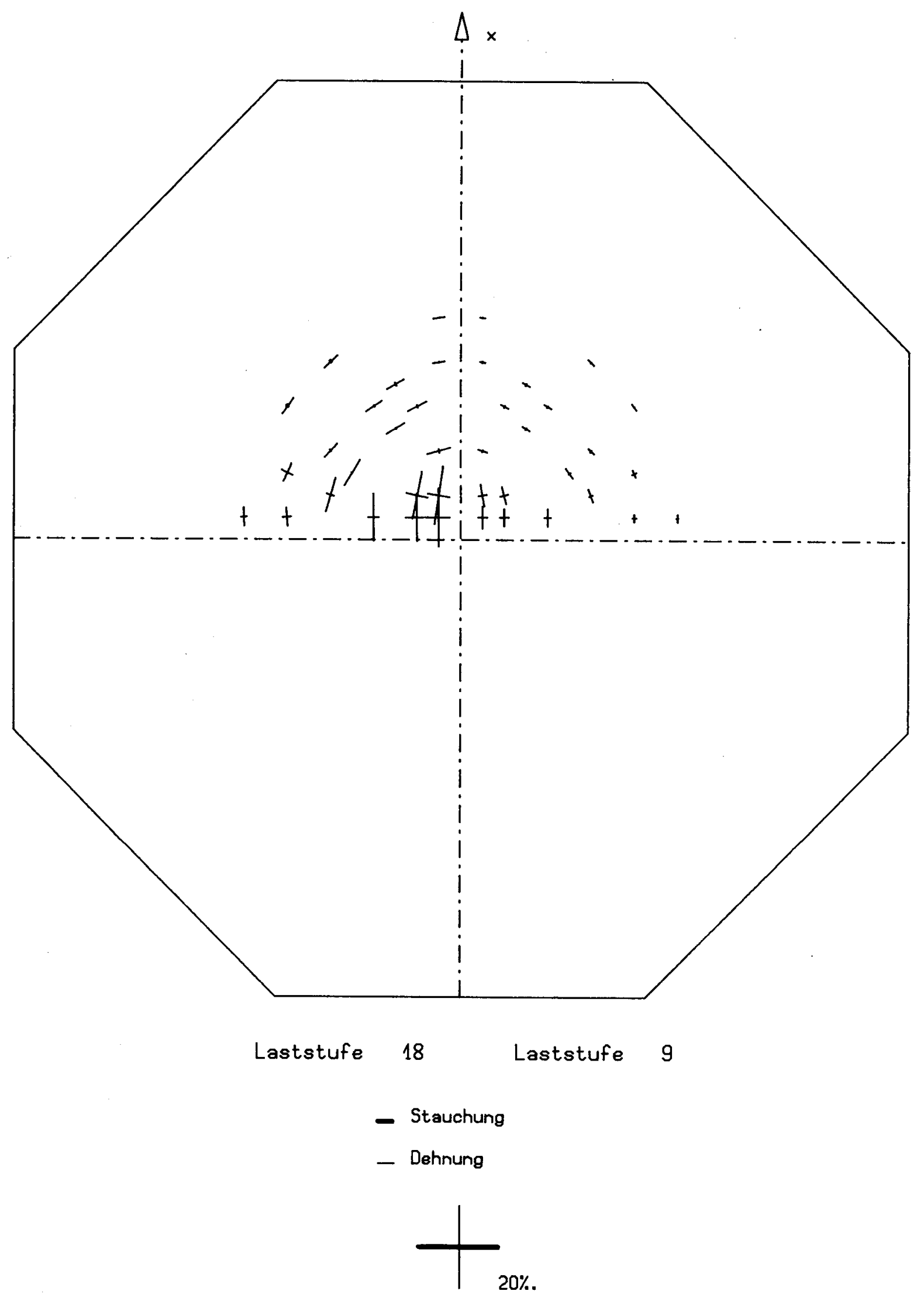

Bild 53: Hauptdehnungen der oberen Messebene, Platte P 22 


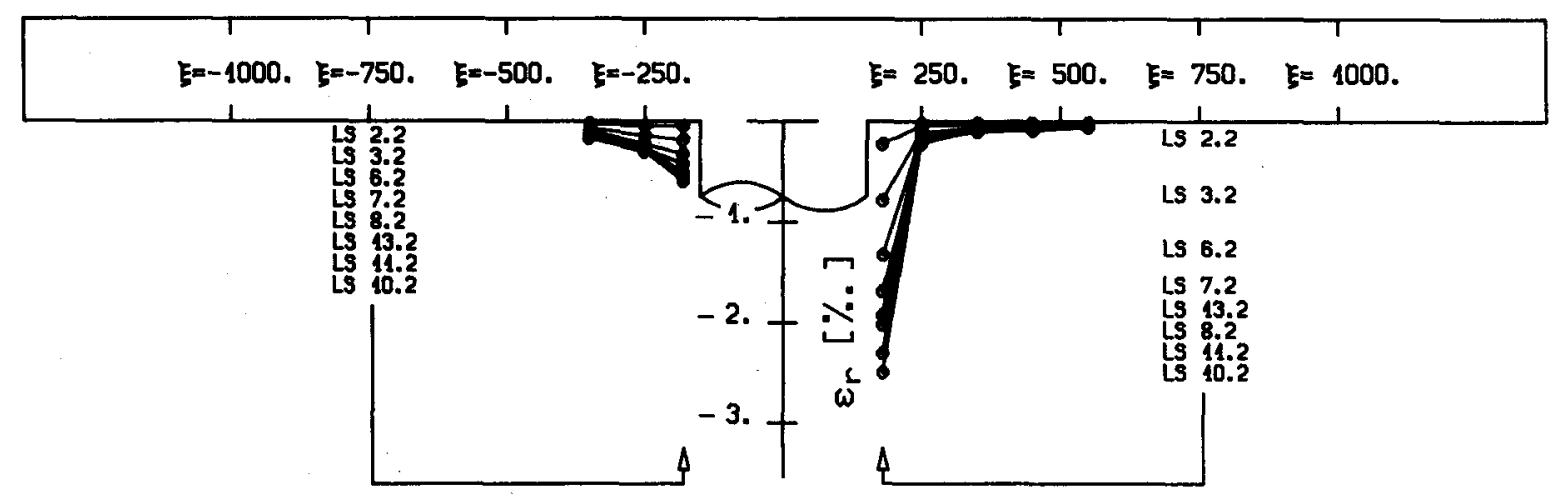

Radiale Betonstauchungen auf der E-Rchse

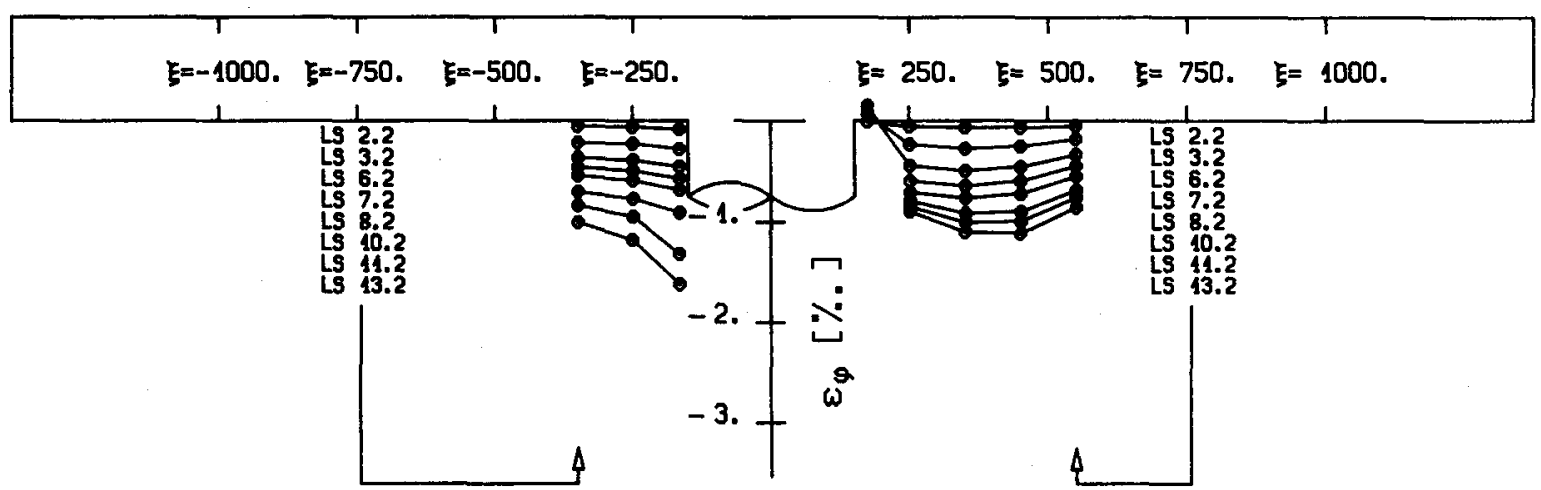

Tangentiale Betonstauchungen auf der $\xi$-Achse
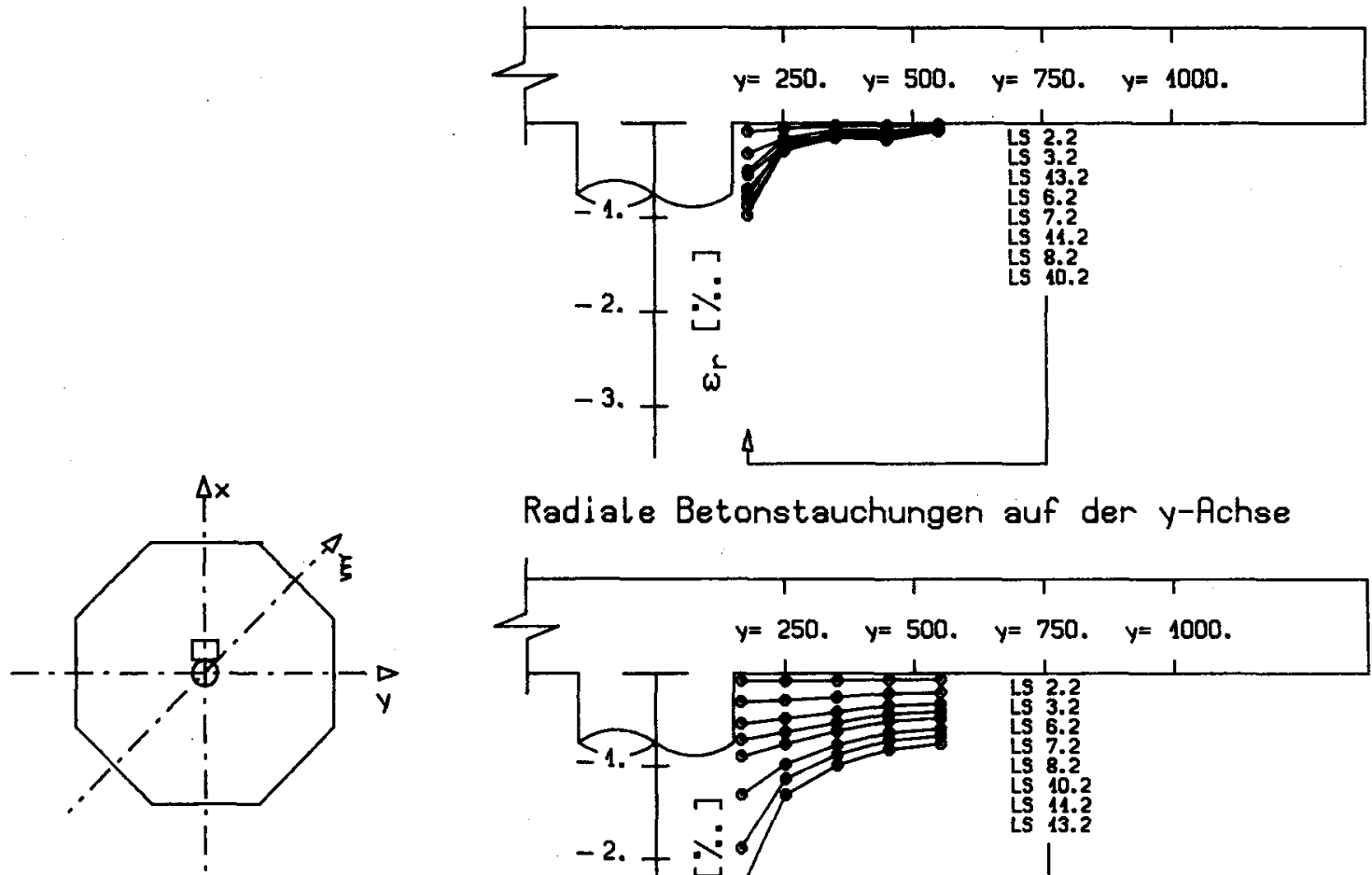

Radiale Betonstauchungen auf der y-Achse

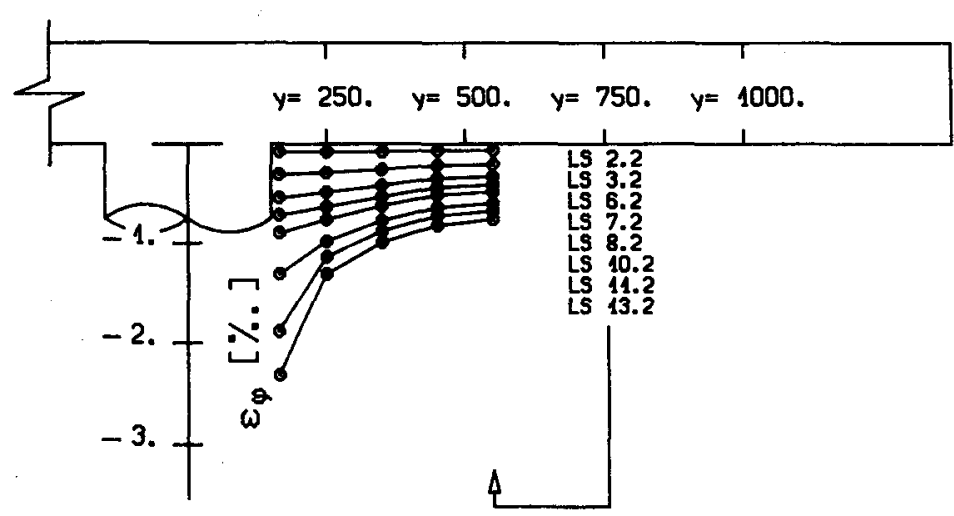

Tangentiale Betonstauchungen auf der y-Achse

B1ld 54: Betonstauchungen der Plattenunterseite, Platte $P 16$ 


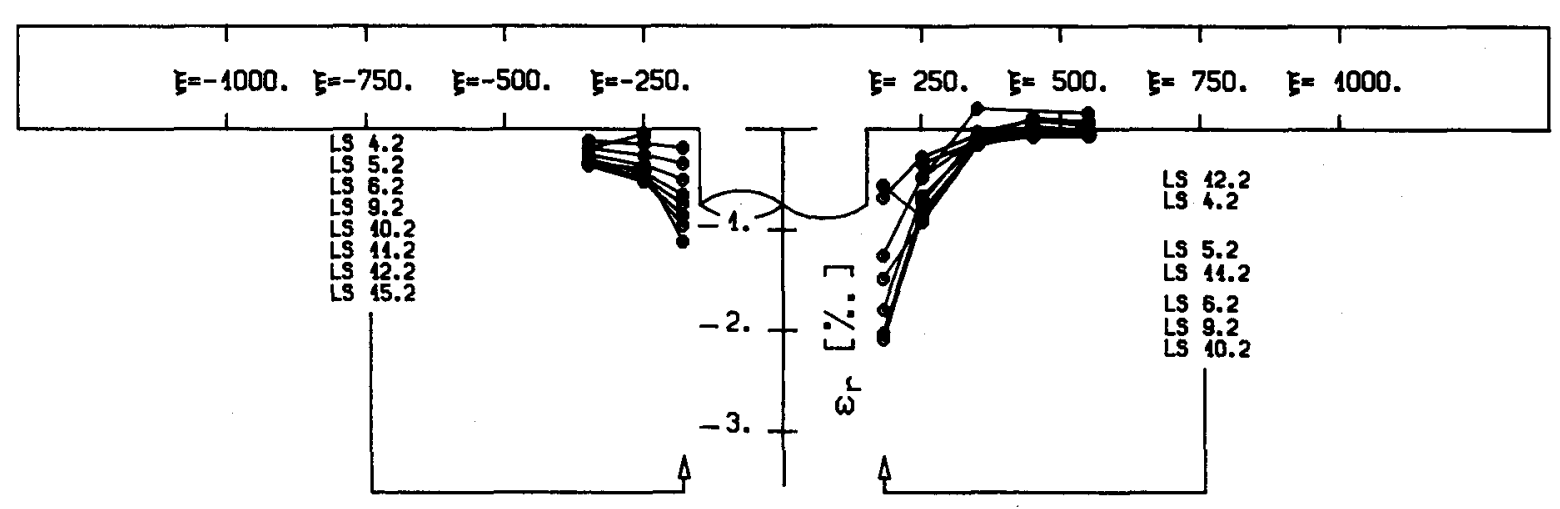

Radiale Betonstauchungen auf der E-Achse

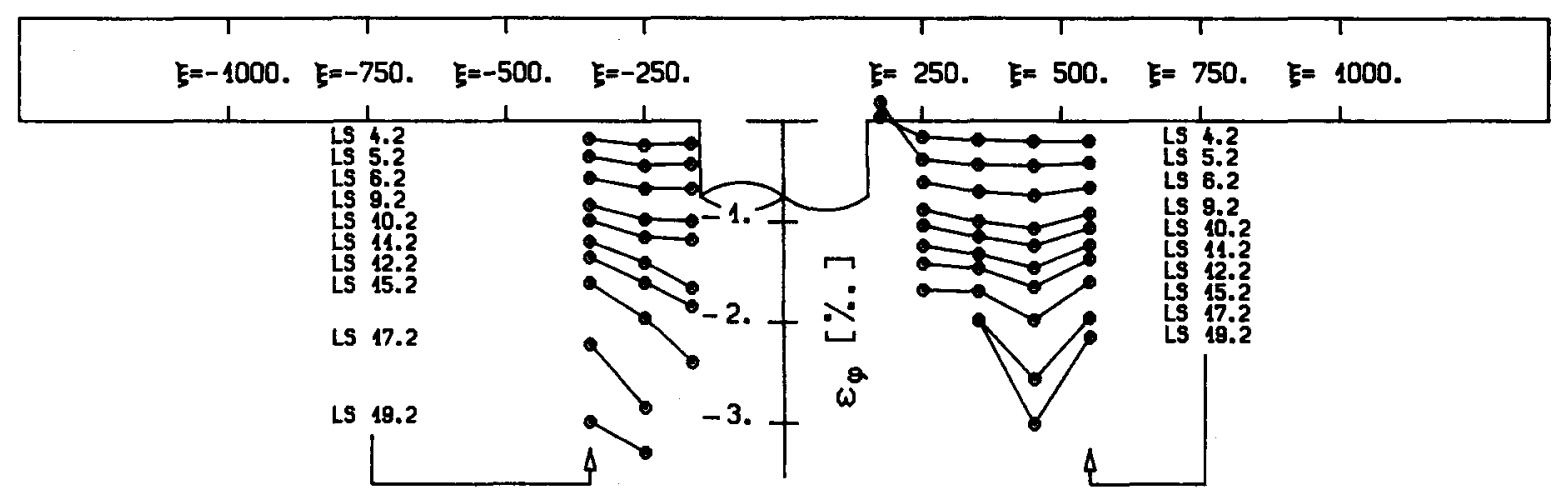

Tangentiale Betonstauchungen auf der E-Achse
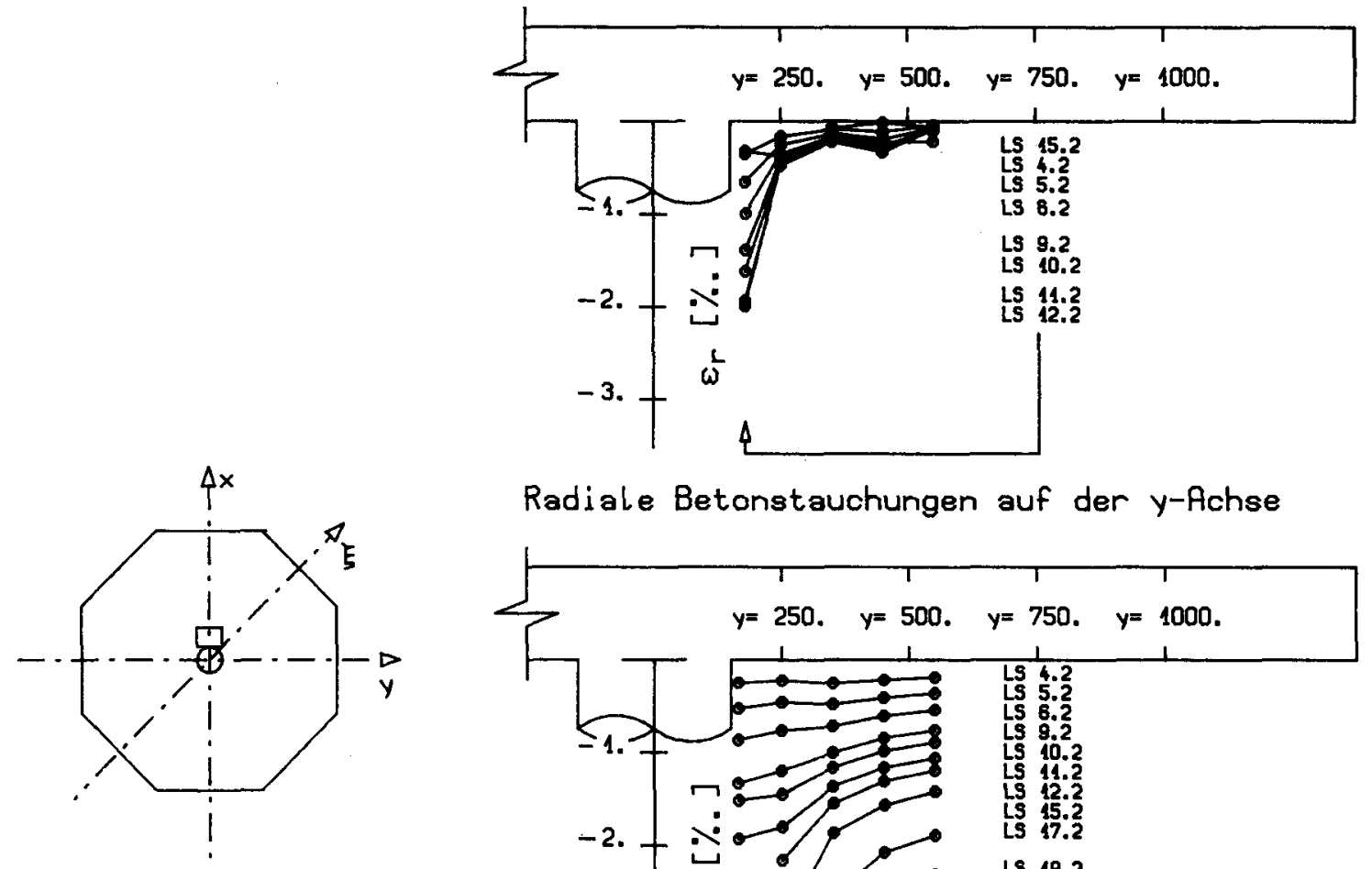

Radiale Betonstauchungen auf der $y$-Achse

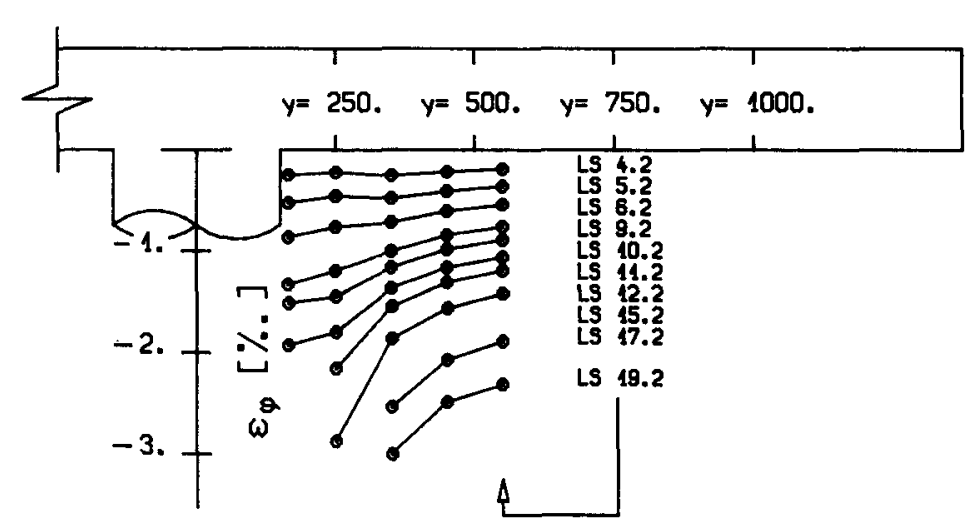

Tangentiale Betonstauchungen auf der $y$-Achse 

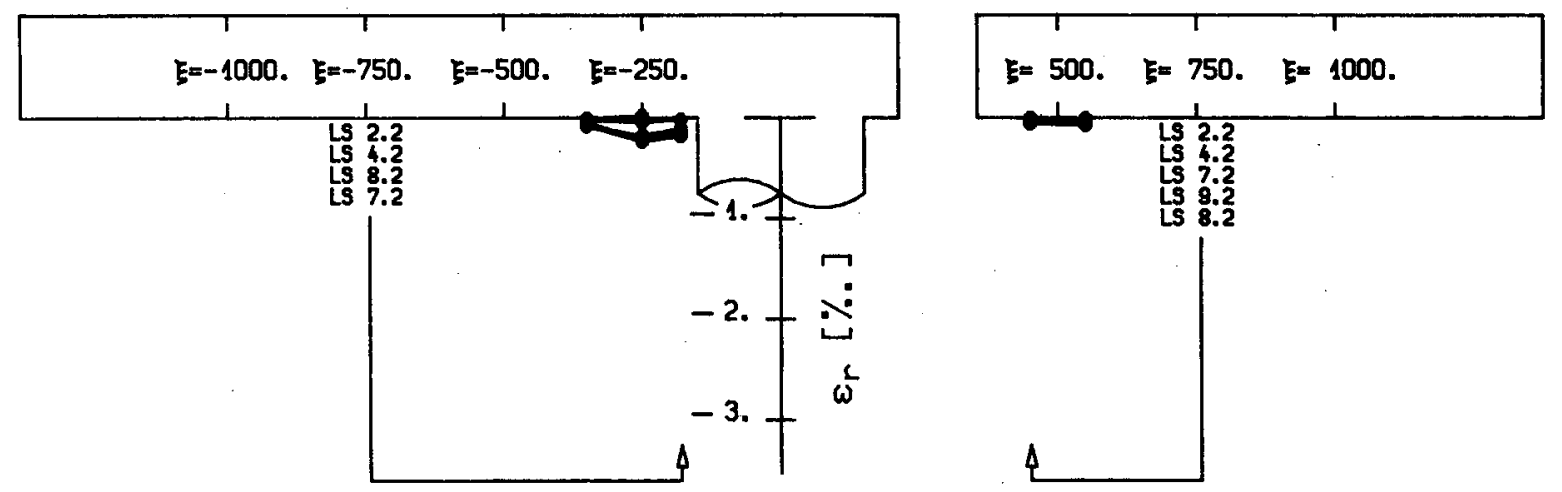

Radiale Betonstauchungen auf der E-Achse
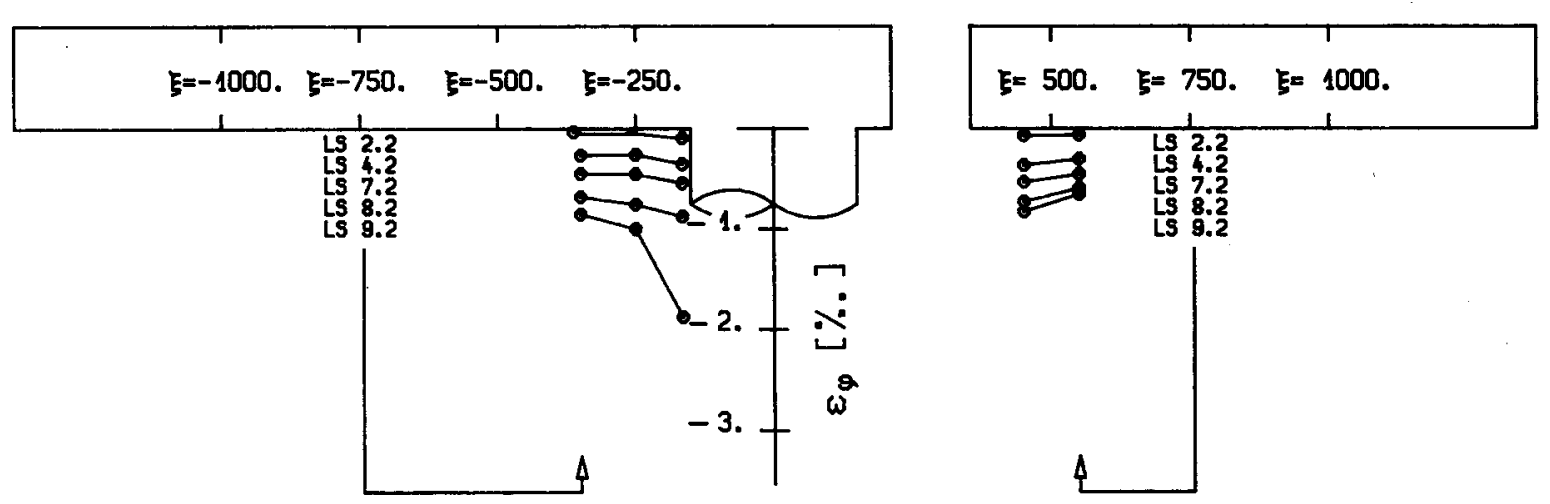

Tangentiale Betonstauchungen auf der E-Achse
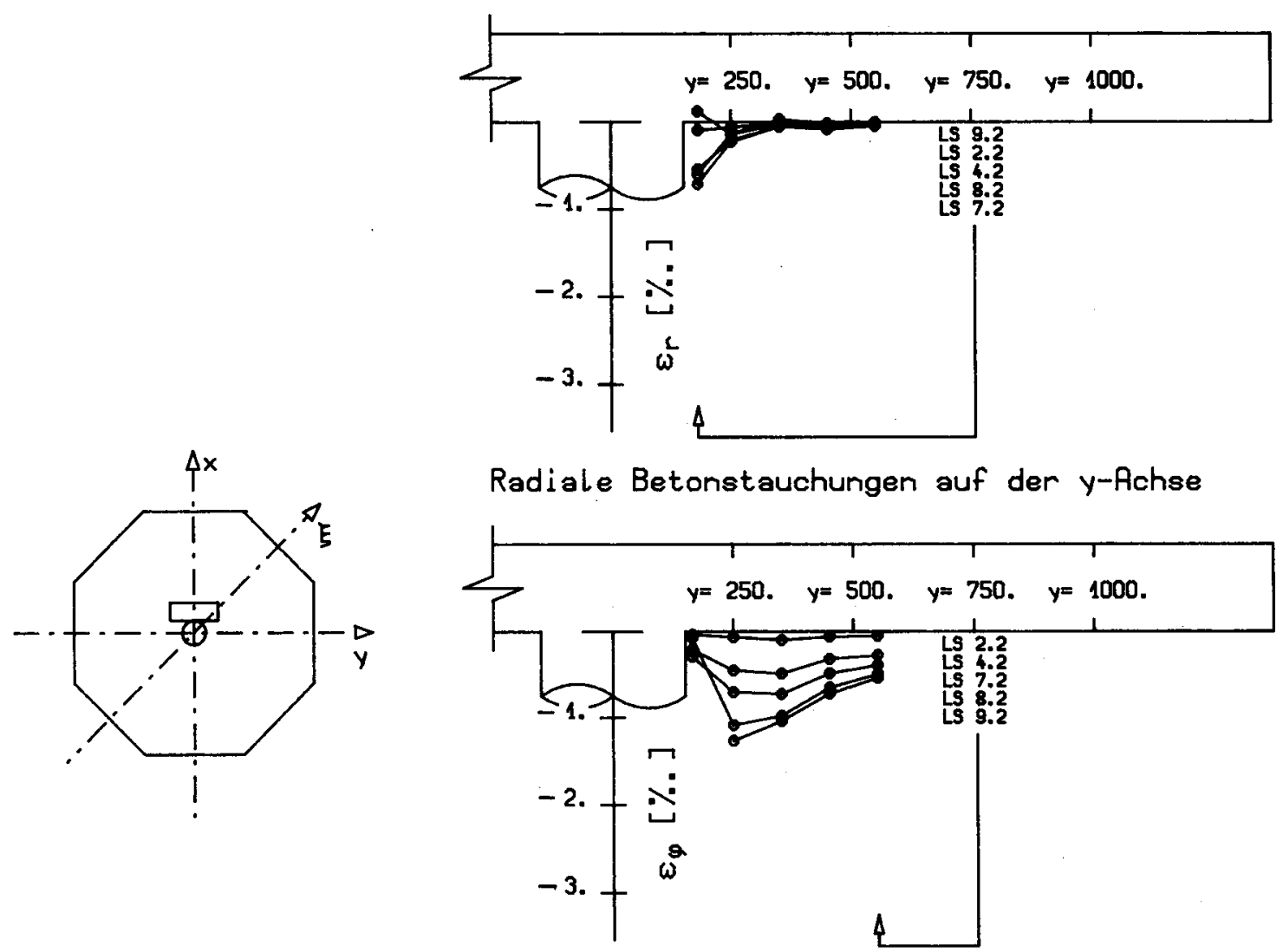

Tangentiale Betonstauchungen auf der y-Achse 


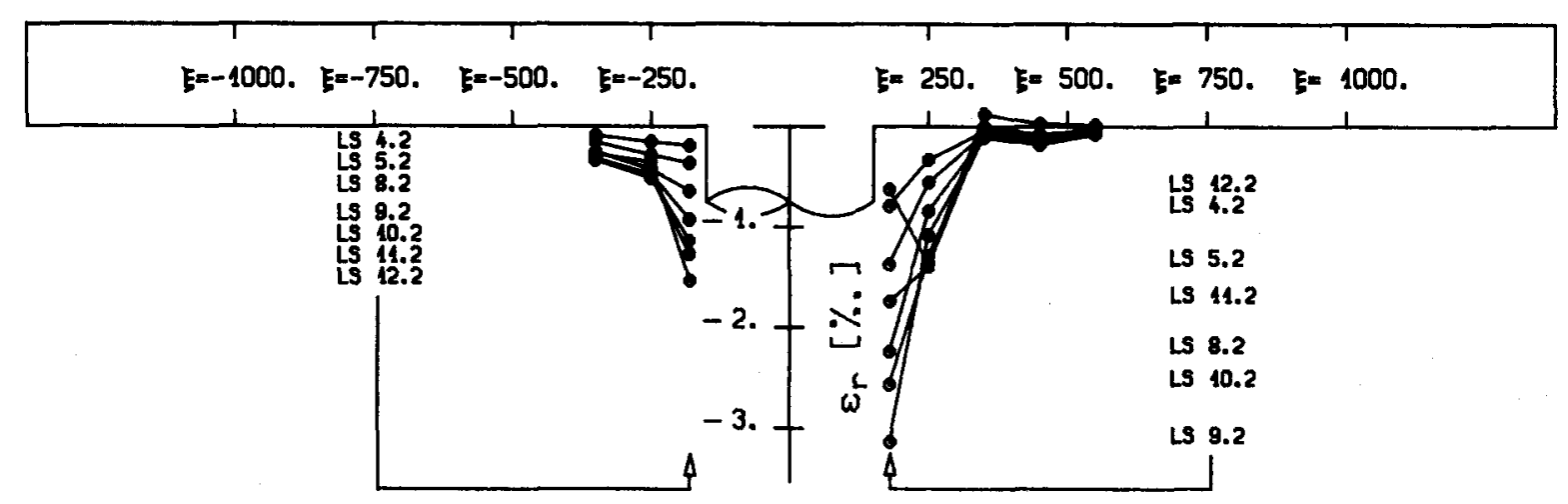

Radiale Betonstauchungen auf der E-Achse

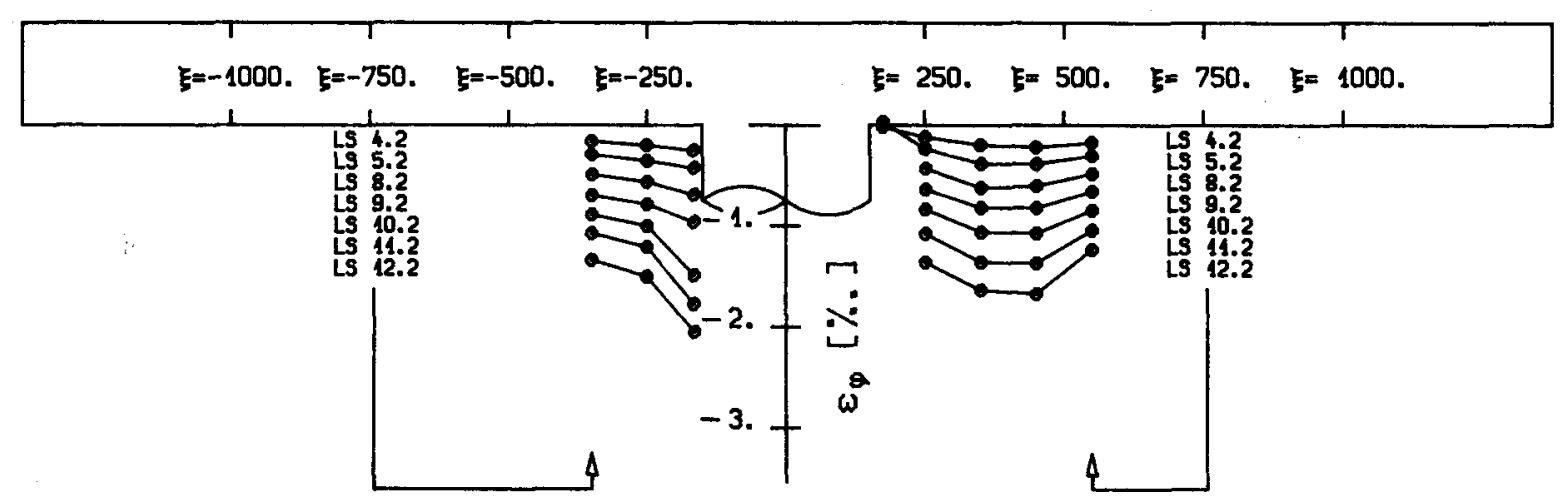

Tangentiale Betonstauchungen auf der $\xi$-Achse
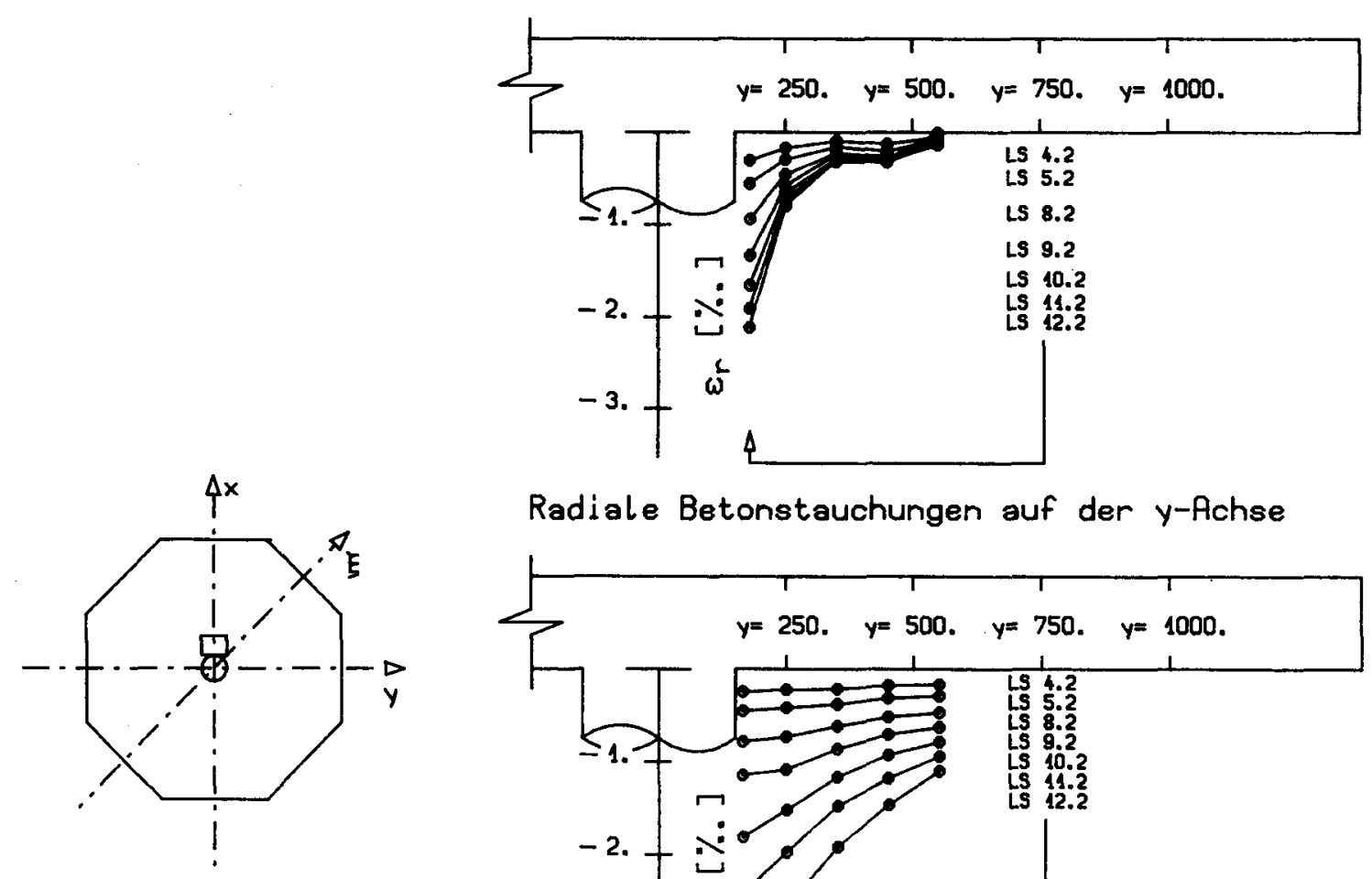

Radiale Betonstauchungen auf der $y$-Achse

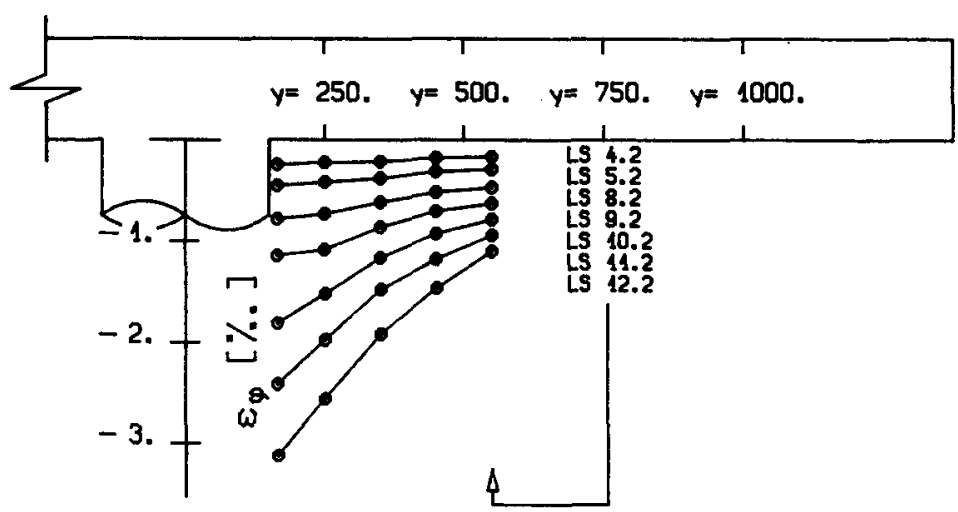

Tangentiale Betonstauchungen auf der $y$-Achse 


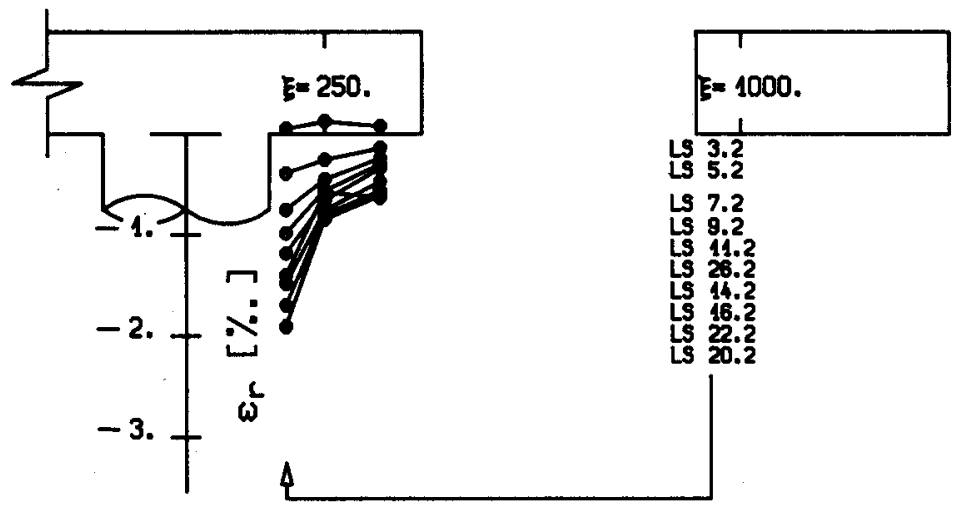

Radiale Betonstauchungen auf der E-Achse

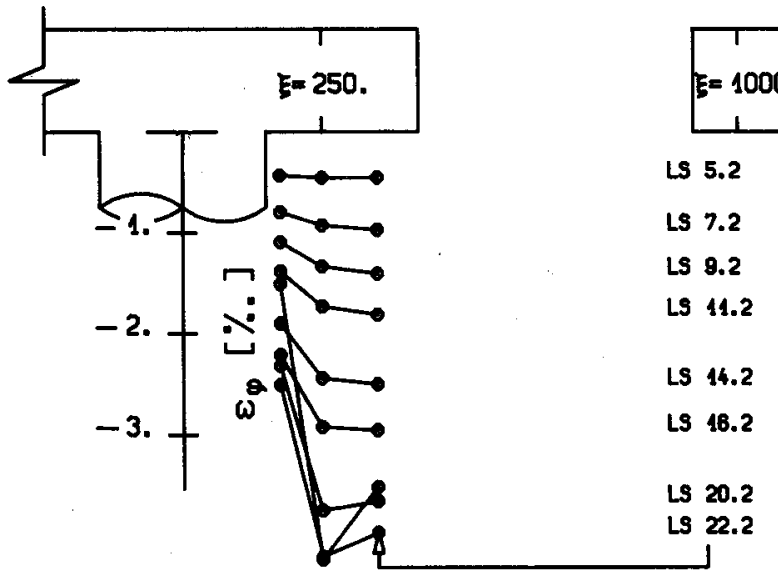

Tangentiale Betonstauchungen auf der E-Achse

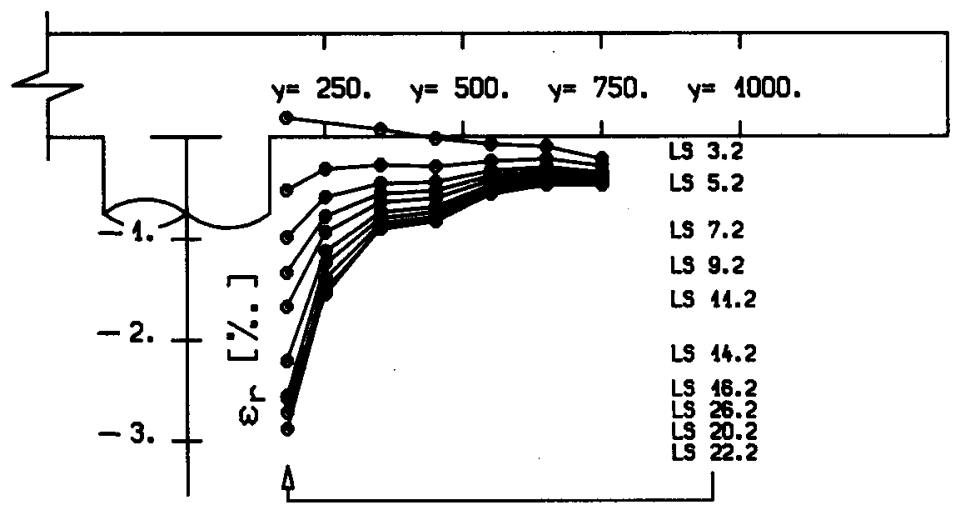

Radiale Betonstauchungen auf der $y$-Achse

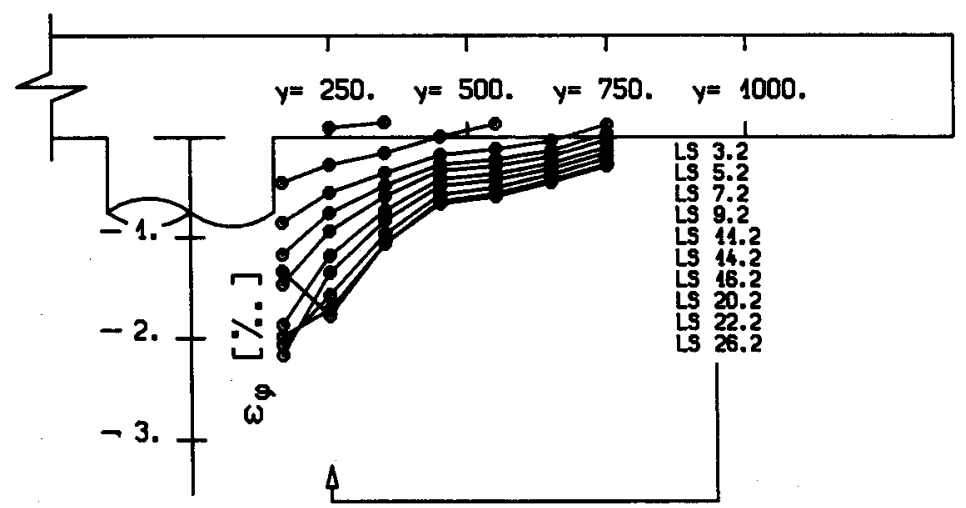

Tangentiale Betonstauchungen auf der $y$-Achse 

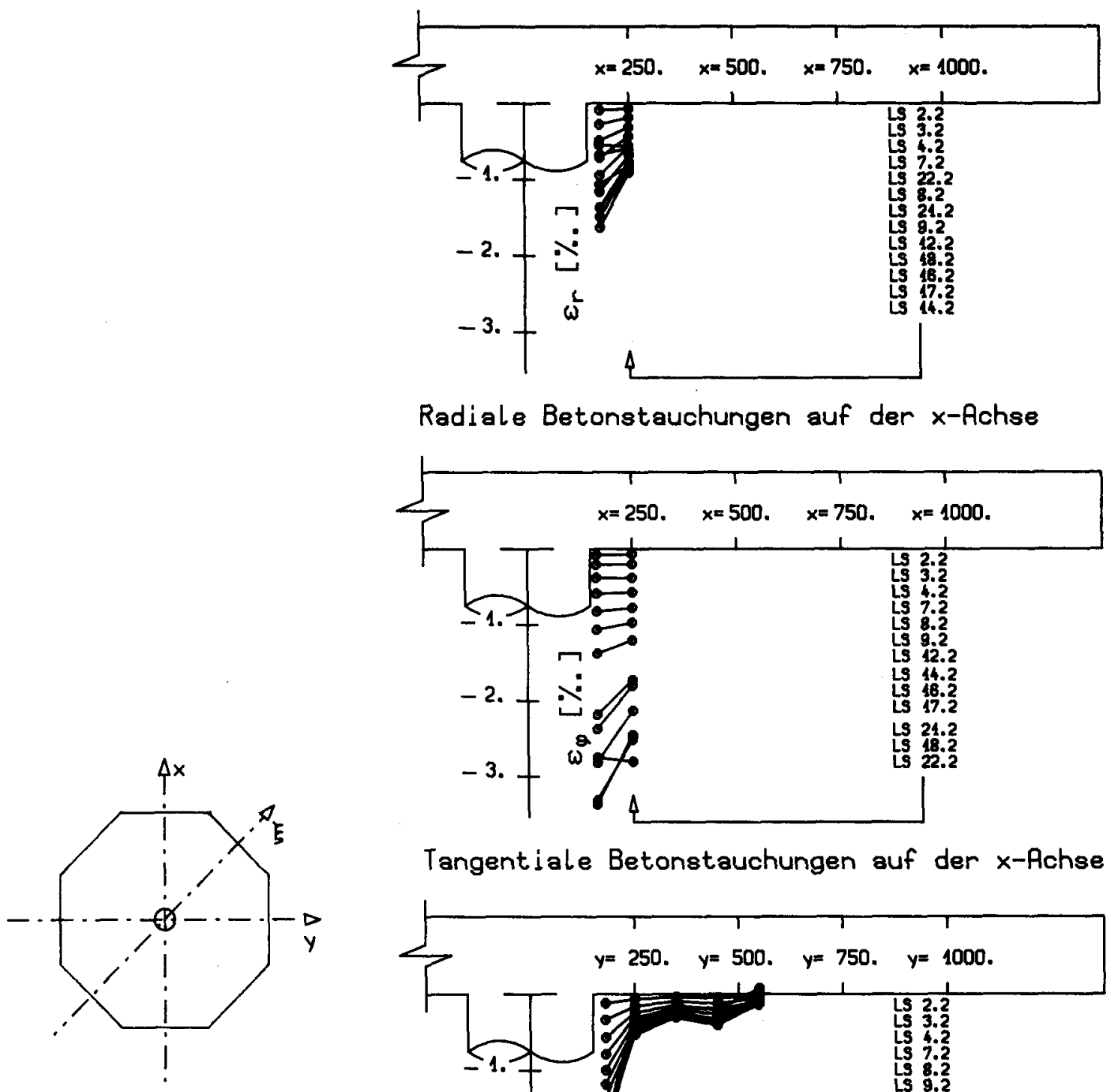

Radiale Betonstauchungen auf der $x$-Achse

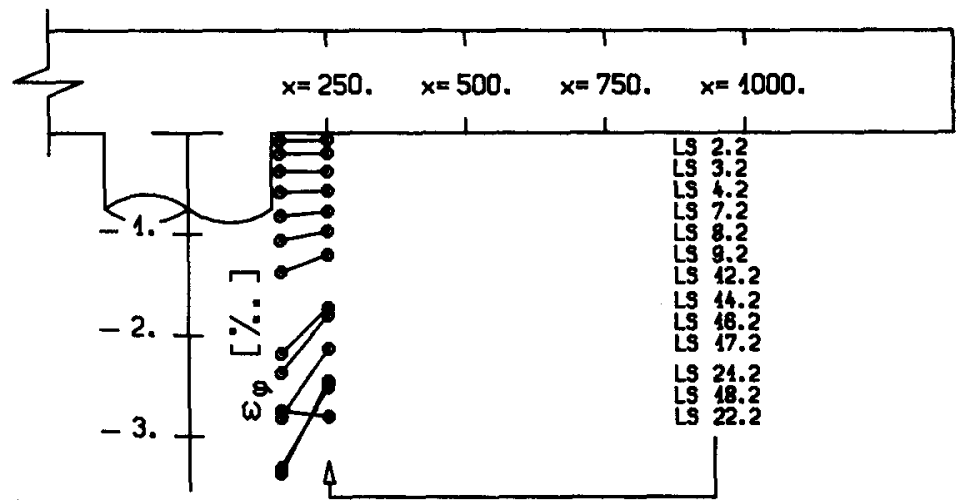

Tangentiale Betonstauchungen auf der $x$-Achse

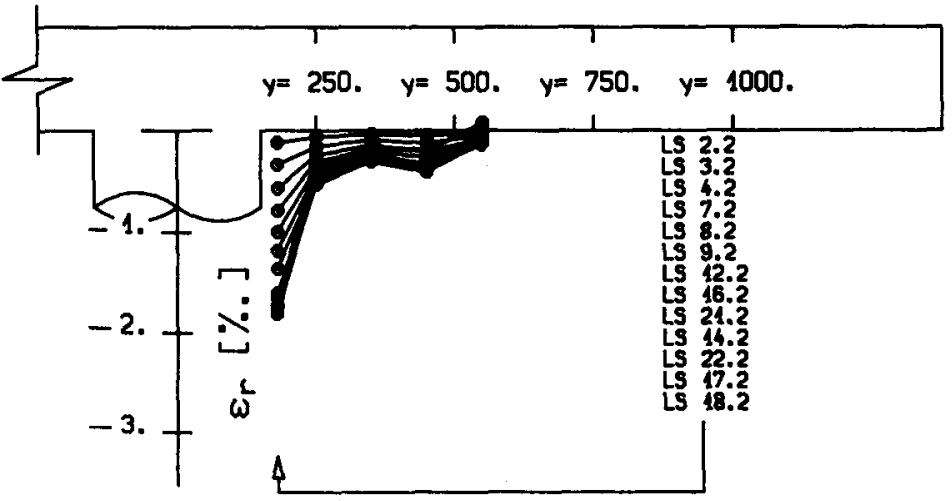

Radiale Betonstauchungen auf der y-Achse

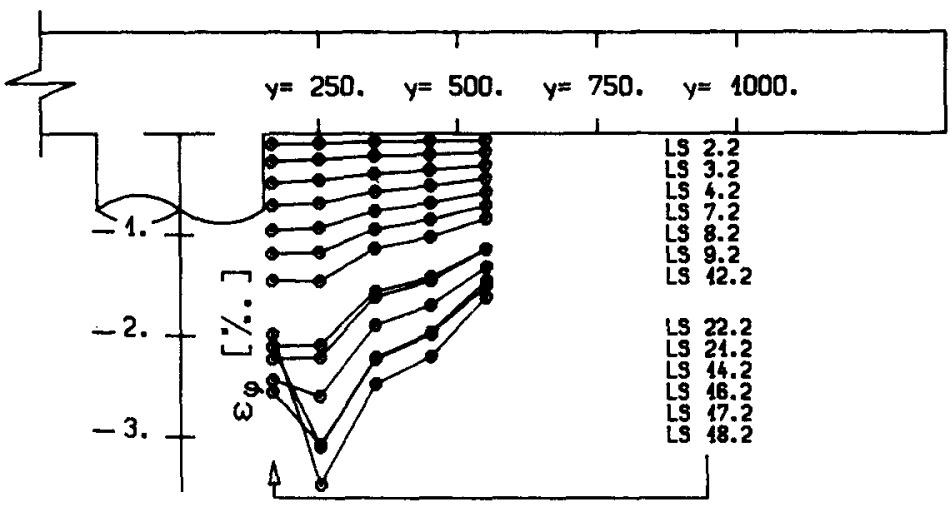

Tangentiale Betonstauchungen auf der $y$-Achse

Bild 59: Betonstauchungen der Plattenunterselte, Platte P 22 

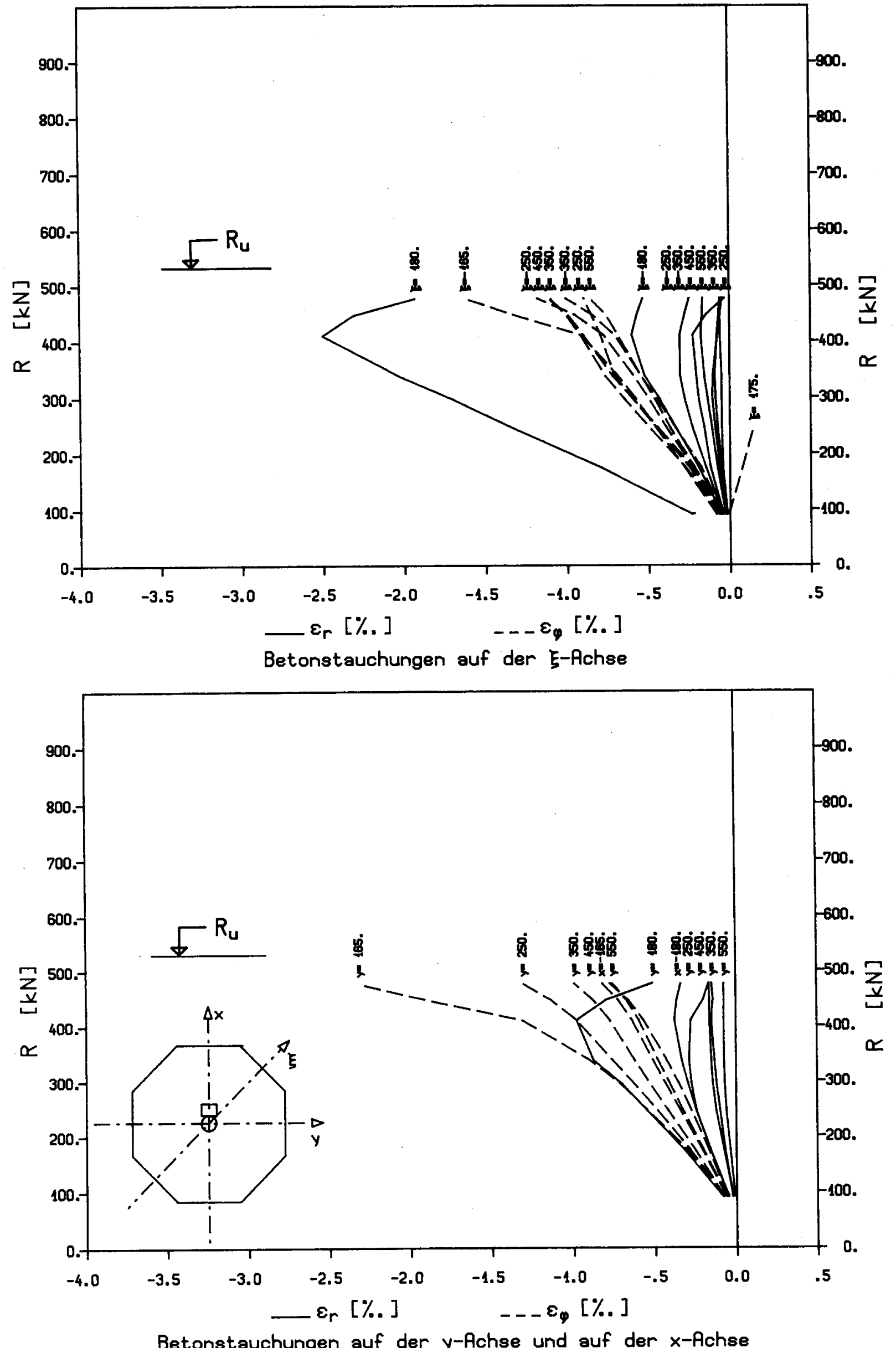

B1ld 60: Betonstauchungen der Plattenunterseite, messstellenwelse, Platte P 16 

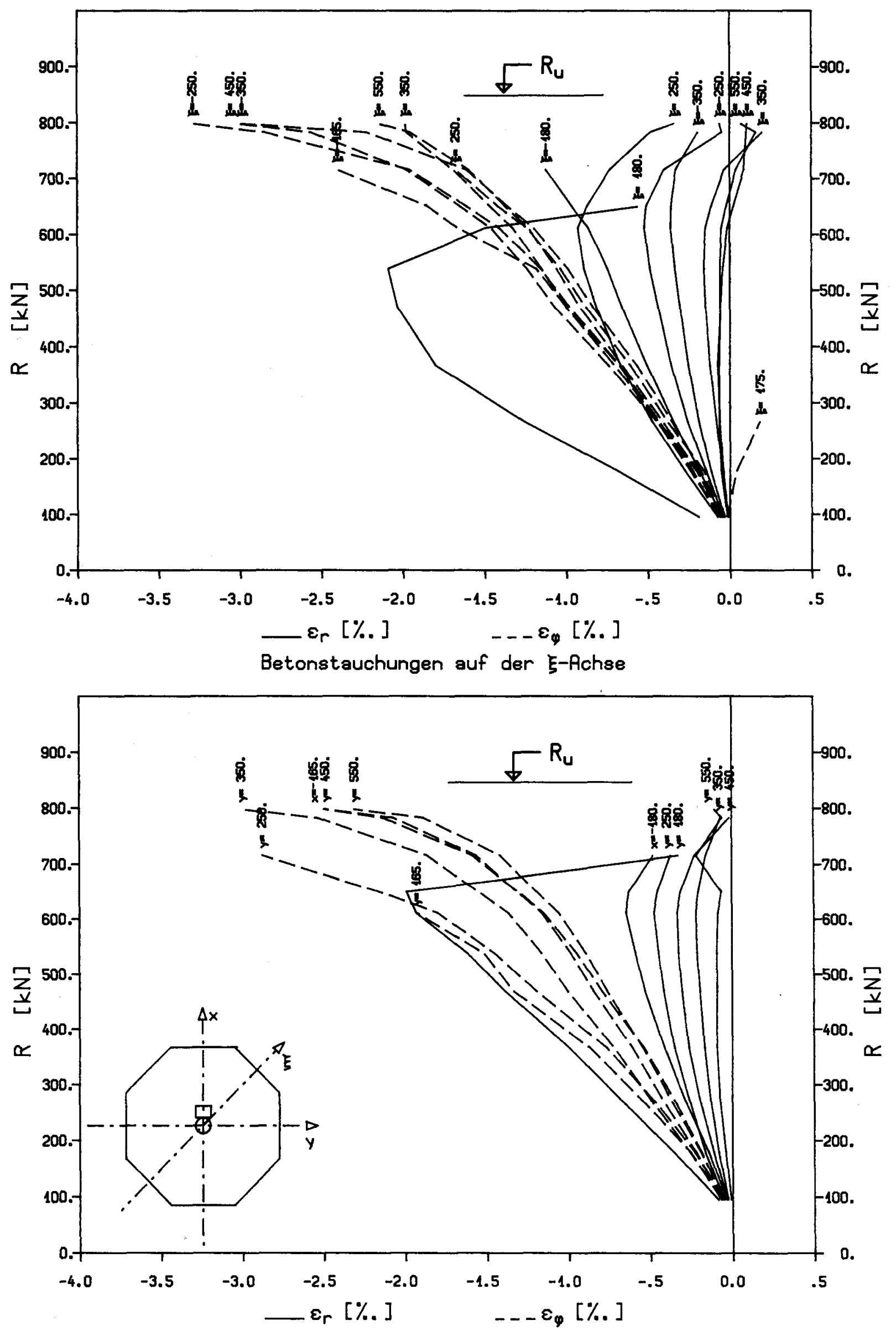

Betonstauchungen auf der $y$-Achse und auf der $x$-Achse

Bild 61: Betonstauchungen der Plattenunterselte, messstellenwelse, Platte P 17 

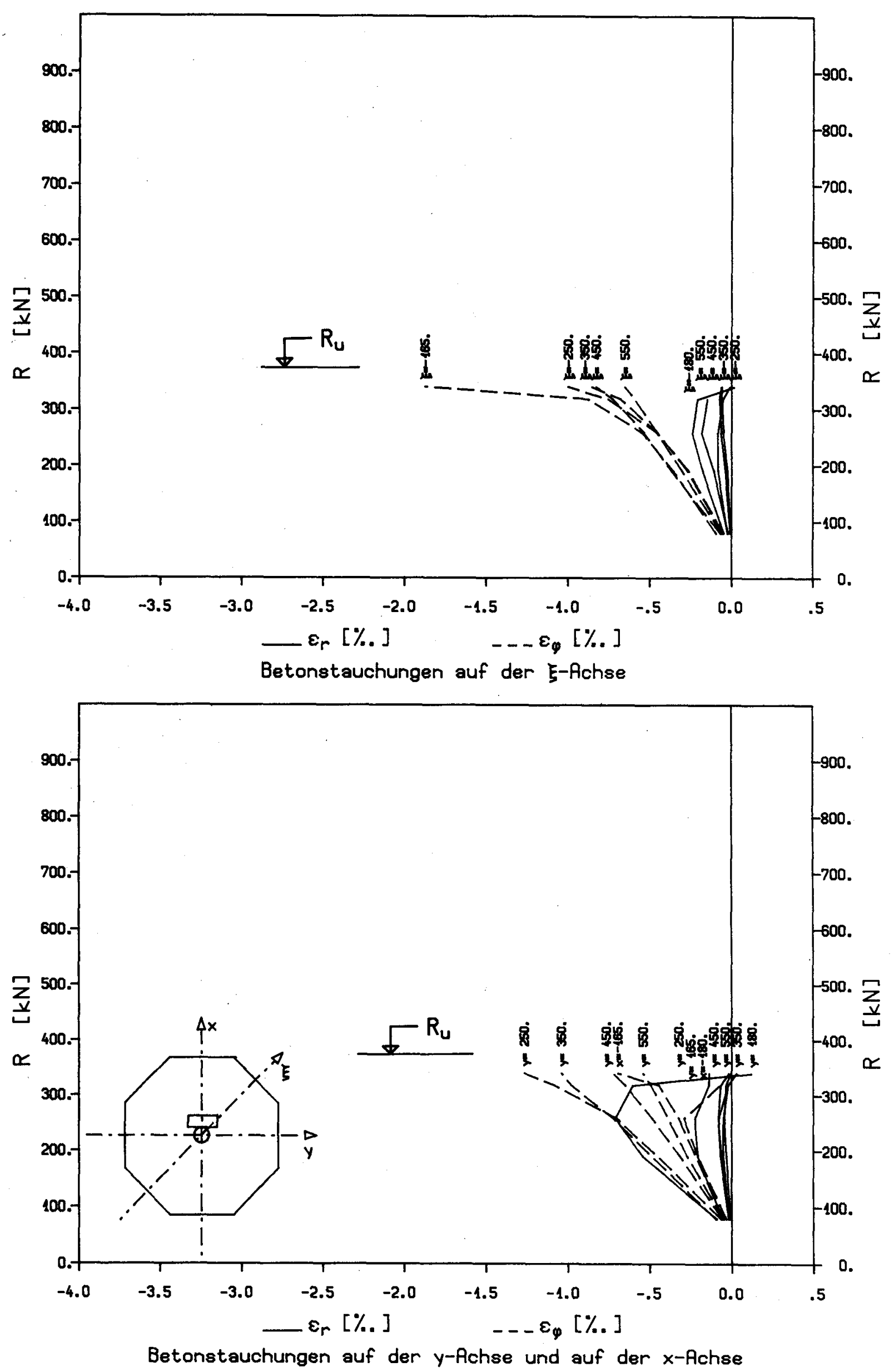

B11d 62: Betonstauchungen der Plattenunterseite, messstellenweise, Platte P 18 

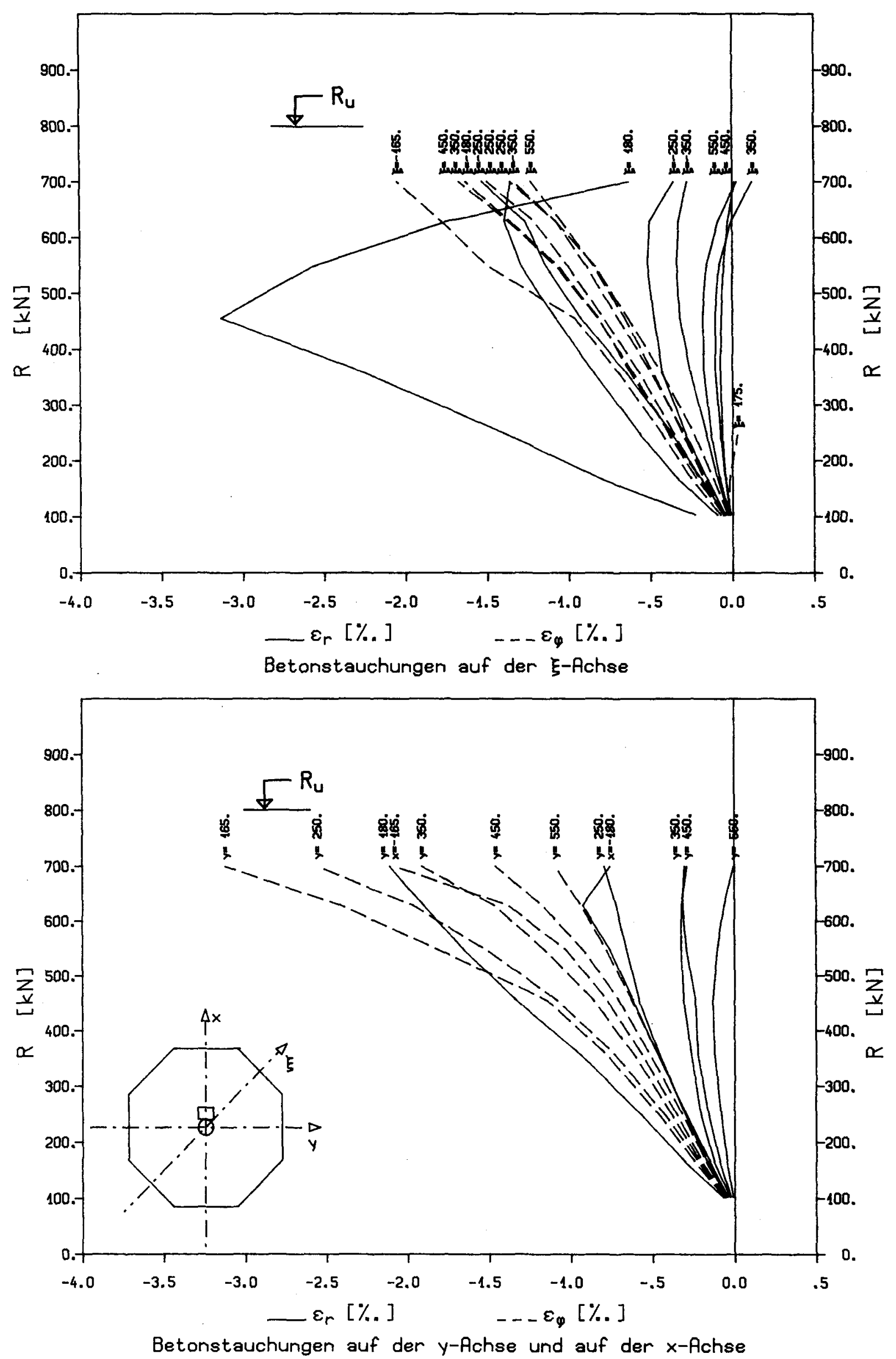

Blld 63: Betonstauchungen der Plattenunterselte, messtellenwelse, Platte $P 19$ 

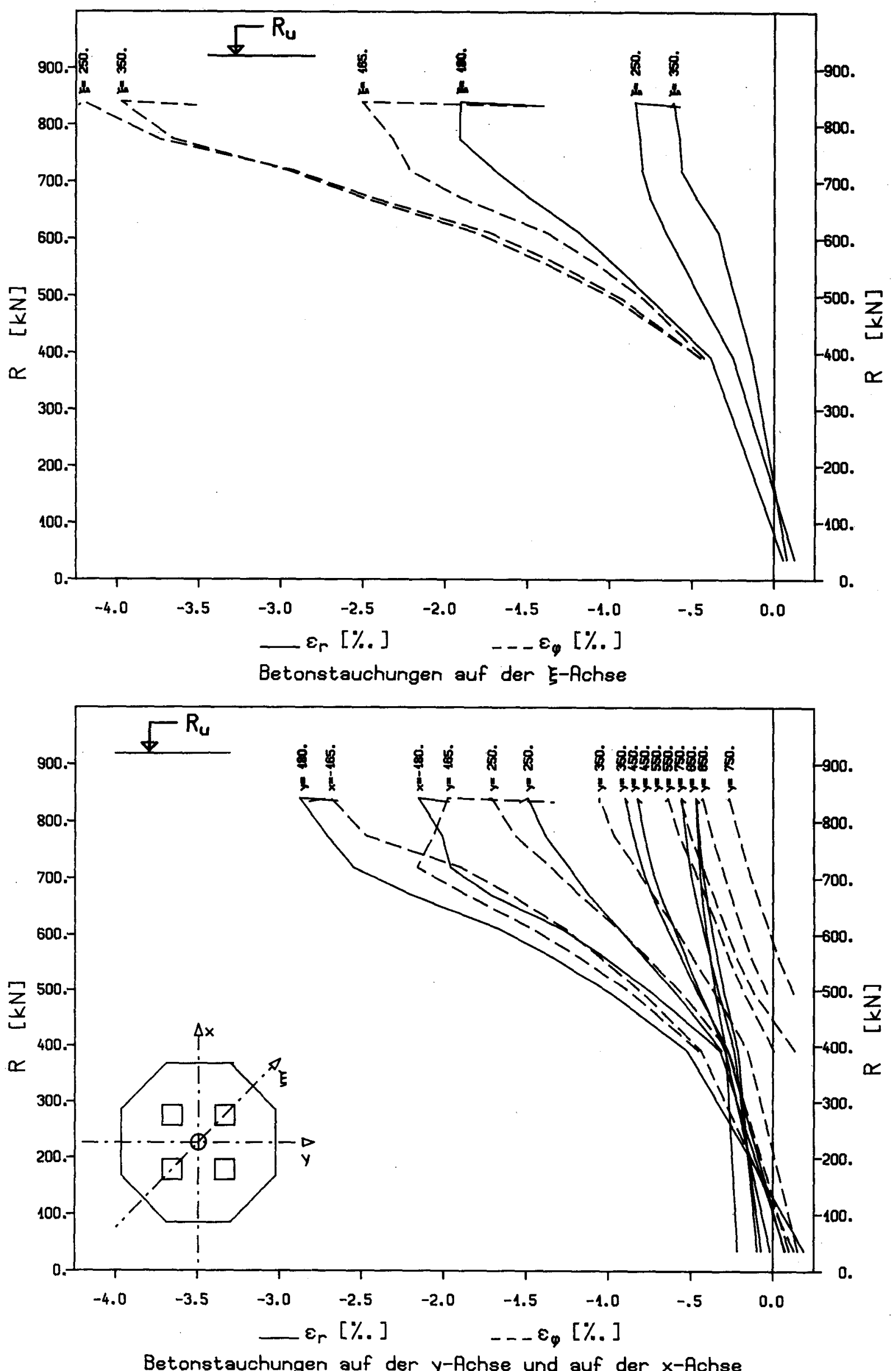

B1ld 64: Betonstauchungen der Plattenunterseite, messstellenwelse, Platte P 20 


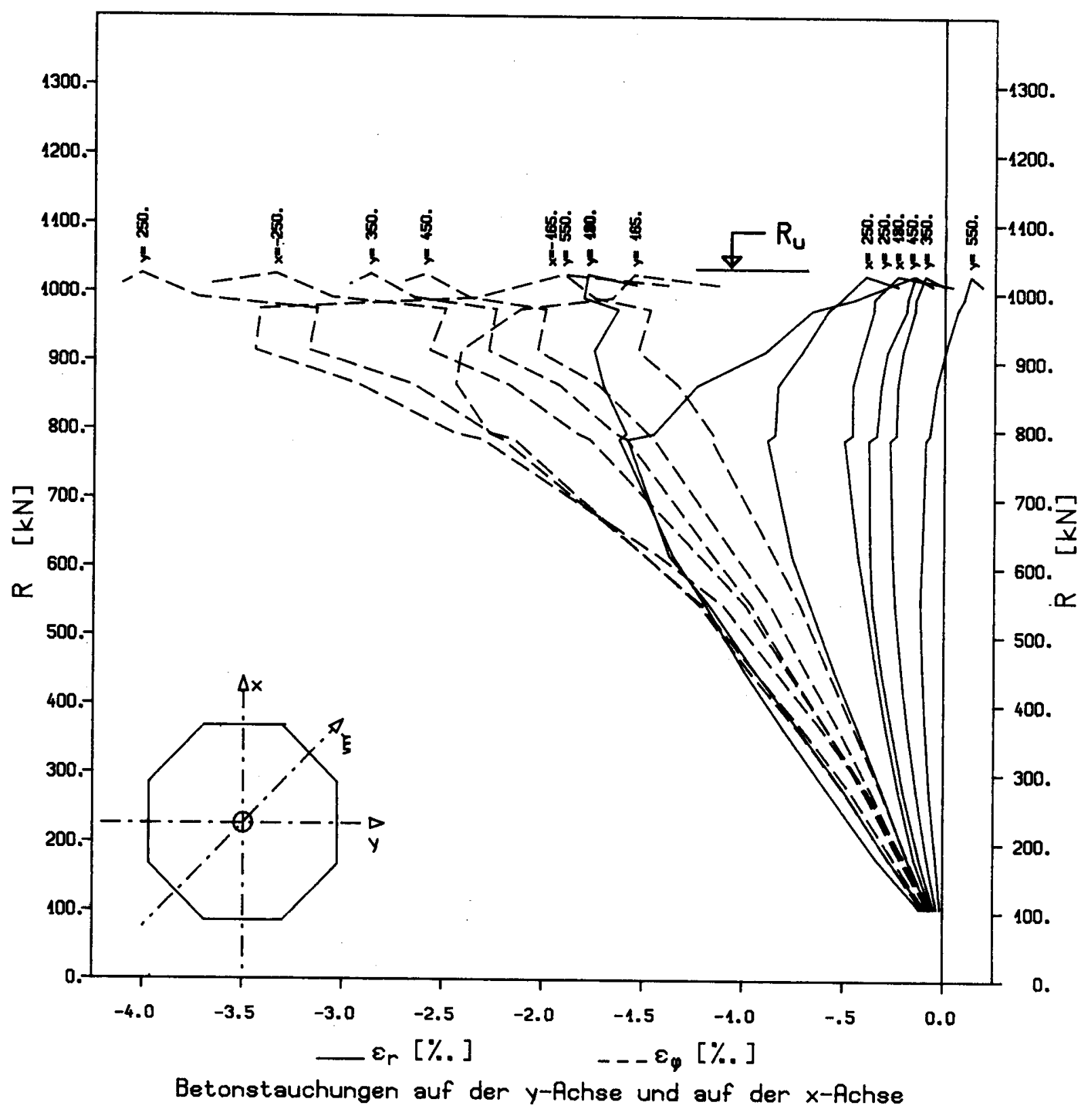

Bild 65: Betonstauchungen der Plattenunterseite, messstellenweise, Platte $P 22$ 
$\varphi \quad[\operatorname{grad}]$

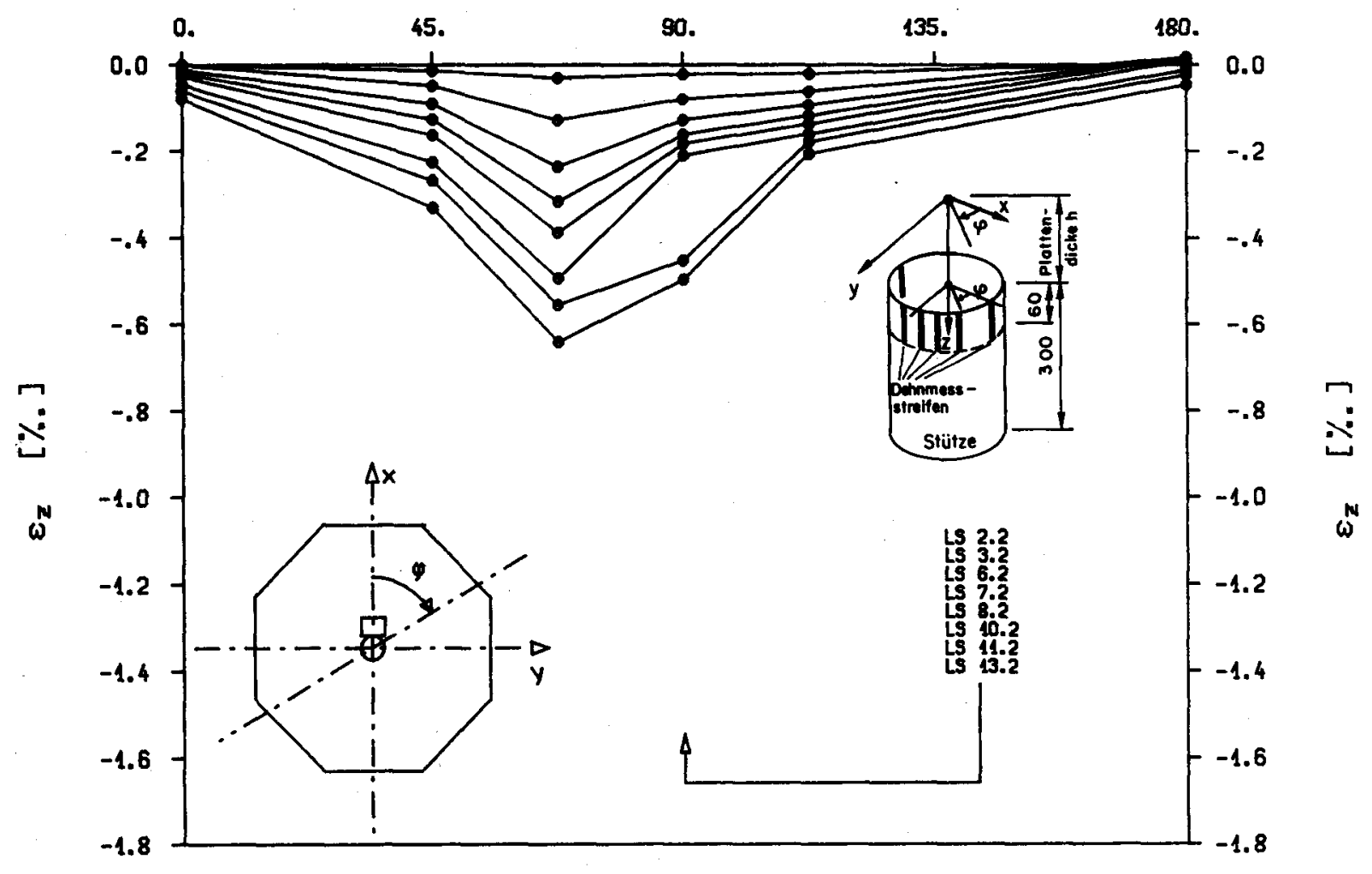

Bild 66: Axiale Betonstauchungen der Stutze, Platte P 16

\section{$\varphi \quad[g r a d]$}

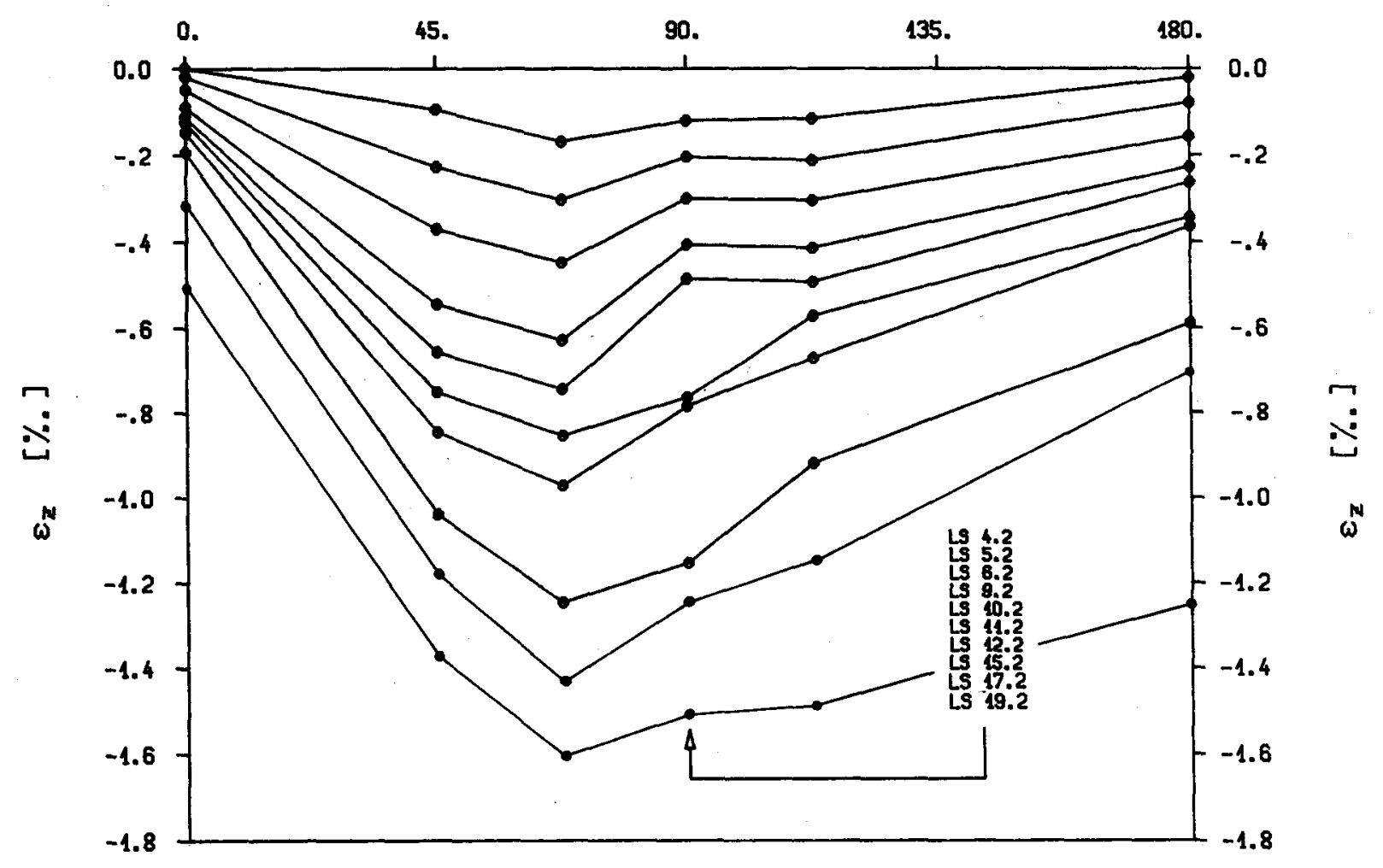

Bild 67: Axlale Betonstauchungen der Stutze, Platte P 17 


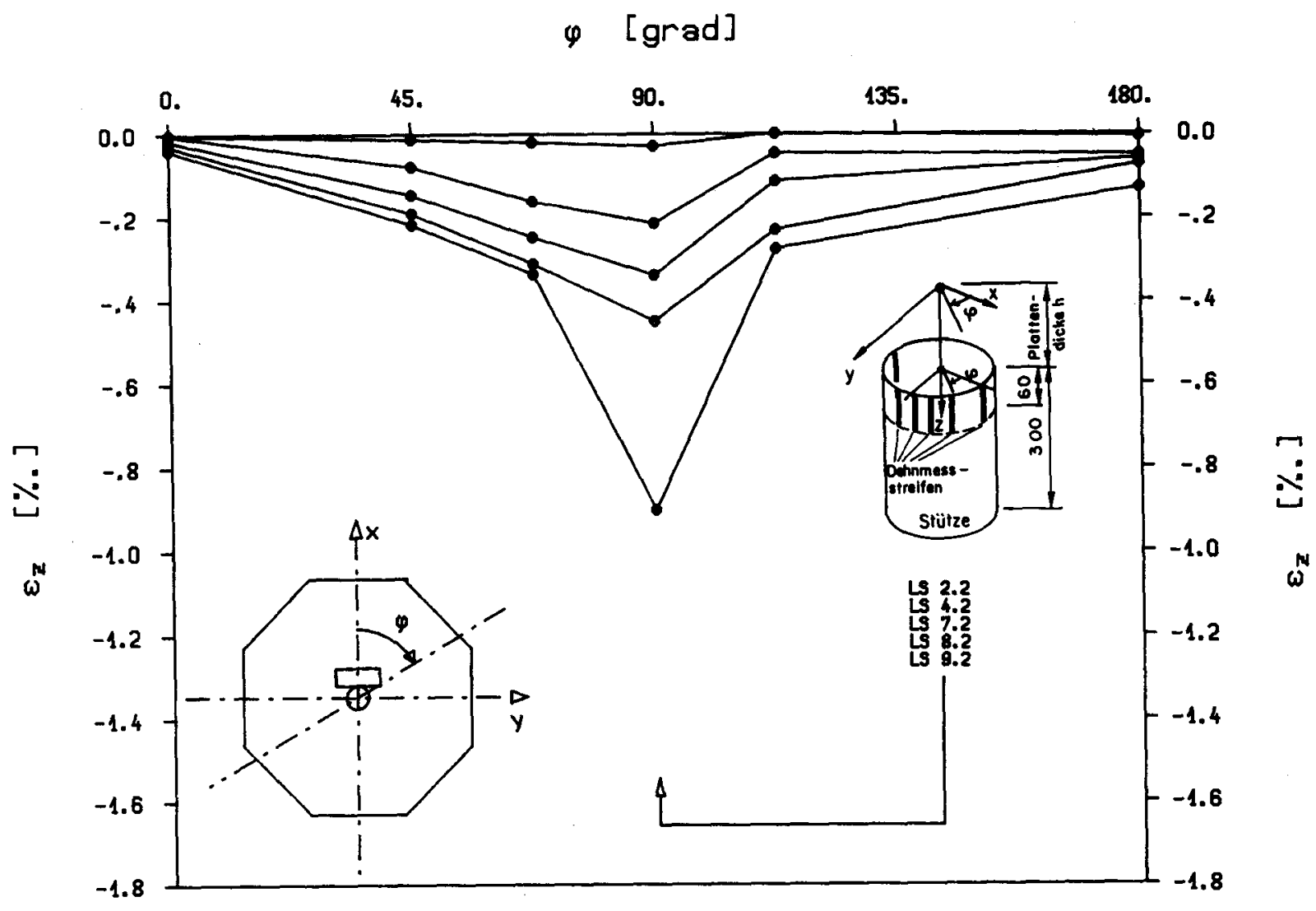

Bild 68: Axlale Betonstauchungen der Stultze, Platte P 18

\section{$\varphi \quad$ [grad]}

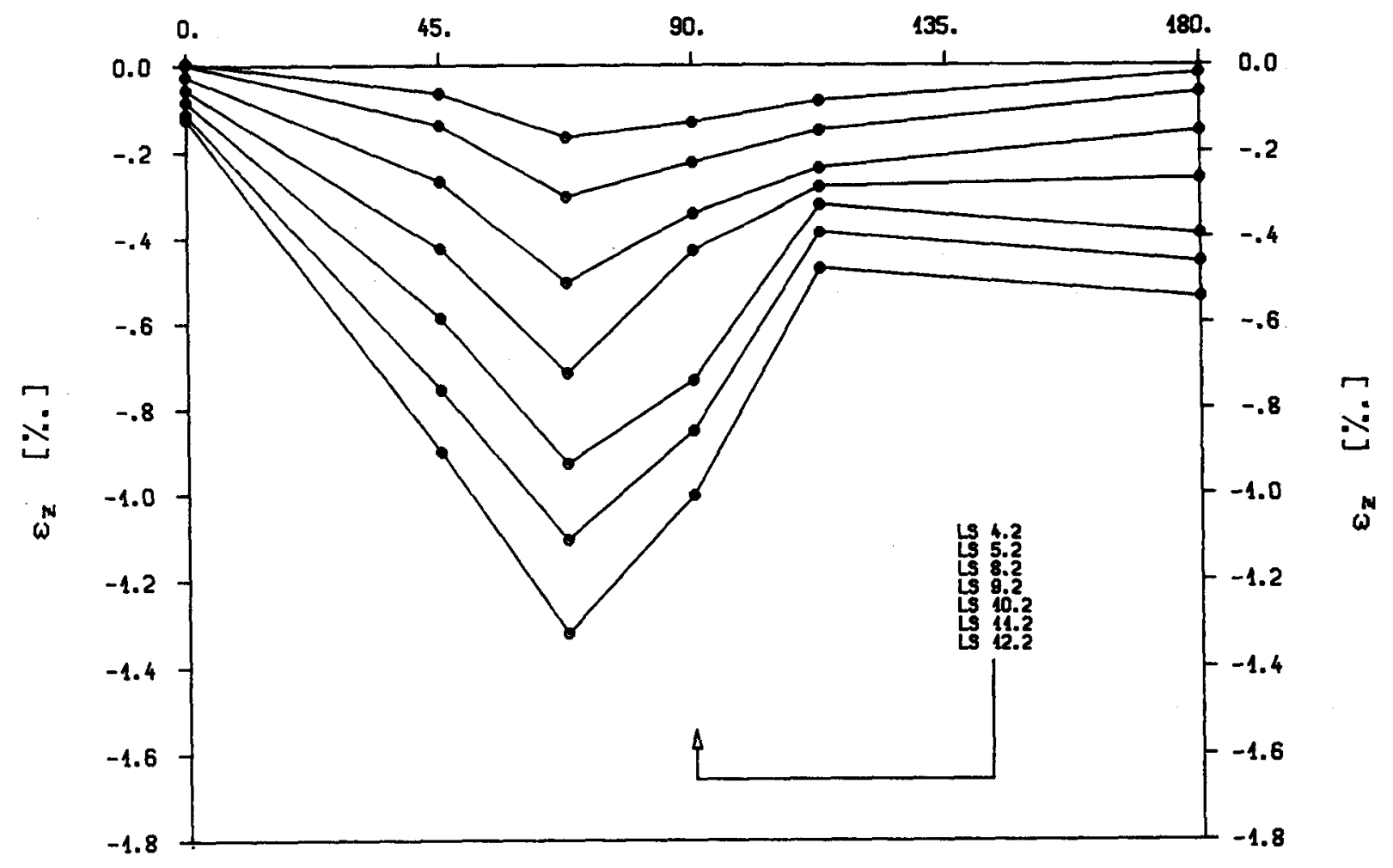

Bild 69: Axiale Betonstauchungen der Stutze, Platte P 19 


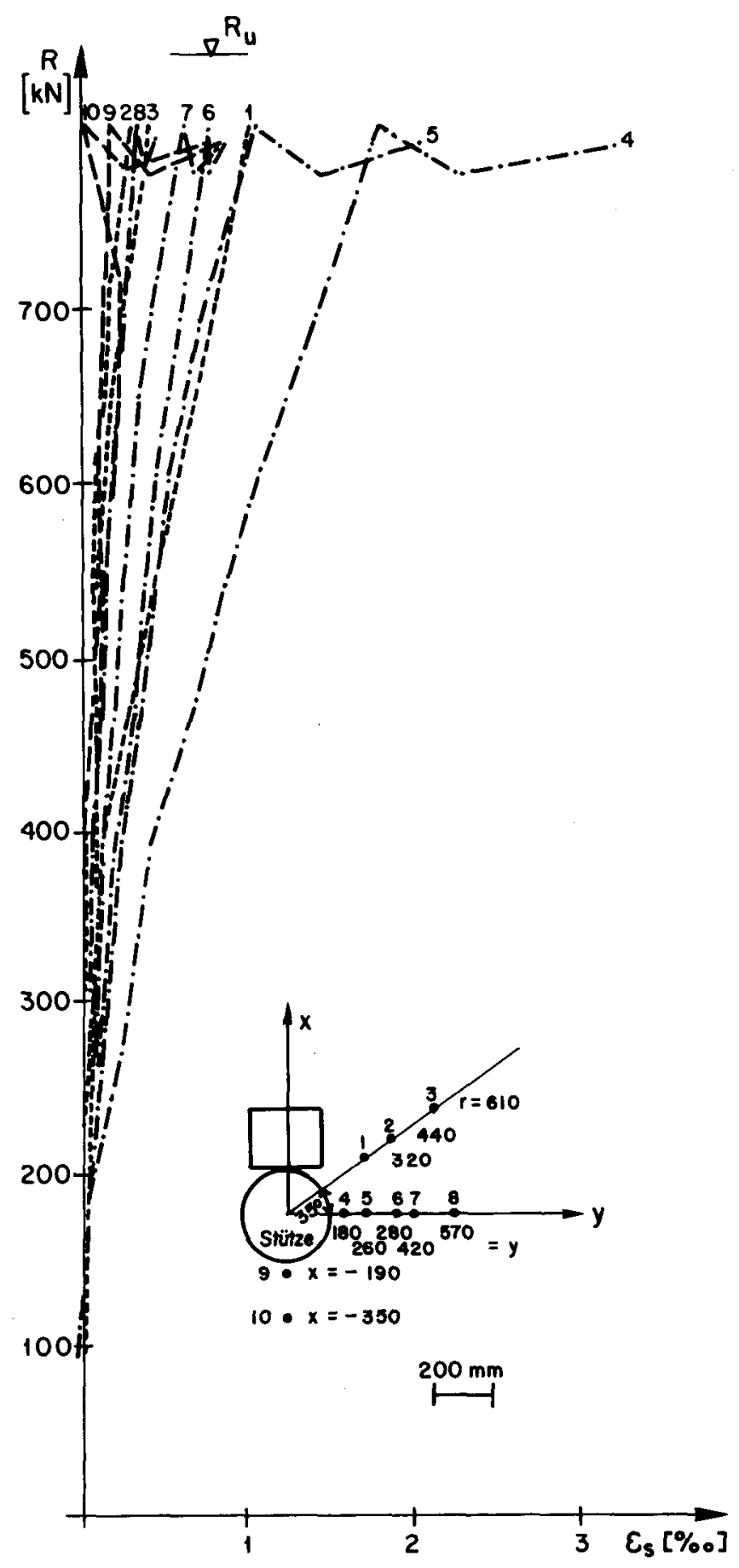

a) Lost $R$ als Funktion der Bügeldehnung $\varepsilon_{s}$

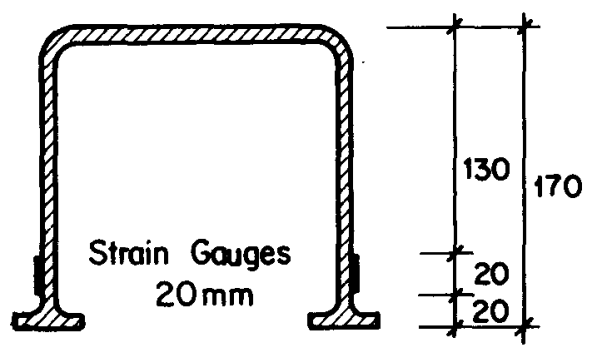

c) Lage der Dehnmessstreifen (Strain Gouges)
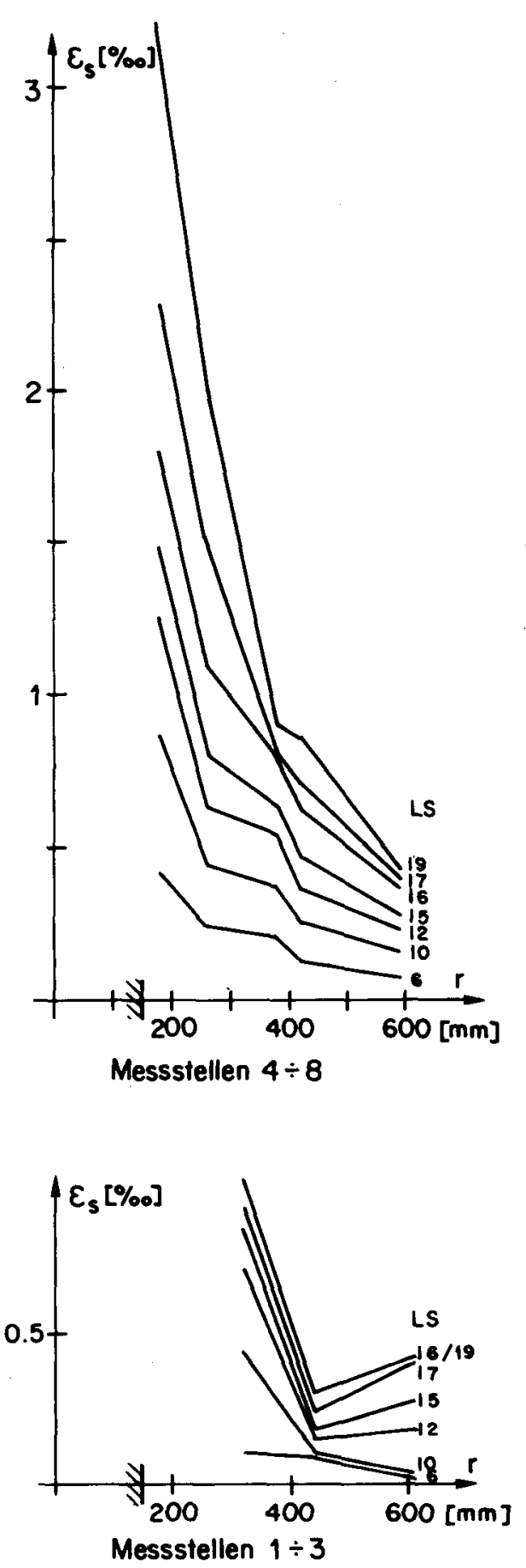

b) Radialer Verlauf der Bügeldehnungen

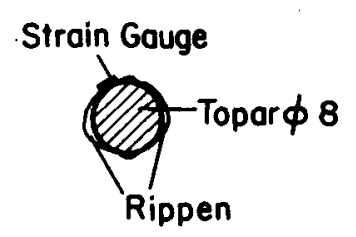




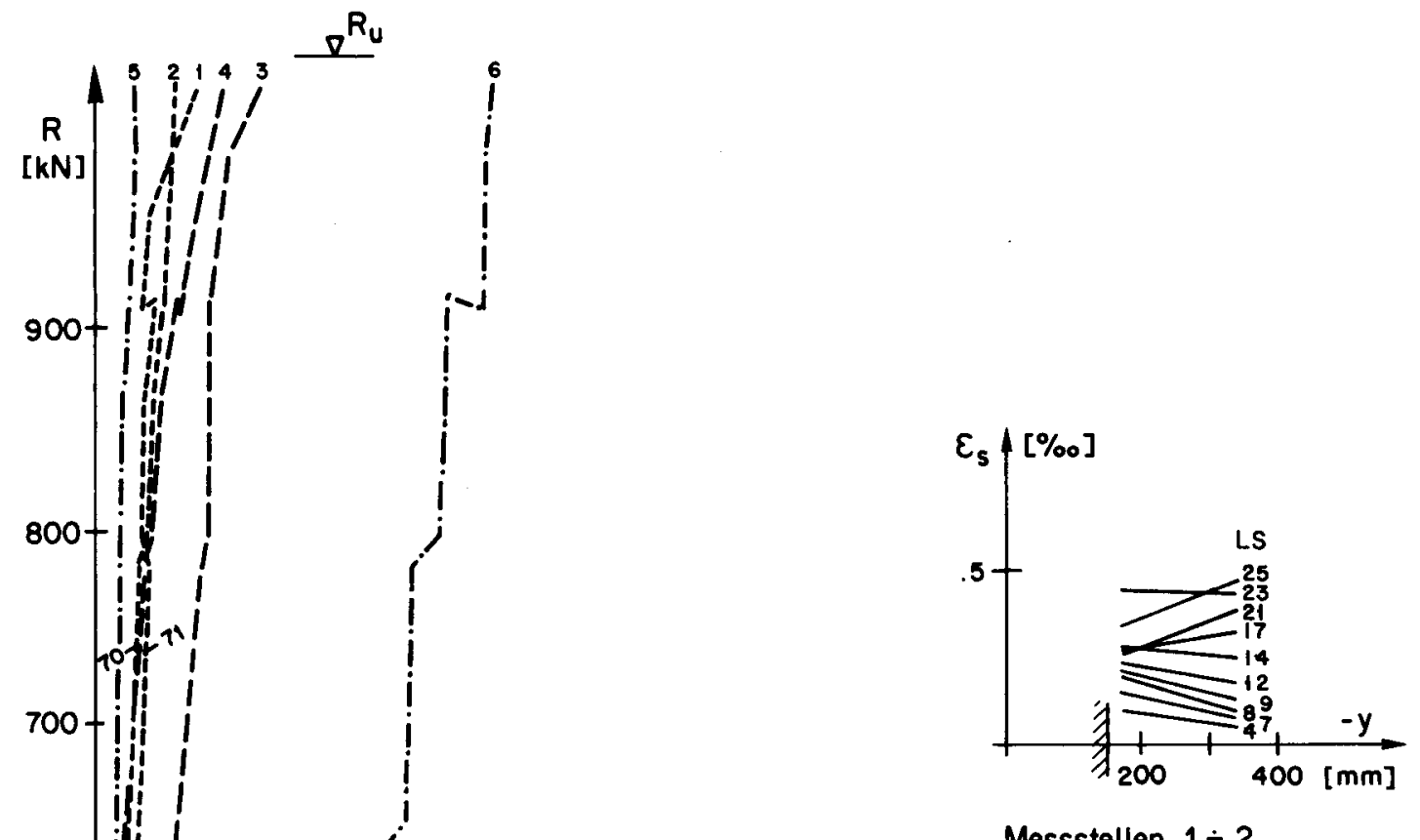

Messstellen $1 \div 2$

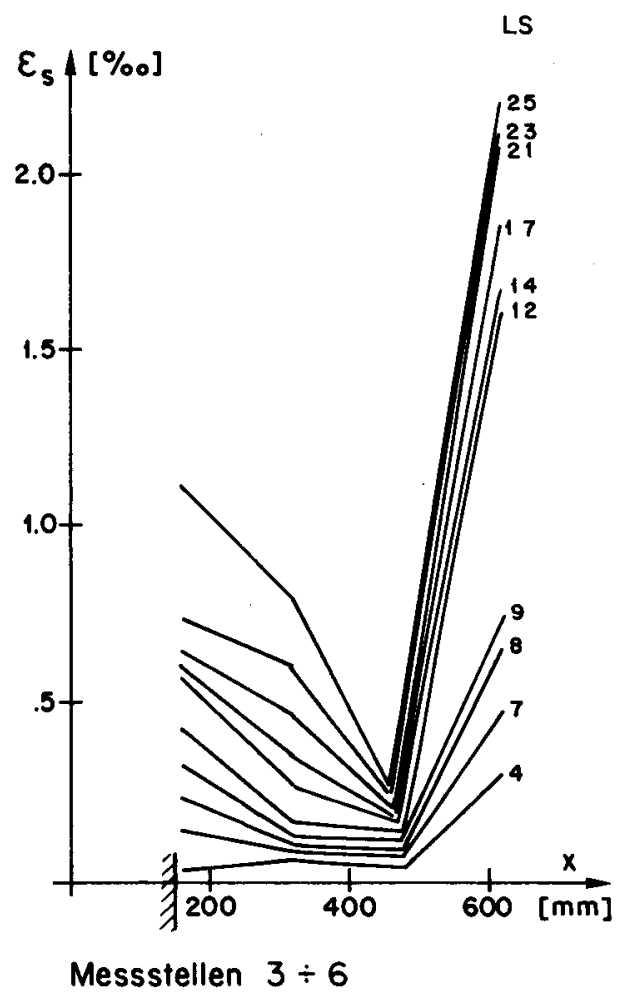

o) Last $R$ als Funktion der Bügeldehnung $\varepsilon_{s}$

b) Radialer Verlauf der Bügeldehnnungen

Bild 71: Stahldehnungen der Bügel, Platte $P 22$ 


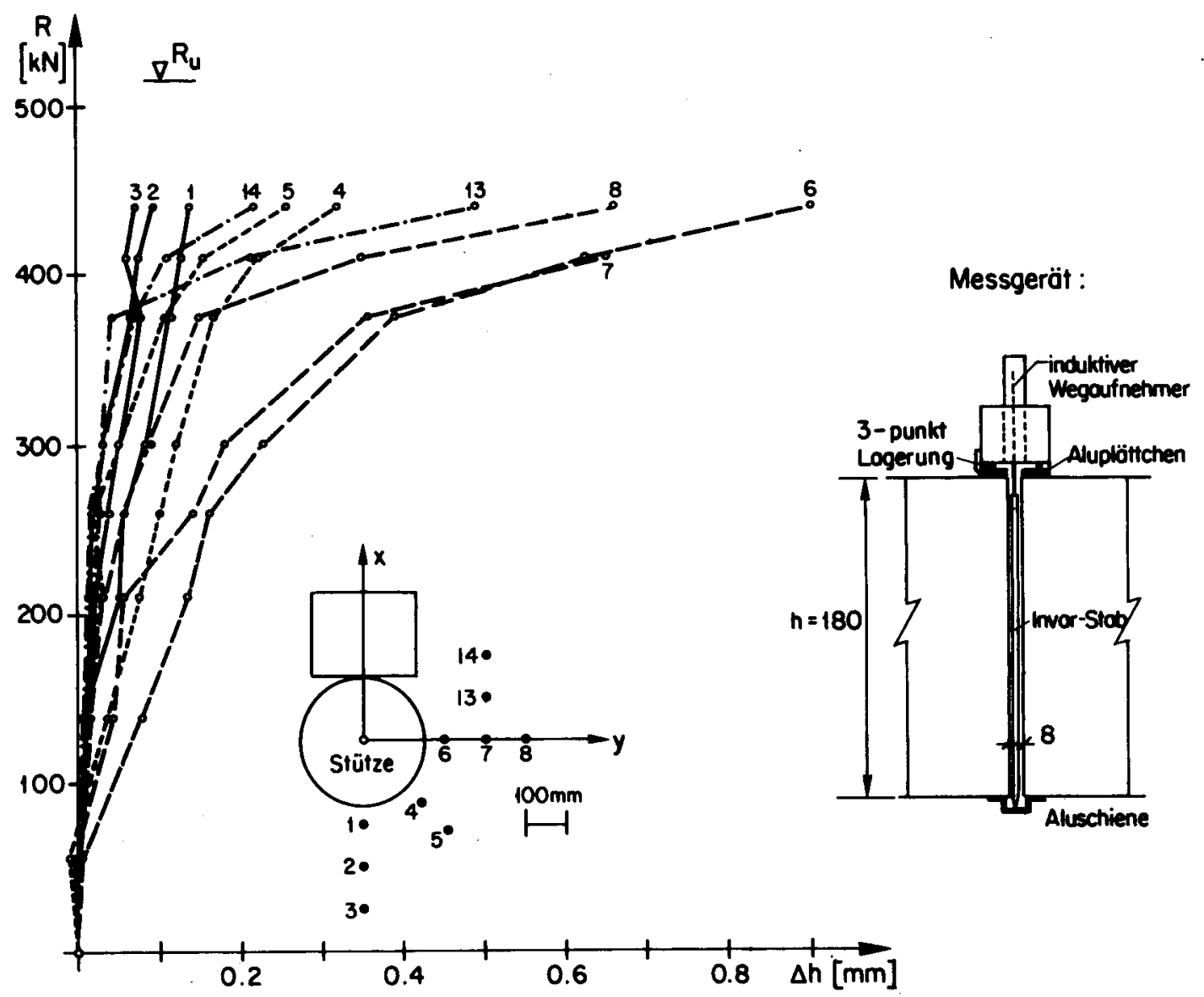

a) Lost $R$ als Funktion der Zunahme der Dicke $\Delta h$
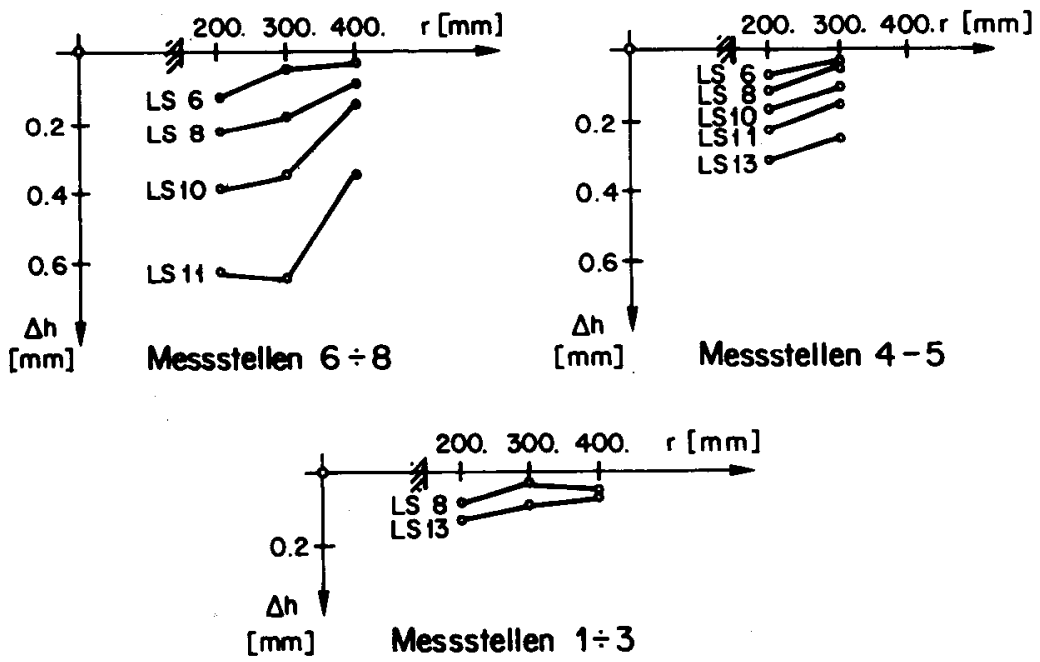

b) Radialer Verlauf von $\Delta h$

Bild 72: Zunahme der Plattendicke, Platte P 16 


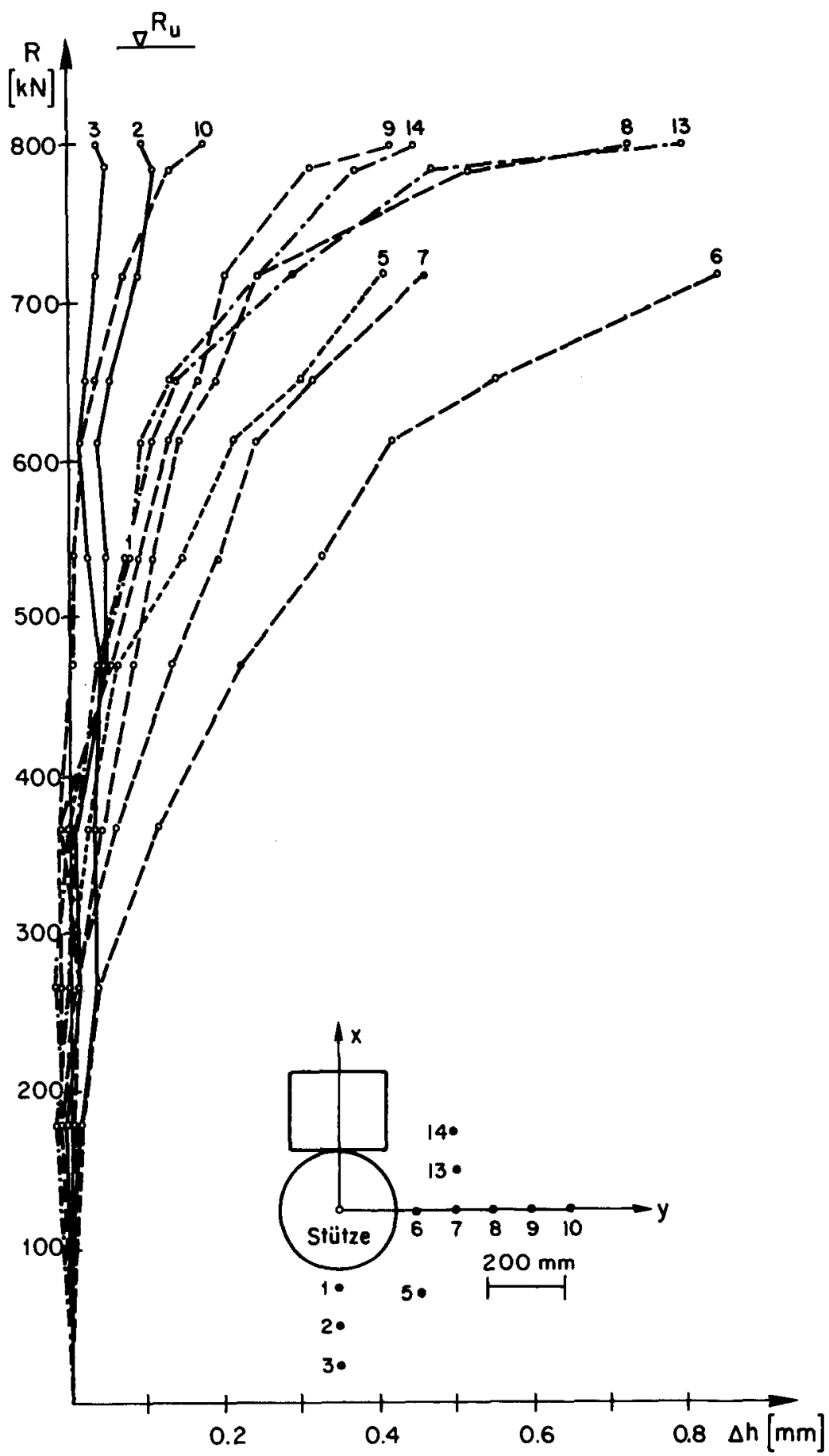

a) Lost $R$ als Funktion der Zunahme der Dicke $\Delta h$

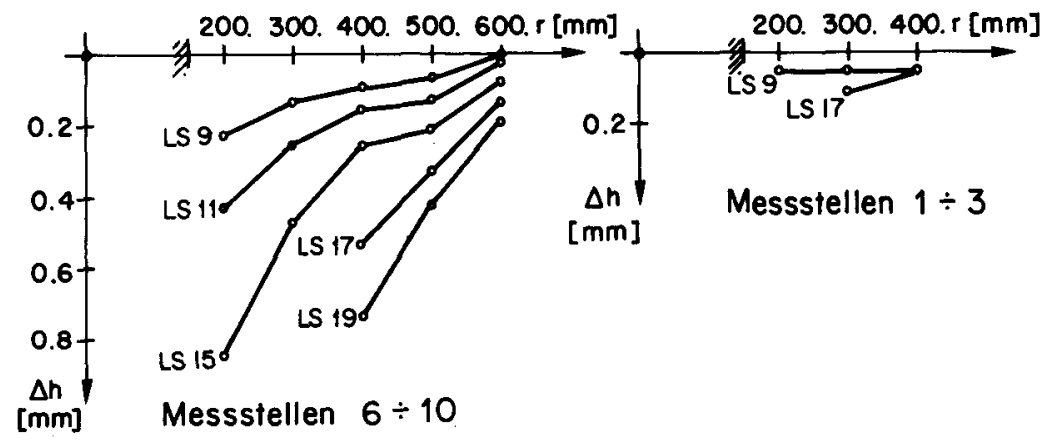

b) Zunahme der Plattendicke $\Delta \mathrm{h}$, Platte P17

Bild 73: Zunahme der Plattendicke, Platte P 17 


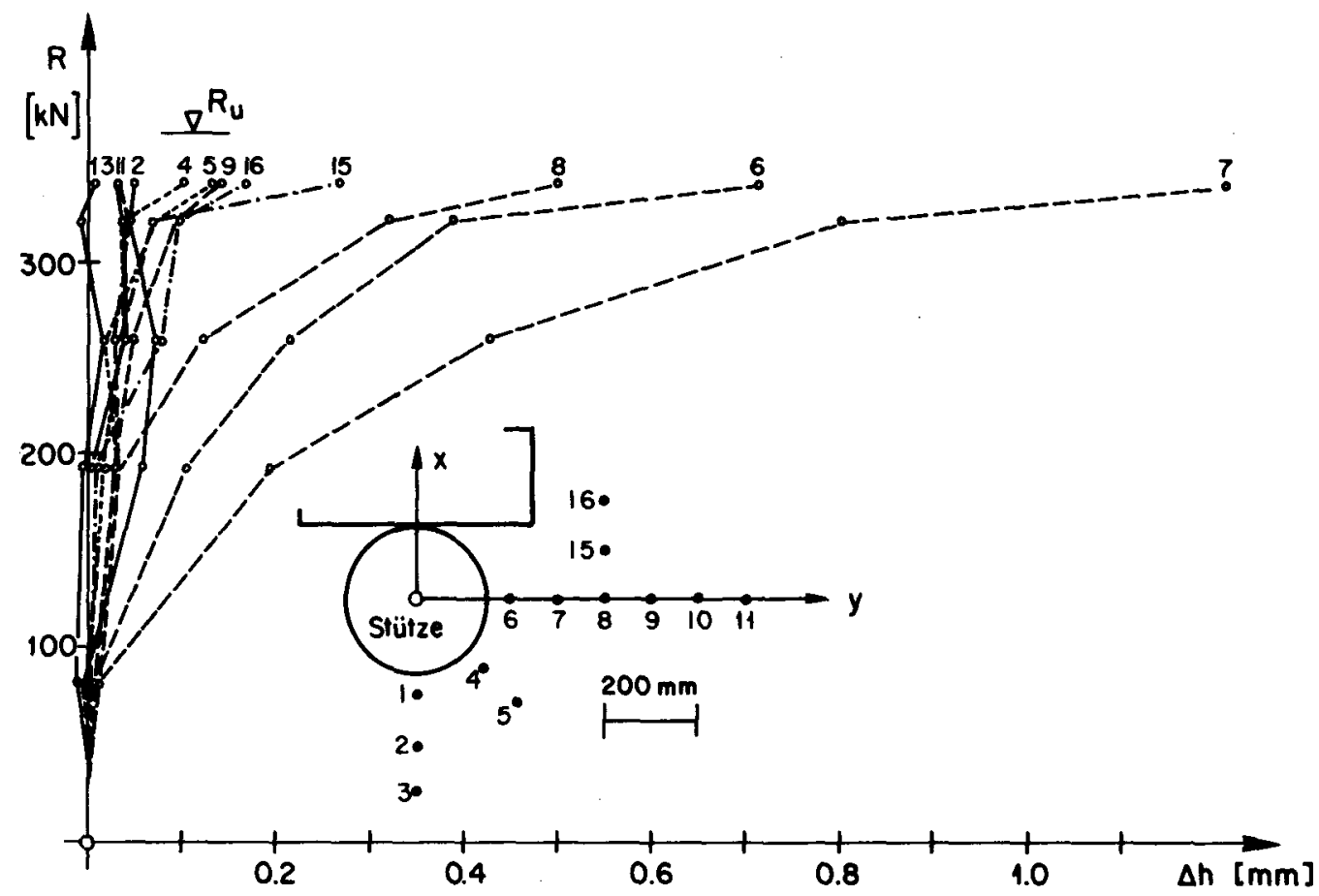

a) Last als Funktion der Zunahme der Dicke $\Delta h$
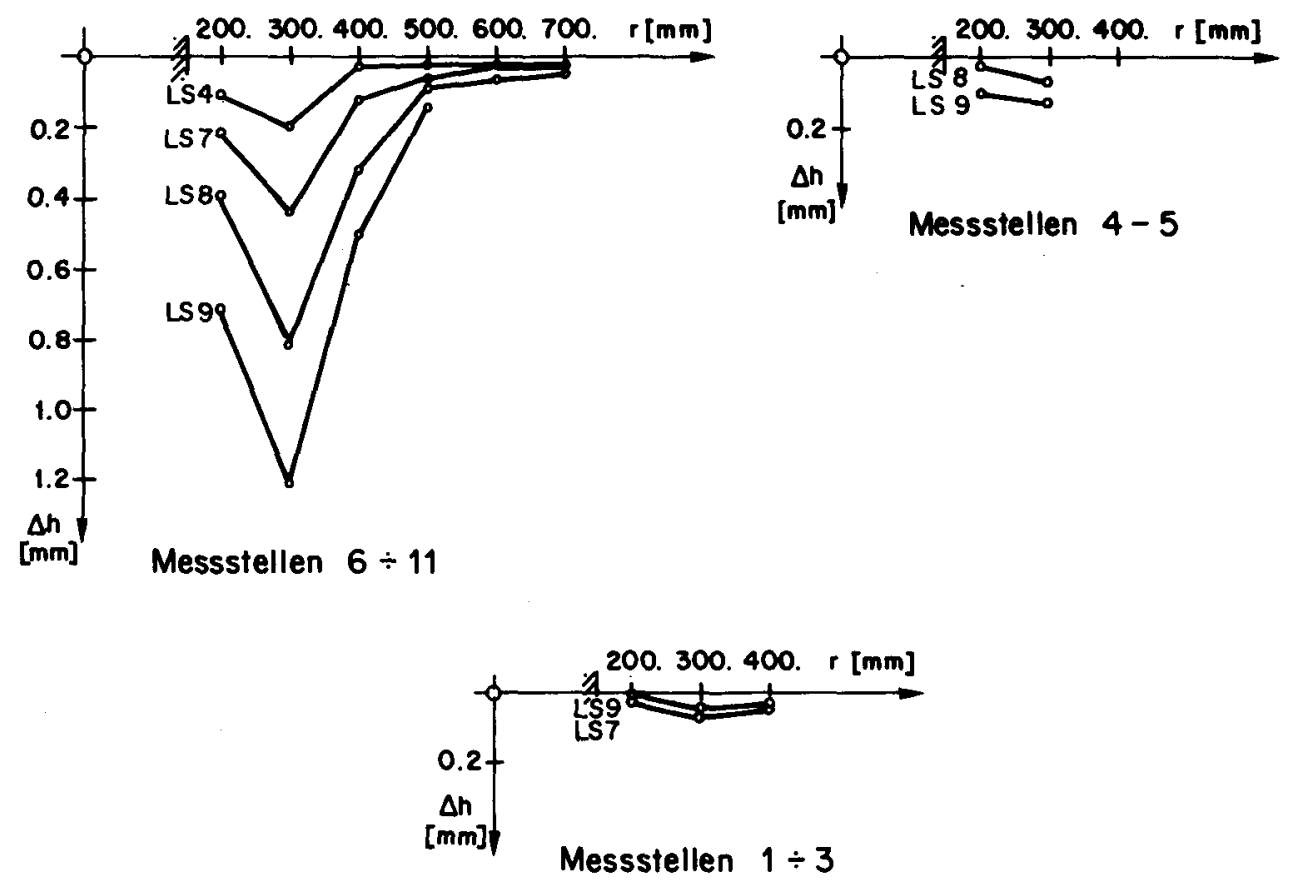

b) Rodialer Verlouf von $\Delta h$

Bild 74: Zunahme der Plattendicke, Platte P 18 


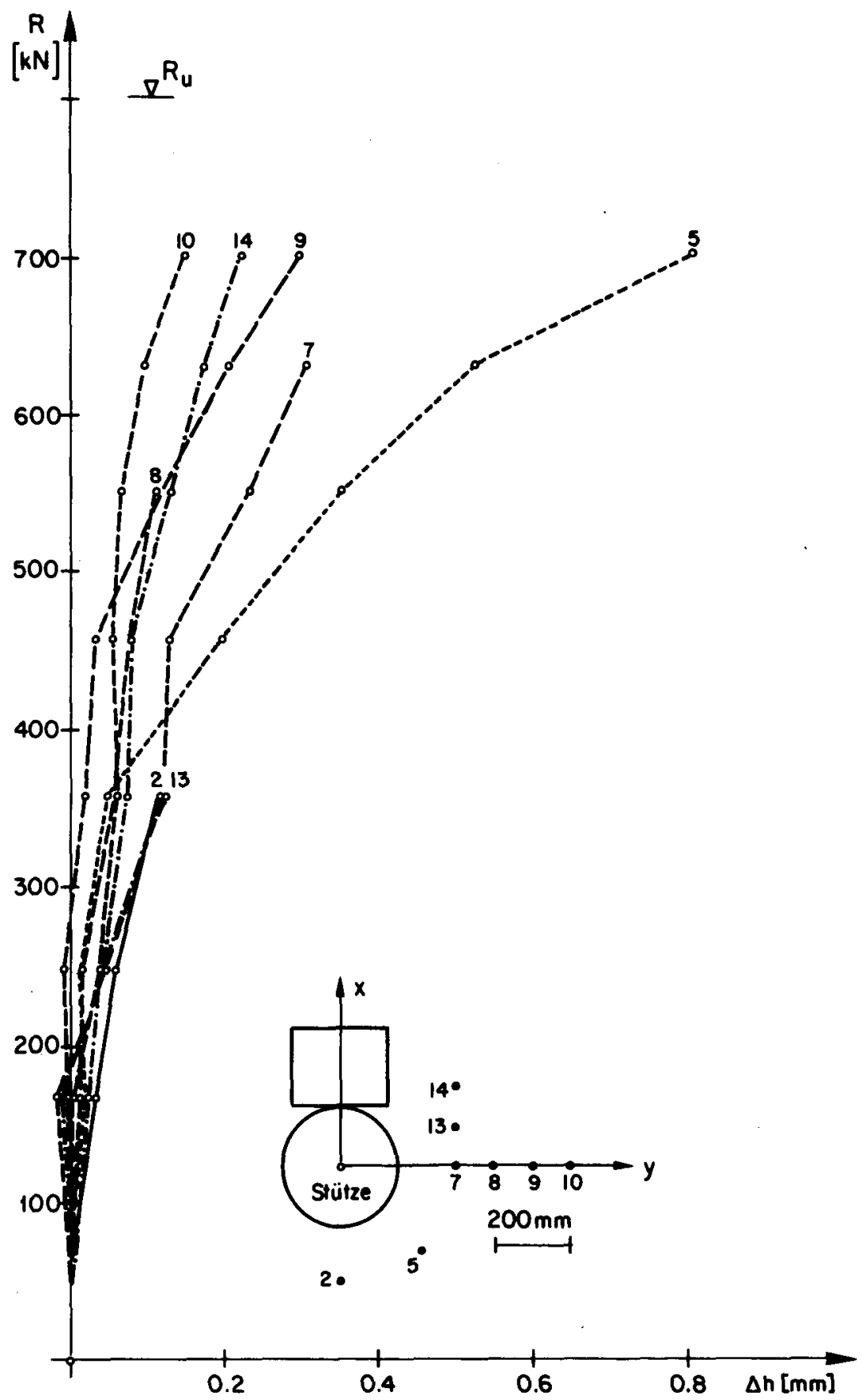

a) Lost $R$ als Funktion der Zunahme der Dicke $\Delta h$

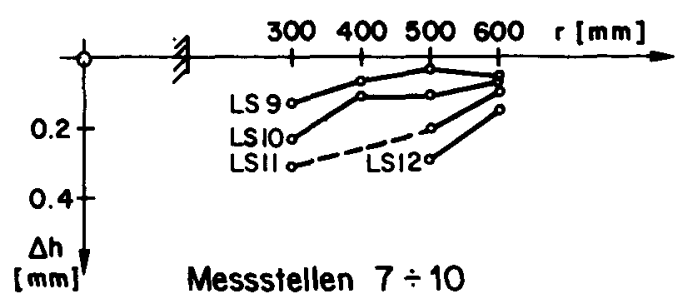

b) Rodialer Verlauf von $\Delta h$

Bild 75: Zunahme der Plattendicke, Platte P 19 


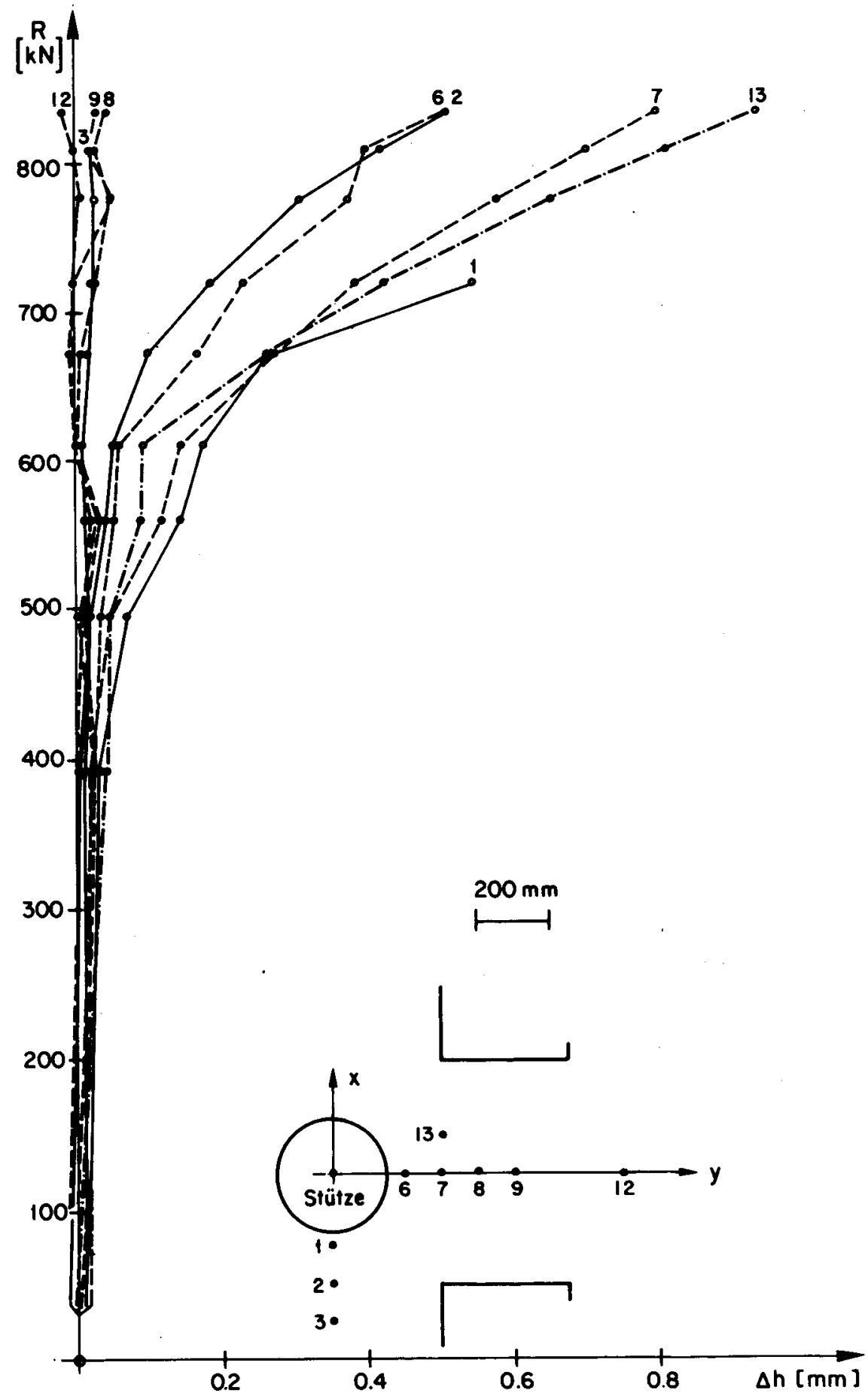

a) Last $R$ als Funktion der Zunahme der Dicke $\Delta h$

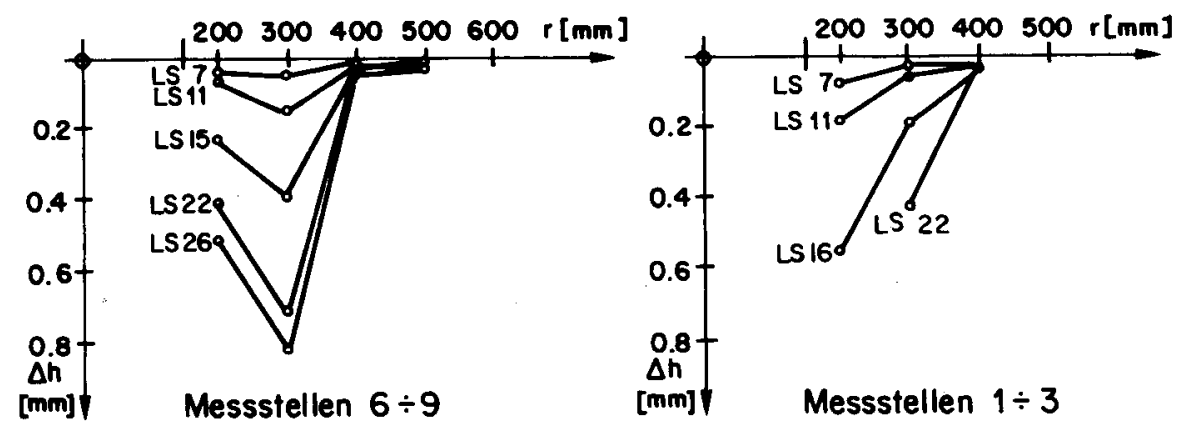

b) Rodialer Verlauf von $\Delta h$

Bild 76: Zunahme der Plattendicke, Platte P 20 


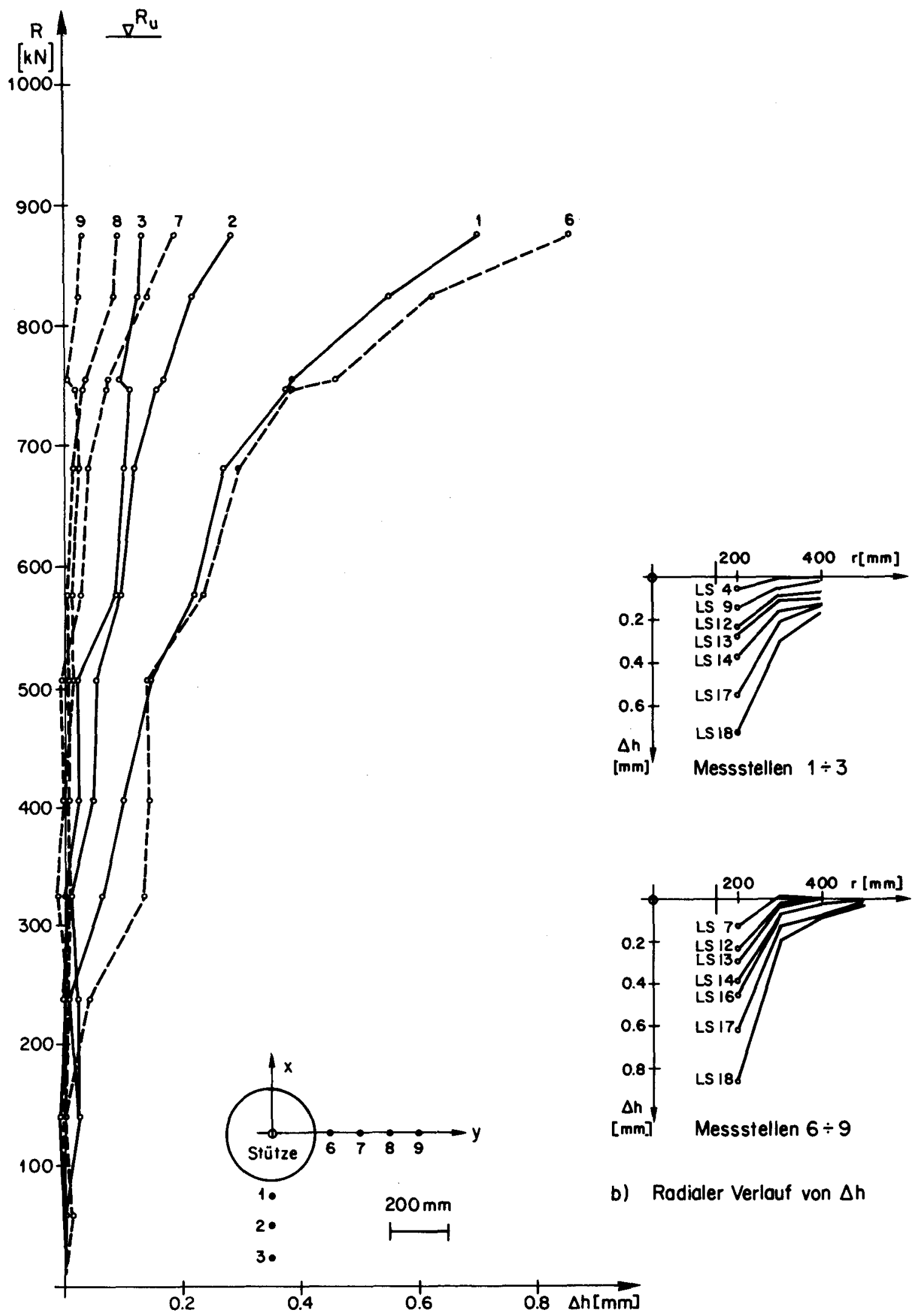

a) Lost $R$ als Funktion der Zunahme der Dicke $\Delta h$

Bild 77: Zunahme der Plattendicke, Platte P 22 

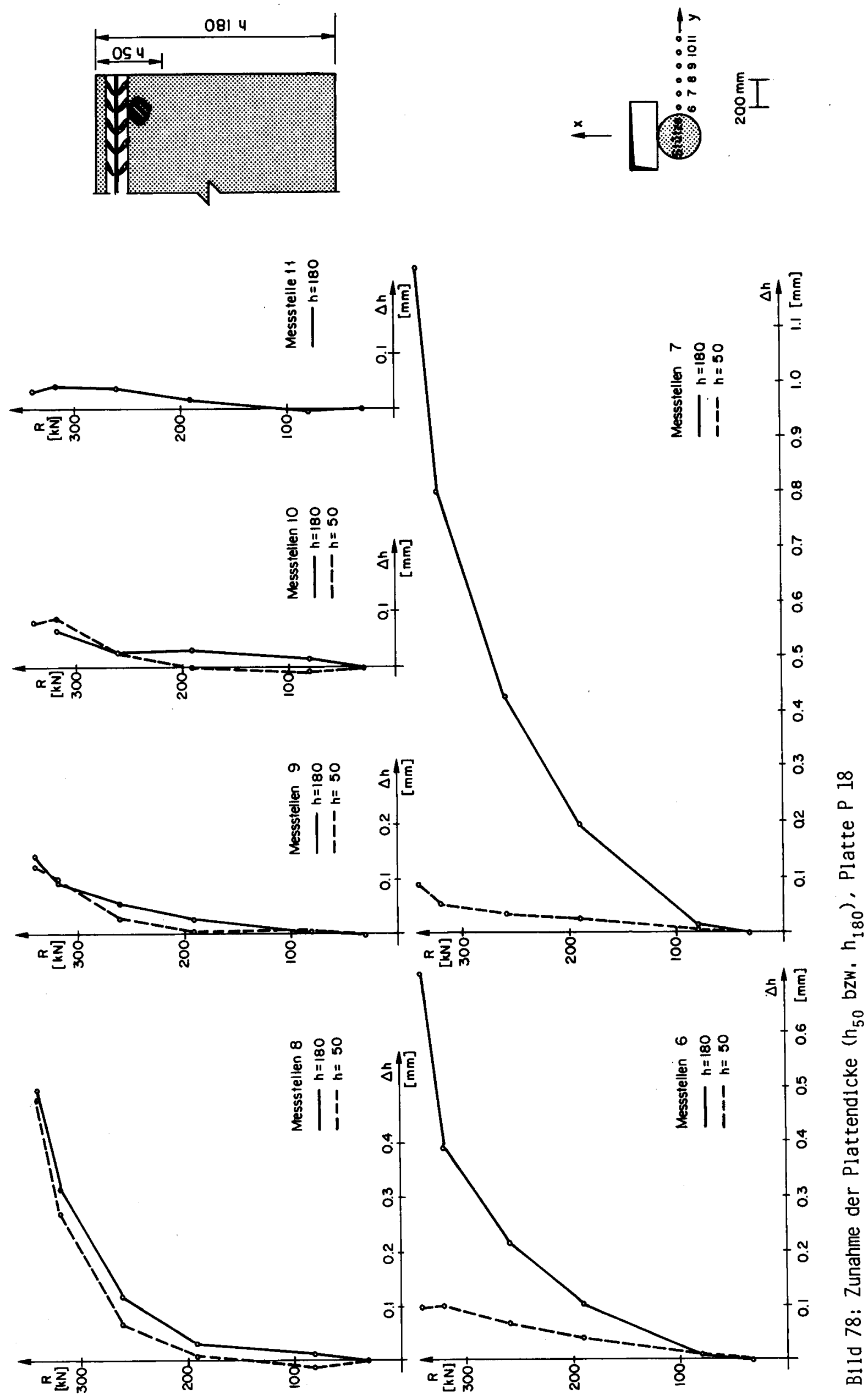

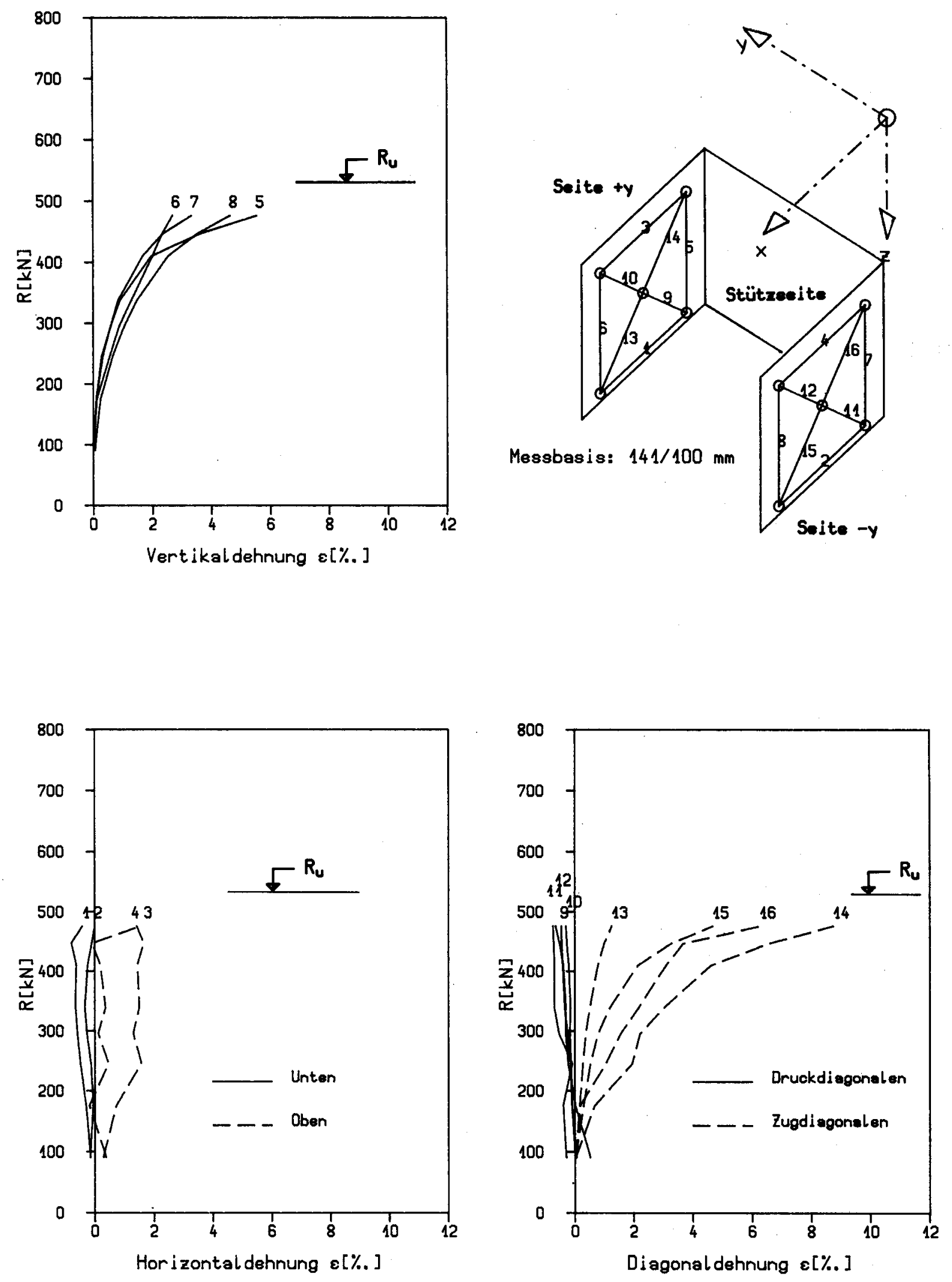

Dehnungen in Funktion der Last, messstel lenweise

B1ld 79: Mittlere Dehnungen in der Aussparung, Platte P 16 

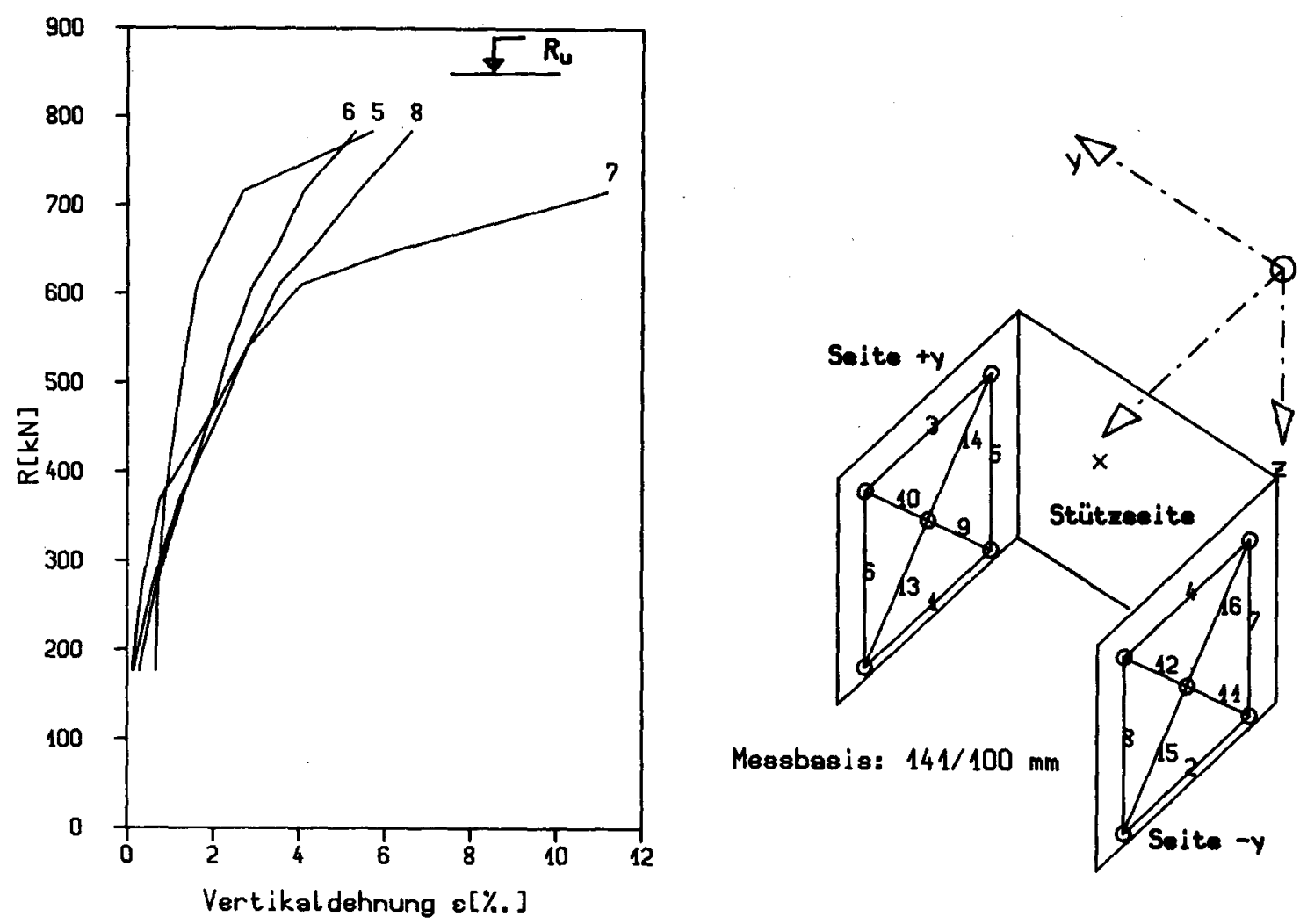

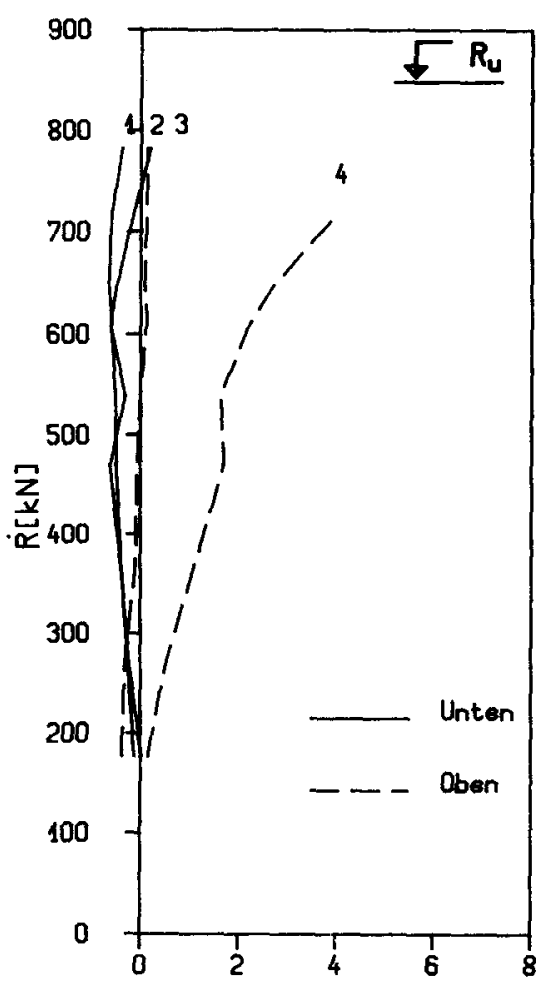

Horizontaldehnung $\varepsilon[\%]$

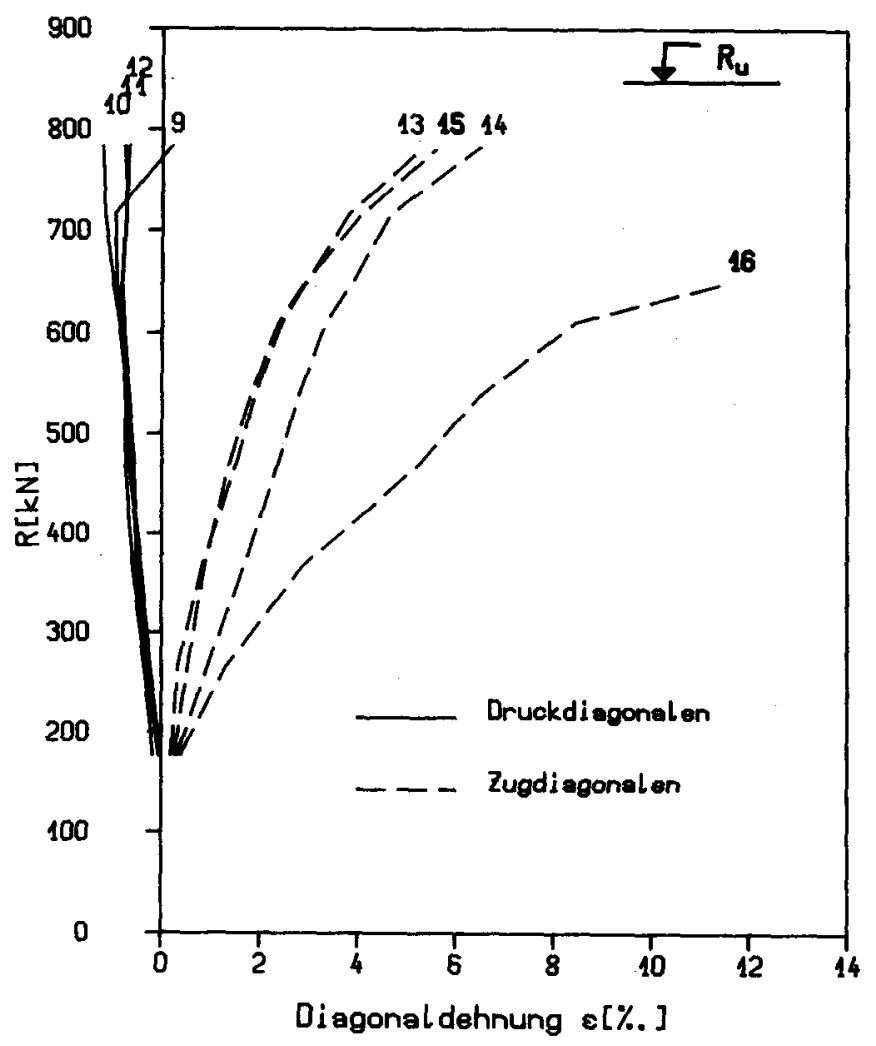

Dehnungen in Funktion der Last, messstellenwe ise

B1ld 80: Mittlere Dehnungen in der Aussparung, Platte P 17 

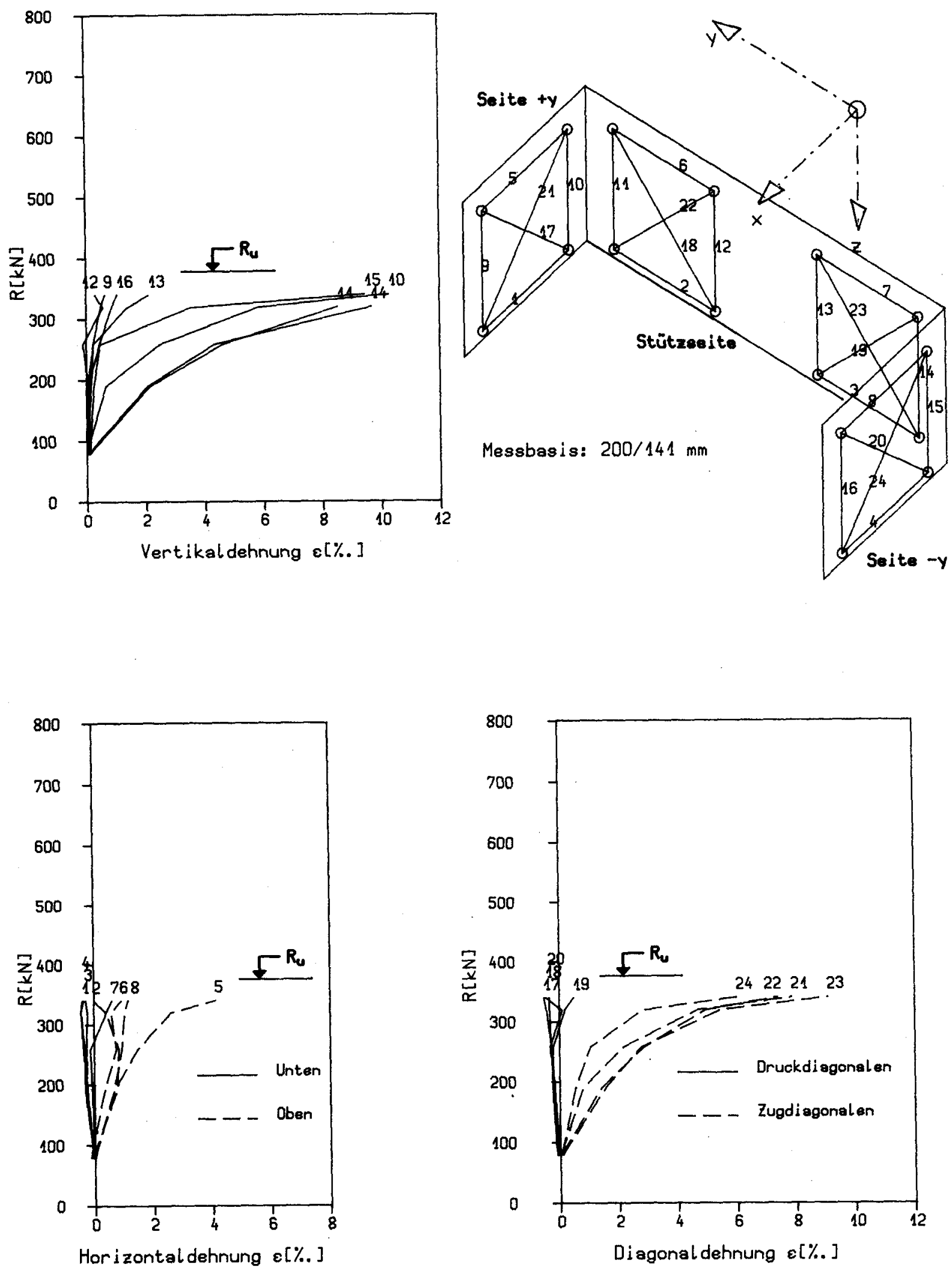

Dehnungen in Funktion der Last, messstellenweise

Bild 81: Mittlere Dehnungen in der Aussparung, Platte $P 18$ 

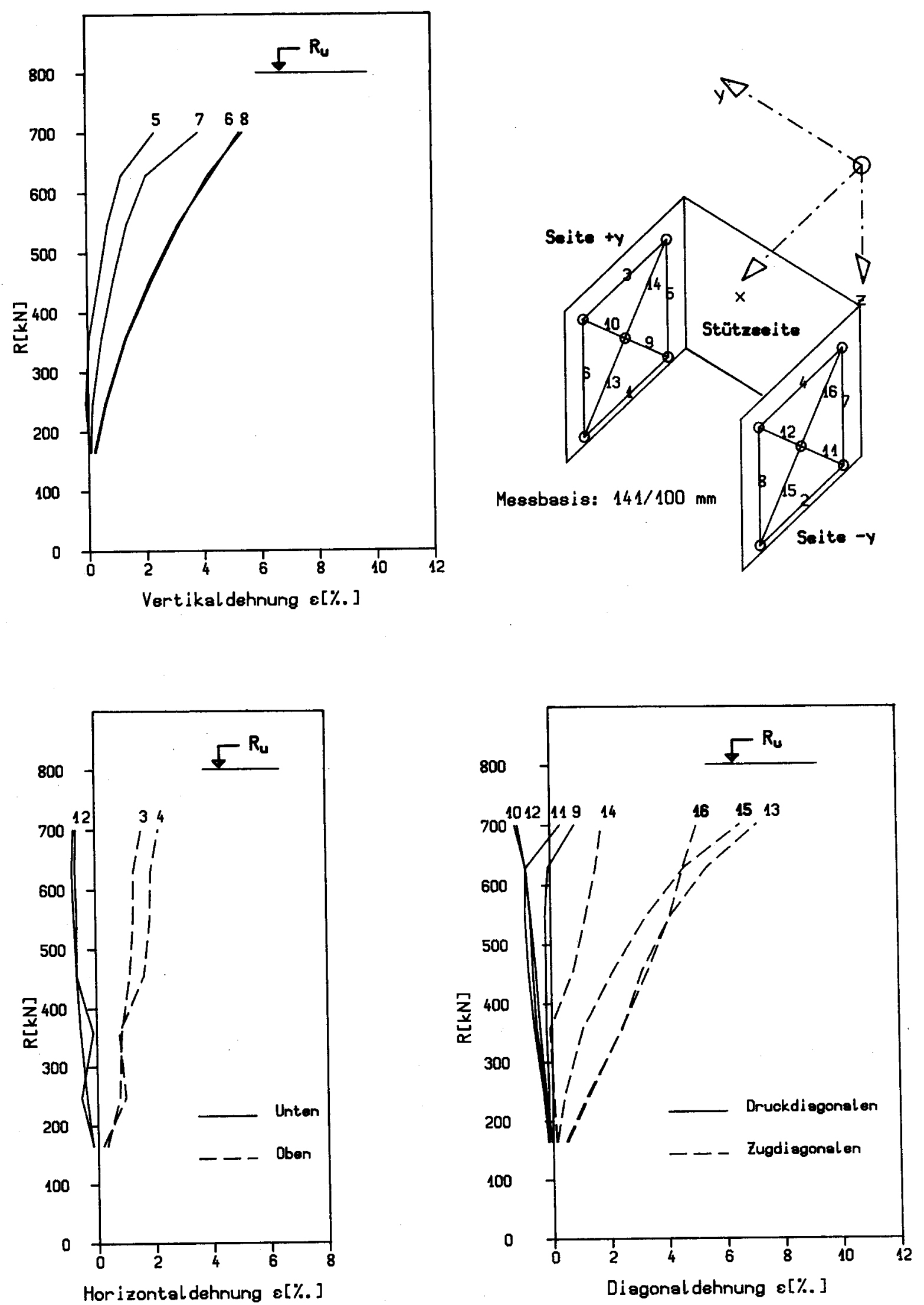

Dehnungen in Funktion der Last, messstellenweise 


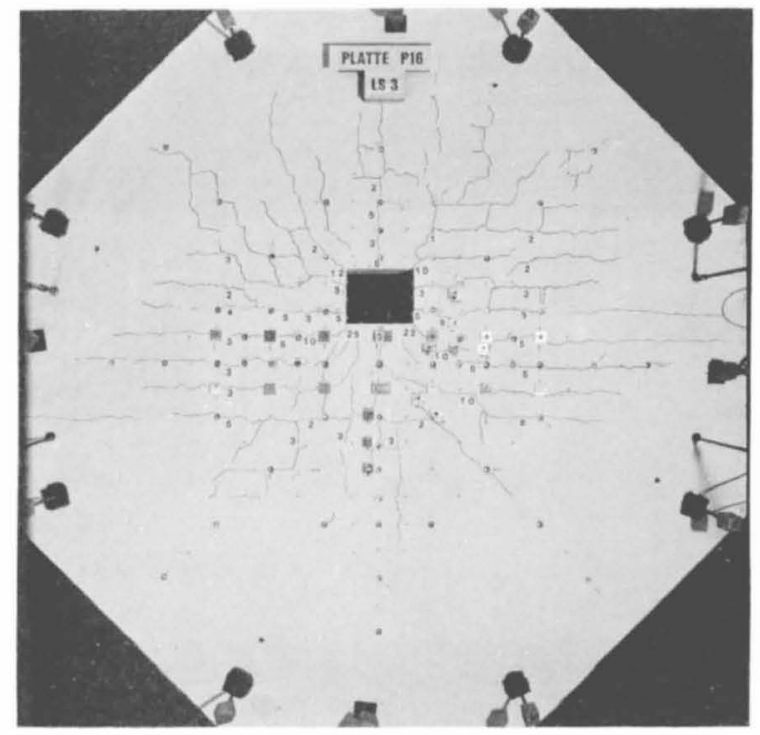

a) Laststufe 3

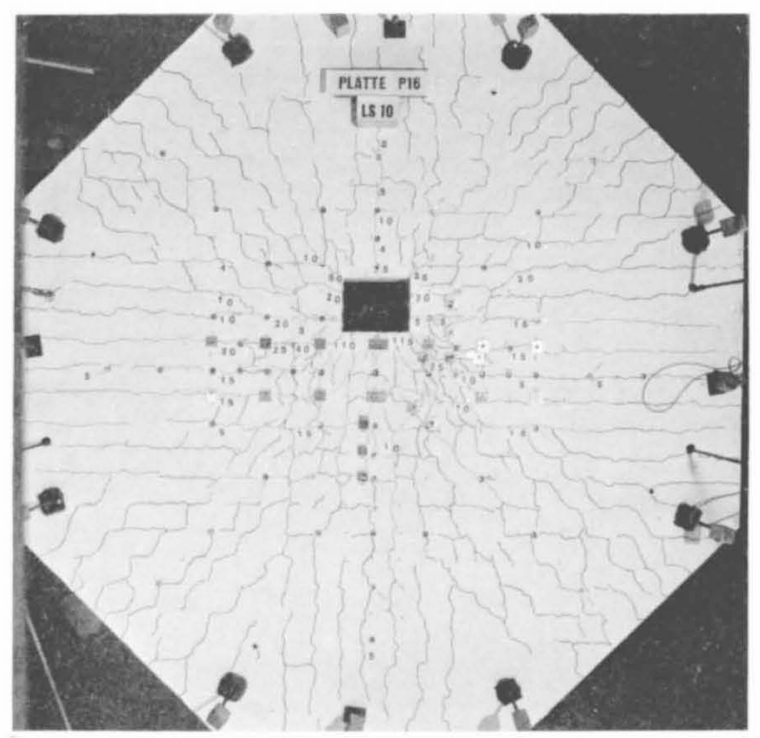

c) Laststufe 10

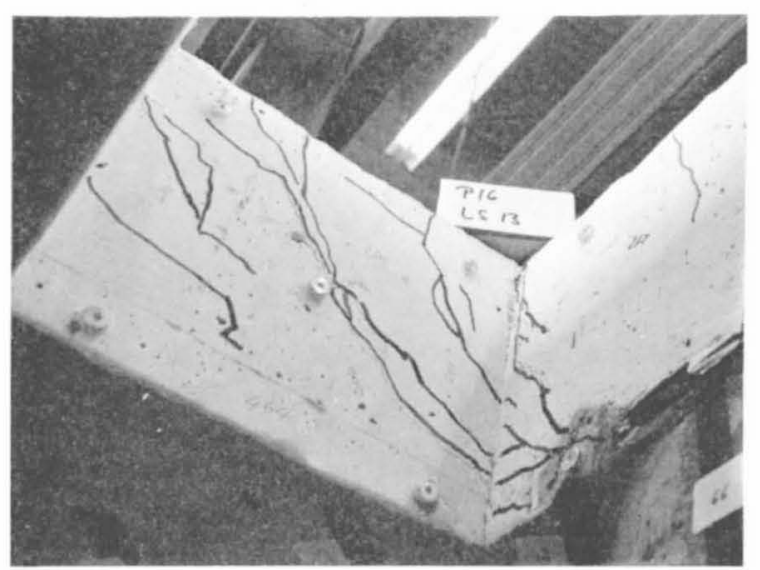

e) Aussparung, Laststufe 13

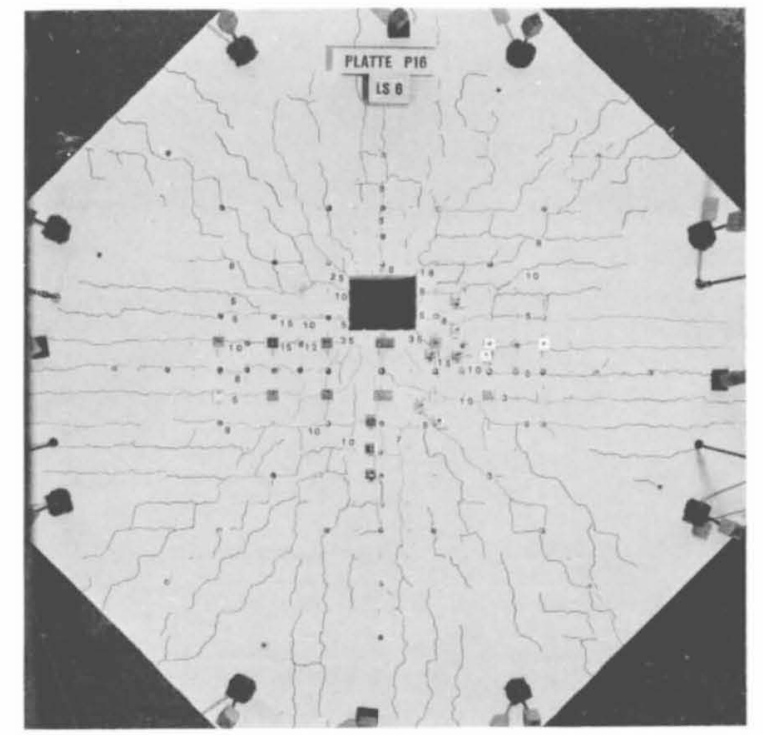

b) Laststufe 6

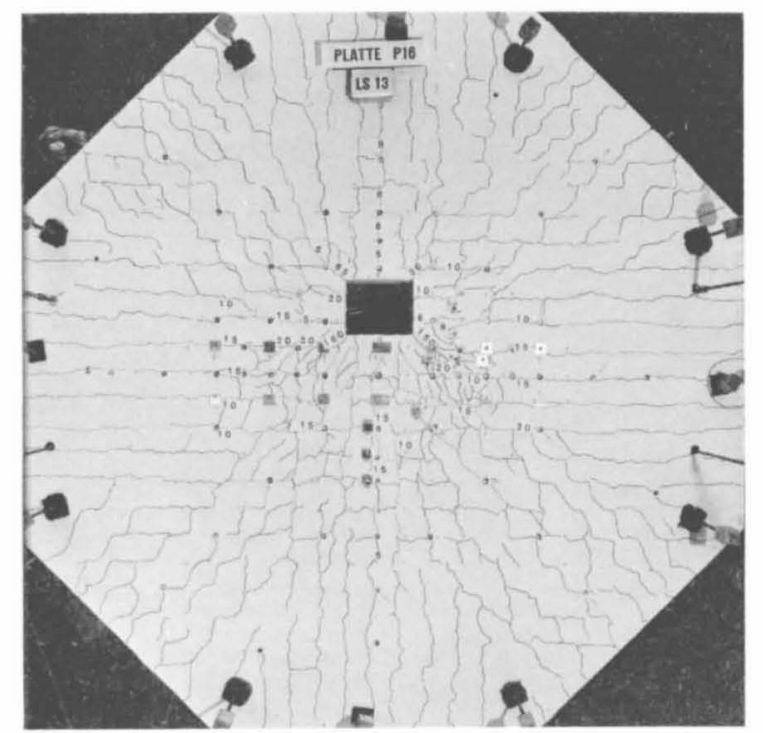

d) Laststufe 13

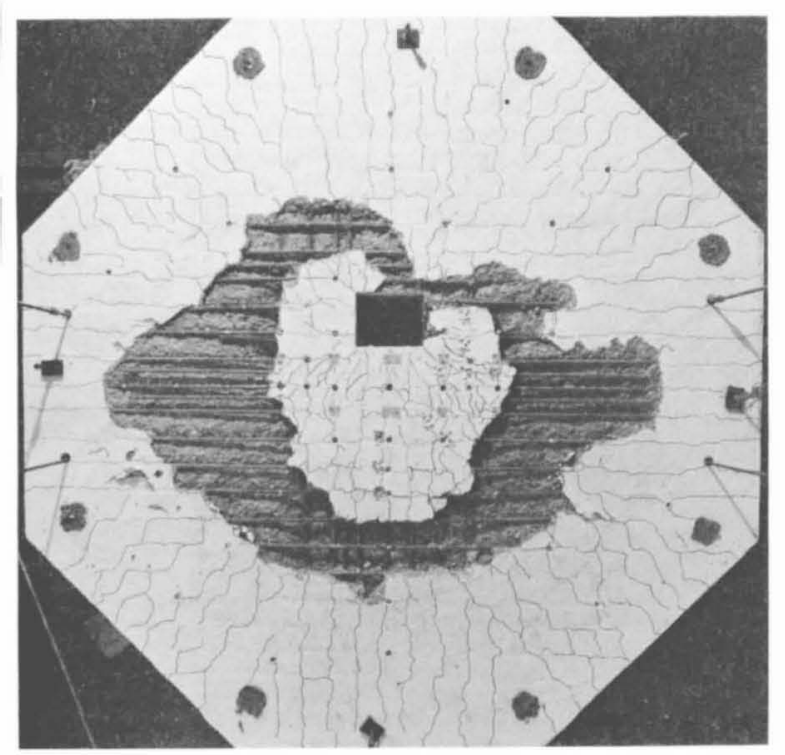

f) Bruch 

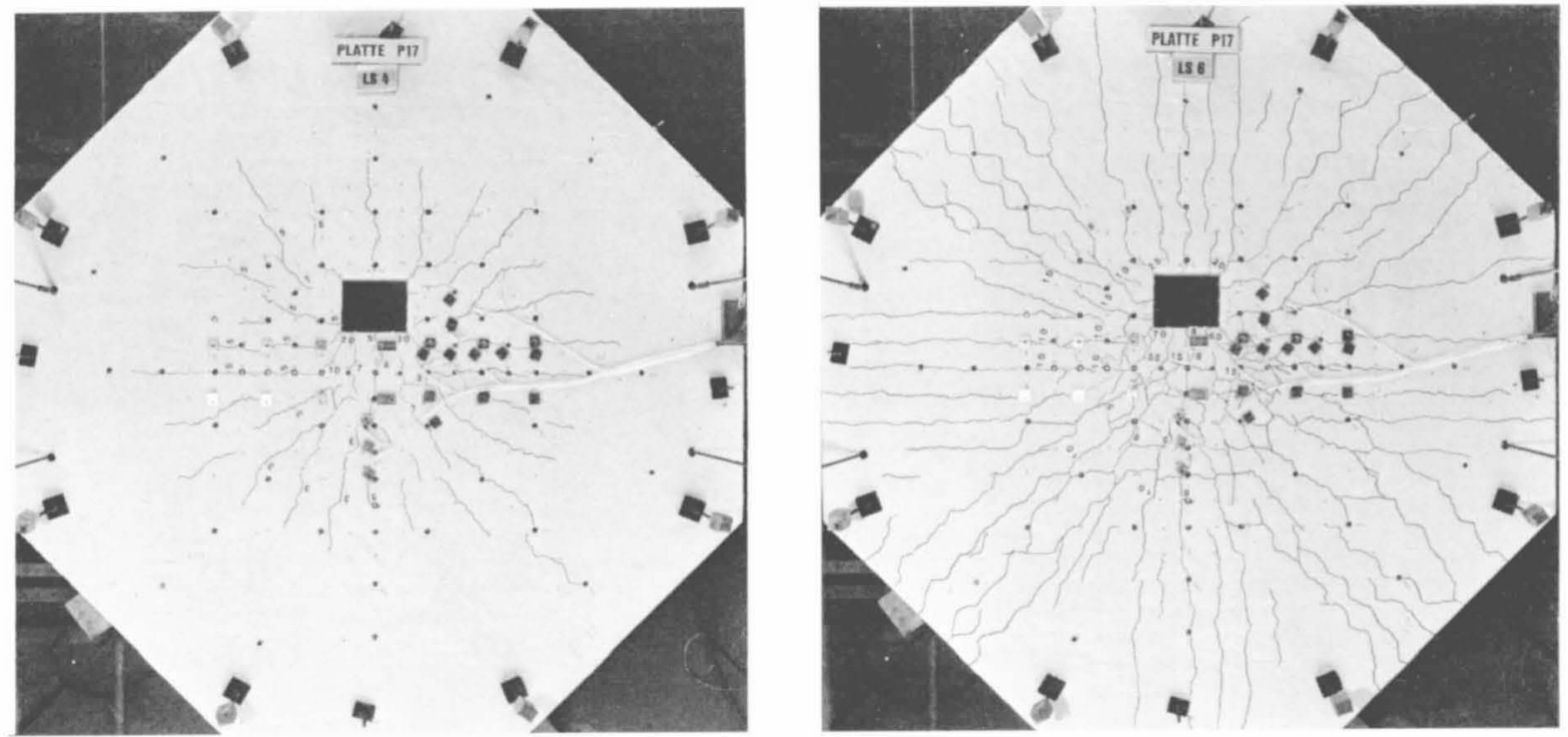

a) Laststufe 4

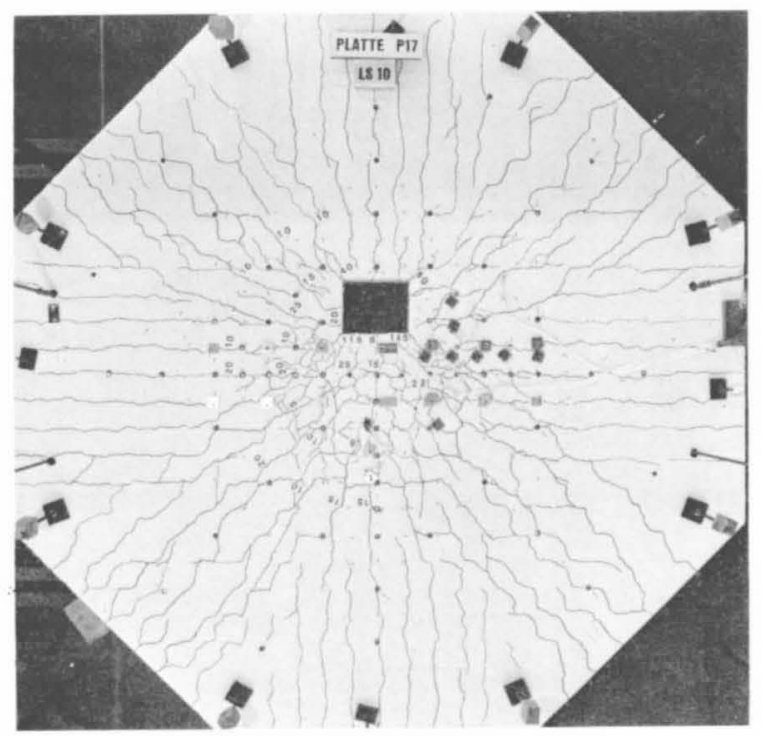

b) Laststufe 6

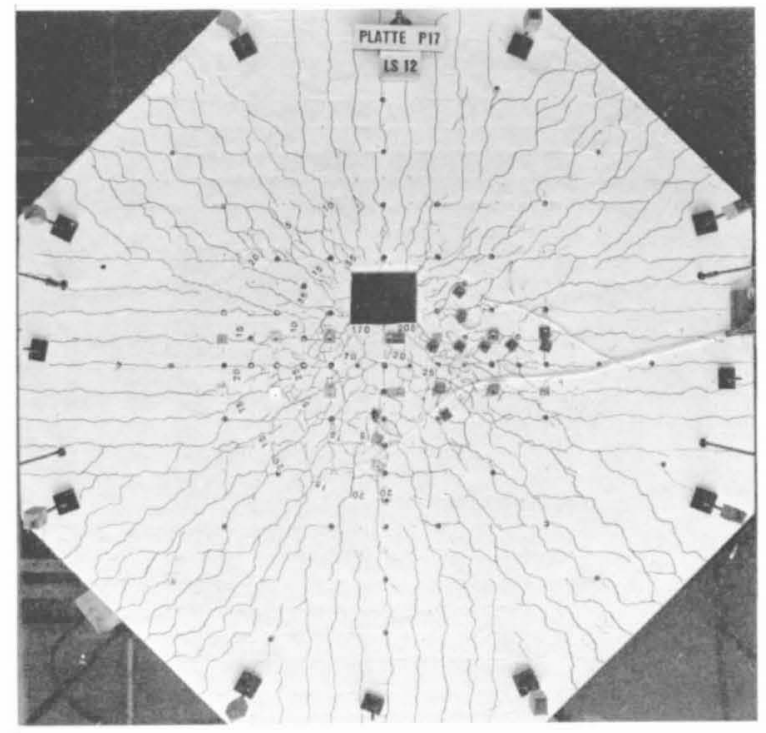

c) Laststufe 10

d) Laststufe 12
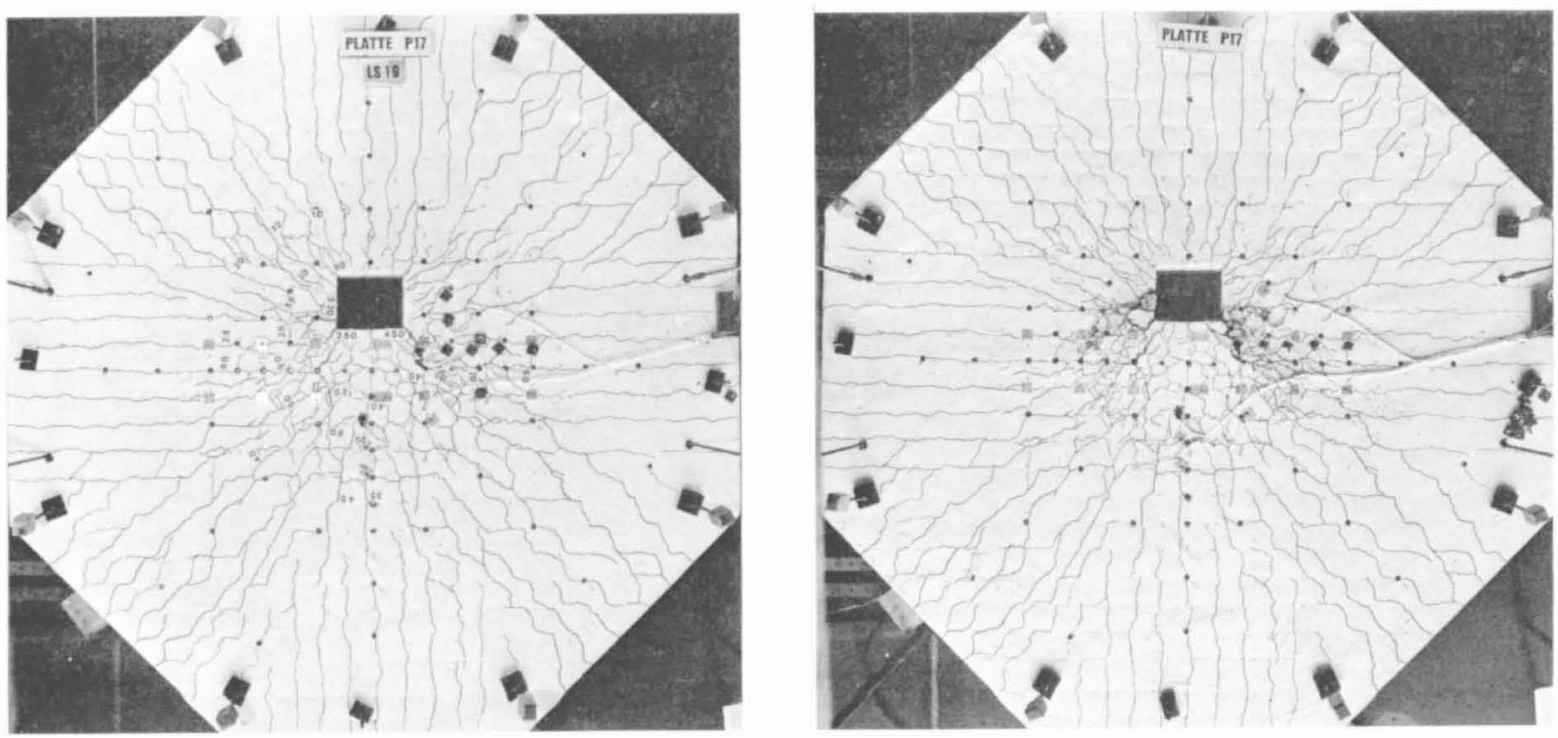

e) Laststufe 19

f) Bruch

Bild 84: Rissentwicklung, Platte P 17 


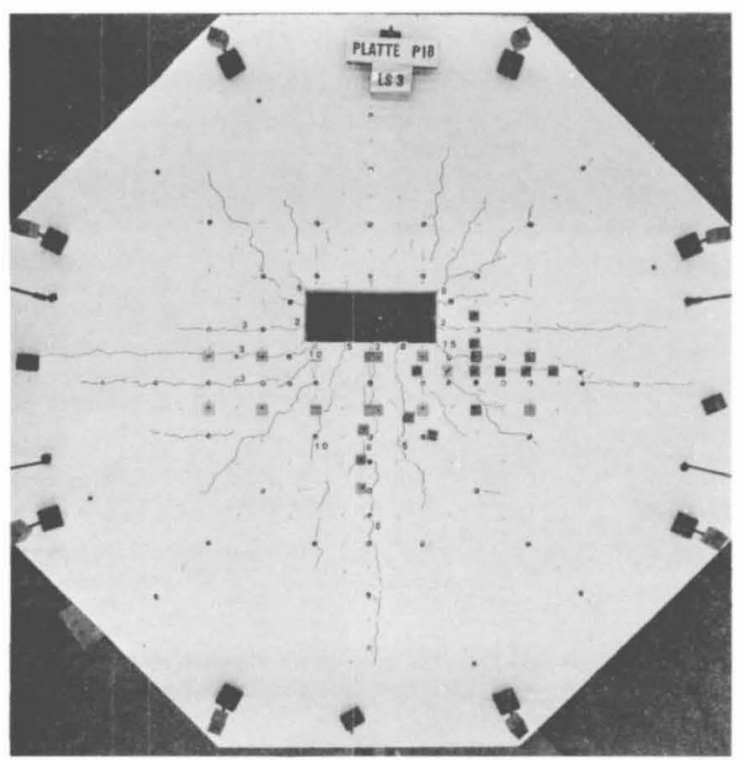

a) Laststufe 3

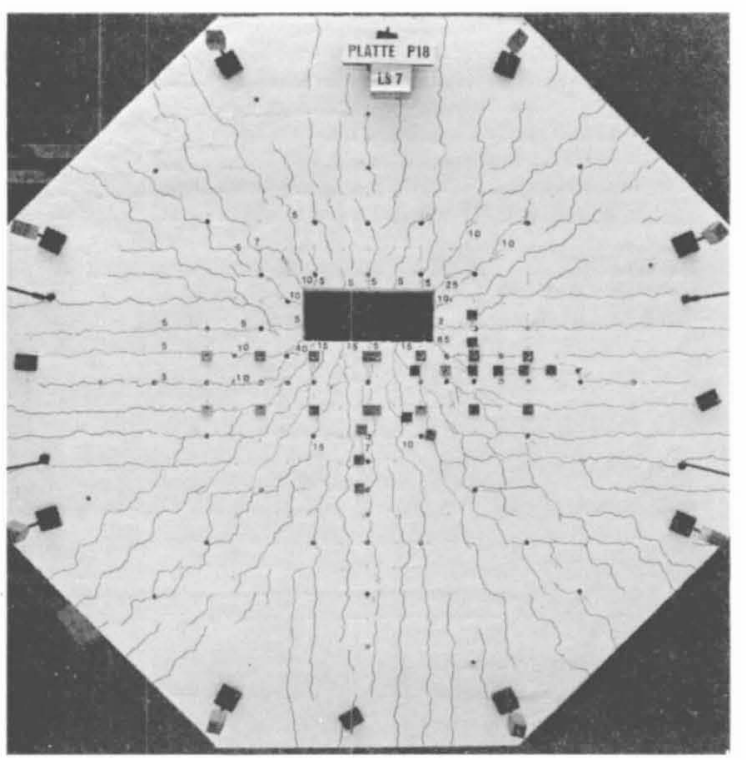

c) Laststufe 7

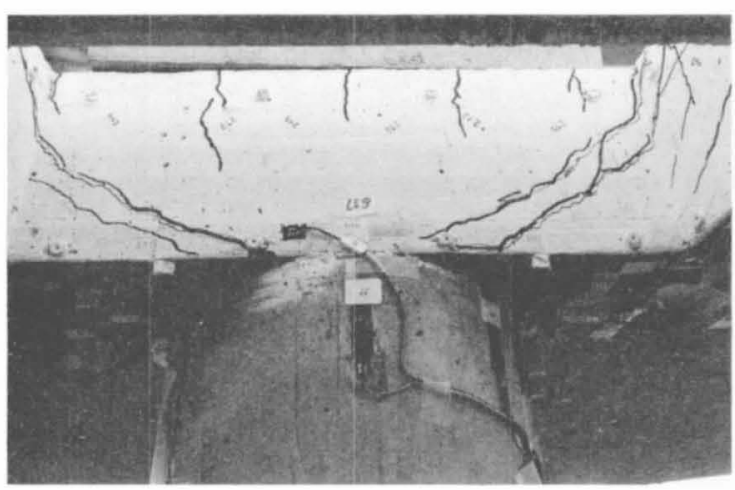

e) Aussparung, Laststufe 9

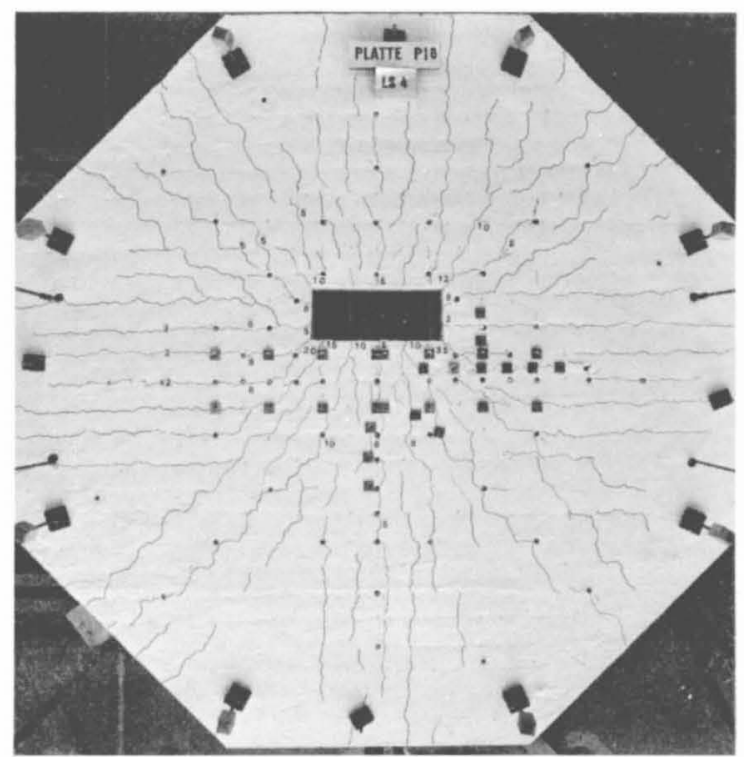

b) Laststufe 4

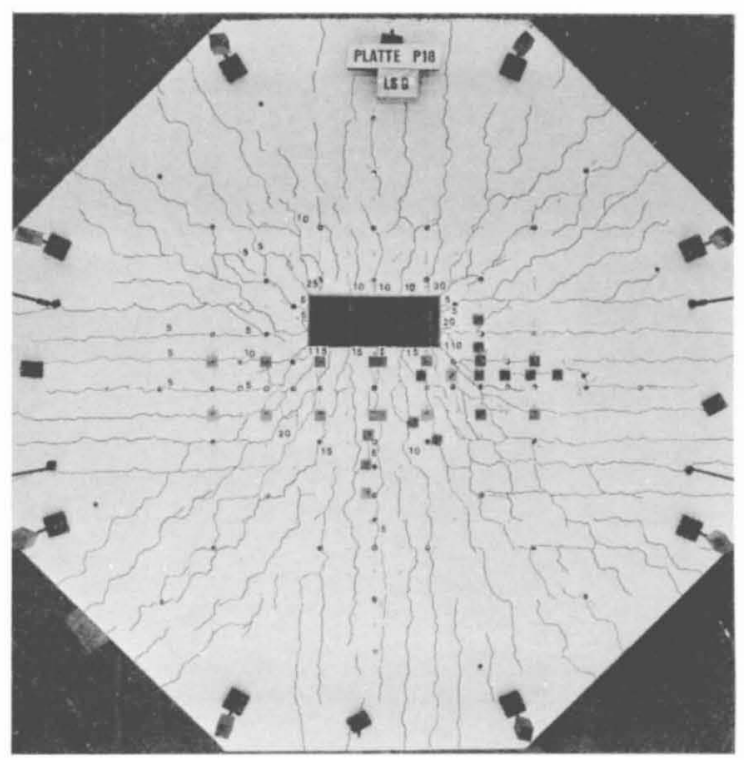

d) Laststufe 9

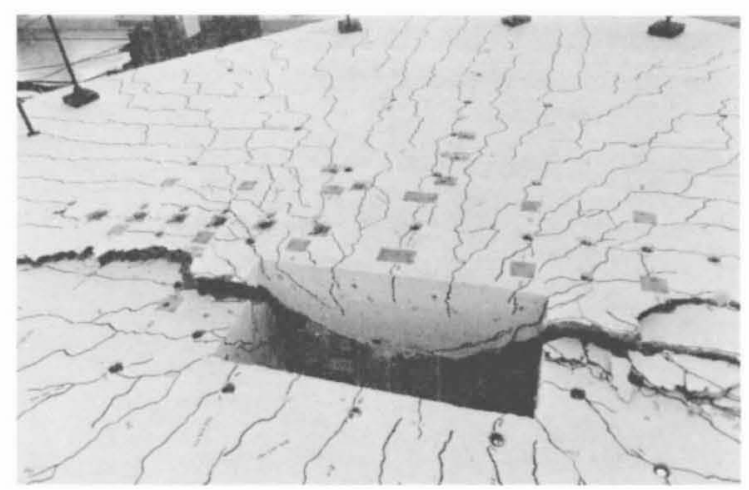

f) Bruch

Bild 85: Rissentwicklung, Platte P 18 


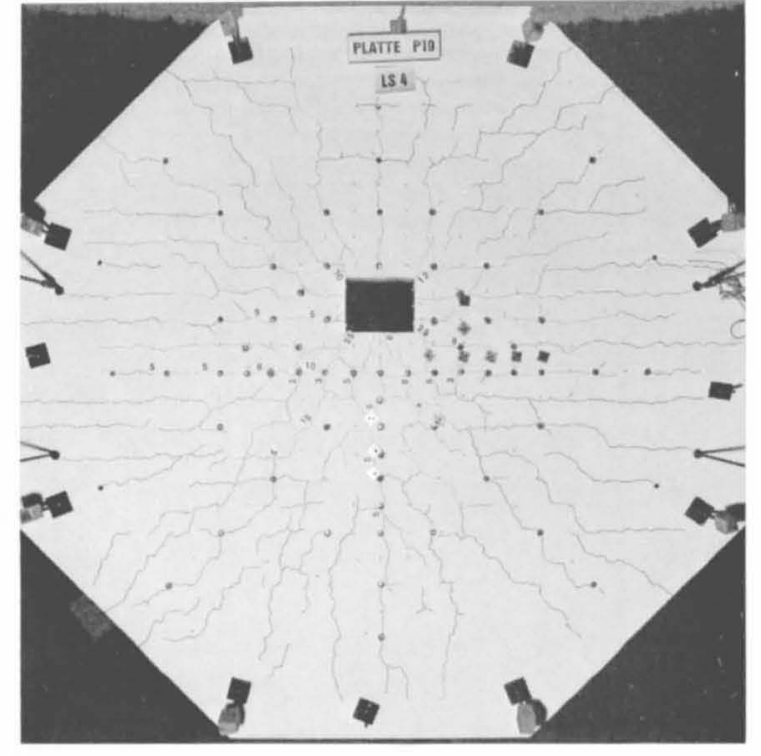

a) Laststufe 4

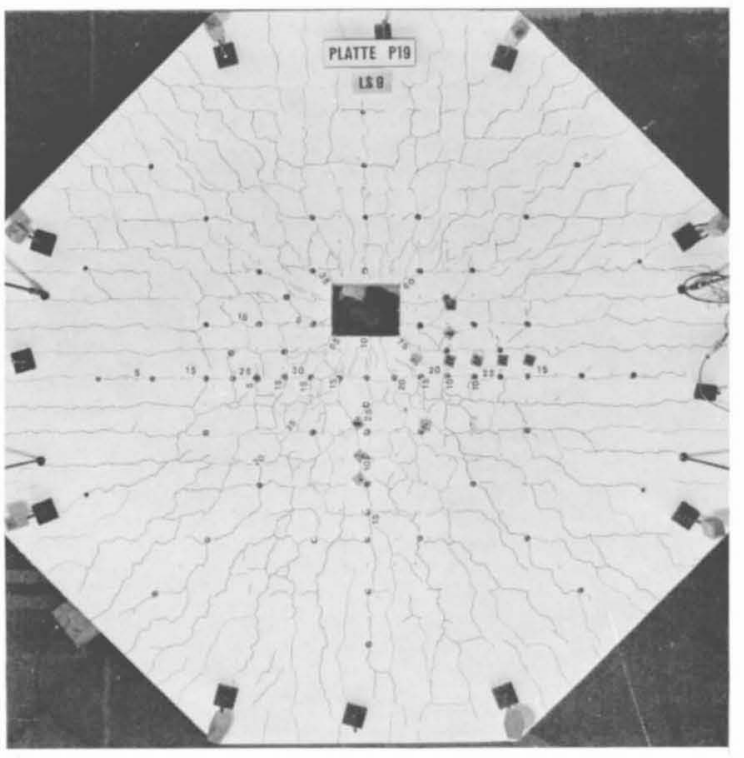

c) Laststufe 9

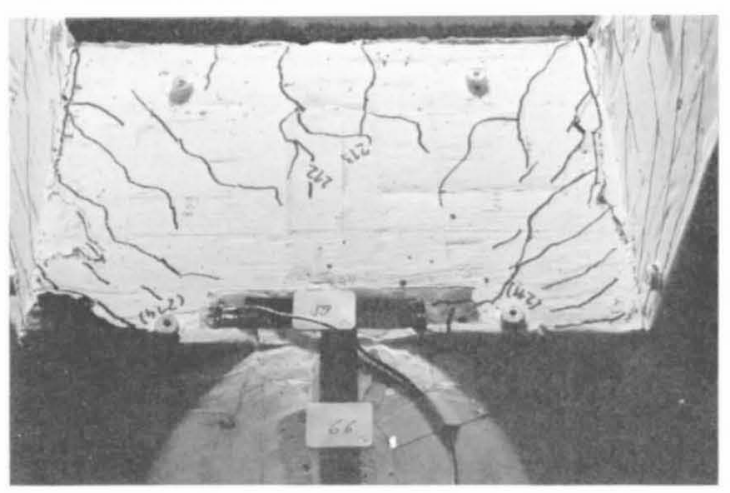

e) Aussparung, Laststufe 12

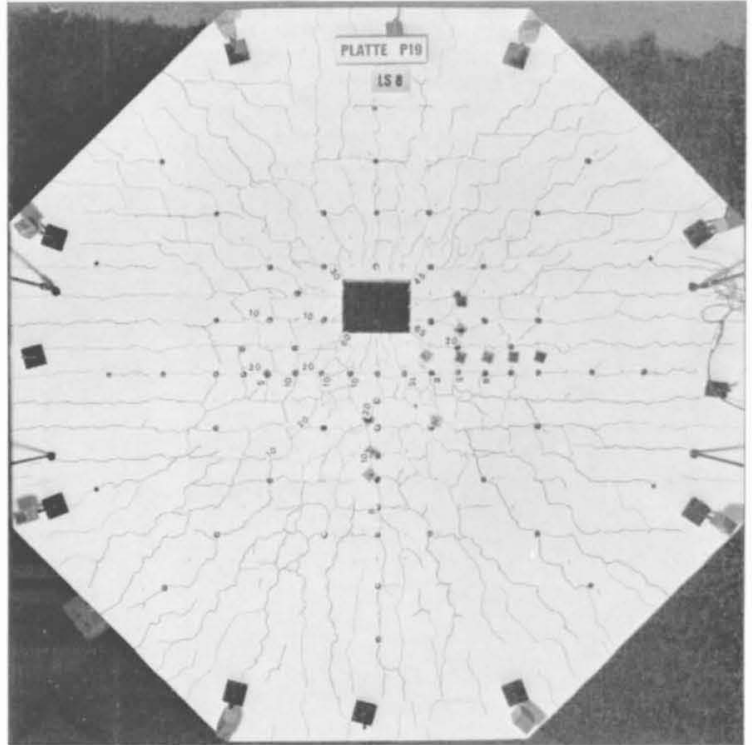

b) Laststufe 8

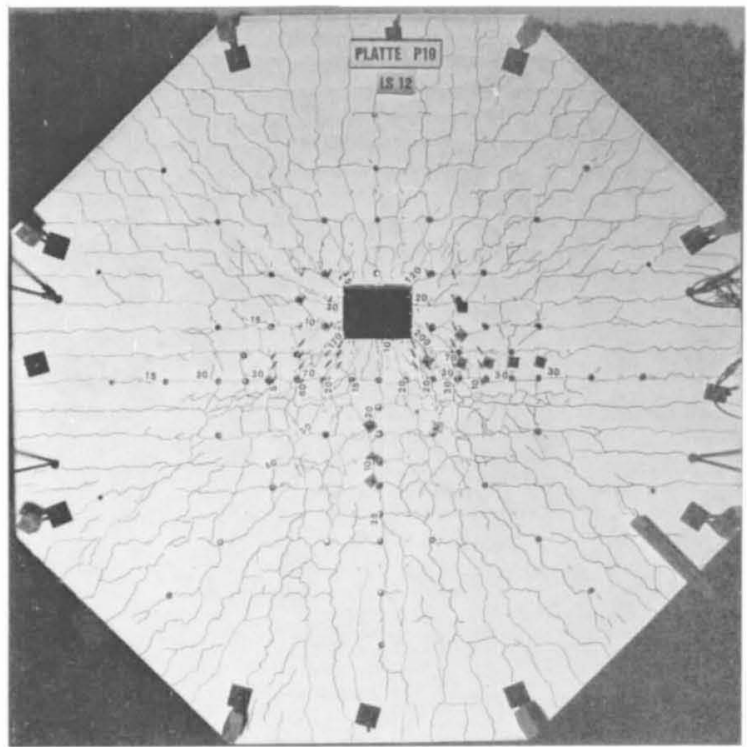

d) Laststufe 12

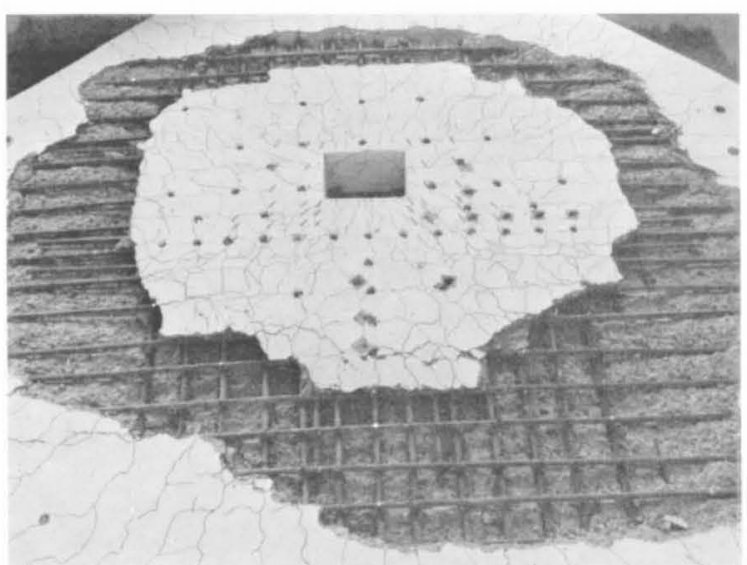

f) Bruch 

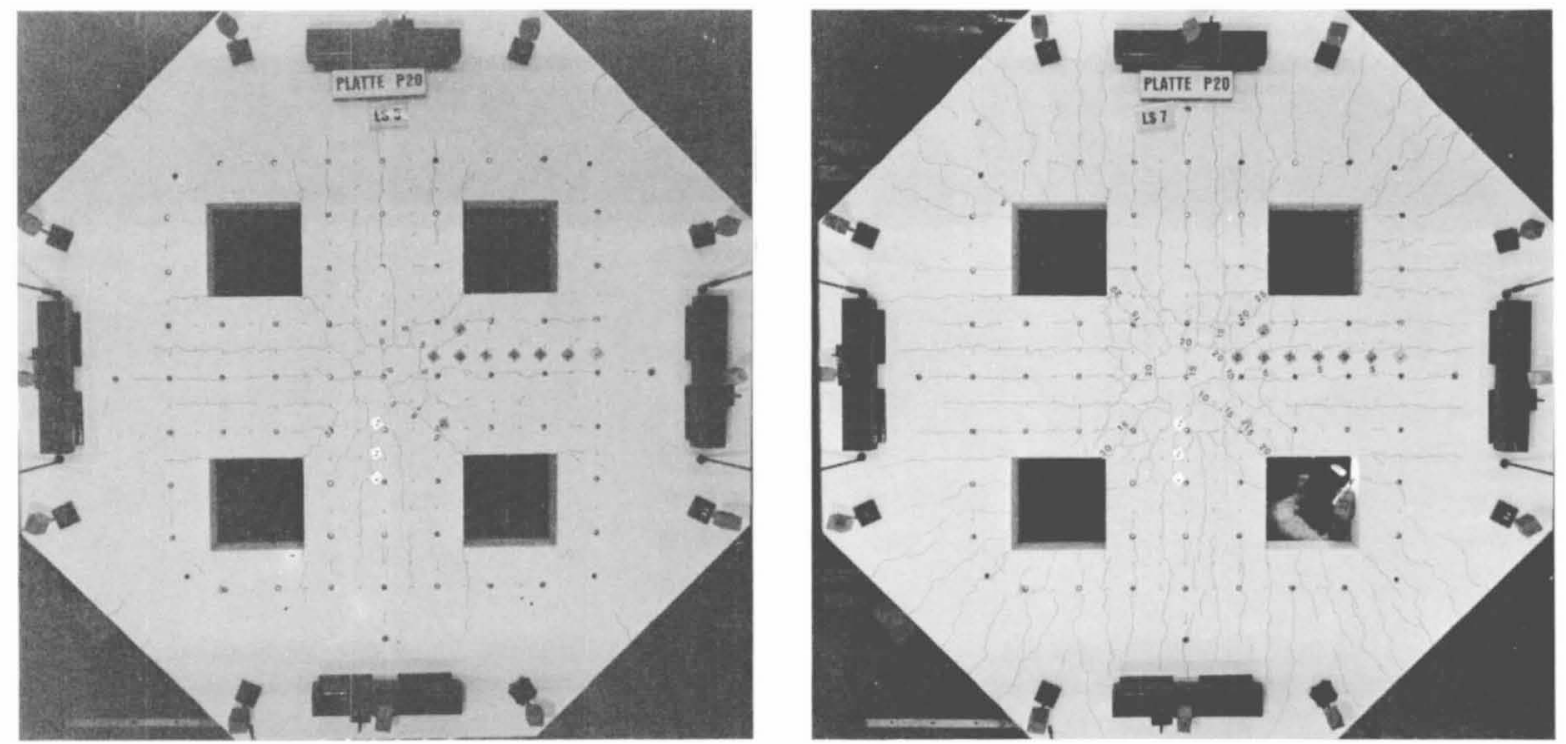

a) Laststufe 5

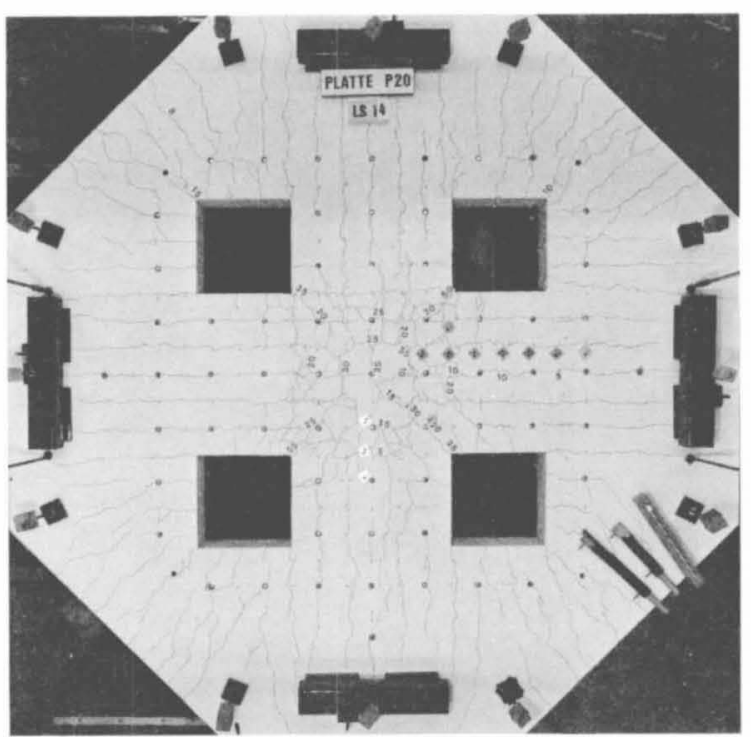

b) Laststufe 7

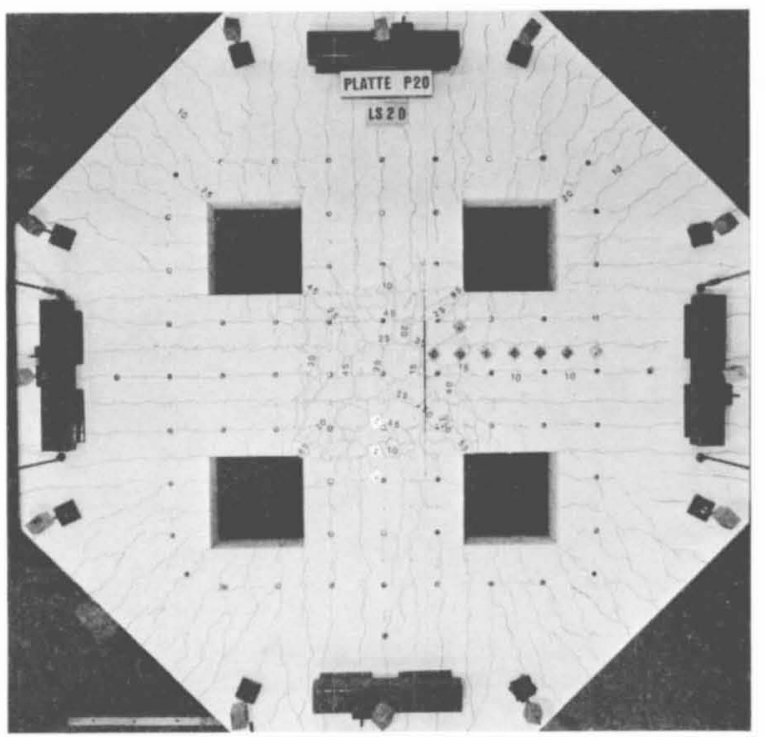

c) Laststufe 14

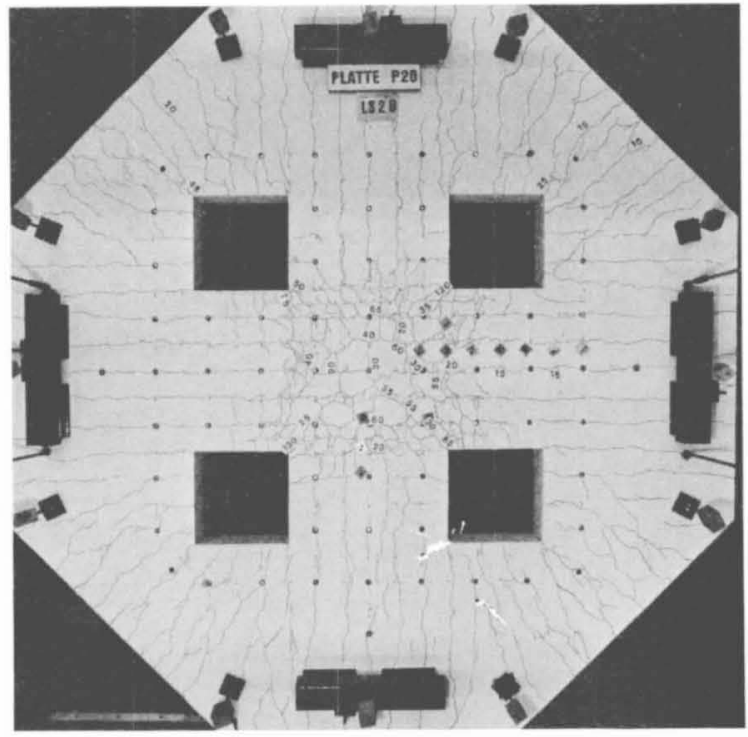

d) Laststufe 20

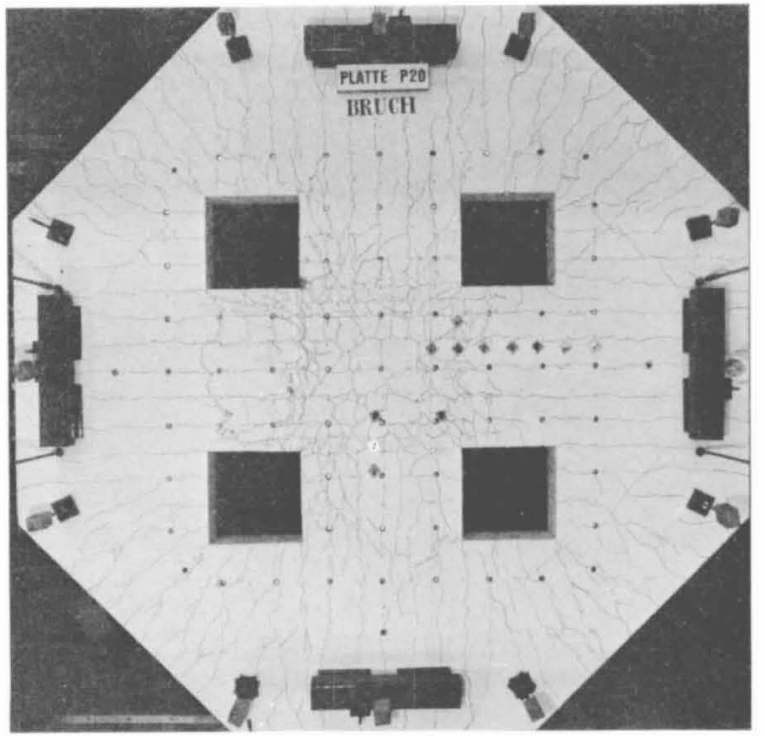

e) Laststufe 26

f) Bruch

Bild 87: Rissentwicklung, Platte P 20 

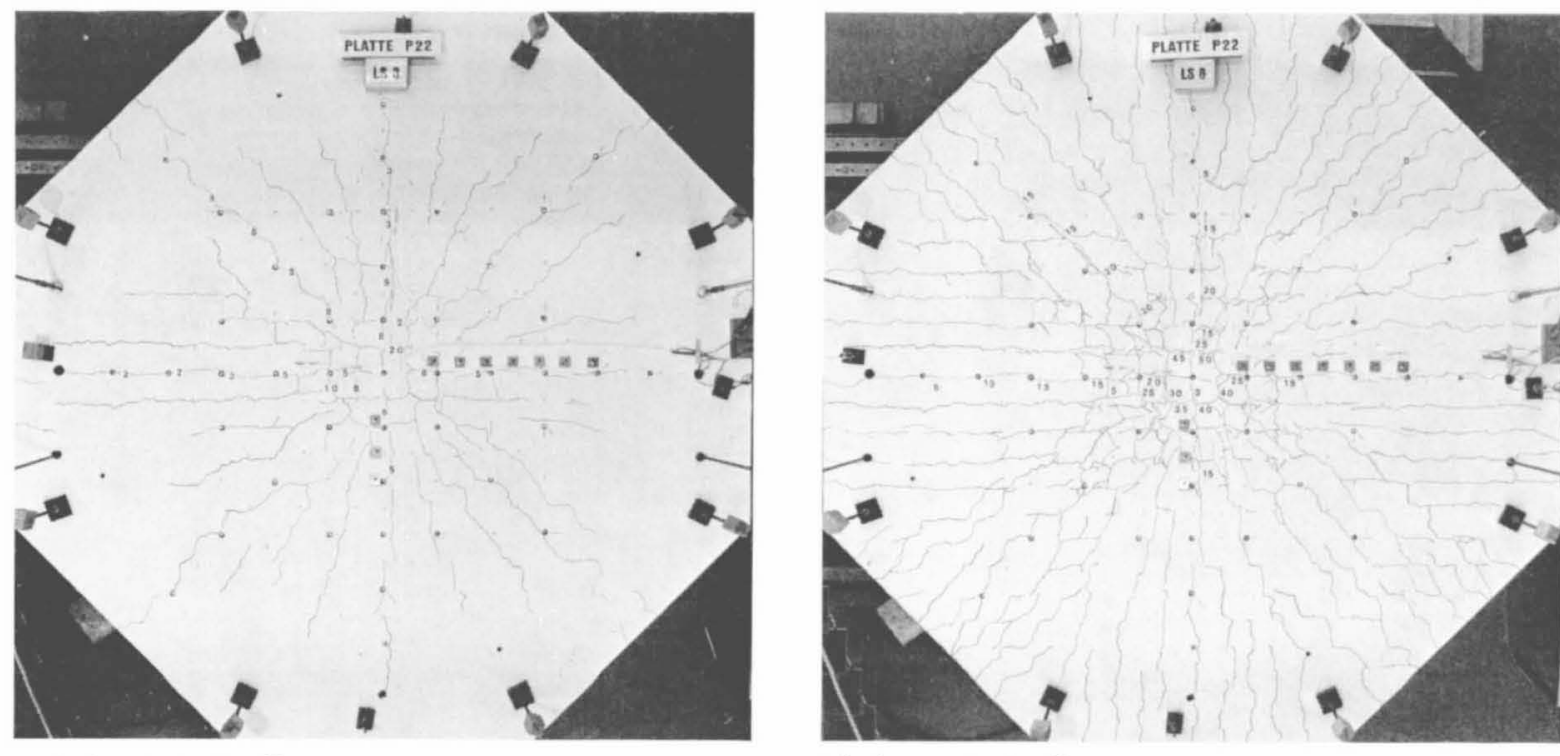

a) Laststufe 3
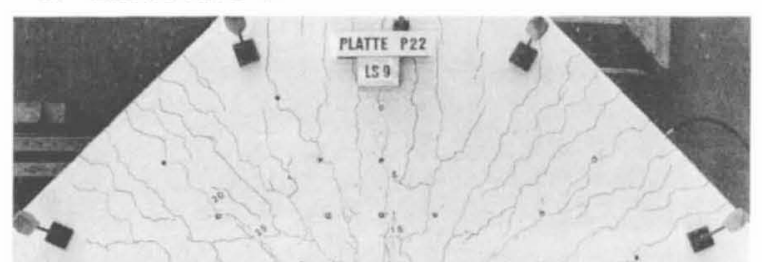

b) Laststufe 8

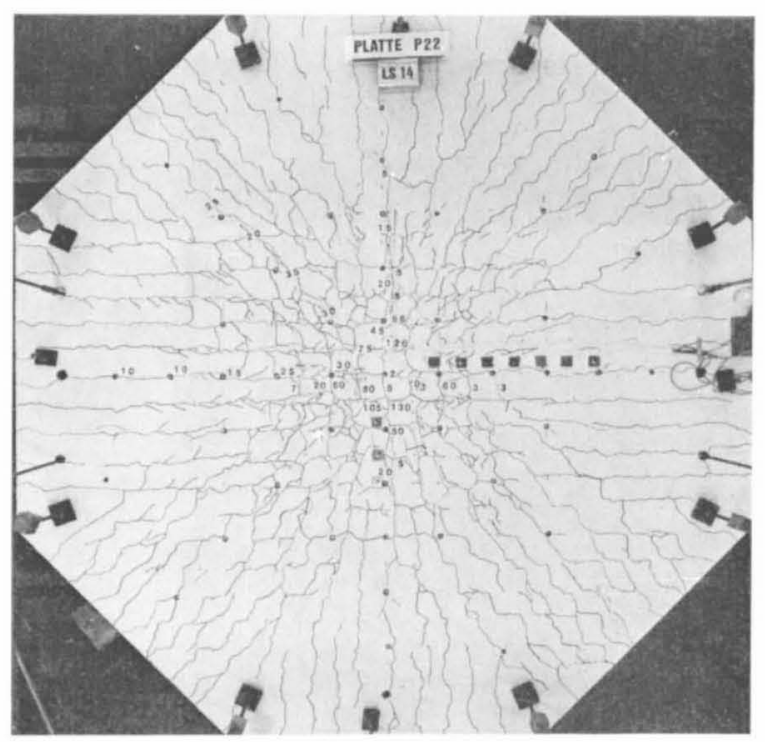

c) Laststufe 9

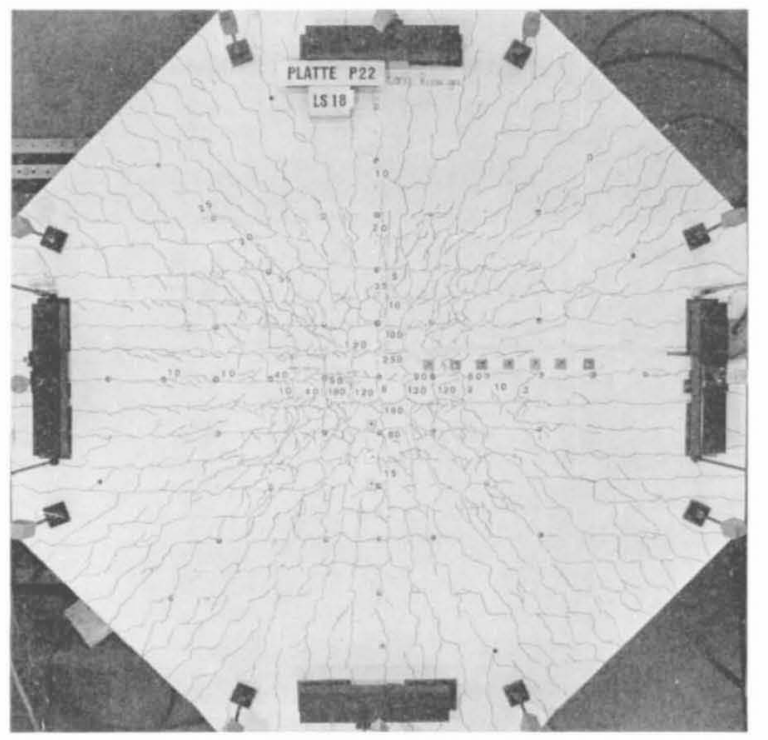

d) Laststufe 14

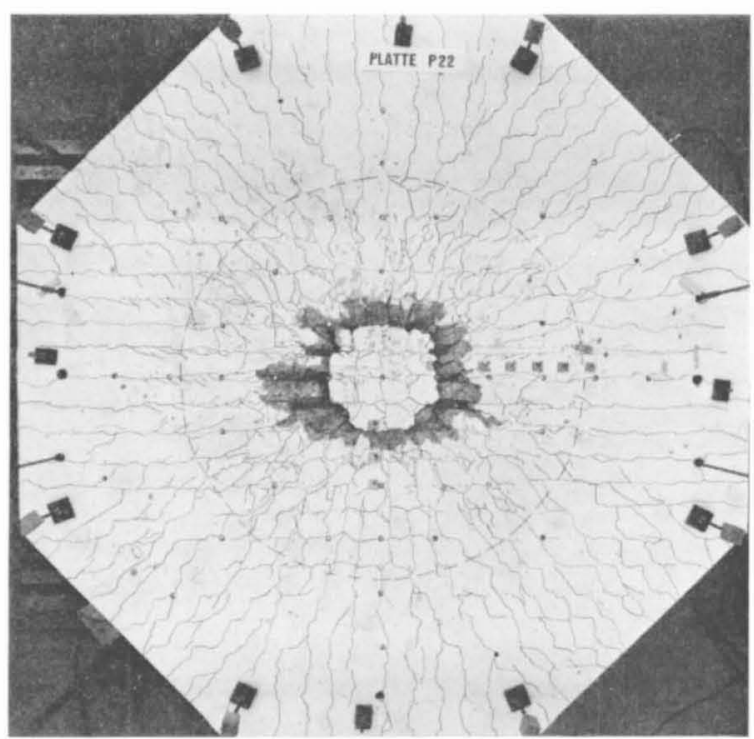

e) Laststufe 18

f) Bruch

Bild 88: Rissentwicklung, Platte P 22 


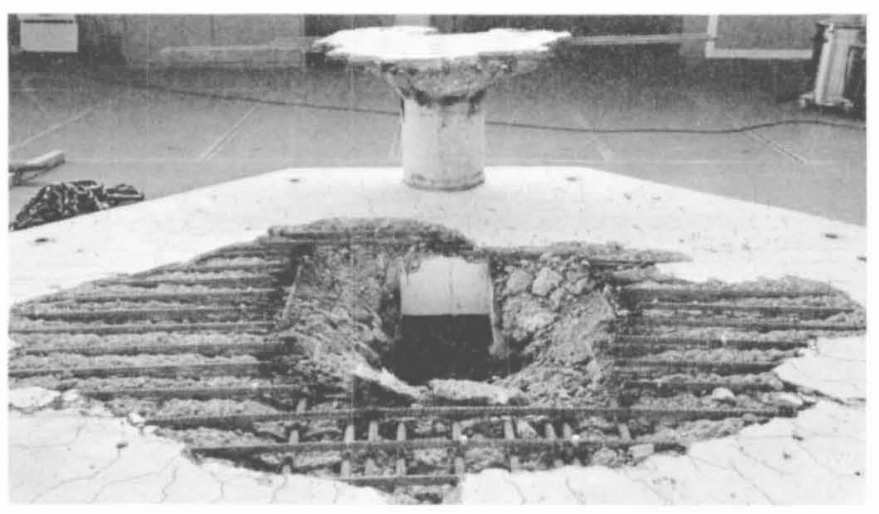

a) Platte P 16 mit Durchstanzkege 1

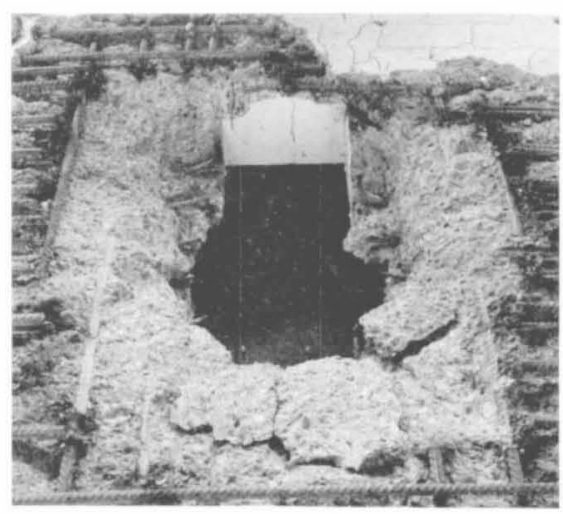

b) Feine Bruchstuicke sind mit dem Staubsauger entfernt worden

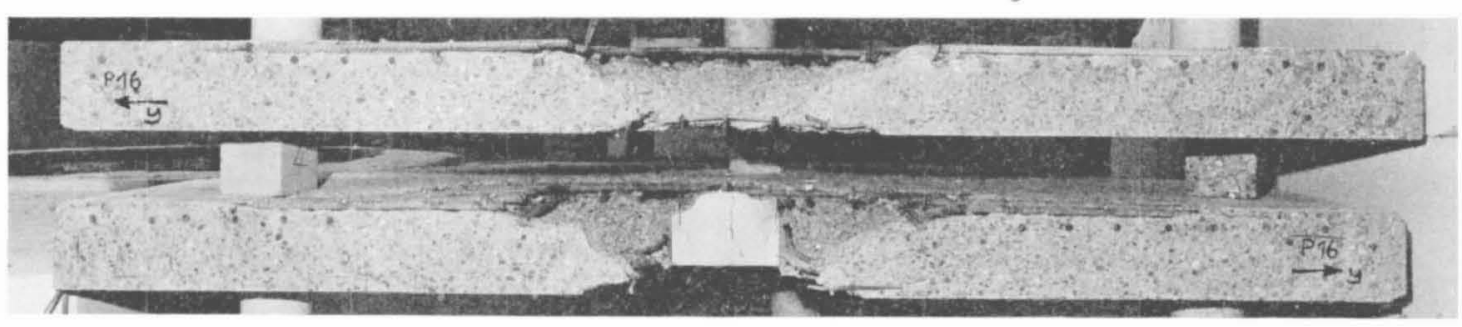

c) Schnitt entlang $x=-110 \mathrm{~mm}$ : Es sind keine diagonalen Risse erkennbar

Bild 89: Bruchbilder, Platte P 16

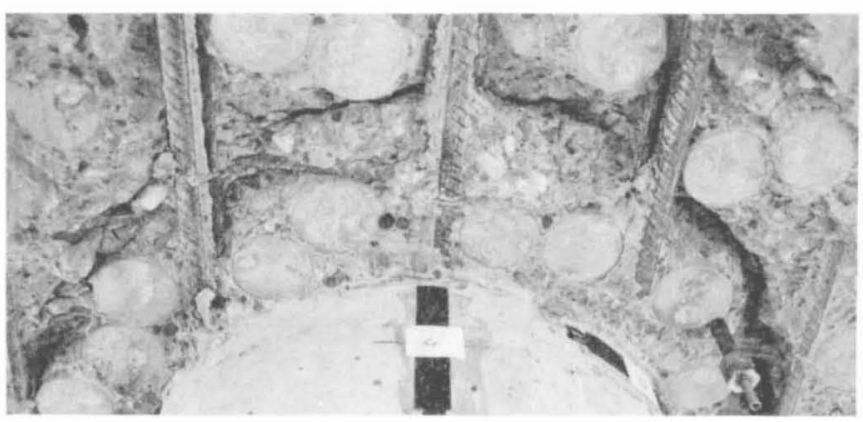

a) Betonüberdeckung an der Unterseite ist abgeplatzt, sehr breiter Riss bei ca. $x=-250 \mathrm{~mm}$

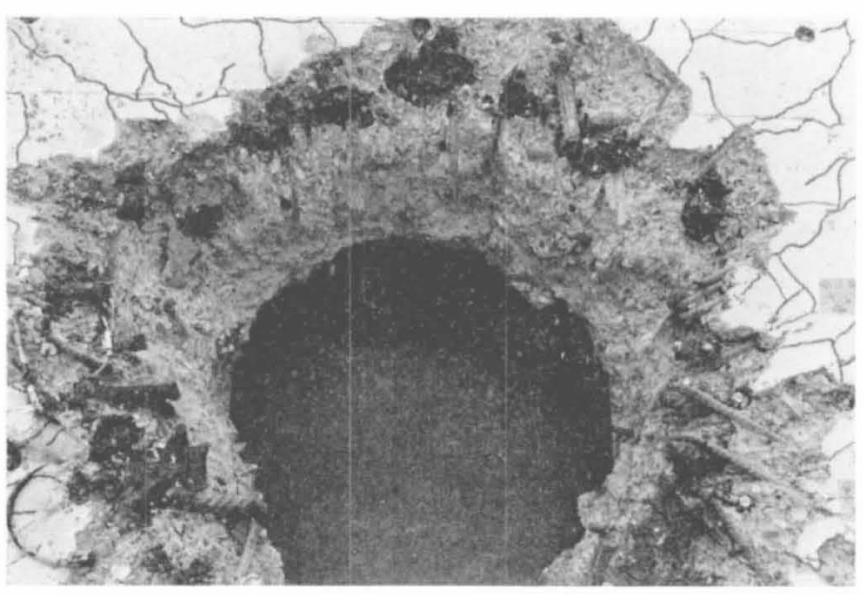

c) Blickrichtung: Negative $x$-Achse

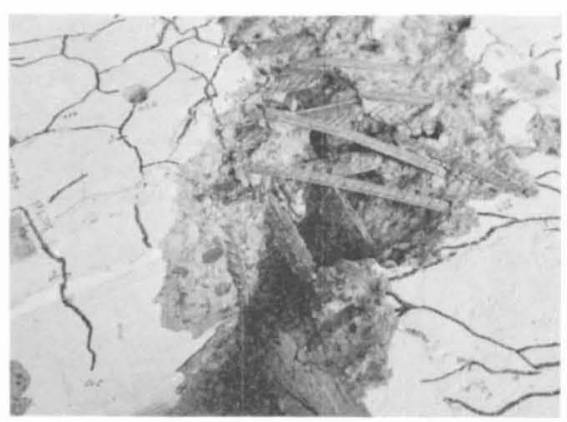

b) Bereich mit starker Schiebung ( $x=150 \mathrm{~mm}$, $y=-125 \mathrm{~mm})$, loser Beton ist entfernt worden

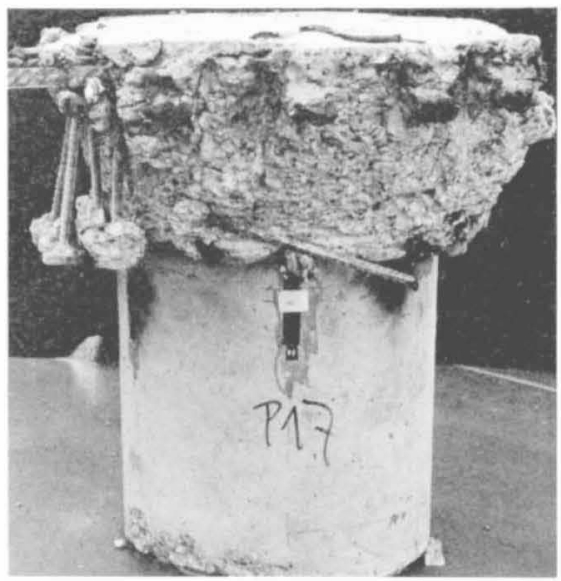

d) Blickrichtung: Positive $x$-Achse

Bild 90: Bruchbilder, Platte P 17 


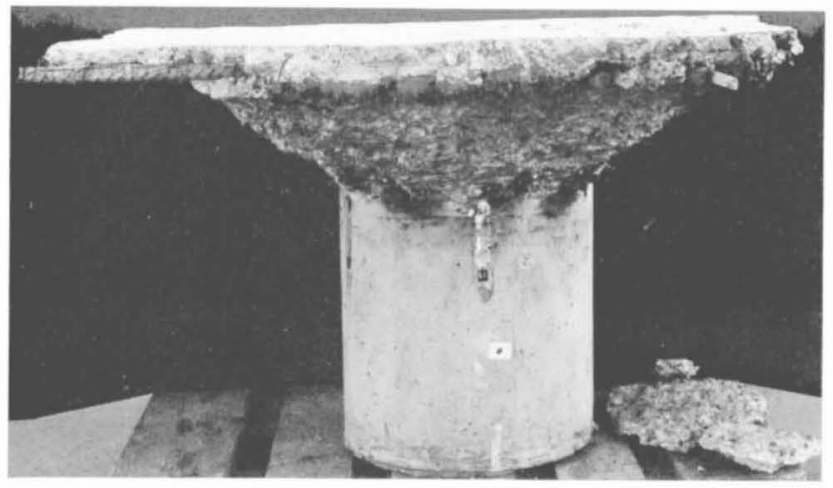

a) Platte P 18 mit Durchstanzkegel

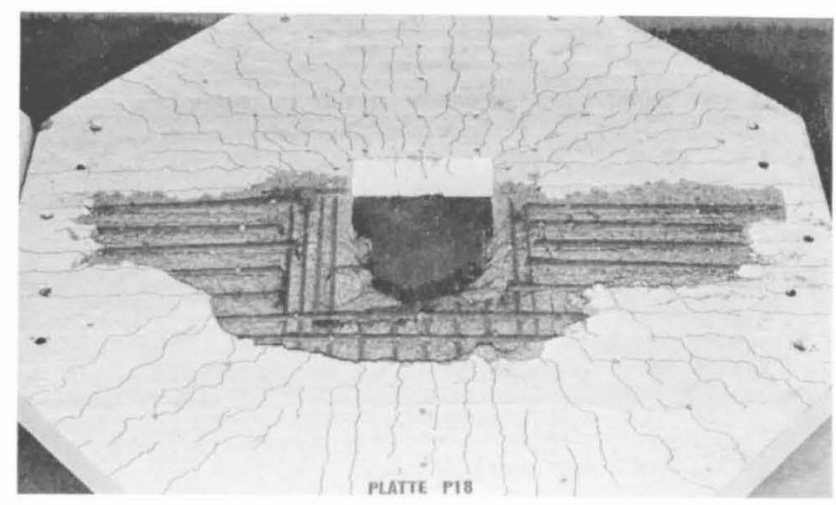

c) Blickrichtung: Positive $x$-Achse

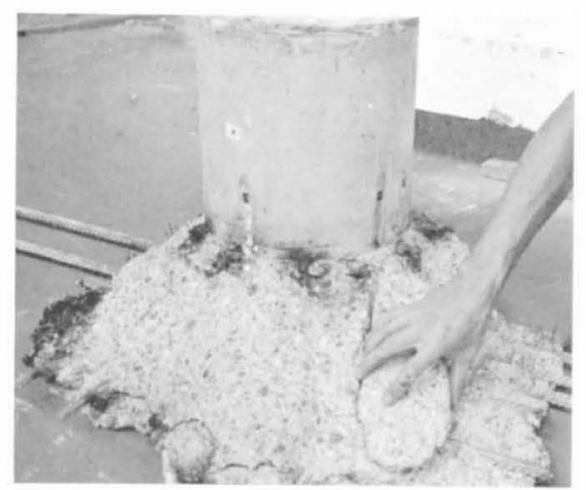

b) Durchstanzkege1 mit losen Betonstücken

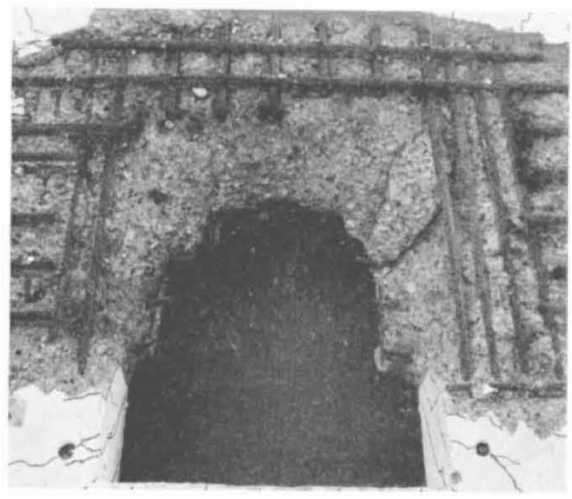

d) Blickrichtung: Negative $x$-Achse

Bild 91: Bruchbilder, Platte P 18

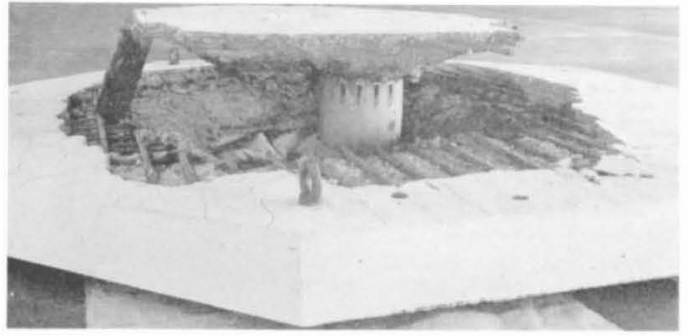

a) Blickrichtung: Positive $y$-Achse

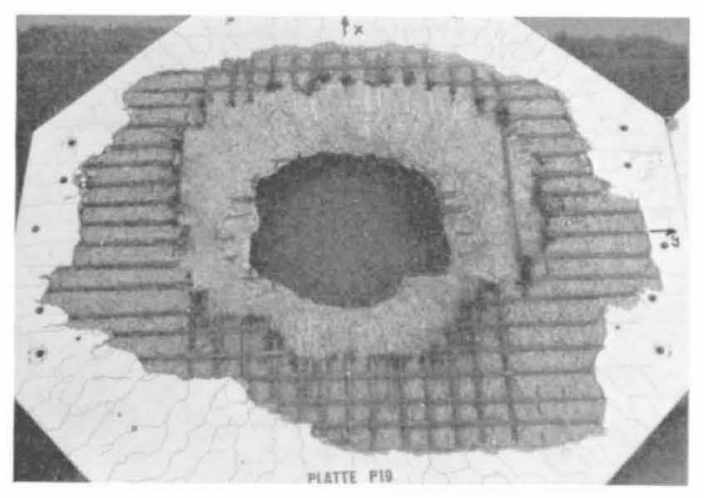

b) Bruchbereich

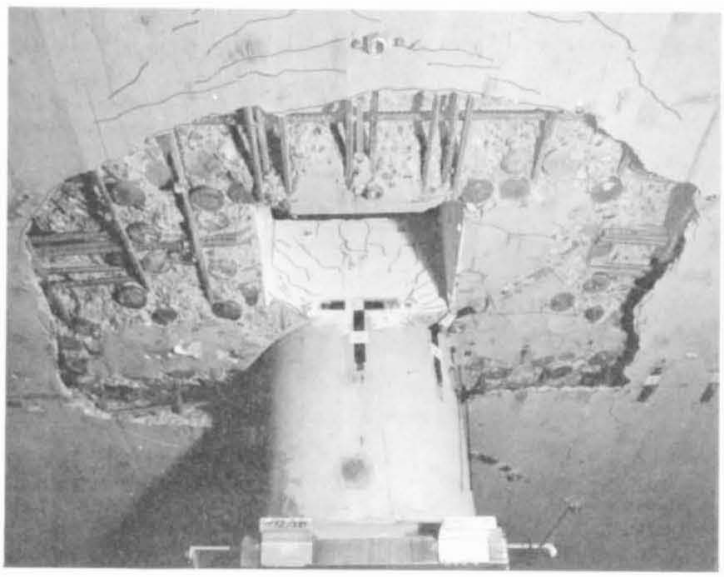

c) Bruchbereich Plattenunterseite; Ankerplättchen der Bügel sind sichtbar infolge Betonabplatzungen

Bild 92: Bruchbilder, Platte P 19 


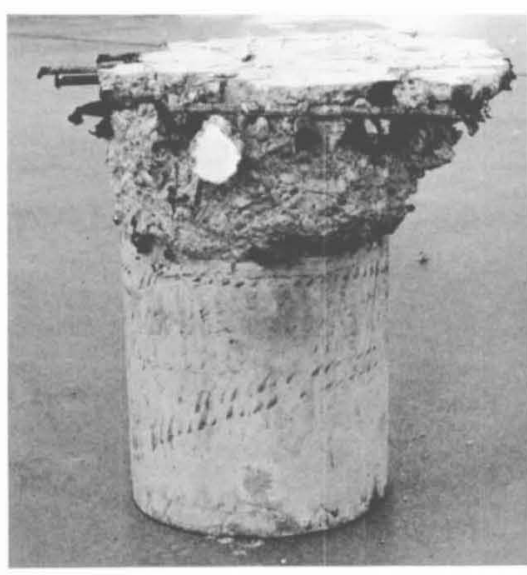

a) Blickrichtung: Positive $x$-Achse

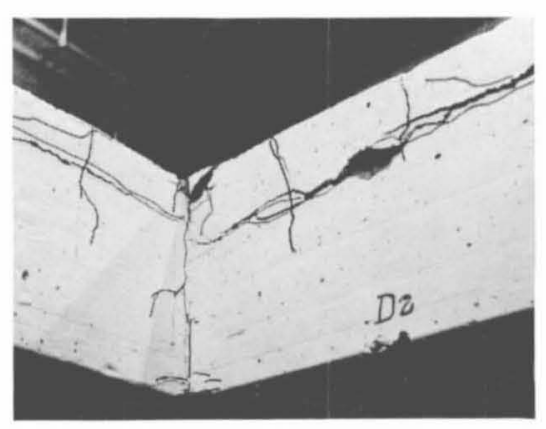

c) Aussparung $-x,-y$,

Blickrichtung gegen Stütze

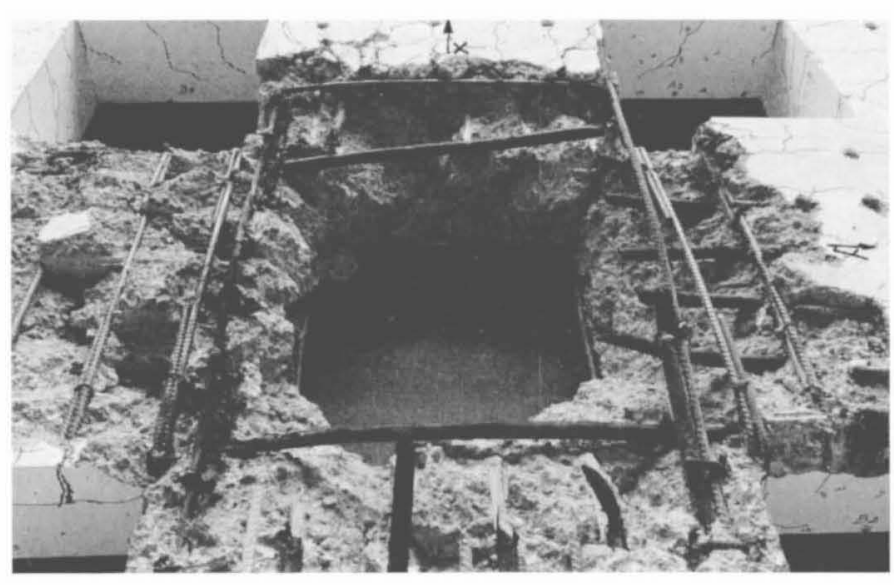

b) Bruchbereich nach Reinigung mit dem Staubsauger

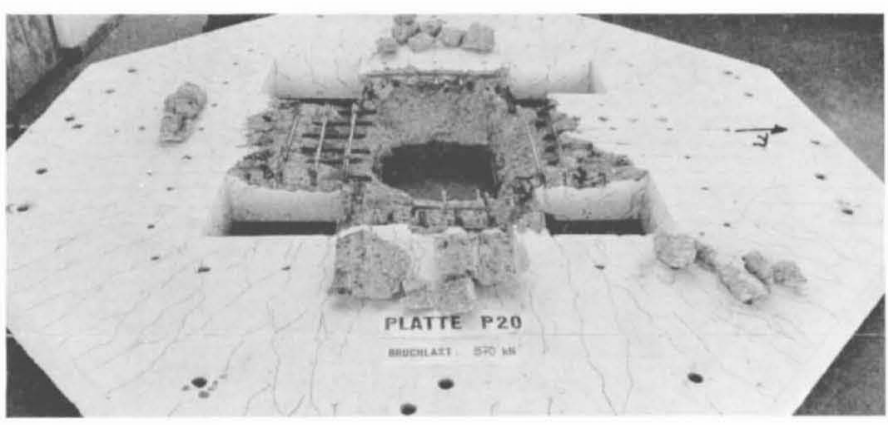

d) grössere lose Betonstïcke sind aus dem Bruchbereich entfernt und auf die Platte gelegt worden

Bild 93: Bruchbilder, Platte P 20

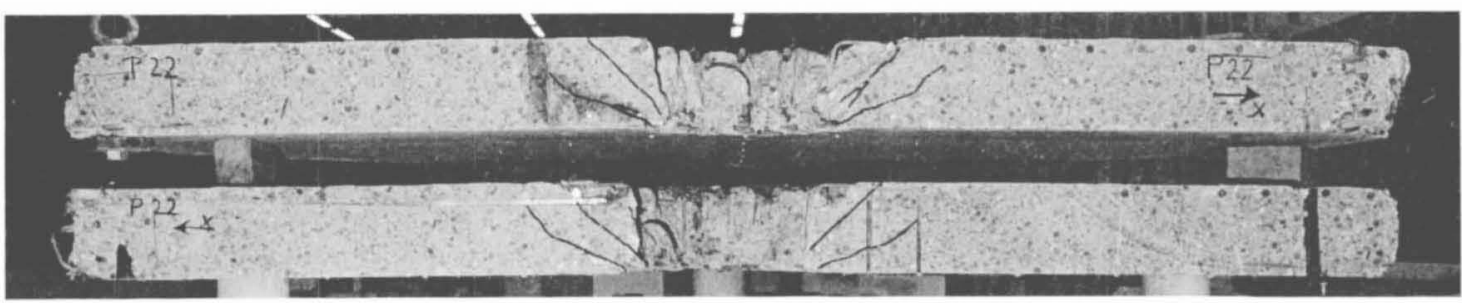

a) Schnitt entlang von $y=0$; die Diagonalrisse sind nachgezeichnet

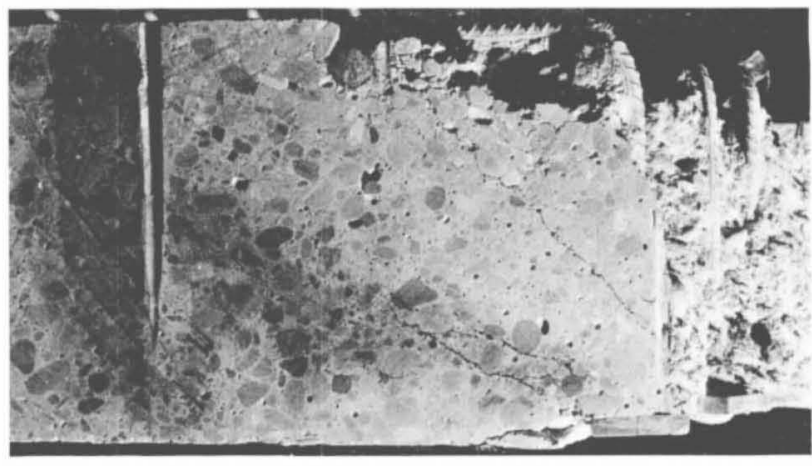

b) Ausschnitt aus Bild 94a (obere Platte)

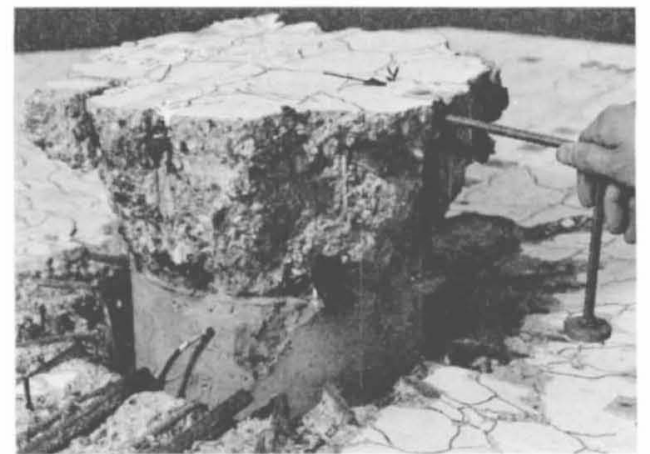

c) Durchstanzkegel mit einem Bügel des innersten Ringes in Soll-Lage

Bild 94: Bruchbilder, Platte P 22 

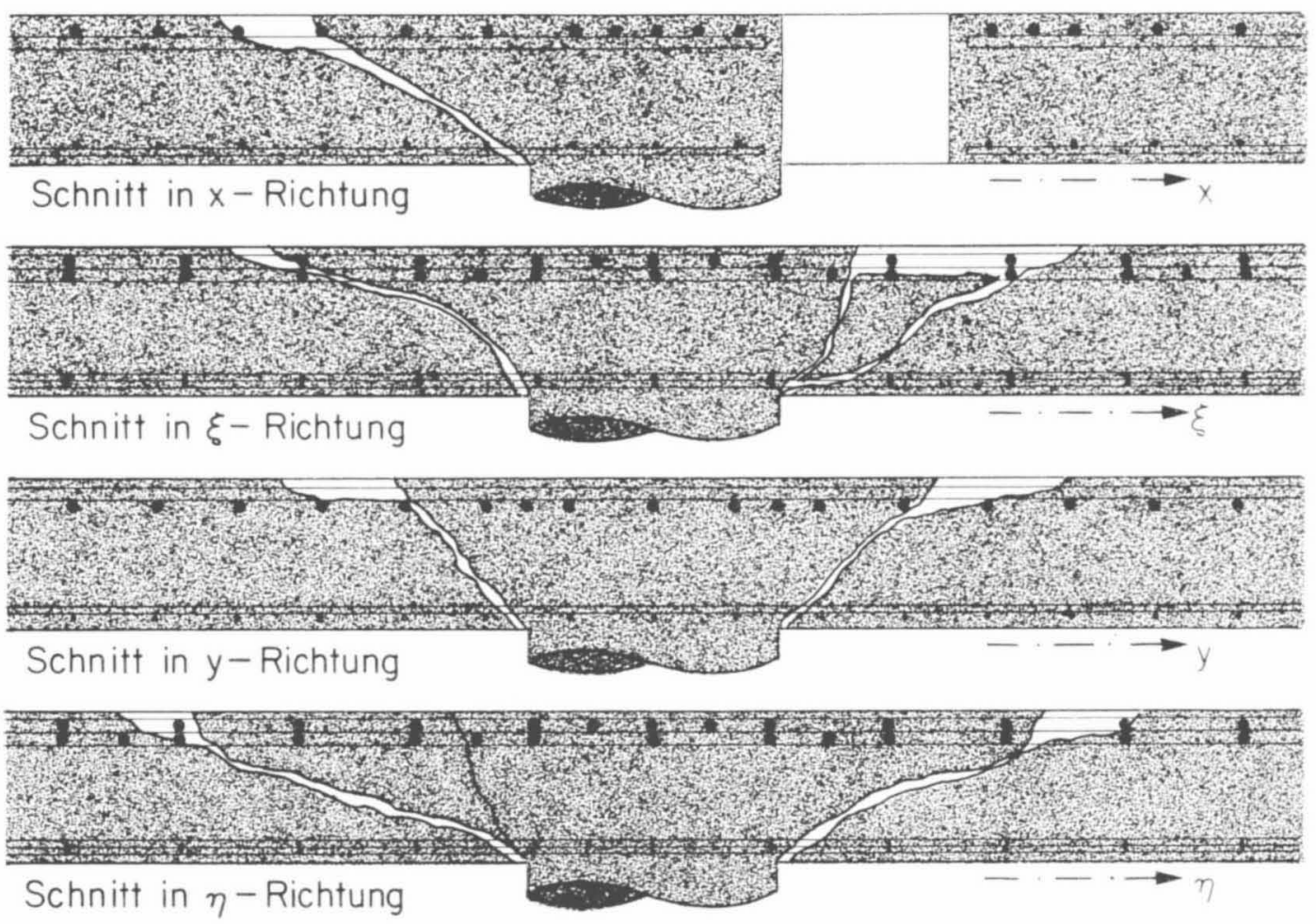

Bild 95: Profile der Bruchflächen, Platte P 16
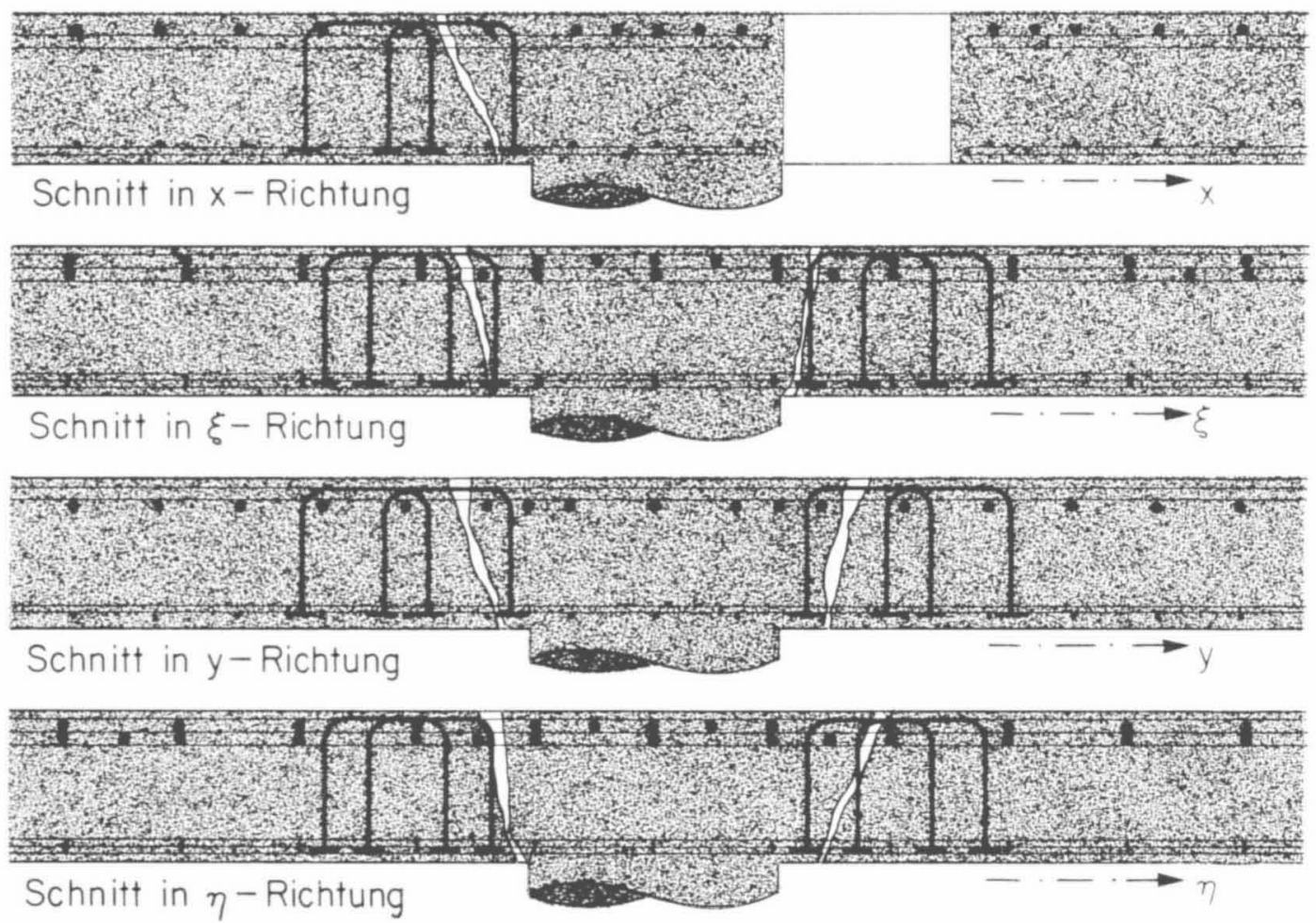

Bild 96: Profile der Bruchflächen, Platte P 17 

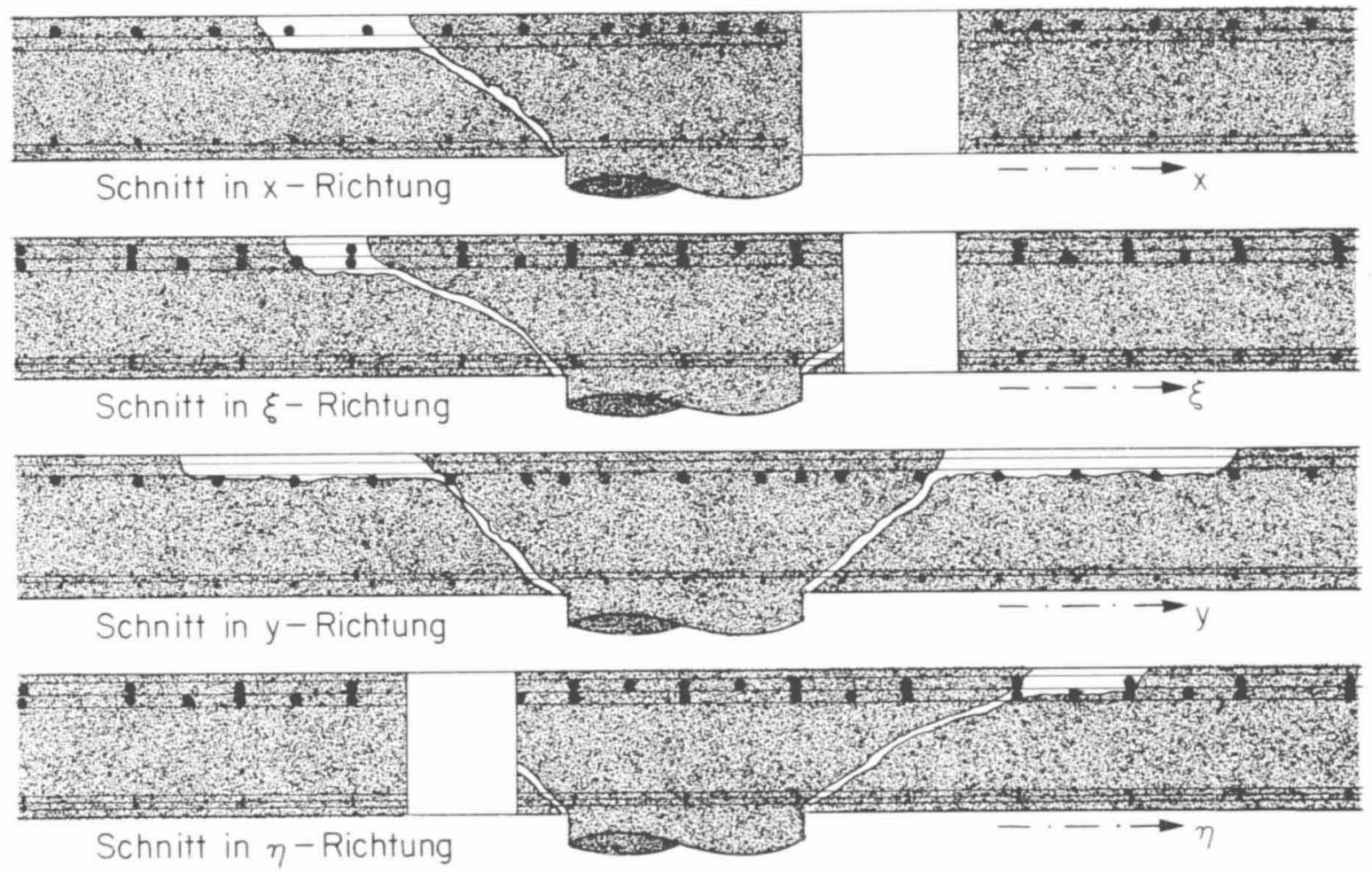

Bild 97: Profile der Bruchflächen, Platte P 18
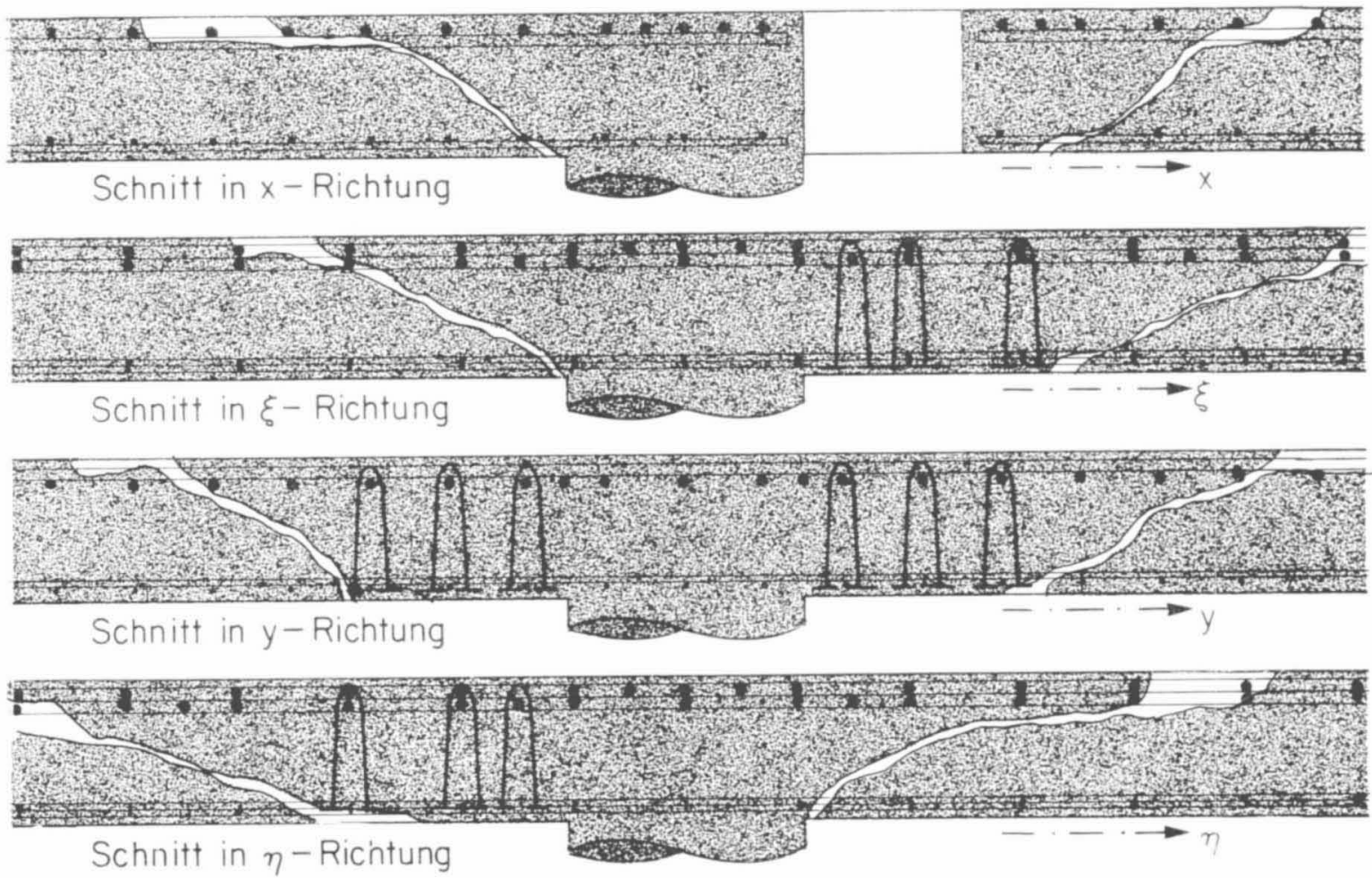

Bild 98: Profile der Bruchflächen, Platte P 19 

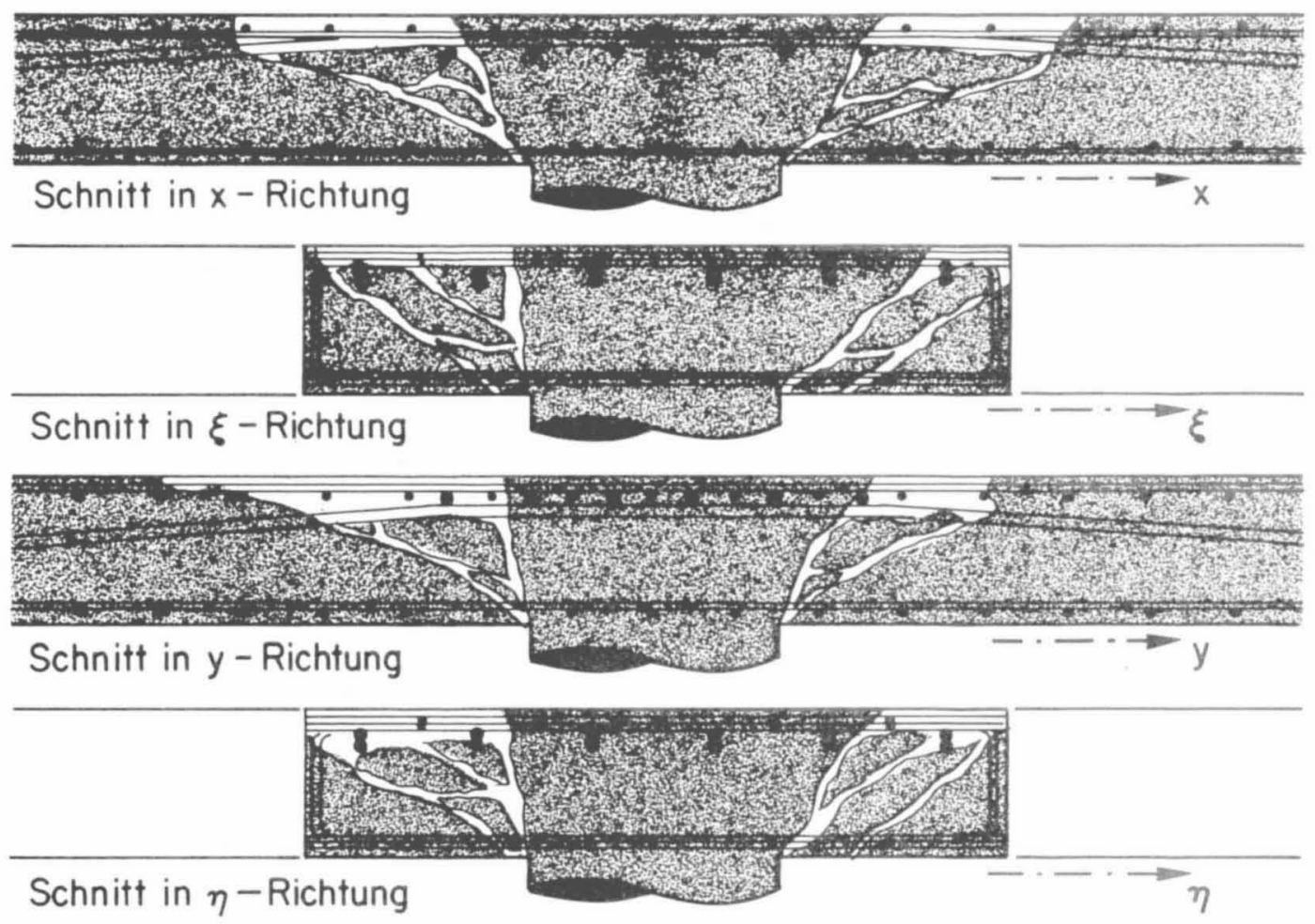

Bild 99: Profile der Bruchflächen, Platte P 20
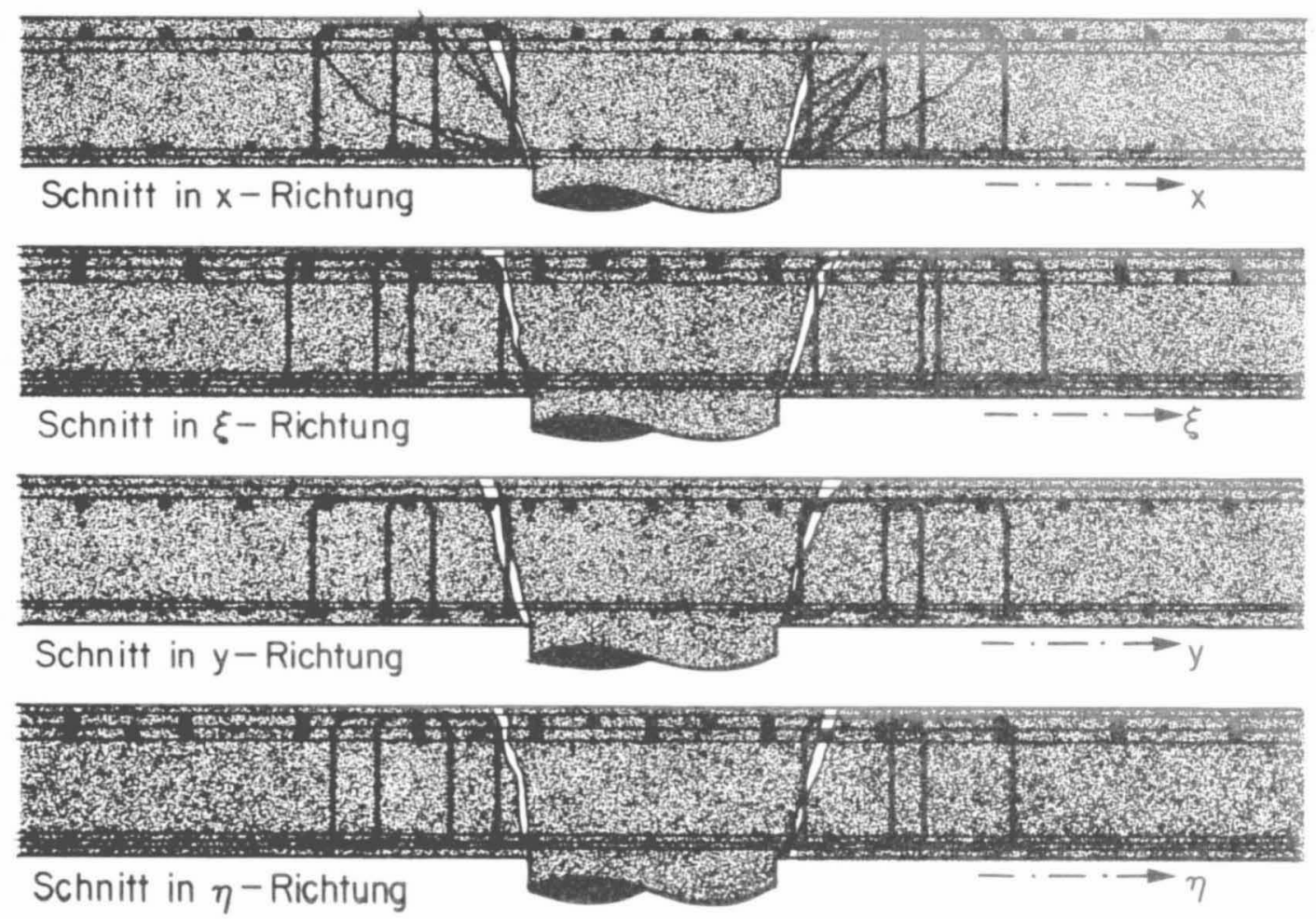

Bild 100: Profile der Bruchflächen, Platte P 22 


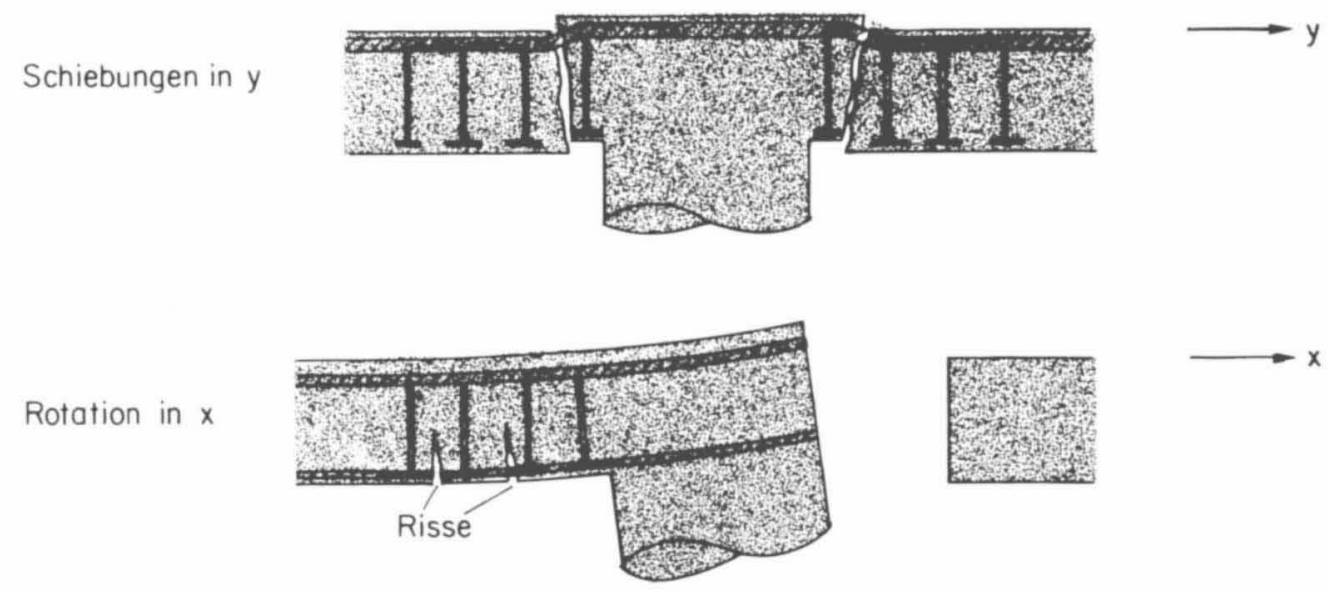

Bild 101: Bruchmechanismus, Platte P 17

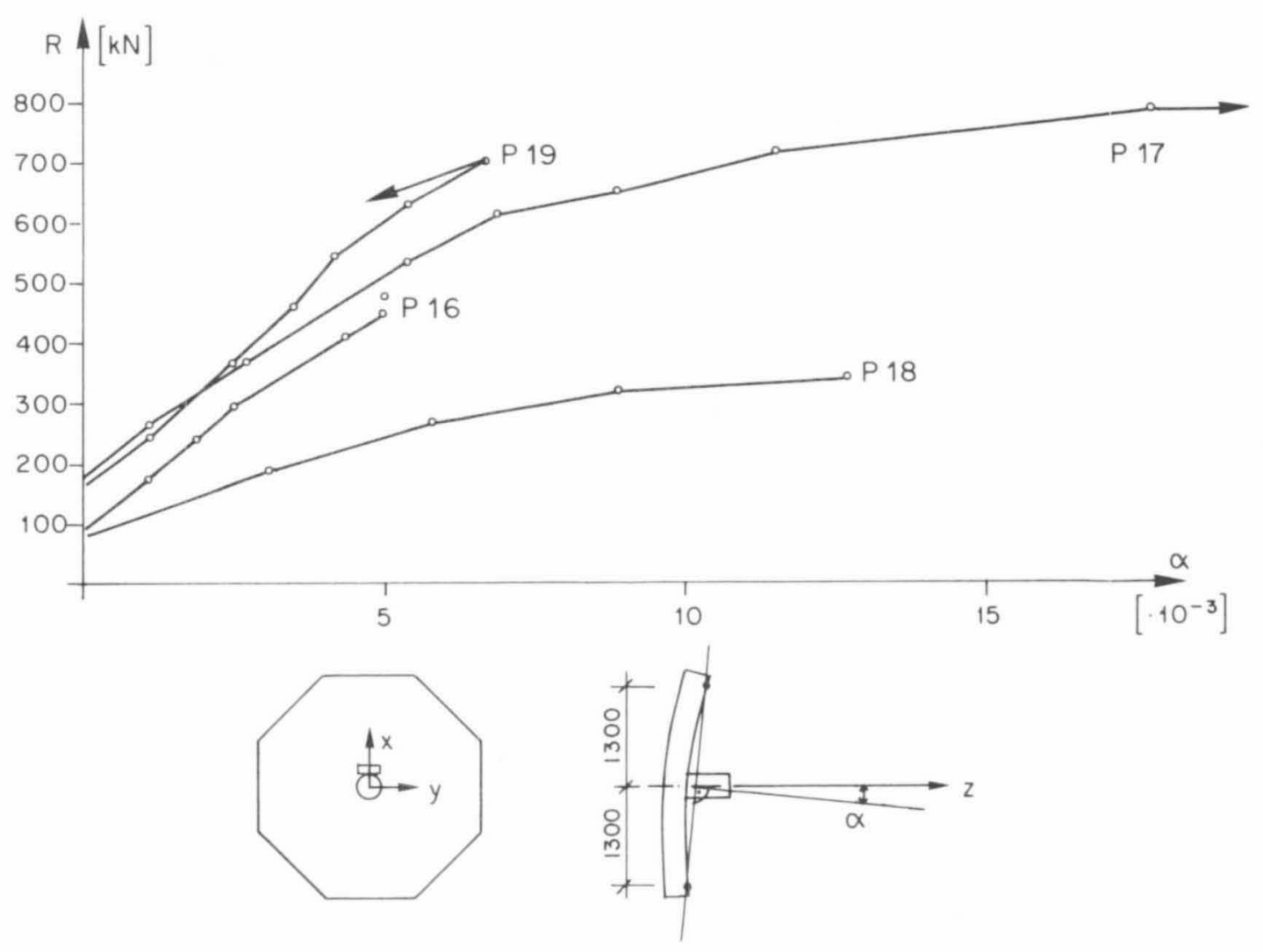

Bild 102: Verlauf der Verdrehung Platte - Stütze 


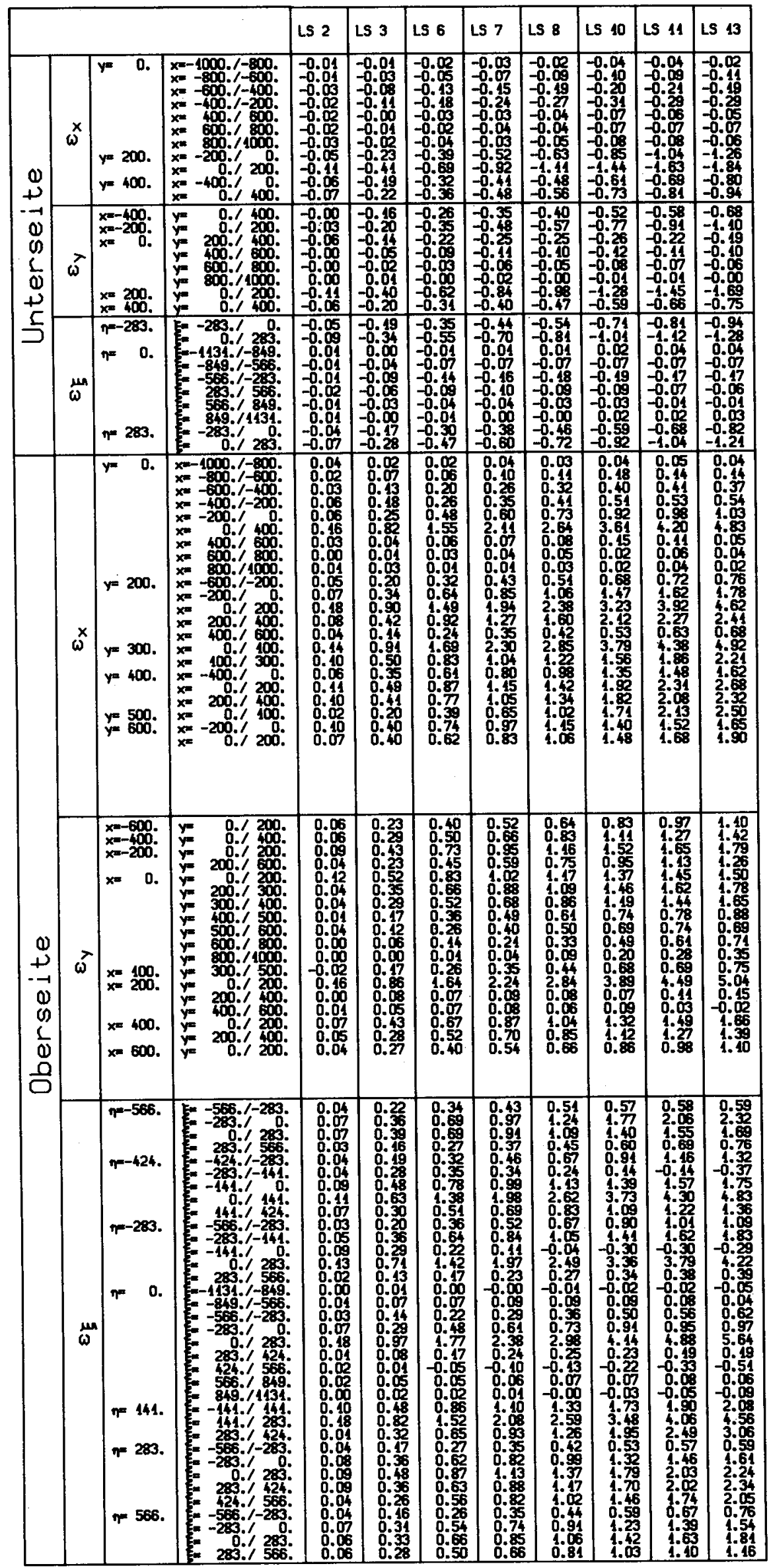

Tab, Al: Ausgeglichene mittlere Dehnungen in \%o, Platte $P 16$ 


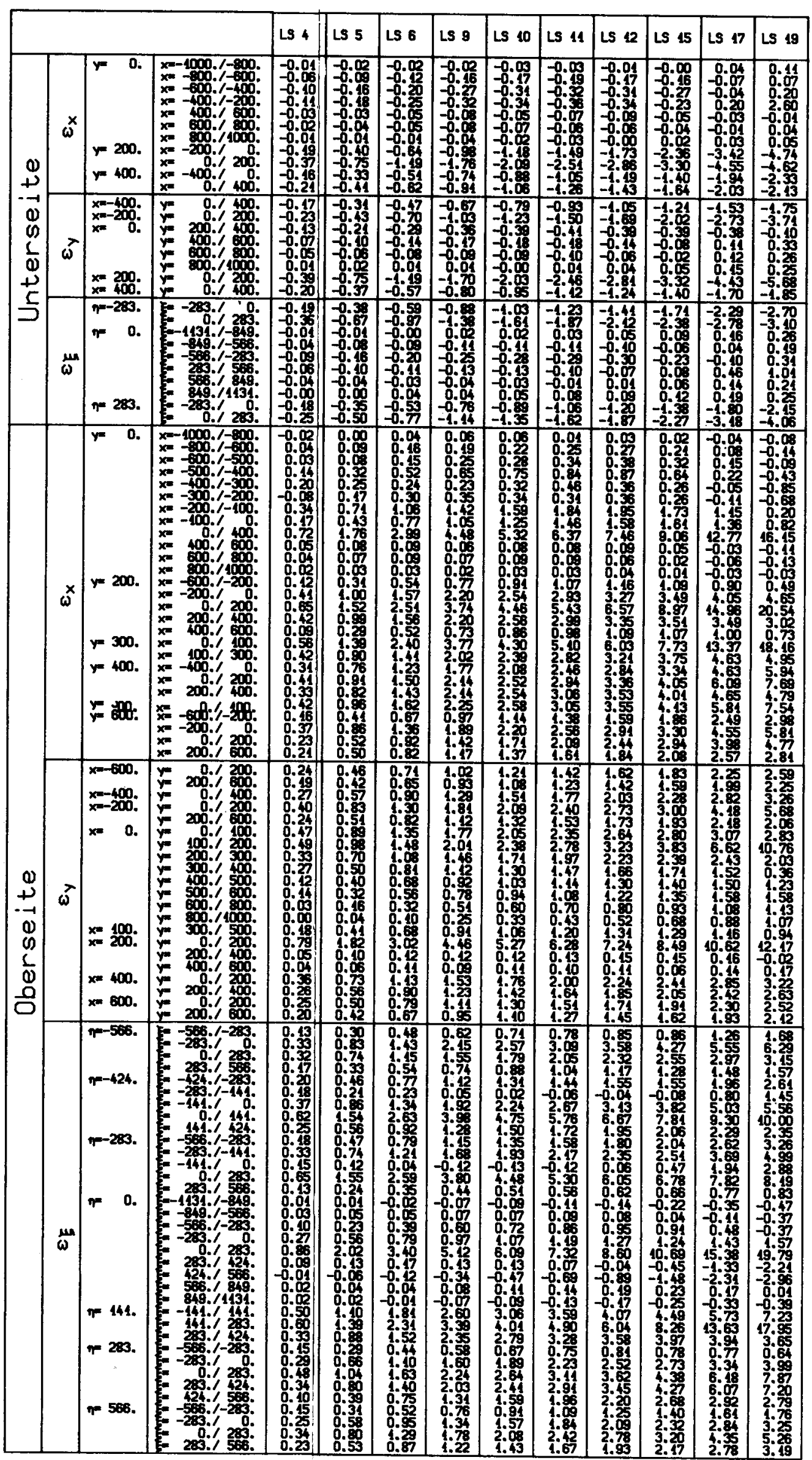

Tab. A2: Ausgeglichene mittlere Dehnungen in \%o, Platte P 17 


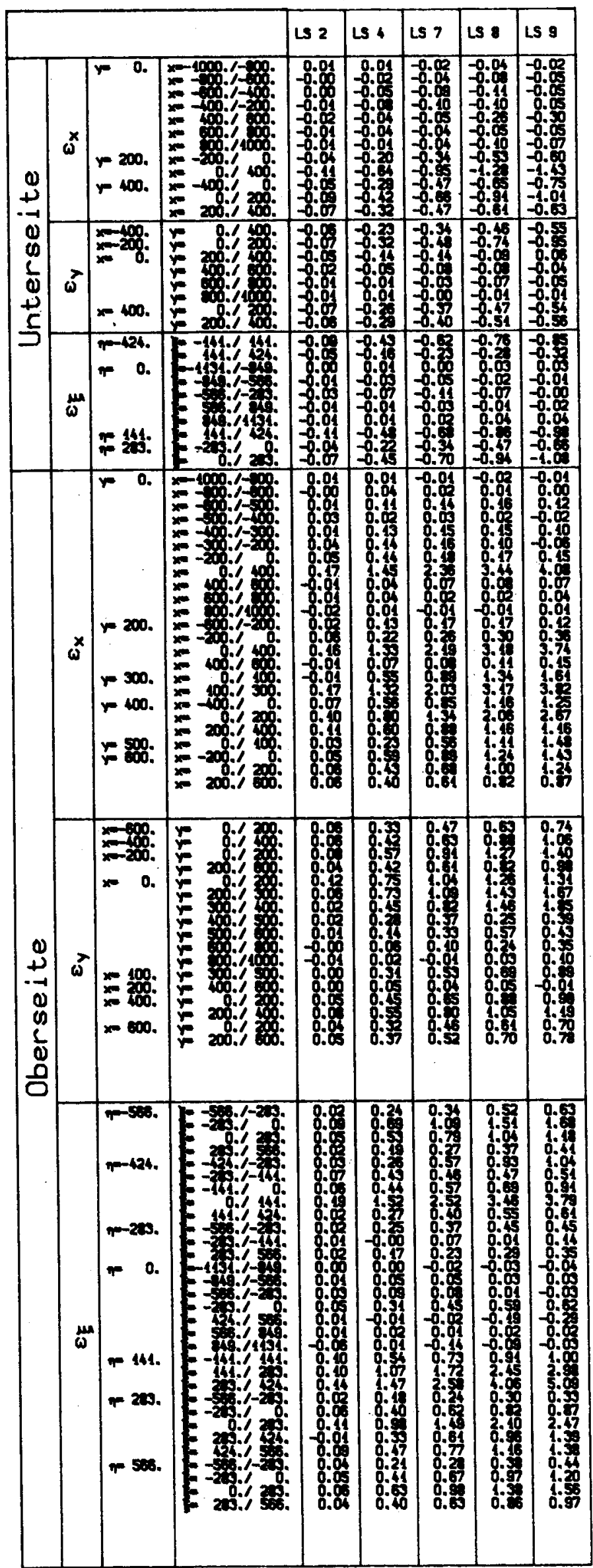

Tab. A3: Ausgeglichene mittlere Dehnungen in \%o, Platte $P 18$ 


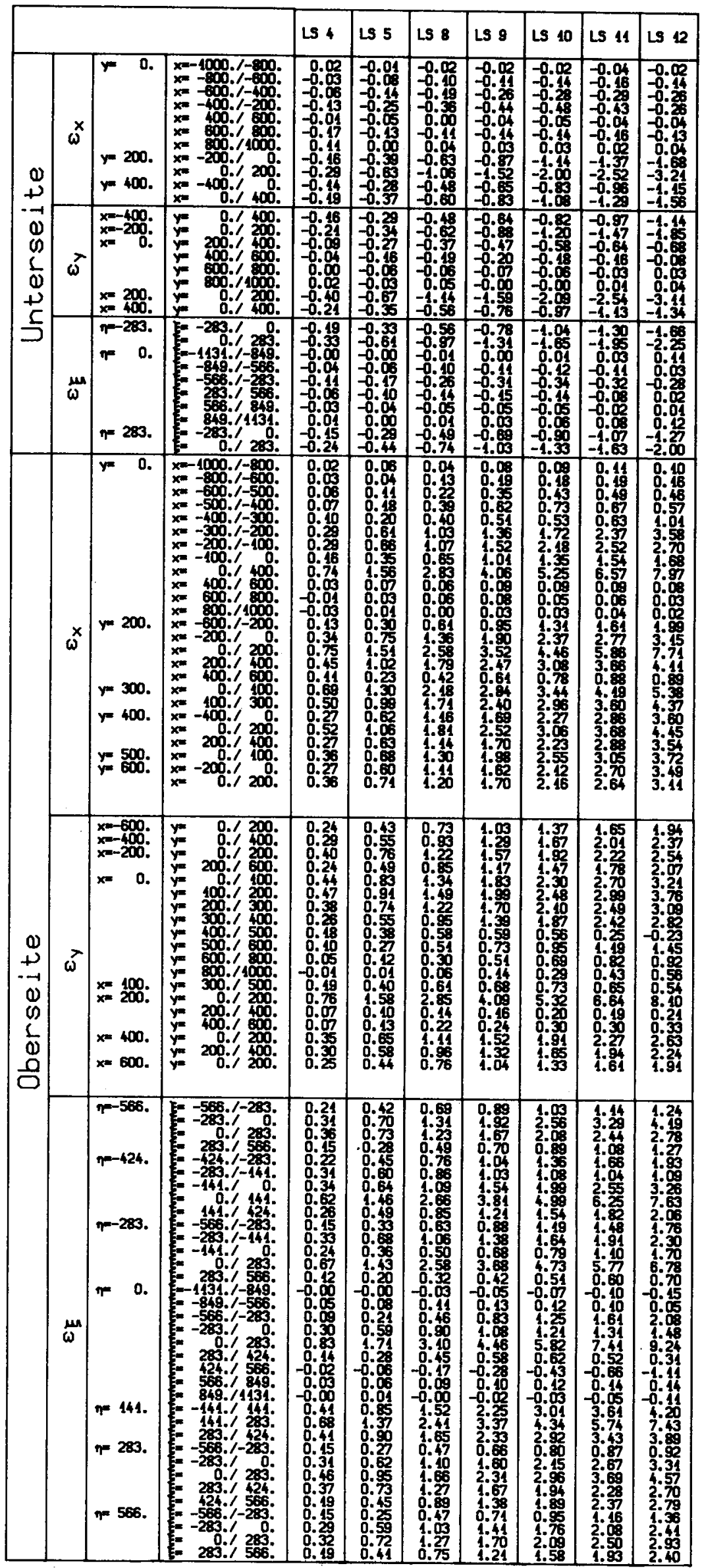

Tab. A4: Ausgeglichene mittlere Dehnungen in \%o, Platte P 19 


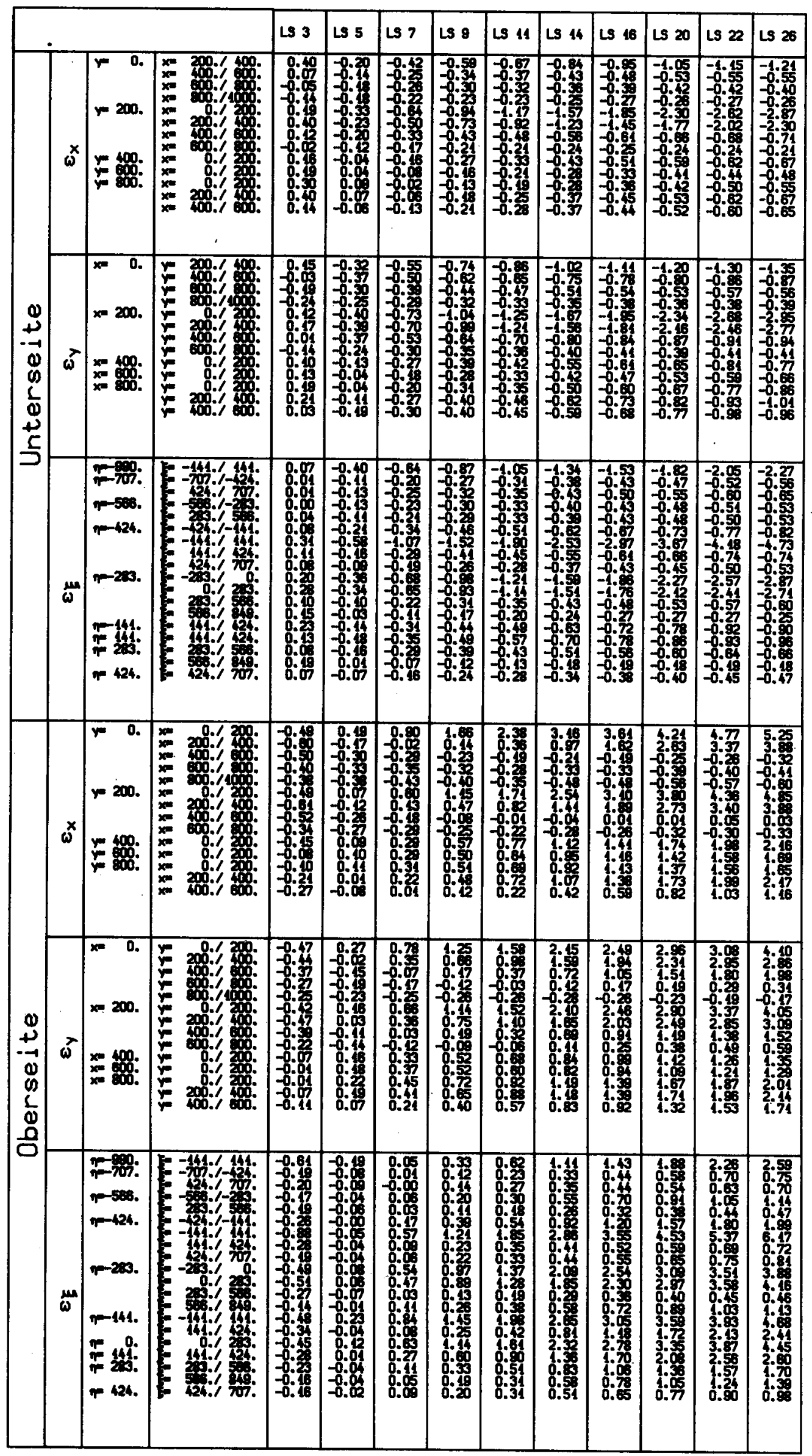

Tab. A5: Ausgeglichene mittlere Dehnungen in \%o, Platte P 20 


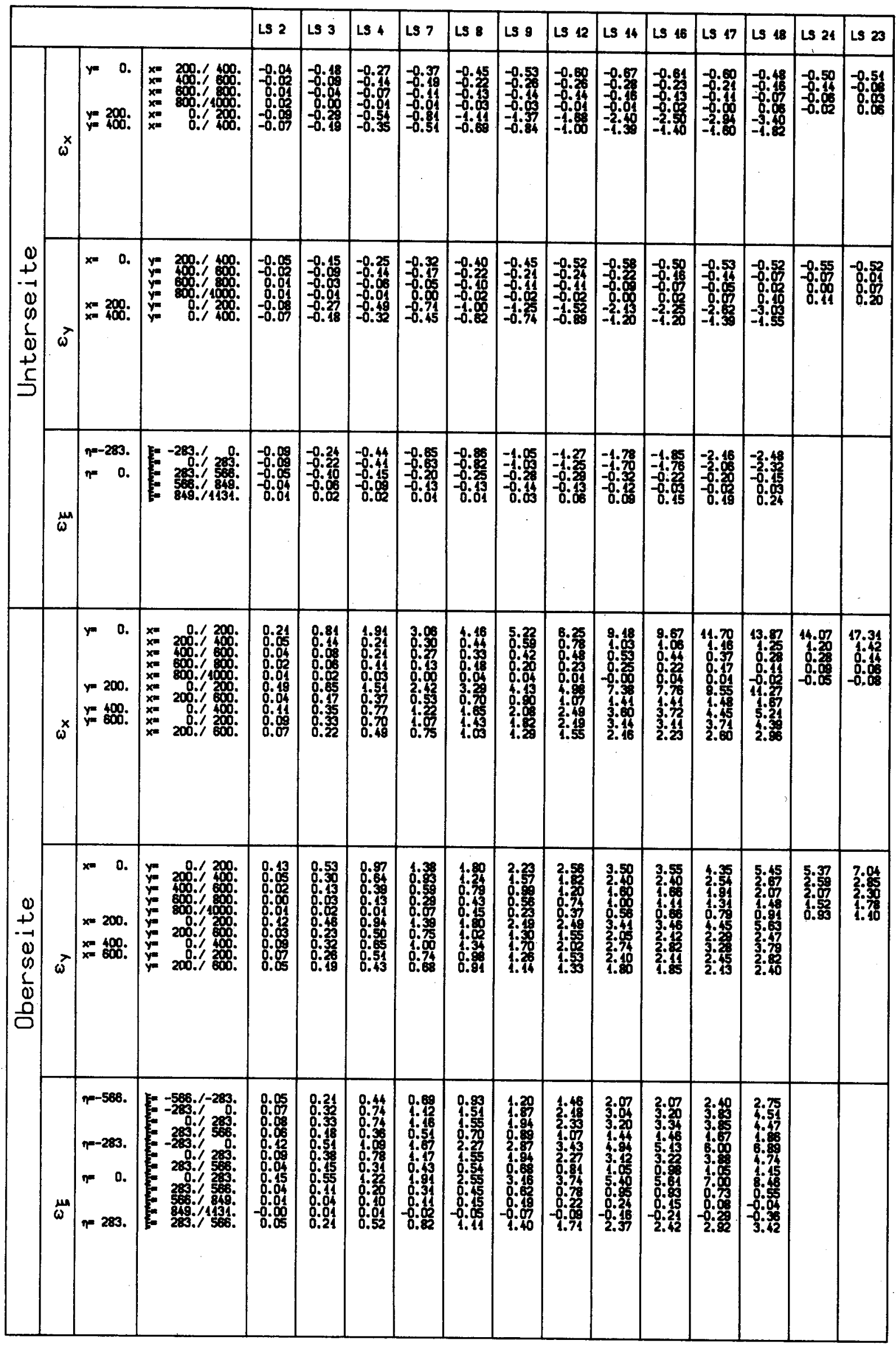

Tab. A6: Ausgegllchene mittlere Dehnungen in $\% 0$, Platte P 22 